\title{
Ghrelin-axis function in the context of Adult Hippocampal Neurogenesis
}

Hornsby, Amanda K. E.

How to cite:

Hornsby, Amanda K. E. (2018) Ghrelin-axis function in the context of Adult Hippocampal Neurogenesis. Doctoral thesis, Swansea University.

http://cronfa.swan.ac.uk/Record/cronfa50587

Use policy:

This item is brought to you by Swansea University. Any person downloading material is agreeing to abide by the terms of the repository licence: copies of full text items may be used or reproduced in any format or medium, without prior permission for personal research or study, educational or non-commercial purposes only. The copyright for any work remains with the original author unless otherwise specified. The full-text must not be sold in any format or medium without the formal permission of the copyright holder. Permission for multiple reproductions should be obtained from the original author.

Authors are personally responsible for adhering to copyright and publisher restrictions when uploading content to the repository.

Please link to the metadata record in the Swansea University repository, Cronfa (link given in the citation reference above.)

http://www.swansea.ac.uk/library/researchsupport/ris-support/ 


\title{
Ghrelin-axis function in the context of Adult Hippocampal Neurogenesis.
}

\author{
Amanda Kalliopi Emma Hornsby MSc
}

Supervisors:

Dr Jeffrey S. Davies

Prof. Catherine A. Thornton

Submitted to Swansea University in fulfilment of the requirements for the Degree of Doctor of Philosophy

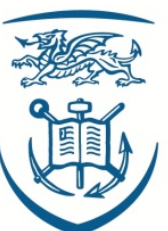

Swansea University

Prifysgol Abertawe

2018 


\begin{abstract}
The dentate gyrus (DG) is a neurogenic niche in the adult mammalian brain where new neurones are formed from neural stem/progenitor cells (NSPCs) throughout life. These adult born neurones play a key role in learning and memory, however, this process is not fully understood.

Calorie restriction (CR) has been shown to modulate the DG and improve cognition albeit via unknown mechanisms. The gut hormone ghrelin, which is acylated by Ghrelin-O-acyl transferase (GOAT), is elevated during $C R$ and travels via the blood to the brain where it binds to its receptor, GHSR, in several sites, including the hippocampus. We show that acyl-ghrelin significantly increases adult hippocampal neurogenesis (AHN) in the DG.
\end{abstract}

Immunofluorescent analysis of adult male GHSR-eGFP mice showed no GHSR expression in Sox $2^{+}, \mathrm{Nestin}^{+}$or $\mathrm{Ki}^{+} 7^{+} \mathrm{NSPCs}$ in the DG, but confirmed high expression on mature DG NeuN ${ }^{+}$cells, suggesting a non-cell autonomous mechanism of action. Following this, we show that CR enhances AHN in wild-type but not GHSR-KO mice, demonstrating that CR induces AHN in a GHSR-dependant manner.

To determine whether un-acylated ghrelin treatment negatively affects AHN, wild-type and GOAT-KO mice, which have an absence of circulating acyl-ghrelin, were treated with either vehicle or unacylatedghrelin. Wild-type mice treated with un-acylated ghrelin showed a significant reduction in proliferating $\left(\mathrm{Ki}^{+} 7^{+}\right)$cells and immature $\left(\mathrm{DCX}^{+}\right)$ neurones in the subgranular zone of the DG. Similarly, neurogenesis was impaired in GOAT-KO mice.

Lastly, to determine whether our rodent work was relevant to humans, circulating acyl-ghrelin was quantified in patients diagnosed with Parkinson's disease dementia (PDD). Notably, fasted and post-prandial plasma acyl-ghrelin levels were significantly reduced in PDD patients relative to healthy age-matched controls.

In summary, the results from this thesis suggest that acyl-ghrelin may be a biomarker for dementia, shown in PDD, and that elevating circulating acyl-ghrelin or reducing unacylated-ghrelin represents a novel therapeutic approach for preventing the decline in AHN. 


\section{DECLARATION}

This work has not previously been accepted in substance for any degree and is not being concurrently submitted in candidature for any degree.

Signed

(candidate)

Date

\section{STATEMENT 1}

This thesis is the result of my own investigations, except where otherwise stated. Where correction services have been used, the extent and nature of the correction is clearly marked in a footnote(s).

Other sources are acknowledged by footnotes giving explicit references. A bibliography is appended.

Signed (candidate)

Date

\section{STATEMENT 2}

I hereby give consent for my thesis, if accepted, to be available for photocopying and for inter-library loans after expiry of a bar on access approved by the Swansea University.

Signed (candidate)

Date 


\section{ACKNOWLEDGEMENTS}

Firstly, I would like to thank my supervisor Dr Jeff Davies for giving me the opportunity to be a part of his lab, and encouraging me to take on this project. Thank you for your guidance and patience. Who knew some voluntary work would turn into all of this.

Cerys, Gemma, Nick and Lynwen, your encouragement and support have meant the world to me, and it has helped me so much on this mental minefield of a journey. The numerous cups of tea, Jaffa cakes, custard creams and power chats have got me through it all. You guys are awesome and I'll be forever grateful.

I would like to say thank you to my parents for never saying that I can't do anything I set my mind to. I believe I get my resilience and determination from you both. To my brother William, you have always been my inspiration. Your belief in me and our chats these last few months have been invaluable, love you dude.

Dave. Thank you for putting up with me on this emotional and sometimes ugly rollercoaster, I know it hasn't been easy on you. I promise normal life can resume now, and I may even do the dishes. 


\section{CONTENTS}

Chapter 1 General Introduction ..................................................................1

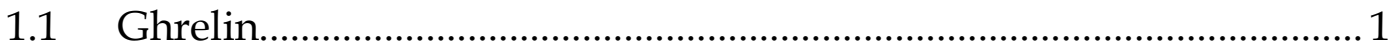

1.1.1 Acylation of Ghrelin. ........................................................................ 1

1.1.2 Role in energy homeostasis and orexigenesis................................

1.1.3 Lessons from Ghrelin $\mathrm{KO}$ mice ……………………………………....

1.2 The Role of G-Protein Coupled Receptors (GPCR's) ……………........ 7

1.2.1 Ghrelin Receptor …………………………............................... 8

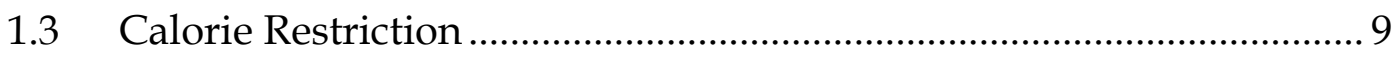

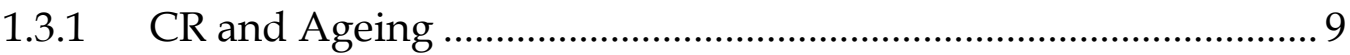

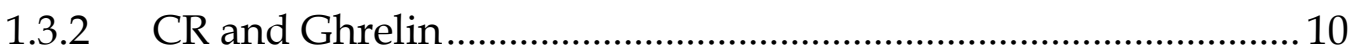

$1.4 \quad$ Adult Neurogenesis ...................................................................... 11

1.4.1 Adult Hippocampal Neurogenesis................................................... 13

1.4.2 Regulation of AHN …………………………………………...... 16

1.4.3 Role of AHN in Hippocampal-dependent Learning and Memory 18

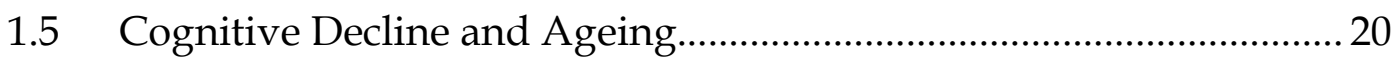

1.5.1 Parkinson's disease, AHN and Cognitive Decline........................ 21

1.5.2 Parkinson's disease and Ghrelin..................................................... 23

1.6 Aims and Hypothesis..................................................................... 23

Chapter 2 General Methods...................................................................... 26

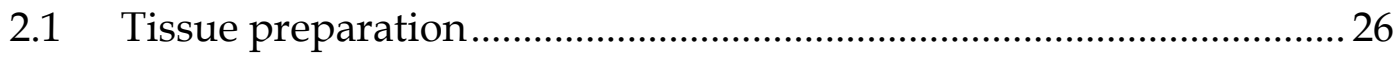

2.2 Tissue Sectioning ........................................................................... 26

2.3 Immunohistochemical staining protocols............................................. 27 
2.3.1 Standard protocol of immunofluorescent staining for multiple targets. 27

2.3.2 Standard protocol of immunofluorescent staining for multiple targets requiring antigen retrieval (AR), with citrate buffer. 28

2.3.3 Standard protocol of immunofluorescent staining for the target Bromo-deoxyuridine (BrdU).

2.3.4 Standard method of immunohistochemical staining using Nickel-enhanced Di-amino benezidine (Ni-DAB). .29

2.3.5 Standard method of immunohistochemical staining using Ni$\mathrm{DAB}$ with targets that require AR with Citrate Buffer. 30

\section{Chapter 3 Phenotypic Characterisation of the ghrelin receptor}

(GHS-R) in the adult mouse brain.................................................... 31

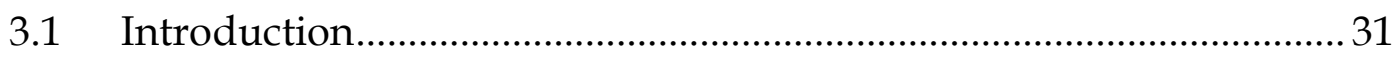

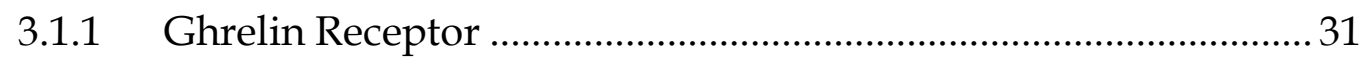

3.1.2 GHSR-eGFP transgenic mouse model ..................................... 32

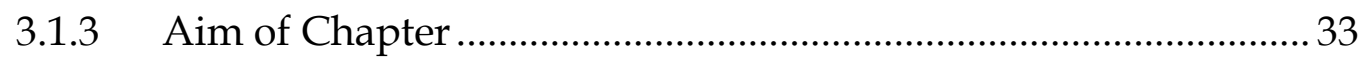

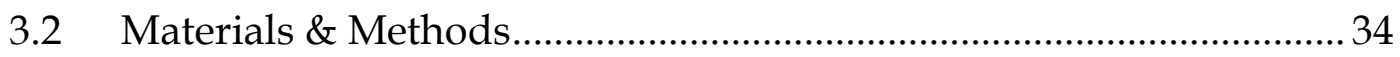

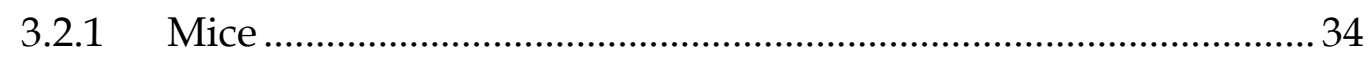

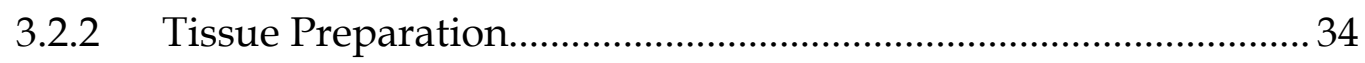

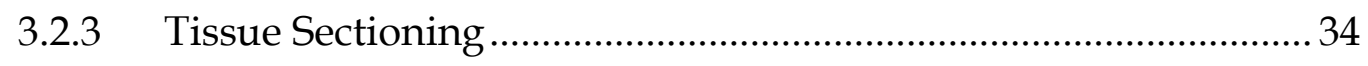

3.2.4 Immunofluorescent staining of Type I NSPC's, proliferating cells and mature granule cells with GHSR-GFP. .35

3.2.5 Immunofluorescent staining of Type II NSPC's with GHSR-GFP. 35

3.2.6 Analysis of fluorescent immunoreactivity................................ 36

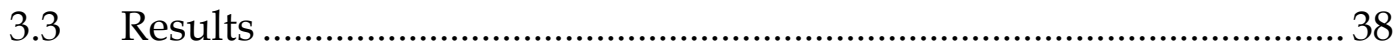


3.3.1 Phenotypic Characterisation of GHSR in the Dentate Gyrus of adult mice

\section{Chapter 4 The direct and indirect effects of ghrelin on adult}

\section{hippocampal plasticity} 45

4.1 Introduction .45

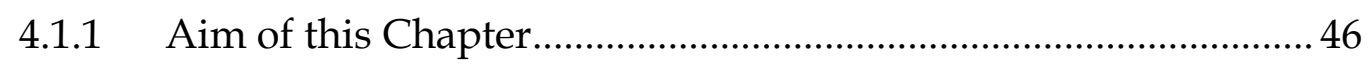

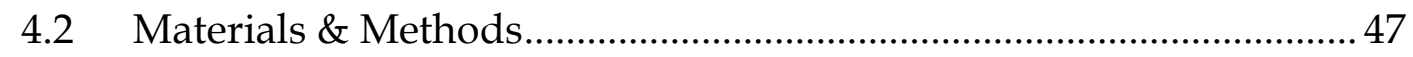

4.2.1 Mice and Experiments ........................................................... 47

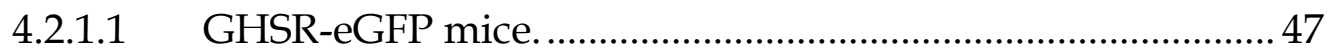

4.2.1.2 GHSR knock out mice...................................................... 47

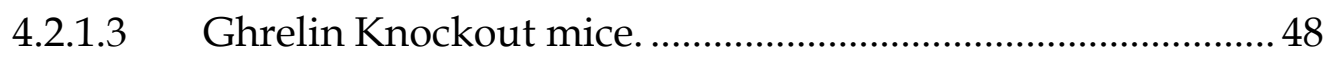

4.2.1.4 Behavioural Testing: Contextual Fear Conditioning ............ 49

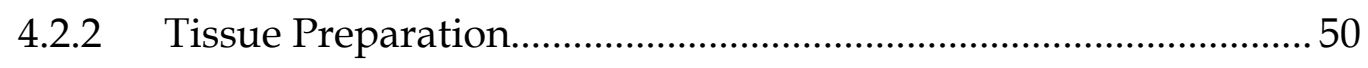

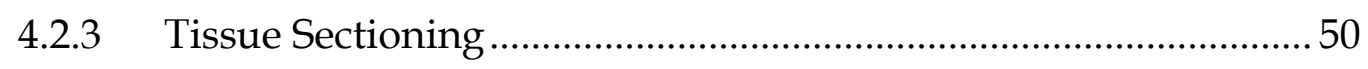

4.2.4 Immunoflurescent staining of immediate early gene (IEG) activation in GHSR-eGFP mice. ............................................................. 50

4.2.5 Immunofluorescent staining of proliferating NSPC's, in GHSReGFP mice. .54

4.2.6 Immunofluorescent staining of new-born neuronal cells (BrdUNeuN), in GHSR -/- mice and WT littermates.

4.2.7 Immunohistochemical staining for Type II NSPC's (Sox2+) using Nickel enhanced DAB (di-aminobenzidine) (Ni-DAB) ............................ 55

4.2.8 Immunohistochemical staining for immature neuronal cells (DCX) using Ni-DAB. .55

4.2.9 Immunohistochemical staining for new-born cells (BrdU) using Ni-DAB. 55 
4.2.10 Immunofluorescent staining of new-born neuronal cells (BrdU/NeuN), and new-born neuronally active cells (BrdU/c-Fos), in WT and Ghrelin KO mice. .57

4.2.11 Immunofluorescent staining of new-born neuronal cells (BrdU$\mathrm{NeuN}$ ), in WT and Ghrelin KO mice. .58

4.2.12 Analysis of fluorescent immunoreactivity. .58

4.2.13 Analysis of Ni-DAB immunoreactivity ..................................... 58

4.2.14 Data and Statistical Analysis 59

4.2.14.1 IEG activation in GHSR-eGFP mice....................................59

4.2.14.2 Cell proliferation in the DG of GHSR-eGFP mice.................59

4.2.14.3 Effect of CR on AHN in the adult DG of GHSR KO mice. .. 59

4.2.14.4 Effect of genotype and CR on Type II NSPC number in WT and GHSR KO mice. 60

4.2.14.5 Effect of Ghrelin KO on basal neurogenesis levels. 60

4.2.14.6 Effect of CR on new-born neuronal cell activation in WT and Ghrelin-KO. 60

4.2.14.7 Effect of age on new-born neurone and cell activation. 61

4.3 Results 62

4.3.1 The effects of acutely elevating AG levels directly and indirectly in the mouse brain.

4.3.1.1 CR and AG treatment enhance the expression of the IEG Egr-1 in the DG.

4.3.1.2 CR and AG had no effect on cell proliferation or the type of NSPC's produced. .66

4.3.2 Acute CR enhances $\mathrm{AHN}$ and context dependant fear memory in a GHSR-dependant manner. .68

4.3.2.1 Acute CR has no effect on the Type II NSPC pool in the DG of WT and GHSR-KO mice.

4.3.3 The absence of circulating ghrelin has no effect on the numbers of type II NSPC's, proliferating cells, immature and new-born neuronal cells in the DG. 
4.3.3.1 Ghrelin $\mathrm{KO}$ mice show normal rate of new adult-born neurone activation following CR......................................................... 74

4.3.3.2 The effect of age on new neuronal cell survival and activation. .77

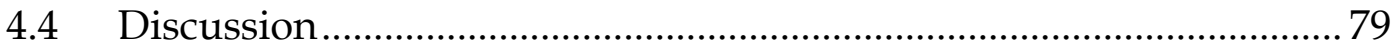

Chapter 5 The effect of UAG and AG on AHN ............................84

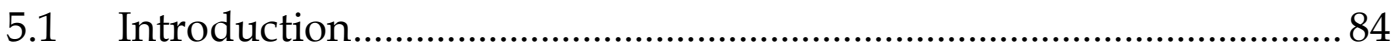

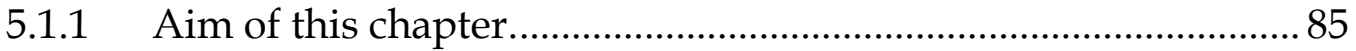

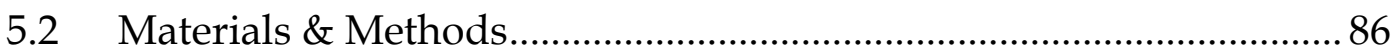

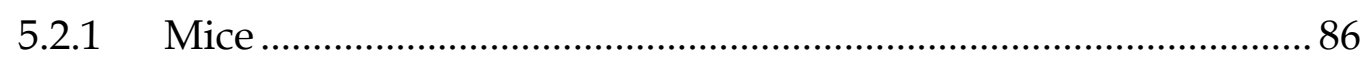

5.2.1.1 UAG treatment in WT and GOAT KO mice .........................86

5.2.1.2 AG treatment in WT and GOAT KO mice ........................... 86

5.2.1.3 UAG treatment in WT mice................................................. 87

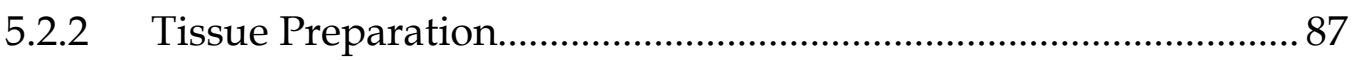

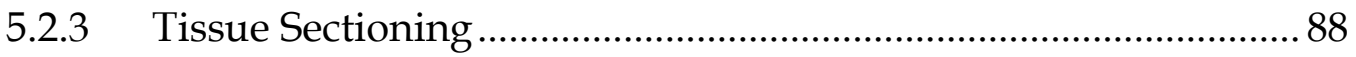

5.2.4 Immunofluorescent staining of recently divided immature neuronal cells (BrdU-DCX) in 6 month old WT and GOAT KO mice..... 88

5.2.5 Immunofluorescent staining of newborn mature neuronal cells (BrdU-NeuN) in 12 week old WT and GOAT KO mice. 89

5.2.6 Immunohistochemical staining of immature neurones (DCX) in WT and GOAT KO mice, using Ni-DAB.

5.2.7 Immunohisotchemical staining of proliferating (Ki67) cells, type II NSPC's (Sox2) and active (Egr-1) neurones, in WT and GOAT KO mice, using Ni-DAB

5.2.8 Immunohistochemical staining of active (c-Fos) neurones in WT and GOAT KO mice, using Ni-DAB.

5.2.9 Impregnation of WT and GOAT KO mouse brains with Golgi Cox solution. .92 
5.2.10 Sectioning and processing of Golgi-Cox impregnated brain tissue. 92

5.2.11 Analysis of fluorescent immunoreactivity. .............................. 93

5.2.12 Analysis of Ni-DAB immunoreactivity.................................... 93

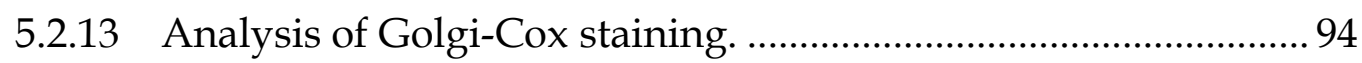

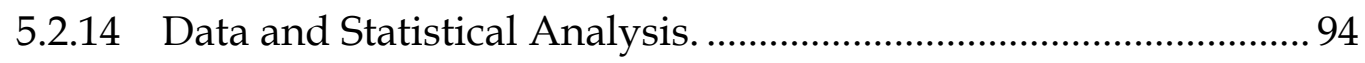

5.2.14.1 Effect of UAG treatment on AHN in WT and GOAT KO mice. 94

5.2.14.2 Effect of UAG treatment on Type II NSPC pool in WT and

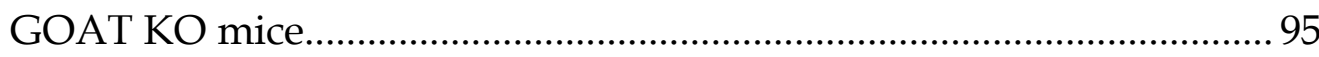

5.2.14.3 Effect on AHN and Type II NSPCs in second GOAT KO

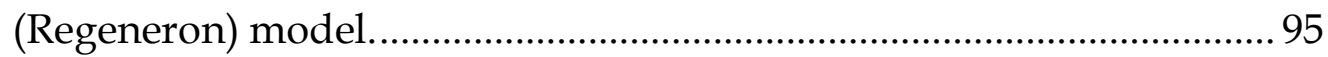

5.2.14.4 Effect on IEG expression in second GOAT KO model......... 95

5.2.14.5 Effect of genotype on dendritic spine formation.................. 96

5.2.14.6 Effect of short-term AG treatment on AHN in WT and

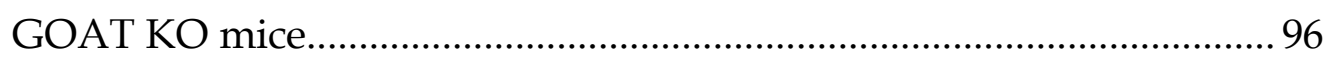

5.2.14.7 Effect of UAG treatment on AHN in WT C57BL/6 mice..... 96

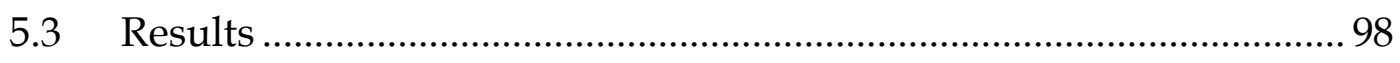

5.3.1 UAG treatment of adult WT and GOAT KO mice leads to a reduction of $\mathrm{AHN}$. .98

5.3.1.1 Genetic knockout of GOAT or UAG treatment reduced the number of new-born immature neurones and new-born non-neuronal cells in the SGZ of the DG.

5.3.2 GOAT-KO mice show no significant effect on the type II NSPC pool compared to WT controls. 101

5.3.3 A second GOAT KO model shows deficits in AHN, but not type II NSPC number. 102

5.3.4 Immediate Early Gene (IEG) expression was unaffected in the DG following 7 day acyl-ghrelin treatment in WT and GOAT KO mice. 104 
5.3.5 Genotype has no overall effect on dendritic spine formation. .105

5.3.6 Period of time post-acyl-ghrelin treatment significantly affects rates of new neuronal cell production. 107

5.3.7 UAG treatment of WT mice does not affect AHN. 109

5.4 Discussion

Chapter 6 The Effect of Calorie Restriction on circulating AGUAG in Healthy, Parkinson's Disease and Parkinson's Disease Dementia patients 116

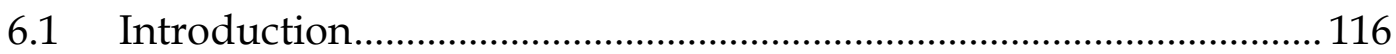

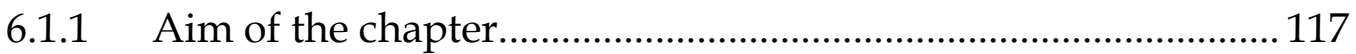

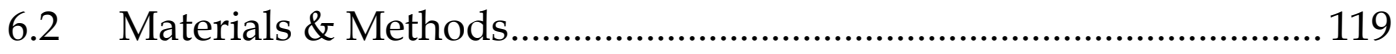

6.2.1 Study Design and Criteria ....................................................... 119

6.2.1.1 Participant criteria ............................................................. 119

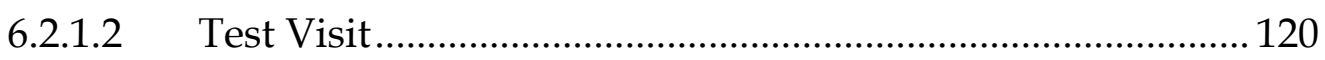

6.2.1.3 Blood collection and storage ............................................. 121

6.2.2 Total Ghrelin ELISA................................................................ 122

6.2.3 Multiple analyte analysis with Milliplex system...................... 123

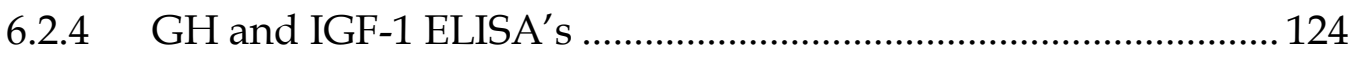

6.2.5 Data handling, Analysis and Statistics.................................... 125

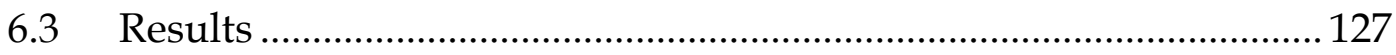

6.3.1 The effects of fasting and re-feeding on circulating analytes in the blood plasma of PD and PDD patients, and age-matched controls.127 6.3.2 Fasting and re-feeding effects on the AG:UAG....................... 131

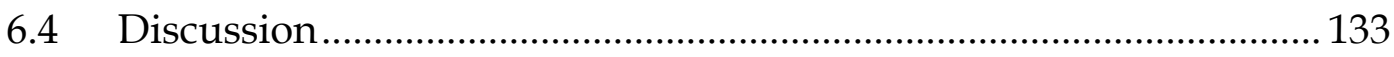

Chapter 7 General Discussion .................................................................138

7.1 Summary. 138 


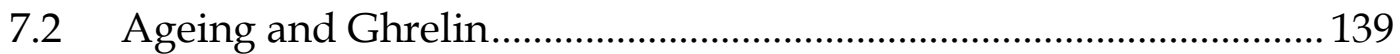

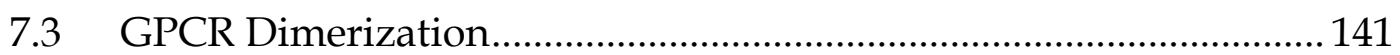

7.4 Ageing and Activation of Ghrelin....................................................... 142

7.5 Adult Hippocampal Neurogenesis in Ageing .................................... 143

7.6 Calorie Restriction and Ageing …………………….......................... 148

7.7 Drug development for Ghrelin....................................................... 149

Chapter 8 Appendix...................................................................................154

8.1 Buffer and Reagent Recipes ................................................................ 154

8.1.1 10x Phosphate Buffered Solution (PBS) (0.1M), pH 7.4 ............. 154

8.1.2 1x Phosphate Buffered Solution (PBS) (0.01M) ........................... 154

8.1.3 10x Tris Buffered Solution (TBS) pH 7.6 ………………………...154

8.1.4 1X Tris Buffered Solution (TBS) ……………………………....... 154

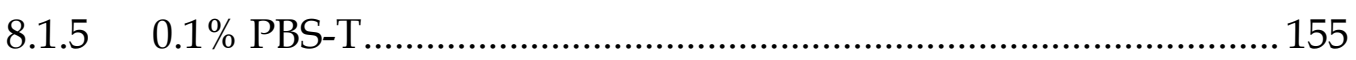

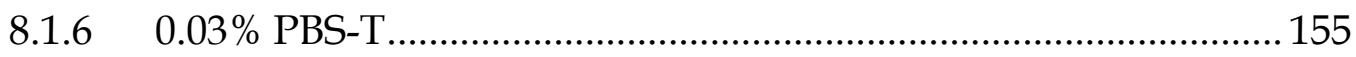

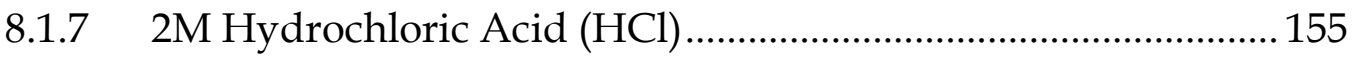

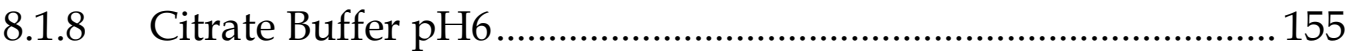

8.1.9 0.1M Sodium Acetate $\mathrm{pH} 6$ …………………………………........ 155

8.1.10 0.2M Sodium Acetate......................................................................... 156

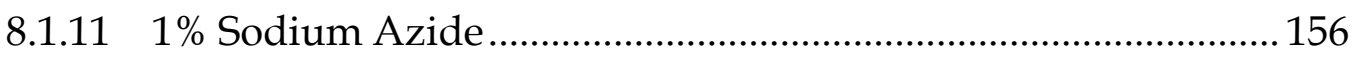

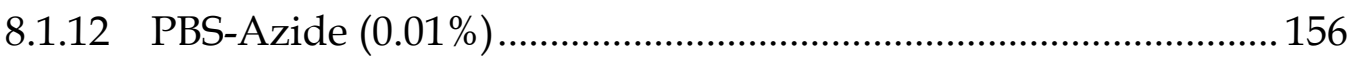

8.1.13 30\% Sucrose solution …………………………………………...... 156

8.1.14 5M Sodium Hydroxide ( $\mathrm{NaOH})$ solution ...................................... 156

8.1.15 4\% Paraformaldehyde (PFA) solution ......................................... 157

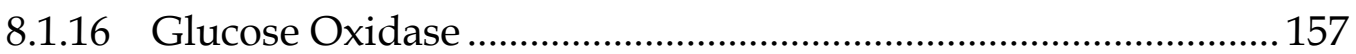

8.1.17 Nickel enhanced Diaminobenzidine (Ni-DAB) ........................... 157 


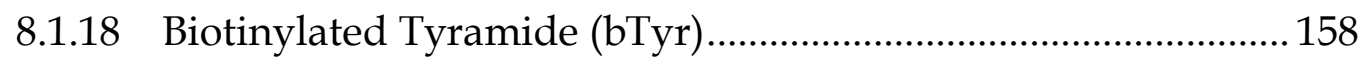

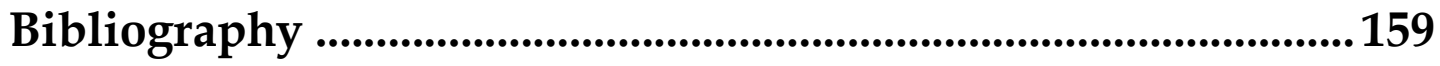




\section{TABLE OF FIGURES}

Figure 1.1 The structure of ghrelin and activation by acylation. ...................... 2

Figure 1.2 Topographic representation of GOAT_............................................. 3

Figure 1.3. Autoradiographs of the dentate gyrus depicting thymidine- $\mathrm{H}^{3}$ cells in rats.

Figure 1.4 Process of Neurogenesis in the Dentate Gyrus.

16

Figure 3.1 Representative images of GHSR-eGFP expression in the granule cell layer of the DG.

Figure 3.2. General GHSR-eGFP expression in the hippocampus and other areas associated with learning and memory.

Figure 3.3. GHSR is not expressed on Type I (Nestin+) or Type II (Sox2+) NSPC's.

Figure 3.4. Close association of GHSR-GFP+ cells with Type II SOX2+ NSCs suggests a possible paracrine function 40

Figure 3.5. Proliferating cells (Ki67) do not express GHSR in the DG. 41

Figure 3.6. Mature Granule cells (NeuN+) express GHSR in the DG. 41

Figure 4.1 Diagram depicting CFC behavioural test.

Figure 4.2 CR and AG enhance Egr-1 expression in areas of the brain important for learning and memory.

Figure 4.3 CR and AG enhance Egr-1 expression in areas of the brain important for learning and memory, continued

Figure 4.4 Acyl-ghrelin injection and/ or CR has no effect on cell proliferation in the DG

Figure 4.5. Schematic of GHSR KO mice experimental time-line. .68

Figure 4.6 A 2 week period of CR enhances AHN in the adult DG and improves context dependant fear memory. 70

Figure 4.7 Genotype has no effect on the number of type II NSPC's, and CR significantly increases type II NSPC number independent of GHSR. 71 Figure 4.8 Ghrelin $\mathrm{KO}$ mice, lacking in circulating AG and UAG, show no effects on basal rates of neurogenesis in the dentate gyrus. .73 
Figure 4.9 Mice lacking in circulating AG and UAG have no impairment in their response to $\mathrm{CR}$. .76

Figure 4.10 Age has no overall effect on the number of new adult-born neurones and new adult-born active cells in CR mice. .78

Figure 5.1 UAG treatment in WT and GOAT knockout mice impairs AHN. 100

Figure 5.2 UAG treatment in WT and GOAT knockout mice impairs AHN continued. 101

Figure 5.3 GOAT KO has no effect on type II NSPC number or structure of the DG. 102

Figure 5.4 Regeneron (Yang et al, (2008) GOAT knockout mice have reduction in proliferating cells and immature neurone number, type II NSPC pool unaffected. 103

Figure 5.5 IEG expression is un-affected by genotype or AG treatment in the Regeneron GOAT knockout mouse model. 105 Figure 5.6 GOAT knockout significantly decreased the number of Stubby dendritic spines. 106 Figure 5.77 days of AG treatment appears to reduce new neuronal cell number, however after 28 days new neuronal cell number increases. 108 Figure 5.8 UAG treatment in WT C57BL/ 6 mice has no effect on the number of new adult-born neurones. 110 Figure 6.1 Timelines and Area Under Curve (AUC) for circulating plasma analytes. 129

Figure 6.2 Timeline and AUC graphs continued........................................... 130

Figure 6.3 Timeline and AUC graphs continued...............................................131 Figure 6.4 The fasted and post-prandial AG:UAG ratio is significantly reduced in PDD patients, compared to age-matched controls. 132 Figure 7.1 Blood plasma levels of Total Ghrelin of WT and Ghrelin KO mice at different ages. 140 


\section{TABLE OF TABLES}

Table 3.1: List of Primary Antibodies used. ...................................................... 36

Table 3.2: List of Fluorescent Secondary Antibodies used............................... 36

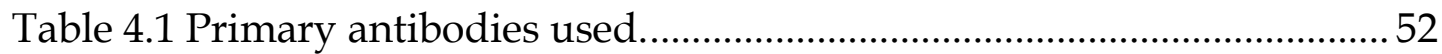

Table 4.2 Secondary antibodies and conjugated used. ................................... 53

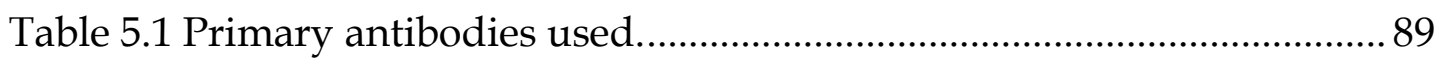

Table 5.2 Secondary antibodies and conjugates used..................................... 90

Table 6.1. Demographic information of study participants........................... 120 


\section{PUBLICATIONS}

Kent, B.A. et al., 2015. The orexigenic hormone acyl-ghrelin increases adult hippocampal neurogenesis and enhances pattern separation.

Psychoneuroendocrinology, 51, pp.431-439. Available at:

http://linkinghub.elsevier.com/retrieve/ pii/S0306453014003990.

Hornsby, A.K.E. et al., 2016. Short-term calorie restriction enhances adult hippocampal neurogenesis and remote fear memory in a Ghsr-dependent manner. Psychoneuroendocrinology, 63, pp.198-207. Available at:

http://linkinghub.elsevier.com/retrieve/ pii/S0306453015009269.

Hornsby, A.K.E. et al., 2018. Circulating unacylated-ghrelin impairs hippocampal neurogenesis and memory in mice and is altered in human Parkinson\&\#039;s disease dementia. bioRxiv. Available at: http:/ / biorxiv.org/content/ early/2018/02/05/259333.abstract. 


\section{ABBREVIATIONS}

AG

AGRP

AHN

APT1

AUC

BBB

BDNF

BLA

CingC

CNS

CR

DA

DG

ECL

GCL

GH

GHSR

GHSR1a

GHSR1b

GOAT

GPCR

I.C.V

I.P

IF

IHC

KO

LEAP2

LEnt

LTP
Acyl Ghrelin

Agouti-related protein

Adult hippocampal neurogenesis

Acyl protein thioesterase

Area under curve

Blood-brain barrier

Brain derived neurotropic factor

Basal Lateral Amygdala

Cingulate Cortex

Central nervous system

Calorie Restriction

Dopamine/Dopaminergic

Dentate gyrus

Extracellular loop

Granule cell layer

Growth Hormone

Growth Hormone Secretagogue Receptor

Growth Hormone Secretagogue Receptor 1a

Growth Hormone Secretagogue Receptor $1 \mathrm{~b}$

Ghrelin-O-acyl transferase

G-protein Coupled Receptor

Intracerebroventricular

Intraperitoneal

Immunofluorescence

Immunohistochemistry

Knockout

Liver-expressed antimicrobial peptide 2

Lateral Entorhinal Cortex

Long-term potentiation 
MBOAT4

MWM

NOR

NPY

OB

PD

PD+MCI

PDD

POMC

RGL

SGZ

SNpc

SVZ

TG

TM

UAG

WT
Membrane-bound O-acyltransferase

$$
\text { Morris Water Maze }
$$

Novel object recognition task

Neuropeptide $Y$

Olfactory bulb

Parkinson's disease

Parkinson's disease with mild cognitive impairment

Parkinson's disease with dementia

Pro-opiomelanocortin

Radial glial-like cells

Subgranular zone

Substantia nigra pars compacta

Sub-ventricular zone

Total Ghrelin

Transmembrane domain

Un-acylated Ghrelin

Wild type 


\section{Chapter 1 General Introduction}

\subsection{Ghrelin}

Ghrelin is a 28 amino acid orexigenic hormone that is produced by cells in the lining of the stomach, its name comes from the proto-Indo-European word Ghre, which means "growth" or "to grow" (Inui, 2004). This was first described by Kojima et al. in 1999 as a regulator of growth hormone (GH) release. It had previously been discovered that an unknown endogenous ligand acting through a G-protein coupled receptor (GPCR), GHS-R, stimulated the release of GH from the pituitary (Howard et al., 1996). Kojima then revealed that the post-translational addition of octanoic acid to the ghrelin peptide stimulated GH release, indicating that the n-octanoic acid was essential for ghrelin activity (M Kojima et al., 1999).

\subsubsection{Acylation of Ghrelin.}

Ghrelin is a result of proteolytic cleavage of the precursor peptide preproghrelin to proghrelin, which is the native form of the peptide. It is this pro-ghrelin peptide that undergoes a distinctive post-translational alteration, via the addition of an octanoyl group (the process of acylation) to the highly conserved $3^{\text {rd }}$ serine residue of its structure (Figure 1.1). The enzyme that performs this acylation is ghrelin-O-acyl-transferase (GOAT) (Gutierrez et al., 2008; Yang, Brown, Liang, Grishin, \& Goldstein, 2008). Ghrelin exists in two forms, active or acyl-ghrelin (AG) and the, so-called, inactive unacylated form (UAG). UAG is the most abundant form of this hormone, consisting of approximately $90 \%$ of total circulating ghrelin (Nishi, Yoh, Hiejima, \& Kojima, 2011a).

Obestatin is another product of the ghrelin gene that has an opposing effect to ghrelin on appetite, it is anorectic (J. V. Zhang et al., 2005). It is a 23- 
amino acid peptide which is found in the gastrointestinal tract that reduces food intake, body weight and gastric motility, and mediates it's effects through its own GPCR GPR-39 (Lacquaniti, Donato, Chirico, Buemi, \& Buemi, 2011; J. V. Zhang et al., 2008).

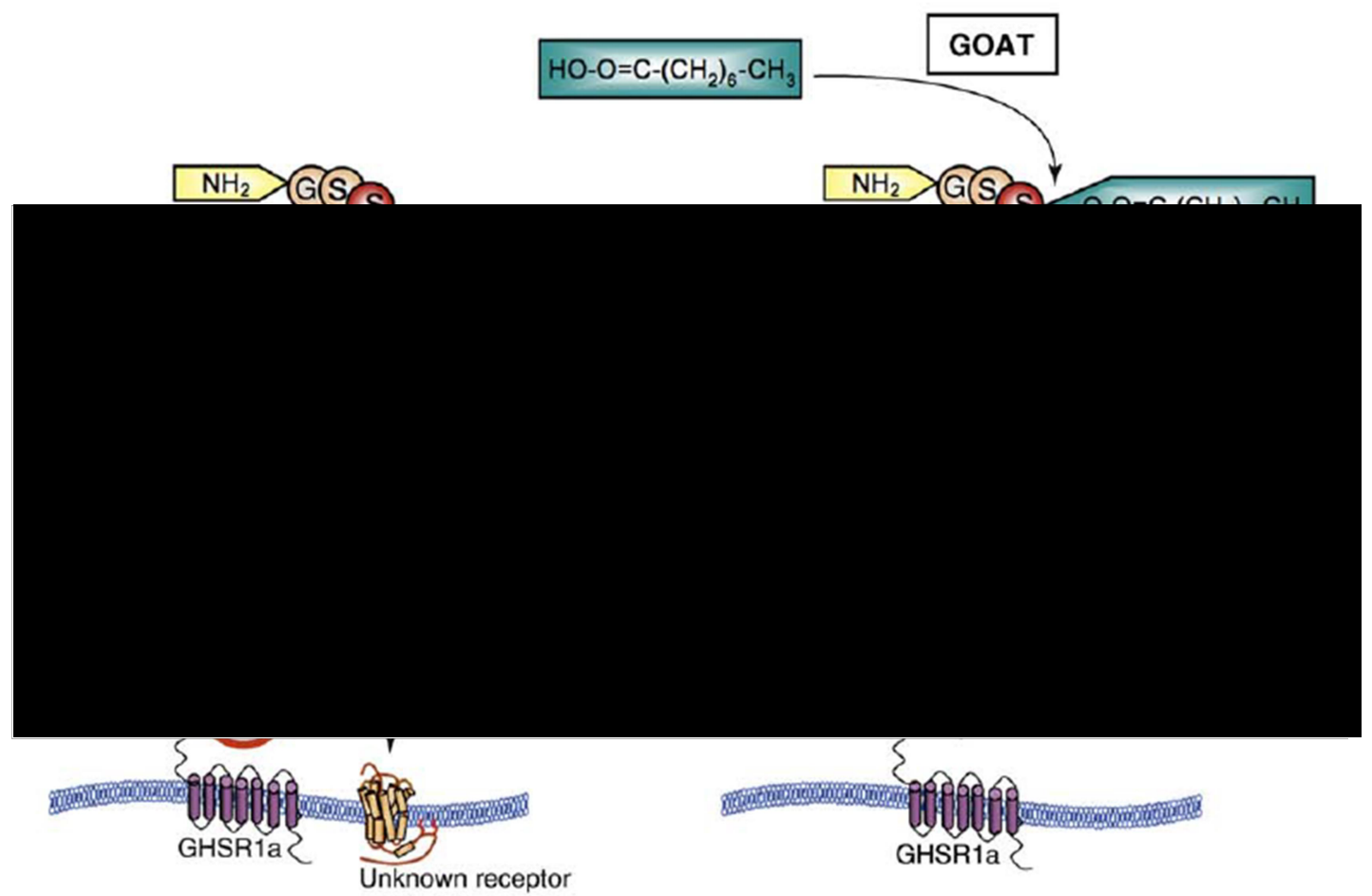

Figure 1.1 The structure of ghrelin and activation by acylation.

Ghrelin comes in 2 forms active (acylated) and inactive (des-acylated). Activation occurs upon the addition of an octanoyl group to the $3^{\text {rd }}$ serine residue, facilitated by the enzyme GOAT. This allows interaction with the ghrelin receptor GHSR1a, the inactive form cannot activate GHSR1a (Taken from Andrews 2011).

GOAT, the enzyme that catalyses the activation of ghrelin was discovered by 2 separate labs in 2008, they both used genome database searches to identify MBOAT4 (membrane-bound o-acyltransferase). The knockdown of MBOAT4 in the medullary carcinoma TT cell line resulted in the prevention of ghrelin acylation, and this was also seen in a knockout (KO) mouse model of MBOAT4, indicating that MBOAT4 was GOAT (Gutierrez et al., 2008; Masayasu Kojima, Hamamoto, \& Sato, 2016; Yang et 
al., 2008). GOAT is a membrane-bound enzyme that has multiple transmembrane domains, and is one of at least 16 acyltransferase enzymes (Hofmann, 2000). Topographically, GOAT has 11 transmembrane domains and a re-entrant loop which are hydrophobic; these are separated by short hydrophilic loops with the N-terminus in the lumen of the endoplasmic reticulum and the C-terminus in the cytosol. It also contains conserved asparagine $\left(\mathrm{Asn}^{307}\right)$ and histidine $\left(\mathrm{His}^{338}\right)$ residues. His ${ }^{338}$ is thought to be involved in the catalytic properties of the active site, whereas $\mathrm{Asn}^{307}$ is thought to be essential for interactions with substrate and transport (Masumoto et al., 2015; Taylor et al., 2013). It is distributed similarly to ghrelin in the gastrointestinal organs, mainly the stomach.

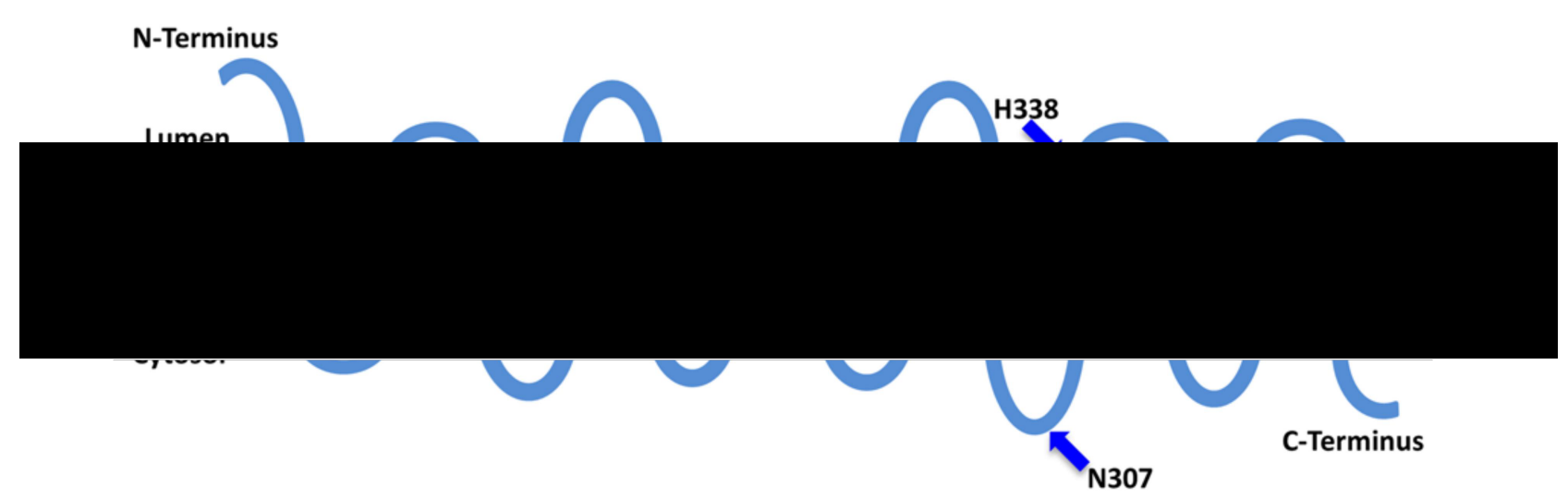

Figure 1.2 Topographic representation of GOAT.

11 transmembrane domains with 1 re-entrant loop, within the membrane of the ER. Blue arrows indicate the conserved $\mathrm{H}^{338}$ in the ER lumen and $\mathrm{N}^{307}$ in the cytosol. Image taken from Masumoto et al. 2015.

Ingested medium chain fatty acids (MCFA) are the predominant source of $n$-octanoic acid (Nishi et al., 2005), suggesting that the acylation of ghrelin may be modulated by dairy products such as milk and oils such as coconut oil and palm kernel oil (Lemarié, Beauchamp, Legrand, \& Rioux, 2016).

\subsubsection{Role in energy homeostasis and orexigenesis}

Ghrelin plays a role in energy homeostasis; it stimulates appetite via orexigenic neurones in the hypothalamus and is expressed in the stomach (M 
Kojima et al., 1999). In a study performed in the laboratory of Tamas Horvath it was shown that immunoreactive ghrelin cell bodies are found in the paraventricular, arcuate, vetro- and dorsomedial hypothalamic nuclei, and in the ependymal layer of the third ventricle, whereas ghrelin knockout animals did not show ghrelin immune-reactive cells in the same areas or the stomach (Cowley et al., 2003). They found that ghrelin was present in axons and that they made synaptic contacts with dendrites from neuropeptide $Y$ (NPY)/ Agouti-related protein (AGRP) and pro-opiomelanocortin (POMC) neurones in the hypothalamus that are part of the appetite regulation pathway. Electrophysiology studies performed on hypothalamic slices treated with 50nM ghrelin, showed an increase in the NPY neuron activity which ceased upon wash-out. This indicated that ghrelin was enhancing the release of NPY and AGRP. Also, ghrelin's effect on POMC neurones was examined using patch-clamp recordings of identified POMC neurones from hypothalamic slices. Ghrelin treatment caused hyperpolarisation, an increase in GABA secretion and a reduction in POMC neurone activation, resulting in a reduction in anorexigenic signalling. Even in the absence of GABA receptor, ghrelin treatment caused the hyperpolarisation of POMC neurones suggesting that this was causing the secretion of another factor, in all probability NPY. This work displayed ghrelin's ability to induce neurotransmitter and orexigenic peptide release to control energy homeostasis (Cowley et al., 2003). The following year Howard Chen also found that NPY and AGRP mediated the orexigenic effects of ghrelin. AGRP/NPY double knockout (KO) mice were used to show that ghrelin treatment did not affect food intake, compared to wild type controls where ghrelin increased food intake by more than $100 \%$. This work supports the knowledge that ghrelin's orexigenic action requires NPY and AGRP $(\mathrm{H}$. Y. Chen et al., 2004).

Ghrelin works in conjunction with another hormone, leptin. Where ghrelin induces orexigenic effects, leptin inhibits appetite by increasing anorexigenic peptide signalling and causing weight loss by decreasing 
appetite and the storage of fats (Farooqi et al., 2001; Licinio et al., 2004). Leptin is produced in adipose tissue, travels through the blood stream and can cross the blood-brain barrier (BBB), where in the hypothalamus, it mediates the effects of anorexigenic peptides such as POMC, brain-derived neurotrophic factor (BDNF), and cocaine-and amphetamine-regulated hormone (CART) and supresses the activity of NPY/AGRP hypothalamic neurones (Klok, Jakobsdottir, \& Drent, 2007).

A recently identified circulating antagonist to ghrelin's action is liverexpressed antimicrobial peptide 2 (LEAP2). This is also expressed in GI organs and the liver, and its release is inhibited by fasting. LEAP2 antagonises ghrelin action by binding directly to the ghrelin receptor and preventing its activation by ghrelin. Therefore, it prevents ghrelin induced food intake, GH release and the maintenance of stable glucose levels during calorie restriction (Ge et al., 2017).

Asprosin is another orexigenic hormone that is expressed in adipose tissue and upon fasting stimulates the release of glucose from the liver (Romere et al., 2016). In a similar way to ghrelin, it can cross the BBB to act on the hypothalamus and elicit a feeding response. It activates AgRP+ neurones to stimulate appetite and inhibit anorexigenic $\mathrm{POMC}^{+}$neurones (Duerrschmid et al., 2017).

The synergy between ghrelin and the recently identified LEAP2 and asprosin, particularly within the CNS, have yet to be elucidated.

\subsubsection{Lessons from Ghrelin KO mice}

The extra-hypothalamic actions of ghrelin can be begun to be understood through the use of ghrelin KO mouse models. Ghrelin KO mice have shown decreases in dendritic spine length and synapse formation. Another finding from the work performed by Sabrina Diano was that ghrelin induced long-term potentiation (LTP) in the hippocampus, this in tandem 
with the increase in synapse formation is important for spatial learning and memory. Following ghrelin replacement in these mice, neurone spine length and density was increased to WT levels and showed a significant improvement in memory based behavioural tasks, including the T-maze foot shock avoidance test and the novel object recognition (NOR) test to measure hippocampal memory. Furthermore, mice that exhibit an Alzheimer's-like disease phenotype (SAMP8 mice) showed improvements in memory retention following peripheral treatment with ghrelin (Diano et al., 2006). In another study, the lab of Seungjoon Park showed with the use of ghrelin KO (GKO) mice that ghrelin was responsible for the stimulation of adult hippocampal neurogenesis (AHN). 8-9 week old C57BL/6 GKO male mice and age-matched wild type controls were treated with saline or ghrelin peptide $(80 \mu \mathrm{g} / \mathrm{kg}$, i.p.) once a day for 8 days. Mice were also administered BrdU $(50 \mathrm{mg} / \mathrm{kg}$ ) twice a day for the final 3 days of ghrelin injections. Immunohistochemical analysis demonstrated that GKO+saline mice had decreases in progenitor cells, proliferating cells, new-born immature and mature neurones, which were all rescued to WT levels with ghrelin treatment. Also, these GKO mice exhibited memory deficits in a Y-maze novel object recognition test, which is hippocampal dependant memory $(\mathrm{Li}$, Kim, Kim, \& Park, 2013).

The KO of ghrelin receptor, GHSR1a, has also demonstrated similar findings with regards to memory function. In a study at the lab of Roy Smith, C57BL/ 6 ghsr KO mice and wild type controls were behaviourally assessed. In rota-rod testing, which measures balance and motor co-ordination by placing mice on a rotating rod, and a hot-plate test, where mice were placed on an hot-plate and their nociceptive response to pain was measured, there was no difference between WT and KO's. Also, KO's showed increased anxiety-like behaviour in the open field test, where locomotor behaviour of the mice was observed by placing them in the centre of an acrylic chamber and measuring their activity in the first 5 minutes, by moving from the centre in significantly less time than WT mice. The KO mice also exhibited 
impairments in contextual fear conditioning (foot shock and freezing time assessed), where $\mathrm{KO}$ mice showed significantly less freezing time compared to WT, 30 days following the initial foot-shock, indicating this memory impairment (Albarran-Zeckler, Brantley, \& Smith, 2012).

These data suggest that ghrelin or synthetic analogues could potentially be used as a therapy in neurodegenerative disorders associated with cognitive decline.

\subsection{The Role of G-Protein Coupled Receptors (GPCR's)}

GPCR's are the most abundant cell surface receptors that convey extracellular signals across cell membranes, which is sub-divided into 4 subfamilies based on their properties; rhodopsin-like A, secretin-like B, frizzled receptors and glutamate/metabopric C receptors (Jastrzebska, 2013). They typically consist of 7 transmembrane (TM) domains connected by varying length intracellular and extracellular loops (ECL). They have conserved elements such as disulphide bonds between ECL2 and TMIII, D[E]RY motif atop of TMIII and the NPxxY motif in TMVII. These highly diverse GPCR's interact with a small quantity of heterotrimeric $G$ proteins. These molecular switches consist of 3 subunits ( $\alpha, \beta$ and $\gamma$ ) with a nucleotide binding pocket within the a-subunit where the conversion of GDP to GTP occurs, causing conformational changes and the G-protein to dissociate from the GPCR, allowing downstream signalling to happen (Jastrzebska, 2013; Katrich, Cherezov, \& Stevens, 2013). GPCR's can function as monomers; they have been shown to homo- and hetero-dimerise/oligomerise. This dimerization allows for alteration and efficiency of signal transduction, and allow for more control of physiological functions (Milligan, 2007). 


\subsubsection{Ghrelin Receptor}

Ghrelin has two GPCR's, the main functioning receptor, growth hormone secretagogue receptor 1a (GHSR1a) and a truncated splice-variant form of the receptor, GHSR1b. GHSR1a was first characterised before the discovery of its endogenous ligand, ghrelin. GHSR was established as a receptor that controlled GH release in the pituitary and hypothalamus (Howard et al., 1996). The functionally active GHSR1a is predominantly expressed in the anterior lobe of the pituitary gland and in the arcuate nucleus of the hypothalamus, an area important for ghrelin's orexigenic activity (Camiña, 2006). It is through GHSR1a that ghrelin mediates its effects on energy homeostasis. GHSR1a is expressed on NPY/AGRP expressing neurones in the arcuate nucleus which promote food intake (Willesen, Kristensen, \& Rømer, 1999).

GHSR1b, the truncated form of the receptor that only has 5TM's, has been shown to hetero-dimerise with GHSR1a, causing conformational changes that prevent GHSR1a activation by ghrelin binding (Leung et al., 2007). In vitro (HEK-293 cells) GHSR1a forms homo-dimers, but when GHSR1b mRNA expression exceeds GHSR1a mRNA expression analysed by quantitative real-time RT-PCR, it reduced the cell surface expression of the functionally active receptor, thus having a dominant-negative effect. The absence of TM6 and TM7 in GHSR1b may affect ghrelin's ability to recognise the receptor due to an altered binding pocket, or are required for direct interaction (Leung et al., 2007; Mary et al., 2013). GHSR1b also has been shown to modulate GHSR1a in striatal and hippocampal neurone cell cultures, and with HEK-293T cells. These were used to show that low levels of GHSR1b expression with GHSR1a promotes GHSR1a trafficking to the plasma membrane, whereas higher GHSR1b expression prevented GHSR1a function. Also, in these striatal and hippocampal neurone cultures GHSR1aGHSR1b heteromers interacted with dopamine-1 receptor (D1R) allowing for 
Gs/olf (G-proteins Gas/Gaolf) protein-dependant ghrelin signalling (Navarro et al., 2016).

\subsection{Calorie Restriction}

The beneficial effects of calorie restriction (CR) are conserved across species. CR has been shown to increase life-span 2- to 3-fold in yeast, worms and flies, and increase life expectancy in mice by $30-50 \%$. A similar trend has been reported in monkeys, but the effect in humans has yet to be determined, however it does aid in the prevention of diabetes, obesity and hypertension and reduces the risk of cancer and heart disease (Fontana, Partridge, \& Longo, 2010). In another study CR has led to the decline in loss of memory and learning function and other age-associated deficiencies in humans (Witte, Fobker, Gellner, Knecht, \& Floel, 2009). Further studies in rhesus monkeys have shown that $\mathrm{CR}$ can reduce the prevalence of age-related deaths when the regime is introduced at early age or in later adult life (Colman et al., 2014; Mattison et al., 2017). Also, in mouse models of ageing and neurodegenerative disease, restricting diet has had beneficial effects on protecting the loss of hippocampal, cortical and striatal neurones by increasing the production of BDNF (W Duan, Lee, Guo, \& Mattson, 2001). A better understanding of $\mathrm{CR}$ physiology could help develop $\mathrm{CR}$ mimetics for the treatment of age-related disorders.

\subsubsection{CR and Ageing}

With an increasingly aged population and an increase in the incidence in dementia and neurodegenerative disorders, CR has been shown to reduce the effects of ageing on the production of new neuronal progenitor cells. A study in Nestin-GFP reporter mice (6 months old) showed that increased exposure to a CR regime; 1 week of $80 \%$ of ad-libitum fed mouse consumption then $60 \%$ of ad-libitum fed for 12 months $(n=12)$, resulted in an increase in 
dividing cells in the DG of the hippocampus. Mice were injected with the Sphase markers, CldU (5-chloro-2'-deoxyuridine, $128 \mathrm{mg} / \mathrm{kg}$ ) 24 hours prior and IdU (5-iodo-2'-deoxyuridine, $173 \mathrm{mg} / \mathrm{kg}$ ), 2 hours prior to terminal anaesthesia. Immunohistochemical analysis of Nestin-GFP ${ }^{+} \mathrm{CldU}^{+}$and Nestin-GFP+IdU+ cells in the SGZ of the DG showed a 2-fold increase, and 5fold increase, respectively, of new-born neural progenitor cells, in aging mice that underwent 12 months CR compared to ad-libitum fed controls. However, analysis of immature neuronal cells $\left(\mathrm{DCX}^{+}\right)$did not show the same response, suggesting that $\mathrm{CR}$ increases the proliferative capacity of progenitor cells (J.H. Park et al., 2013). Prolonged CR has also been shown to illicit improvements in memory function. A study performed by Angela Kuhla showed that C57BL/ 6 mice (4-week old) that underwent $4(\mathrm{n}=10), 20(\mathrm{n}=$ $10)$ and 74 weeks $(n=10)$ of $C R$ feeding $(60 \%$ of food consumed by ad-libitum fed controls) had improvements in working memory, tested using the morris water maze (MWM). MWM specifically tests for spatial learning, which is hippocampal specific, where mice have to locate a submerged platform in opaque water. Ad-libitum fed mice showed an age-related decrease in their performance in the task, whereas those that underwent long-term CR (74 weeks) had improved performance scores (an increase in platform crossings). A separate group of mice that were introduced to a $\mathrm{CR}$ regime later on, at 66 weeks old for 12 weeks $(n=10)$, did not demonstrate the same effect. This indicates that life-long $\mathrm{CR}$ is required for improved memory function with increased age (Kuhla et al., 2013).

\subsubsection{CR and Ghrelin}

$\mathrm{CR}$ is thought to mediate its beneficial effects on the brain via hippocampal plasticity and neurogenesis, and one possible mediator is ghrelin (Zane B Andrews, 2011). Ghrelin regulates aspects of hippocampal neurogenesis; ghrelin $(80 \mu \mathrm{g} / \mathrm{kg}$ for 8 days) administered to male 8-week old adult mice via intra-peritoneal injection, and the effects on neural progenitor cell differentiation and proliferation were quantified within the dentate 
gyrus (DG) of the hippocampus. Ghrelin significantly increased the number of new-born cells and immature neurones in the sub-granular zone (SGZ) of the DG following treatment. This suggests that administration of ghrelin increases cell proliferation, and could potentially be used therapeutically for disorders involving learning and memory (Moon, Kim, Hwang, \& Park, 2009). Much work on ghrelin regulation of adult hippocampal neurogenesis $(\mathrm{AHN})$ has been done in the lab of Seungjoon Park. Using ghrelin KO mice they found that genetic ablation of ghrelin caused a reduction in progenitor cell proliferation in the SGZ which was restored to wild-type levels upon ghrelin treatment. These $\mathrm{KO}$ animals also had deficits in hippocampal dependent memory tasks (Y-maze and NOR test), which were reversed upon ghrelin treatment (50 $\mu \mathrm{g} / \mathrm{kg} /$ day, for 28 days) (Li, Chung, et al., 2013). As restriction of diet has been shown to positively modulate new adult-born cell survival (Jaewon Lee, Seroogy, \& Mattson, 2002; H. R. Park \& Lee, 2011), the S. Park group provided a link between CR, ghrelin and AHN. Again, using ghrelin KO mice and wild type (WT) littermates (male 8-10 week old) that were CR'ed (every other day fed, 24 hour fast followed by 24 hour fed) or ad libitum fed for 3 months. They reported that stomach and hippocampal levels of ghrelin were increased in WT mice that underwent CR, and that survival of new adult-born cells was enhanced in WT CR'ed mice, which was not observed in ghrelin KO's (Kim et al., 2015).

\subsection{Adult Neurogenesis}

Adult neurogenesis is the process by which new neurones are produced in the adult brain and was first recognised in rats by Joseph Altman in 1965. Using autoradiographs and histological analysis of rats that had been administered intraperitoneal (i.p.) injections of thymidine- $\mathrm{H}^{3}$ to label dividing cells, they found labelled cells in the basal granule cell layer (GCL) of the dentate gyrus (DG) as shown in Figure 1.3. In this study they examined multiple brains of male rats ranging from 6 hours to 9 months of 
age, where they discovered a decrease in the number of labelled cells with increasing age of the animals. The higher cell numbers in younger rats was attributed to a higher rate of cell proliferation which declined with age, but did not cease (Altman \& Das, 1965). To examine this further, in 1977 Michael Kaplan and James Hinds performed a study to identify neurogenesis in the DG of the adult rat. They used injections of thymidine- $\mathrm{H}^{3}$ in 3 month old rats that lived for 30 days following injection, and used electron microscopy to identify neurones and labelled granule cells in the DG and olfactory bulb $(\mathrm{OB})$. Contesting the notion that the adult brain remains static in adulthood (Kaplan \& Hinds, 1977). Studies have also used the thymidine analogue 5bromodeoxyuridine (BrdU) that incorporates into the DNA of dividing cells in the S-phase of the cell cycle (del Rio \& Soriano, 1989).

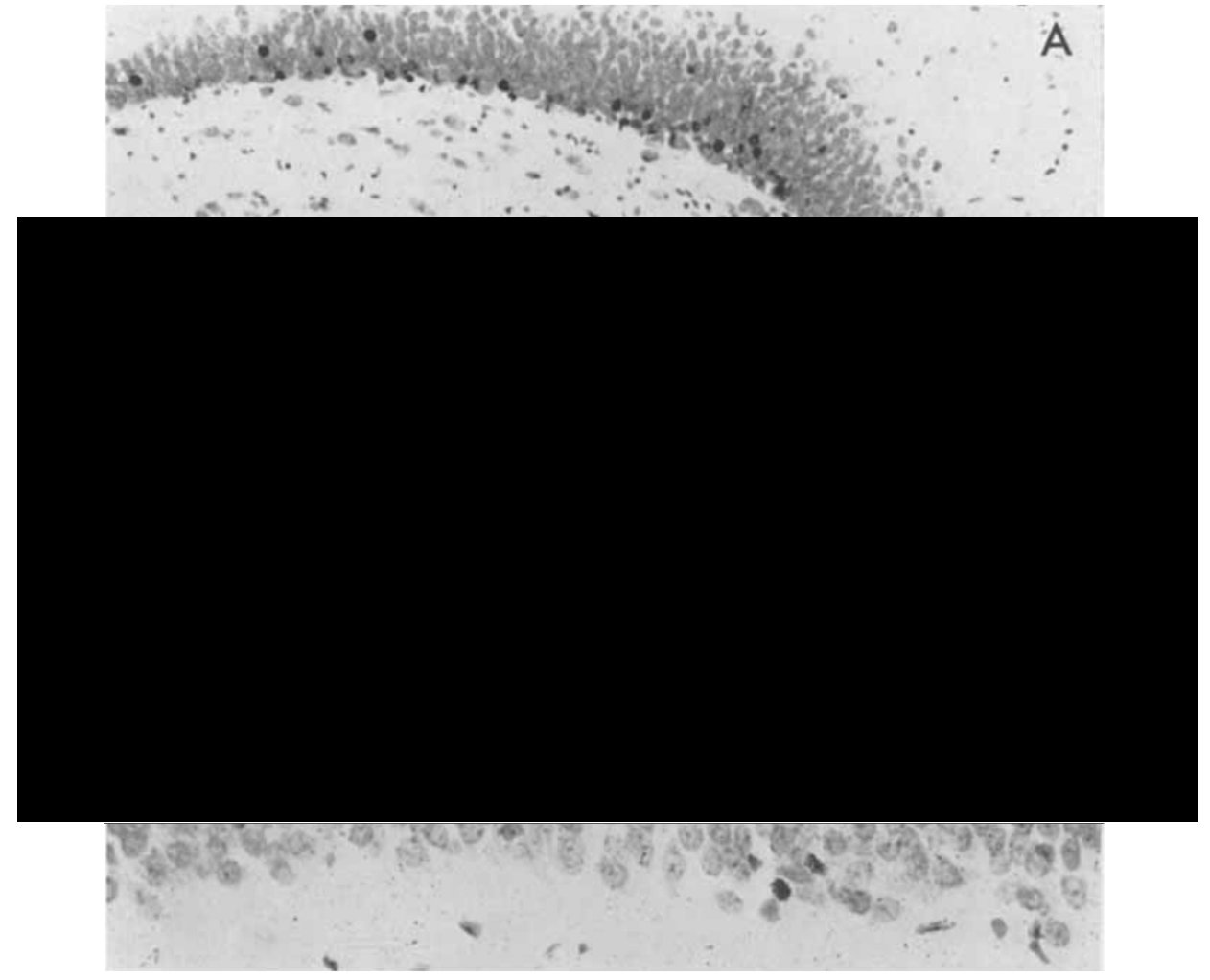

Figure 1.3. Autoradiographs of the dentate gyrus depicting thymidine- $\mathrm{H}^{3}$ cells in rats.

10 day old rat injected with thymidine- $\mathrm{H}^{3}$ and sacrificed 2 months following the last injection. Granule cells are labelled in the DG (A) and most notably in the basal GCL (B). A = 100x; A = 256x. Image taken from (Altman \& Das, 1965). 
This technique has allowed for the advancement of AHN analysis, due to the ability to use confocal microscopy and immunofluorescent detection. A study from the lab of Fred Gage looked at the human brains ability generate new neuronal cells through life, and they achieved this by using the post-mortem hippocampal brain tissue of cancer patients $(n=5$, aged 57 - 72) who had been administered one dose of BrdU (250mg; $2.5 \mathrm{mg} / \mathrm{ml}, 100 \mathrm{ml}$ ) as part of their treatment. Immunohistochemical analysis revealed $\mathrm{BrdU}^{+}$cells in the human DG (compared to a non-BrdU treated cancer control), the same neurogenic area seen in adult mice and rats. Double-fluorescent labelling revealed that these new-born cells also expressed mature neuronal marker NeuN, confirming neurogenesis (Eriksson et al., 1998). More recently, carbon-14 dating has been used to birth-date new neurones in the adult human brain. Due to nuclear weapons testing in the 1950's and 1960's the levels of atmospheric carbon-14 were greatly increased. Following the ban of above-ground nuclear testing in 1963, carbon-14 levels have decreased. In this work from Jonas Frisen's lab, tissue samples from individuals born before and after the nuclear bomb tests showed that new neurones are produced in the adult human striatum and hippocampus, at the same rates seen in rodents (Ernst et al., 2014; Spalding et al., 2013; Spalding, Bhardwaj, Buchholz, Druid, \& Frisén, 2005).

\subsubsection{Adult Hippocampal Neurogenesis}

AHN occurs in 2 distinct regions in the adult mammalian brain, the sub-ventricular zone (SVZ) of the lateral ventricles where new-born neurones migrate along the rostral migratory stream and integrate into the $\mathrm{OB}$, and the SGZ of the DG where new-born neurones migrate across the GCL and integrate into existing hippocampal circuitry (Figure 1.4). The hippocampus is important for episodic memory, which involves the capacity to learn, store and recollect information (Dickerson \& Eichenbaum, 2010). The SGZ of the DG is highly neurogenic mainly due to the two types of neural progenitor cells being in such close proximity to the granule cells (GCs) in the DG, 
which consists of both immature and mature neurones. There are many other cell types in this unique microenvironment, such as astrocytes, that may help promote the differentiation and integration of new-born cells into the neuronal circuitry (Zhao, Deng, \& Gage, 2008a). There are 4 phases of AHN; precursor cell phase, early survival, post-mitotic maturation and late survival (Kempermann, Jessberger, Steiner, \& Kronenberg, 2004; Steiner et al., 2006). During these phases the developing cells express different protein markers and have different morphologies. In the precursor cell stage the progenitor cells have a radial glial-like morphology and express markers such as Nestin and GFAP, and are classified as type 1 neural stem/progenitor cells (NSPCs). Through expansion and survival these cells then become less glial-like and express markers such as Sox2, type 2 NSPCs. Following post-mitotic maturation these early mature cells begin to extend axons and dendrites, and express the marker for immature neurones, doublecortin (DCX). After the late survival and maturation stages, these cells have become mature dentate granule neurones whose axons extend out to the CA3 and dendrites into the molecular layer, and express the protein marker NeuN (Hanson, Owens, \& Nemeroff, 2011; Kempermann, Song, \& Gage, 2015).

The mechanisms in which new adult born mature neurones are incorporated into the existing neuronal network are recently better understood. Tassilo Jungenitz found that at the mature stage of new adultborn granule cell development (21 days onwards), these cells became synaptically integrated, and used markers of synaptic activity and plasticity, immediate-early genes (IEGs) that code for transcription factors e.g. c-Fos and Arc, and DCX expression to decipher this. 8-13 week old Sprague Dawley rats were administered a 2 hour high frequency stimulation (HFS) to induce LTP, which resulted in an increase of both c-Fos ${ }^{+}$and $\mathrm{Arc}^{+}$expression in calbindin ${ }^{+}$mature granule cells in the DG. They also showed that immature $\mathrm{DCX}^{+}$cells do not express c-Fos or Arc, but mature DCX-cells are c-Fos ${ }^{+}$. Following injection of BrdU $(200 \mathrm{mg} / \mathrm{kg})$ and 2-hour HFS, BrdU+ cells that also expressed c-Fos and Arc were observed initially at 21 days post- 
injection, and increased with advancing cell age, reaching $75 \%$ at 35 days post-injection. The same pattern was observed when cells were analysed for Egr-1 expression. These data indicate that as new adult-born dentate granule cells mature they become more synaptically active (Jungenitz, Radic, Jedlicka, \& Schwarzacher, 2013). The IEG Egr-1, also known as zif268, is also involved in the integration of new-born neurones. In a study by Alexandra Veyrac, zif268-KO mice exhibited increased cell death of new adult-born neurones 2-3 weeks after their birth, defects in dendrite and dendritic spine development, and disruption in the recruitment of new adult-born neurones following spatial learning and memory tasks, resulting in impaired longterm memory (Veyrac et al., 2013).

Interestingly, it has been shown that ghrelin treatment induces c-Fos and Egr-1 expression in the arcuate nucleus of fasted and normally fed rats. Male Wistar rats were administered saline $(0.2 \mathrm{ml}, \mathrm{n}=12)$, ghrelin $(10 \mu \mathrm{g}, \mathrm{n}$ $=14)$ or unacylated ghrelin $(10 \mu \mathrm{g}, \mathrm{n}=12)$ via jugular vein catheter injection, where half of each group were also fasted for 48 hours prior to treatment, and killed 90 minutes later. Immunohistochemical analysis revealed that ghrelin treatment in both fasting and normally fed groups resulted in an increase the number of c-Fos ${ }^{+}$and Egr- $1^{+}$cells in the arcuate nucleus (Hewson \& Dickson, 2001).

Recently it has been shown that there are similarities between embryonic and post-natal DG neurogenesis. Single-cell RNA sequencing was performed in micro-dissected DG's from mice ranging from embryonic day (E) 16.5 to post-natal day (P) 132. Data analysis unearthed 4 distinct groups of cells; immune cells such as microglia and macrophage, vascular cells such as endothelial cells, glial cells, and neuronal cells, with dentate granule cells being the predominant cell type (immature granule cells, radial glial-like cells (RGL) and mature granule cells). The types of cells did not change over time, but the quantity of each cell type differed and their position in the DG. RGL's of the DG seemed to be mostly quiescent during development, but 
after development they entered the cell-cycle and become neuronal intermediate progenitor cells (nIPC's) and commit to a neuronal lineage. This process was conserved from E16.5 to P132 (Hochgerner, Zeisel, Lönnerberg, \& Linnarsson, 2018).

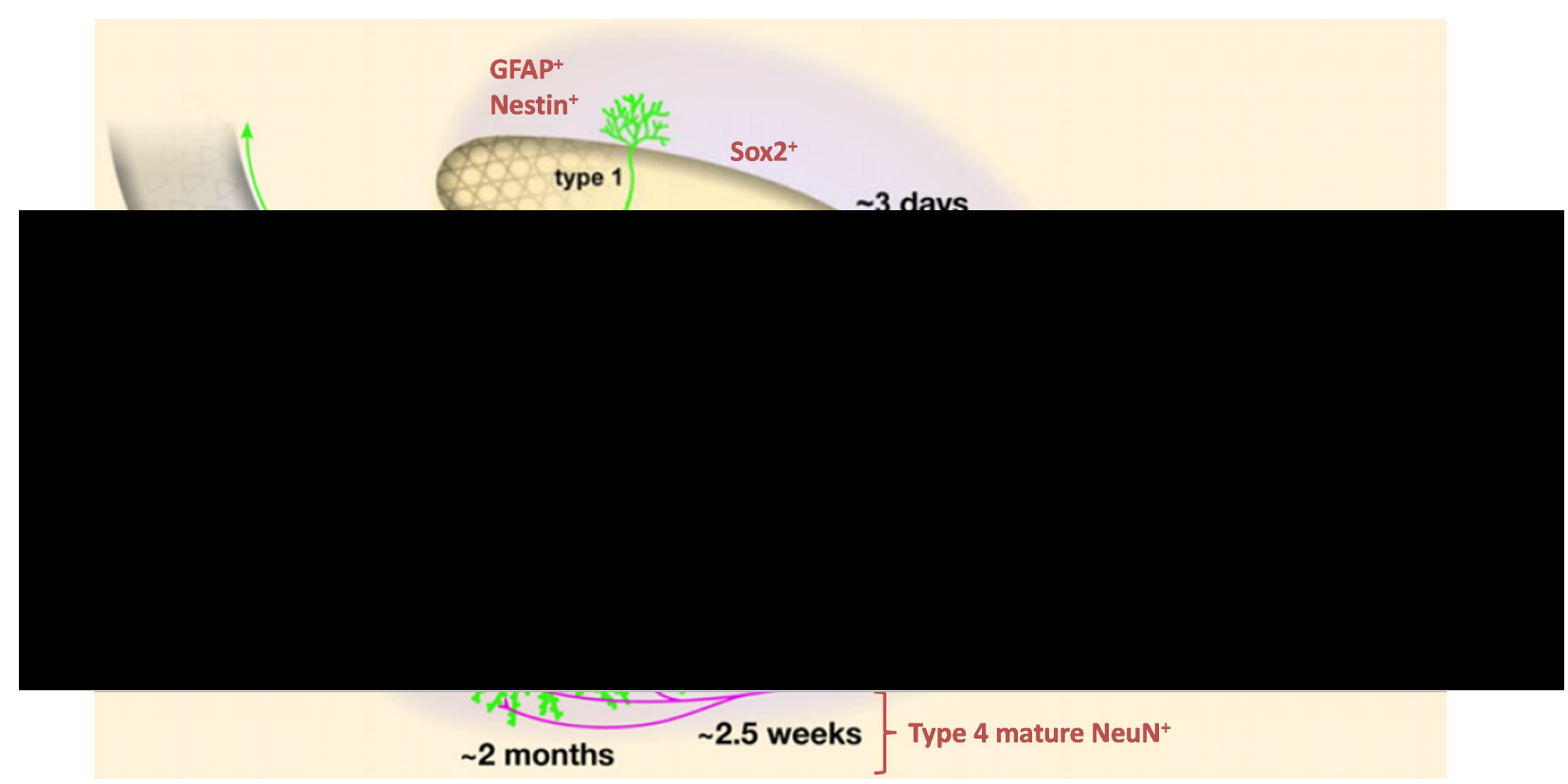

Figure 1.4 Process of Neurogenesis in the Dentate Gyrus.

Type 1 (radial glial-like) and type 2 (non-radial) progenitor cells, progress through the process of neuronal development which takes approximately 2 months, from the SGZ and up through the GCL before integrating into existing circuits. Image taken from Zhao et al. 2008 with annotation.

\subsubsection{Regulation of AHN}

AHN is enhanced by exercise and enriched-environments. Exercise increases the rate of proliferation of precursor cells in the SGZ of the DG and enriched-environments promote their survival (Fabel et al., 2009). Enriched environments involved stimulation from innate objects in combination with social stimulation (Kempermann, Kuhn, \& Gage, 1997). In a study performed in the laboratory of Gerd Kempermann, it was shown by co-labelling new cells with the thymidine analogue BrdU and the mature neurone marker NeuN, that voluntary wheel running alone increased the number of new neurones in murine hippocampi. Moreover, exposure to an enriched 
environment increased their survival. With the combination of both exercise and environmental enrichment, there was a significant increase in the amount BrdU/NeuN expressing cells in the DG, therefore an increase in neurogenesis. It was concluded that physical activity elicits a greater neurogenic response only in conjunction with cognitive stimuli such as an enriched environment (Fabel et al., 2009). Notably, exercise and enriched environment also reduced the formation of amyloid- $\beta$ plaques, reinstated neurogenesis and improved cognition in transgenic mouse models of Alzheimer's Disease (Mu \& Gage, 2011).

Neurogenesis is also affected by anti-depressants. Anti-depressants have been shown to increase hippocampal neurogenesis in human cells and mammals (Taupin, 2006). The anti-depressant Sertraline was incubated with human hippocampal progenitor cells in-vitro for 72 hours during their proliferation phase and for the following 7 days of differentiation. Sertraline increased the number of MAP-2 positive neurones by $28 \%$, but had no effect on immature DCX positive neuroblasts. When these cells were only treated in the proliferation phase to see if treatment induced differentiation, it was shown to have no effect on MAP-2 positive neurones but increased the number the DCX positive immature neuronal cells. When cells were only treated in the differentiation phase, no effect was seen on either cell type. These findings indicated that tricyclic and selective serotonin reuptake inhibitor anti-depressants induced differentiation in proliferating cells. In addition, BrdU labelled cells, following 72 hours of treatment with Sertraline in the progenitor cell phase, revealed a reduction in the number of BrdUpositive cells, supporting the idea that progenitor cells cease proliferation and begin developing into neurones (Anacker et al., 2011). Another study performed by Luca Santarelli also demonstrated the positive effect of antidepressants, in this case Fluoxetine, on AHN. Adult mice $(\mathrm{n}=7-10)$ were treated with fluoxetine or vehicle for 5, 11 or 28 days and administered BrdU $(75 \mathrm{mg} / \mathrm{kg} \times 4)$ on the last day of treatment, before being killed the following day. This resulted in a $60 \%$ increase in BrdU' cells in the DG after both 11 
and 28 days of anti-depressant treatment and a decreased latency to feeding in the novelty-supressed feeding test, compared to vehicle control. This effect was lost following low dose X-ray treatment (5 Gy on days 1, 4 and 8) to the SGZ of the DG, resulting in an $85 \%$ decrease in the BrdU+ cells specific to the SGZ, which were not protected by chronic fluoxetine treatment (28 days, BrdU administered on day 27). The irradiated mice also showed no change in latency to feeding following fluoxetine treatment, compared to nonirradiated controls. These data suggest that fluoxetine action requires AHN to elicit its behavioural effects (Santarelli et al., 2003).

\subsubsection{Role of AHN in Hippocampal-dependent Learning and Memory}

A relationship between neurogenesis and hippocampal-dependent learning and memory has been shown in a study by Sandrine Thuret, using MRL/MpJ mice that have reduced levels of adult neurogenesis and agematched C57BL/ 6 control mice (10 week old, $\mathrm{n}=10$ per group). In order to examine neurogenesis in these mice, BrdU was administered $(50 \mu \mathrm{g} / \mathrm{g})$ daily for 6 days, with analysis taking place 24 hours and 4 weeks after the last injection. Immunohistochemical analysis in the DG showed that MRL/MpJ mice had $60 \%$ fewer BrdU ${ }^{+}$cells than C57BL/ 6 controls 24 hours after the last BrdU injection; interestingly this difference was not seen in the SVZ. At 4 weeks post final BrdU injection, MRL/MpJ mice had 75\% fewer BrdU' cells compared to controls and that $80 \%$ of those surviving cells in the DG also expressed the mature neuron marker, NeuN. Therefore, MRL/MpJ mice produce $75 \%$ fewer neurones in the DG compared to C57BL/6 mice. Spatial memory was tested via the MWM. Testing was divided into blocks consisting of 4 separate trials; the first block of 4 trials was performed with a visible stage. The following 12 blocks were performed with the platform hidden. The platform was then removed 24 hours after the last block of trials and in the actual test period that followed, the time spent in the platform area was measured. The MRL/MpJ mice spent less time in the platform area compared to C57BL/6 controls, indicating that spatial memory was 
impaired. Recognition memory was also tested with the visual-paired comparison task, where exploration time of a novel object was monitored. MRL/MpJ mice spent 50\% less time exploring a novel objection, 24 hours after exploring 2 similar objects, whereas C57BL/6 controls spent most of their time exploring the novel object. These results indicating that hippocampal neurogenesis is associated with learning and memory function (Thuret, Toni, Aigner, Yeo, \& Gage, 2009). AHN has also been shown to conserve hippocampal memory function by the modulation of LTP. Analysis in Wistar ST rats showed that when the hippocampal LTP network was artificially saturated, rats demonstrated impaired memory function in response to contextual fear conditioning (CFC, using foot-shock to induce hippocampal-dependent fear memory). With the decay of LTP, improvements in the capacity of memory function were observed. Also, neurogenesis was ablated by x-ray irradiation and resulted in a delay in memory capacity recovery, as these rats displayed lower freezing times in the CFC task and lower rate of neurogenesis (fewer BrdU ${ }^{+} \mathrm{NeuN}^{+}$cells in the DG). In contrast, irradiated rats analysed in a voluntary wheel exercise, which was used to boost neurogenesis, showed an increased rate of LTP saturation decay and recovery in memory function when subjected to CFC. These results show a maintenance function of AHN on hippocampaldependent memory (Alam et al., 2018). In humans, AHN has been shown to reduce the interference in memories when taking part in a pattern separation task following aerobic exercise. Healthy young adults $(n=13, B M I \leq 25)$ that led sedentary lifestyles participated in a 6-week course of high intensity interval training to improve aerobic fitness. Following this 6-week period, participants underwent cognitive testing. Those who responded to the exercise regime ( $8 \%$ increase in peak oxygen uptake) underwent visual neurogenesis-dependent pattern separation testing, where participants were shown 88 images of everyday objects, of which 16 were repeated images, 16 were similar (lures) and 24 were not related (foils). Participants were shown these images at random (2500ms each). After a delay a second set of 112 
images was presented (48 repeated, 48 lures and 16 foils). The ability to distinguish between similar lures was increased in these responders by $12 \%$, compared to testing before the exercise regime. Participants that did not respond to exercise did not show any increase in performance (Déry et al., 2013).

In terms of the causative effects of $\mathrm{AHN}$ on cognitive function, this has been demonstrated by Amar Sahay. By increasing AHN, they showed a significant improvement in pattern separation memory. The increase in newborn neurones was achieved by the genetic knockdown of the pro-apoptotic gene Bax in mice, which resulted in increased new-born neurone survival. Mice with this augmented AHN demonstrated enhanced performance in pattern separation tasks (Sahay et al., 2011). Conversely, AHN ablation in mice by focal $x$-irradiation, which resulted in a significant decrease in the immature neuronal cell pool, showed a pronounced decrease in the ability of mice to discern between stimuli with low spatial separation in a pattern separation task (Clelland et al., 2009).

\subsection{Cognitive Decline and Ageing}

Cognitive decline is part of the normal ageing process, and comes with increased societal, monetary and personal burdens. It has been shown across species that ageing elicits changes in memory processes. Episodic and spatial memory declines with age, making it more difficult to navigate environments (Leal \& Yassa, 2015). The ability to distinguish similar contexts is also reduced, with older adults showing reduced efficiency in pattern separation tasks (Holden, Hoebel, Loftis, \& Gilbert, 2012). Synaptic inputs into the DG and CA3 decline, indicating lesser synaptic activity (Leal \& Yassa, 2015). Structural changes also occur in the brain due to ageing, including a reduction in grey matter and white matter volume caused by neuronal death and diminishing the communication between hippocampal structures. The accumulation of protein aggregates such as amyloid- $\beta$, 
associated with Alzheimer's disease (AD), exacerbates neuronal death in the brains of non-AD aged individuals (Deary et al., 2009; Harada, Love, \& Triebel, 2013) and is thought to contribute to cognitive decline (Miller et al., 2017).

As we have an ever-increasing aged population, the prevalence of cognitive decline and neurodegenerative disorders increases. Parkinson's disease (PD) is the second most prevalent neurodegenerative disorder that currently affects approximately 145,000 people in the UK, that's 1 person diagnosed in every 350 adults (Parkinson's UK (www.parkinsons.org.uk), 2018). PD is characterised by the progressive loss of dopaminergic (DA) neurones from the substantia nigra pars compacta $(\mathrm{SNpc})$ that project to the striatum, which are located in the mid-brain, and leads to a deterioration of motor function through mechanisms that are still unknown. The most recognisable symptoms of PD are resting tremor, slowness in movement and stiffness. There are pre-motor dysfunction characteristics such as weight loss, reduction in appetite and disruption of sleeping patterns. Due to these symptoms arising prior to those that involve motor function, the suggestion is that the initial pathogenic action may transpire outside of the nigrostriatal DA pathway or the pathway controls other aspects of physiology (Bayliss \& Andrews, 2013).

\subsubsection{Parkinson's disease, AHN and Cognitive Decline}

In a 20 year longitudinal study, one of the largest studies performed on PD patients, $83 \%$ of patients that survived the study developed dementia (Hely, Reid, Adena, Halliday, \& Morris, 2008) and most recently 25-30\% of PD patients have dementia associated to PD (Davis \& Racette, 2016; Willis et al., 2012). This indicates that cognitive decline and dementia in PD is an issue that requires more research.

The loss of dopaminergic neurones, which is characteristic of PD, impairs proliferation of precursor cells in the SGZ of the DG in human PD post- 
mortem brains. Dopamine innervation and denervation was showed to affect cell proliferation in the DG. MPTP-treated C57BL/6 mice, which induces SGZ dopaminergic fiber (DAT) loss, were also administered BrdU (8x 50 $\mathrm{mg} / \mathrm{kg}$ every 6 hours). Immunofluorescent staining revealed that new-born cells express DAT, and that the number of $\mathrm{PCNA}^{+}$precursor cells in the DG were significantly reduced in MPTP-treated mice compared to controls, which was reversed 70 days following MPTP administration. Immunohistochemical analysis of adult human DGs revealed that compared to controls $(\mathrm{n}=3), \operatorname{PD}(\mathrm{n}=3)$ and PD with cognitive impairment $(\mathrm{n}=5)$ brains showed significantly reduced numbers of Nestin ${ }^{+}$and $\beta$-III-tubulin ${ }^{+}$ cells, indicating reduced levels of precursor cell proliferation in the DG. These data indicate that reduced AHN in the PD brain may lead to cognitive decline (Höglinger et al., 2004).

Cognitive deficits in PD have been associated with impairments in AHN. A study performed by Keith Wesnes and David Burn showed that PD patients had a significant decrease in object pattern separation performance, a task specific for the hippocampus and DG neurogenesis. A cohort of 72 PD patients (27 female and 45 male, mean age of 70.1) took part in the study as well as 62 age and gender matched controls. The object pattern separation task involved the presentation of 20 images of everyday objects ( 1 shown every 3 seconds) with a 12 minute interval followed by presentation of 20 novel images mixed with the original set that were very similar. Participants were required to press either a YES or NO button with regards to whether the image was a novel or an original image. PD patients showed no difference to controls in recognising the original images, but showed a significant decrease $(p=0.0001)$ in the ability to discern between the highly similar images, which is indicative of impaired DG function and potentially neurogenesis (Wesnes \& Burn, 2014). 


\subsubsection{Parkinson's disease and Ghrelin}

Ghrelin has been shown to have a neuroprotective effect in mouse models of PD (Jiang, Li, Wang, \& Xie, 2008). Andrews et al. 2009 showed that GHS-R was present in the SNpc and that administration of ghrelin increased both dopaminergic neurone firing in this region and the number of mitochondria in these cells. PD-like symptoms were induced in mice with 1-methly-4phenyl-1,2,5,6 tetrahydropyridine (MPTP) leading to dopaminergic neurone loss. However, ghrelin pre-treatment resulted in a marked decrease in dopaminergic neurone loss in the SNpc of these mice. As ghrelin has shown to exhibit a neuroprotective effect in rodent models of PD, serum total ghrelin levels were measured in human PD patients after fasting and postprandially. PD patients $(n=39$, aged 62-73) had a slower recovery in ghrelin levels compared to a control group ( $n=20$, aged 55-63). As PD patients often have impaired gastric motility, there may also be a disruption in the secretion of ghrelin that may increase the incidence of dopaminergic neurone damage. These pre-clinical and clinical data suggest that ghrelin may represent a possible biomarker for PD (M. M. Unger et al., 2011). Further work is needed to validate these findings in humans.

\subsection{Aims and Hypothesis}

As discussed, several published studies have demonstrated the beneficial effects of CR on hippocampal function. Work from the lab of Mark Mattson showed that dietary restriction, in the form of alternate day feeding, promoted survival of new adult born cells in the mouse DG and increases the level of the neurotrophic factor, BDNF (W Duan et al., 2001; Jaewon Lee et al., 2002). Also, CR has been shown to elicit improvements in age-related cognitive decline in a mouse model of Alzheimer's disease, with longer-term $\mathrm{CR}$ inducing positive effects on behaviour impairments in aged 3xTgAD mice. $\mathrm{CR}$ was shown to improve exploratory behaviour in these mice by 
showing greater distance travelled in an open field test compared to controls, as well as having reduced levels of amyloid-beta and phosphorylated-tau proteins (Halagappa et al., 2007).

Ghrelin has been shown to enhance cognitive performance by crossing the blood-brain-barrier and improving the density of synapses formed in the hippocampus. This in turn enhanced spatial learning and memory in mice administered ghrelin or ghrelin mimetic, and rescued memory impairments observed in ghrelin knocked-out mice (Diano et al., 2006). Similarly, ghrelin improved long-term memory function in rats when administered directly to the hippocampus prior to behavioural testing (Carlini, Ghersi, Schiöth, \& de Barioglio, 2010). Ghrelin agonists have also been shown to illicit positive effects on cognitive function in models of Alzheimer's disease. A study examining the effect of the ghrelin agonist, LY444711, on the TgAPPSwDI mouse model showed that it induced spatial memory improvements in a water maze task and also resulted in a reduction in amyloid-beta and inflammation markers (Dhurandhar, Allison, van Groen, \& Kadish, 2013). Similar findings were also demonstrated in a further study using the LY44471 compound and TgAPPSwDI mouse model (Kunath et al., 2015).

Little is known about the function of un-acylated ghrelin, even though it makes up approximately $90 \%$ of circulating ghrelin (Nishi, Yoh, Hiejima, \& Kojima, 2011b). However, UAG has an antagonistic effect compared to acylated ghrelin in downregulating gluconeogenesis in hepatocytes (Gauna et al., 2005) and reducing the insulin secretion response in humans (Broglio et al., 2004). Whilst it appears to have no effect on LTP and synaptic plasticity compared to acylated ghrelin (L. Chen et al., 2011), it is not known whether UAG regulates AHN.

The non-motor symptoms of PD could be attributed to a dysfunction in the ghrelin system and evidence has shown that PD patients display several symptoms that would make the investigation of blood plasma, for a potential biomarker, worthy. Along with motor dysfunction in PD, patients experience 
gastrointestinal dysfunction and impaired gastric motility (Pfeiffer, 2003; M. M. Unger et al., 2010). They also experience weight loss (Fiszer et al., 2010; Lorefält, Toss, \& Granérus, 2009) and loss in appetite (Aiello, Eleopra, \& Rumiati, 2015). A recent study has also shown that ghrelin's response to feeding is attenuated in PD patients (N. Song et al., 2017), although those that exhibited cognitive impairments were not included. It would be useful for diagnostic purposes to determine whether PD patients that exhibit cognitive impairments also have an attenuated ghrelin response.

Whilst these studies clearly demonstrate the beneficial effects of $\mathrm{CR}$ and ghrelin on hippocampal function, it is not known whether these paradigms modulate AHN. Indeed, little is known about the expression of important ghrelin-axis proteins in the neurogenic niche of the hippocampus. Similarly, little is known about the role of unacylated-ghrelin - the so-called inactive form of the peptide - in regulating hippocampal learning and AHN.

This thesis will test the hypothesis that ghrelin increases AHN and mediates a pro-neurogenic effect of $\mathrm{CR}$ on $\mathrm{AHN}$ and cognitive function. It will also examine whether ghrelin may be used as a potential biomarker of cognitive decline or dementia in humans.

The aims of this thesis are to:

1. Elucidate the expression pattern of key ghrelin-axis proteins in extrahypothalamic brain regions associated with learning and memory, including the neurogenic niche of the hippocampus.

2. Determine whether $\mathrm{CR}$ enhances AHN and memory in a GHSRdependent manner.

3. Determine whether UAG and GOAT modulate AHN.

4. Determine whether circulating levels of key ghrelin-axis proteins represent biomarkers of cognitive decline and dementia in humans diagnosed with PD. 


\section{Chapter 2 General Methods}

\subsection{Tissue preparation}

Mouse brain tissue was collected and underwent intra-cardial fixation with 4\% paraformaldehyde (PFA) (Sigma Aldrich, St Louis, USA), sunk in $30 \%$ sucrose prior to being stored at $4^{\circ} \mathrm{C}$ in the cold room and submerged in PBS (Sigma Aldrich, St Louis, USA) with 0.1\% Sodium Azide (Sigma Aldrich, St Louis, USA), until required for analysis.

\subsection{Tissue Sectioning}

Mouse brain tissue were taken from cold storage and kept from thawing with the use of dry ice. The dry ice was crushed using a pestle and mortar, and the tissue placed in this. The tissue was mounted using Jungs Tissue Freezing Medium (Leica Biosystems) and sectioned using a Freezingstage Microtome (Zeiss, Microm HM 450). Coronal sections at a thickness of $30 \mu \mathrm{m}$ were collected sequentially into 12, 24 or 96-well Nunclon plates. The tissue was collected using a 1:6 or 1:12 ratio, so that each well gives a representation of the whole rostro-caudal extent of the brain (with each section being $180 \mu \mathrm{m}$ or $360 \mu \mathrm{m}$ apart). The tissue sections were maintained in PBS $+0.1 \%$ sodium azide (Sigma Aldrich, St Louis, USA) at $4^{\circ} \mathrm{C}$ for long-term storage and prevention of microbial growth. 


\subsection{Immunohistochemical staining protocols}

All immunohistochemical (IHC) protocols used within this thesis are published and generally used protocols of the free-floating method of IHC staining (Hornsby et al., 2016, 2018; Kent et al., 2015).

\subsubsection{Standard protocol of immunofluorescent staining for multiple targets.}

Mouse brain tissue sections were removed from storage plates and placed into 12-well Nunclon plates containing 1x PBS. Sections were washed 3 times for 5 minutes on a rocker, at room temperature (RT). Sections were permeabilised with the application of methanol (Fisher Scientific, Loughborough, UK) to each well, and the plate placed at $-20^{\circ} \mathrm{C}$ for 2 minutes. The methanol was removed and sections were washed in 1x PBS as above. Following this, tissue was blocked with either $5 \%$ normal goat serum (NGS) in $0.1 \%$ PBS-T (1x PBS + 0.1\% Triton-X) or 5\% normal donkey serum (NDS) in $0.1 \%$ PBS-T, to prevent any non-specific binding of antibodies, for 60 minutes at RT. The block was subsequently removed and the primary $\left(1^{\circ}\right)$ antibodies diluted in $0.1 \%$ PBS-T were applied to the sections and incubated either for 1 hour or overnight, at RT or at $4^{\circ} \mathrm{C}$ in the cold room, on a rocker. Following the antibody incubation, sections were washed as before, and fluorescent secondary $\left(2^{\circ}\right)$ antibody diluted in $0.1 \%$ PBS-T was applied for 30 minutes at RT; the plate protected from light from this point to prevent photo-bleaching. Following this, sections were washed as before in 1x PBS. The next $1^{\circ}$ antibody diluted in $0.1 \%$ PBS-T was applied for 1 hour at RT or overnight at $4^{\circ} \mathrm{C}$. After this incubation, the sections were washed as before, and the appropriate $2^{\circ}$ antibody was applied for 30 minutes at RT.

Finally, the sections were washed as before with 1X PBS, and the sections were mounted on to Superfrost+ slides (VWR International) using 
$1 x$ TBS in a glass petri dish, and cover-slipped with $50 \mu 1$ of Prolong Gold Hardset mountant (P36930, Life Technologies). Slides were left to set overnight at RT in a slide wallet and subsequently stored at $4^{\circ} \mathrm{C}$ until analysed.

\subsubsection{Standard protocol of immunofluorescent staining for multiple targets requiring antigen retrieval (AR), with citrate buffer.}

As stated in the above protocol (2.3.1), brain tissue sections were washed with 1x PBS, permeabilised with methanol and washed a further time with PBS. Due to the nature of some targets they required antigen unmasking with the following antigen retrieval (AR) step. Sodium citrate buffer was applied to the sections in each well and the plate placed in an incubator for 60 minutes at $70^{\circ} \mathrm{C}$. The protocol continues as 2.3.1.

\subsubsection{Standard protocol of immunofluorescent staining for the target Bromo-deoxyuridine (BrdU).}

To allow for the successful staining of the target BrdU, which is located within the nucleus, an alternative antigen retrieval method is required. The protocol starts as stated above (2.3.1) with the following amendments. Following the methanol step, a DNA denaturation step was required to allow the staining of incorporated BrdU; tissues incubated in $2 \mathrm{M}$ $\mathrm{HCl}$ for 30 minutes at $37^{\circ} \mathrm{C}$. The $\mathrm{HCl}$ was removed and Sodium Borate buffer $(\mathrm{pH} 8.4)$ was applied for 10 minutes, on a rocker at RT. The sections were then washed with 1X PBS as described above. 


\subsubsection{Standard method of immunohistochemical staining using Nickel- enhanced Di-amino benezidine (Ni-DAB).}

Mouse brain sections were washed twice in 1X PBS for 10 minutes each, followed by a 10 -minute wash in $0.03 \%$ PBS-T, they were then incubated in 1.5\% Hydrogen Peroxide $\left(\mathrm{H}_{2} \mathrm{O}_{2}\right)$ in 1X PBS for 20 minutes to quench any endogenous peroxidases. Sections were again washed in 1X PBS and 0.03\% PBS-T as described above, after which 5\% NGS/NDS block in $0.03 \%$ PBS-T was applied to the sections and incubated for 60 minutes at RT. Sections were incubated in $1^{\circ}$ primary antibody diluted in $0.03 \%$ PBS-T and 2\% NGS/NDS, this was left to incubate overnight at $4^{\circ} \mathrm{C}$ in the cold room, on a rocker. Sections were washed as before and incubated in $2^{\circ}$ secondary biotinylated antibody diluted in $0.03 \%$ PBS-T and $2 \%$ NGS/NDS, was applied to the sections and incubated for 70 minutes at RT and also covered from light. Following another wash series, a premixed ABC (Avidin-biotin complex) solution was applied $(0.4 \% \mathrm{~A}+0.4 \% \mathrm{~B}$ in $0.03 \%$ PBS-T, premixed for 30 minutes before application); this was incubated for 90 minutes at RT. Subsequently, sections were washed 2 times in 1X PBS for 10 minutes each, and washed in Sodium Acetate (pH6) for 10 minutes. Tissues were reacted in $\mathrm{Ni}-\mathrm{DAB}$ and glucose oxidase solution for between 5-10 minutes. Following this reaction the sections were washed 2 times in 1X PBS.

The sections were mounted onto glass slides as described previously, and allowed to dry overnight. The slides were subjected to a dehydration and delipifying series of 70\% EtOH, 95\% EtOH, 2 times 100\% EtOH and 2 times Histoclear. Sections were placed into these solutions for 3 minutes each, after which they were cover-slipped with Entellan mounting medium (Cat No: 1.07960.0500, Merck Millipore, UK). 


\subsubsection{Standard method of immunohistochemical staining using Ni-DAB}

with targets that require AR with Citrate Buffer.

This protocol is as stated in 2.3.4, with the following additional AR step. Following the second set of washes with 1X PBS and 0.03\% PBS-T, sodium citrate buffer (pH6) was applied to the sections in each well and incubated for 60 minutes antigen retrieval at $70^{\circ} \mathrm{C}$. The excess citrate buffer is removed and protocol continues as described above. 


\section{Chapter 3 Phenotypic Characterisation of the ghrelin receptor (GHS-R) in the adult mouse brain}

\subsection{Introduction}

\subsubsection{Ghrelin Receptor}

Ghrelin mediates its biological effects through its receptor, growth hormone secretagogue receptor (GHSR). This receptor was first discovered by A. D. Howard in 1996, as a protein regulating growth hormone $(\mathrm{GH})$ release (Howard et al., 1996). It is a G-protein coupled receptor consisting of 7 transmembrane domains, GHSR1a, and a truncated version of this receptor consisting of only 5 transmembrane domains, GHSR1b, which has been shown to form heterodimers with GHSR1a and regulate its signalling ability and its ability to form dimers with other GPCR's (Navarro et al., 2016) . Ghrelin undergoes post-translational modification, whereby an octanoyl group is added by ghrelin o-acyl transferase (GOAT) to proghrelin to enable binding and signalling via GHSR (Gutierrez et al., 2008). It has been shown that both forms of ghrelin have the ability to cross the blood-brain barrier (BBB) (Banks, Op, Robinson, \& Heiman, 2002), but it is AG that travels across the BBB and binds to GHSR located in the hippocampus (Diano et al., 2006).

GHSR is expressed in regions of the brain which are important for learning and memory consolidation, one of these being the dentate gyrus (DG) situated in the hippocampus (Guan et al., 1997). With the use of in-situ hybridisation of GHSR mRNA, the DG showed high mRNA levels for the receptor along with many other brain regions, such as the multiple 
hypothalamic nuclei (anteroventral preoptic nucleus, arcuate nucleus, paraventricular nucleus), CA2 and CA3, and the substantia nigra pars compacta in the brain stem.

\subsubsection{GHSR-eGFP transgenic mouse model}

Due to the difficulty in producing antibodies of adequate selectivity and sensitivity against GPCRs, because of cell surface receptors lacking in sufficient antigenicity (Reichenbach, Steyn, Sleeman, \& Andrews, 2012), the GHSR-eGFP mouse model has been used to determine expression of GHSR in the adult mouse brain. It has shown greater sensitivity in mRNA expression of GHSR in extra-hypothalamic regions such as the hippocampus. This reporter mouse model was made by inserting multiple copies of an adapted bacterial artificial chromosome (BAC RP23-358A16) that contains the GHSR gene, into which an enhanced GFP coding sequence was been incorporated into the translational start site of GHSR (Mani et al., 2014).

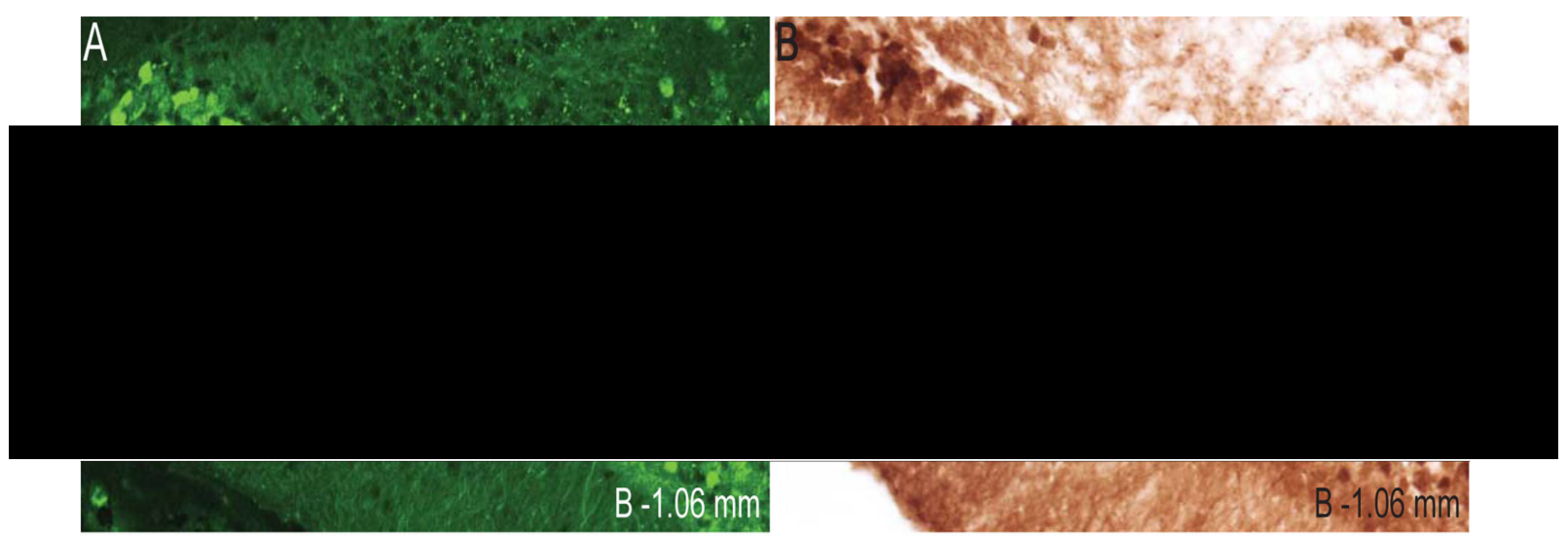

Figure 3.1 Representative images of GHSR-eGFP expression in the granule cell layer of the DG.

Greater endogenous eGFP expression via fluorescence (A) in comparison to eGFP immunoreactivity, showing eGFP+ cells in the DG (B). Image taken from (Mani et al., 2014). 


\subsubsection{Aim of Chapter}

The aim of this chapter is to characterise GHSR1a expression in cells involved in the process of post-developmental hippocampal neurogenesis. In turn, this will aid us in beginning to clarify ghrelin's action in the hippocampus and how it regulates AHN. 


\subsection{Materials \& Methods}

\subsubsection{Mice}

Adult male GHSR-eGFP reporter mice $(n=6)$ were utilised for this study. These mice were generated by the GENSAT (Gene Expression Nervous System Atlas) project at Rockefeller University. These transgenic mice contain several copies of an altered bacterial artificial chromosome that contains the GHSR gene (BAC RP23-358A16), where a sequence coding for enhanced GFP is inserted at the translational start site of GHSR (Mani et al., 2014).

\subsubsection{Tissue Preparation}

Mouse brain samples were collected following fixation, as stated in the general methods 2.1. All procedures were performed with ethical approval at Monash University, Australia. Swansea University's Animal Welfare and Ethical Review Panel (AWERP) approved the studies.

\subsubsection{Tissue Sectioning}

Mouse brain issue was sectioned as stated in the general methods 2.2. Sections were collected specifically into a 12-well Nunclon plate, using a 1:6 ratio. 


\subsubsection{Immunofluorescent staining of Type I NSPC's, proliferating cells and mature granule cells with GHSR-GFP.}

See protocol 2.3.2, with the following amendments.

$5 \%$ NGS in $0.1 \%$ PBS-T was applied to the tissue for 1 hour at RT. The block was subsequently removed and the primary $\left(1^{\circ}\right)$ antibodies Nestin, Ki67, or NeuN along with GFP (see Table 3.1) in 0.1\% PBS-T, were applied in a cocktail (all together) and incubated overnight at $4^{\circ} \mathrm{C}$, on a rocker. Sections were subsequently washed as before in 1x PBS, and the appropriate secondary $\left(2^{\circ}\right)$ antibodies in $0.1 \%$ PBS-T (see Table 3.2) were applied to the tissues and incubated for 30 minutes at RT.

Finally, tissue sections were washed as before in 1x PBS and, mounted and coverslipped as previously stated (2.3.1).

\subsubsection{Immunofluorescent staining of Type II NSPC's with GHSR-GFP.}

See protocol 2.3.2, with the following specifics.

A block of 5\% NGS in 0.1\% PBS-T was applied for 60 minutes at RT. Excess block was removed, the $1^{\circ}$ antibody for Sox 2 was applied in $0.1 \%$ PBS$\mathrm{T}$ (see Table 3.1) and incubated overnight at $4^{\circ} \mathrm{C}$ on a rocker. Subsequently, the appropriate $2^{\circ}$ antibody was applied (see Table 3.2) for 30 minutes at RT. Sections were washed as before in $1 \times$ PBS. The $1^{\circ}$ antibody for GFP in $0.1 \%$ PBS-T was applied for 1 hour at RT. After this incubation, the sections were washed as before, and the appropriate $2^{\circ}$ antibody was applied (see Table 2) for 30 minutes at RT.

Finally, tissue sections were washed as before in 1x PBS and mounted onto glass slides, as previously mentioned above in 2.3.1. 
Table 3.1: List of Primary Antibodies used.

\begin{tabular}{|c|c|c|c|c|}
\hline Target & Cell Type & Species & $\begin{array}{l}\text { Company \& Cat. } \\
\text { No. }\end{array}$ & Dilution \\
\hline GFP & GHSR & Chicken & Abcam (Ab13970) & $1: 1000$ \\
\hline NeuN & $\begin{array}{l}\text { Mature Granule } \\
\text { cells }\end{array}$ & Mouse & Millipore (MAB377) & 1:1000 \\
\hline Sox 2 & Type II NSPCs & Rabbit & Abcam (Ab97959) & $1: 500$ \\
\hline Nestin & Type I NSPCs & Mouse & Abcam (Ab6142) & $1: 1000$ \\
\hline Ki67 & $\begin{array}{l}\text { Proliferating } \\
\text { cells }\end{array}$ & Rabbit & Abcam (Ab16667) & $1: 500$ \\
\hline
\end{tabular}

Table 3.2: List of Fluorescent Secondary Antibodies used.

Secondary $\mathrm{Ab} \quad$ Company \& Cat. No Dilution

\begin{tabular}{|c|c|c|}
\hline Alexa Fluor 488 Goat a-Chicken IgG $(\mathrm{H}+\mathrm{L})$ & $\begin{array}{c}\text { Molecular Probes } \\
\text { (A11039) }\end{array}$ & 1:500 \\
\hline Alexa Fluor 568 Goat a-Mouse IgG $(\mathrm{H}+\mathrm{L})$ & $\begin{array}{c}\text { Molecular Probes } \\
\text { (A11004) }\end{array}$ & 1:500 \\
\hline Alexa Fluor 568 Goat a-Rabbit IgG $(\mathrm{H}+\mathrm{L})$ & $\begin{array}{c}\text { Molecular Probes } \\
\text { (A11011) }\end{array}$ & 1:500 \\
\hline
\end{tabular}

\subsubsection{Analysis of fluorescent immunoreactivity.}

Fluorescently labelled tissue sections (4 sections per mouse, $\mathrm{n}=6$ mice per each analysis) were analysed for their immunoreactivity using a Zeiss Imager M1 microscope with Axiocam MRm. High resolution imaging of colocalised proteins throughout the z-axis was performed using a Laser Scanning Confocal Microscope (LSCM) (Zeiss LSM 710). The brain regions were defined by their Bregma co-ordinates with the use of The Mouse Brain 
Atlas (Paxinos, G., Franklin, 2012). Representative images of the immunoreactivity seen are displayed in the results section. 


\subsection{Results}

\subsubsection{Phenotypic Characterisation of GHSR in the Dentate Gyrus of adult}

mice.

The DG of the adult mammalian hippocampus is a neurogenic niche where new neurones are generated throughout life. AG has been shown to enhance AHN (Kent et al., 2015), we sought to determine whether ghrelin's receptor, GHSR1a, was expressed within this neurogenic niche.

In order to establish general GHSR expression in the adult murine hippocampus and to determine the cell specific expression of this receptor, fluorescent immunohistochemical staining was performed on $30 \mu \mathrm{m}$ thick coronally cut hippocampal sections, were collected from adult GHSR-eGFP reporter mice ( $n=6,4$ sections stained per mouse). Antibodies against type I $\left(\mathrm{Nestin}^{+}\right)$and type II (Sox2 $\left.{ }^{+}\right)$neural stem progenitor cells (NSPCs) were used, along with markers for proliferating cells $\left(\mathrm{Ki} 67^{+}\right)$and mature granule cells $\left(\mathrm{NeuN}^{+}\right)$, to determine co-localisation with GHSR (eGFP+). General extrahypothalamic expression of GHSR was observed in regions associated with learning and memory (Figure 3.2), the hippocampus, cingulate cortex, entorhinal cortex and the basal lateral amygdala. GHSR is strongly expressed in the DG in the GCL cell bodies, with some expression observed in the hilus. Also, axonal projections to the CA3 were immunoreactive for GHSR. As shown in Figure 3.3, no co-localisation was observed between the GHSR$\mathrm{eGFP}^{+}$cells in the DG and type I or type II NSPCs. However, Sox ${ }^{+}$cells were closely associated with GHSR-eGFP+ cells (Figure 3.4), which could suggest a possible paracrine relationship. Similarly, proliferating $\mathrm{Ki}^{6} 7^{+}$cells were not co-localised with GHSR-eGFP+ cells (Figure 3.5). All mice stained showed the same immunoreactive pattern as shown in the representative images. 


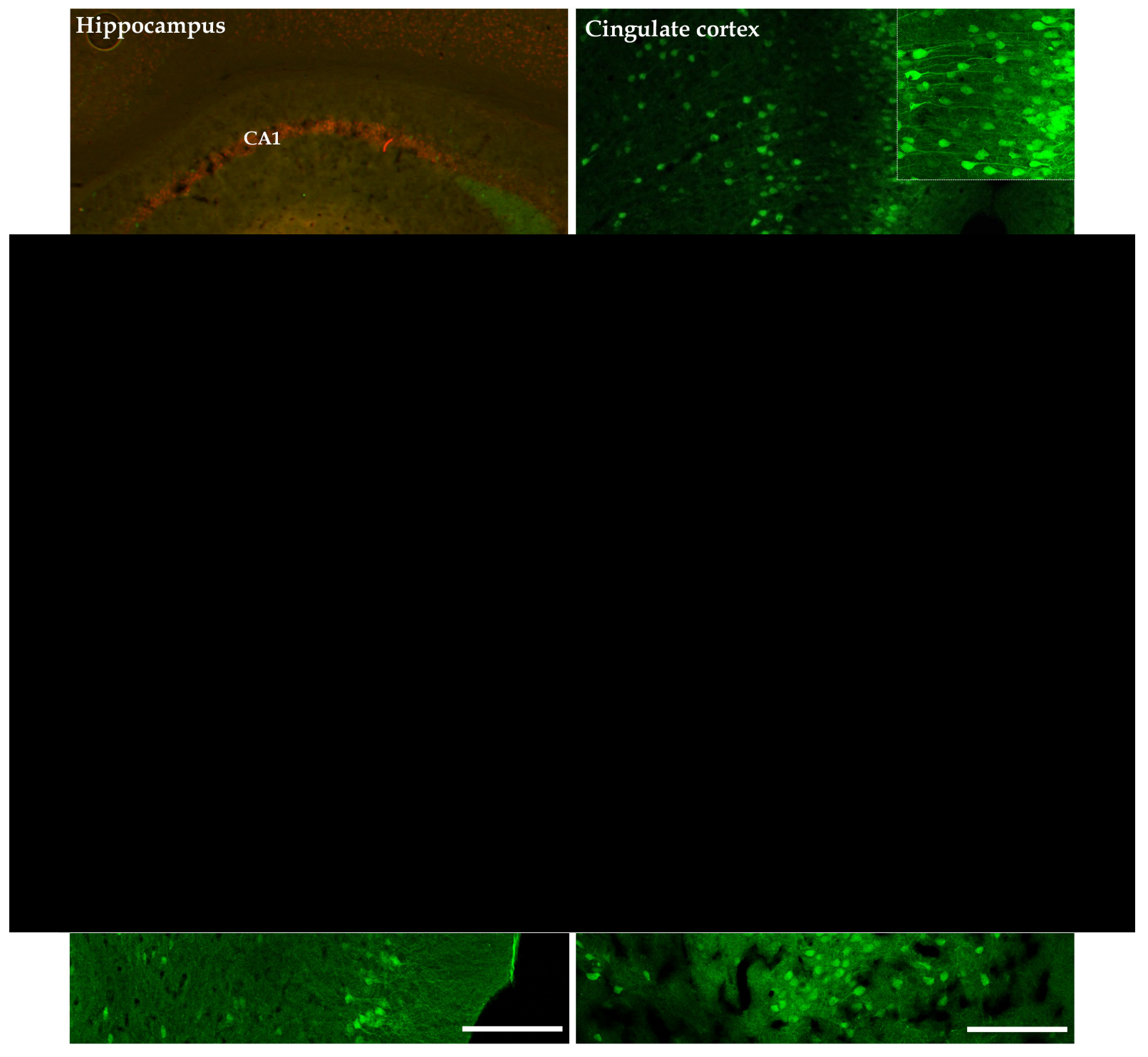

Figure 3.2. General GHSR-eGFP expression in the hippocampus and other areas associated with learning and memory.

Extra-hypothalamic expression of GHSR is confirmed in adult male GHSR-eGFP mice, robust expression in the learning and memory centres; hippocampus, cingulate cortex, entorhinal cortex and the basal lateral amygdala. Scale bar $=50 \mu \mathrm{m}$. Inset images $=40 \mathrm{x}$. 


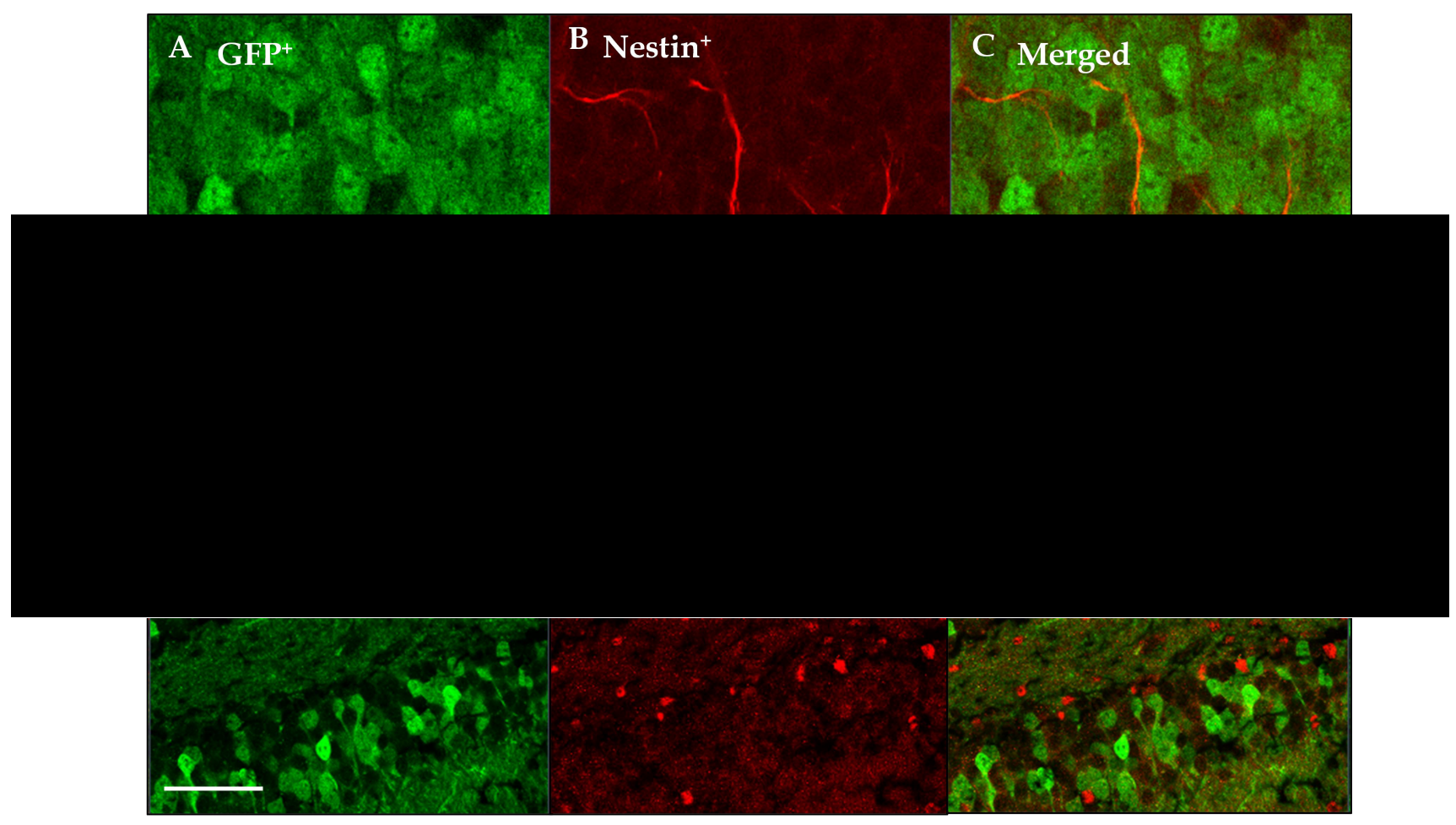

Figure 3.3. GHSR is not expressed on Type I (Nestin+) or Type II (Sox2+) NSPC's.

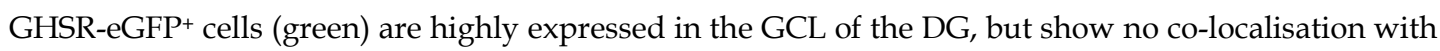
Type I NSPC's (A-C) or with Type II NSPC's (D-F) (red). N =6, 4 slices per mouse. Scale bar $=50 \mu \mathrm{m}$ (Hornsby et al., 2016).

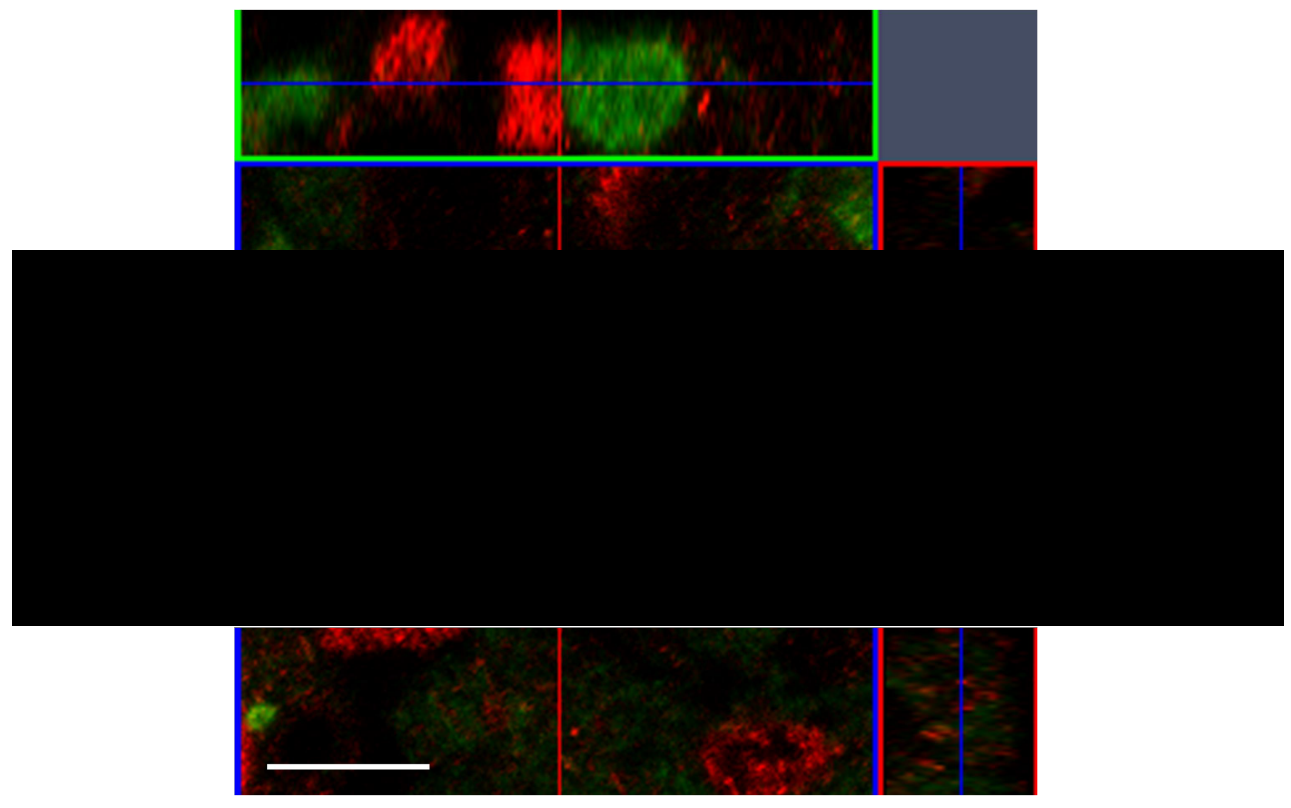

Figure 3.4. Close association of GHSR-GFP+ cells with Type II SOX2+ NSCs suggests a possible paracrine function.

Example orthogonal image showing GHSR-GFP ${ }^{+}$cells (green) with Type II Sox2 ${ }^{+}$NSCs (red) are not co-localised, but have a close proximity to each other. $\mathrm{N}=6,4$ slices per mouse. Scale Bar $=10 \mu \mathrm{m}$ (Hornsby et al., 2016). 


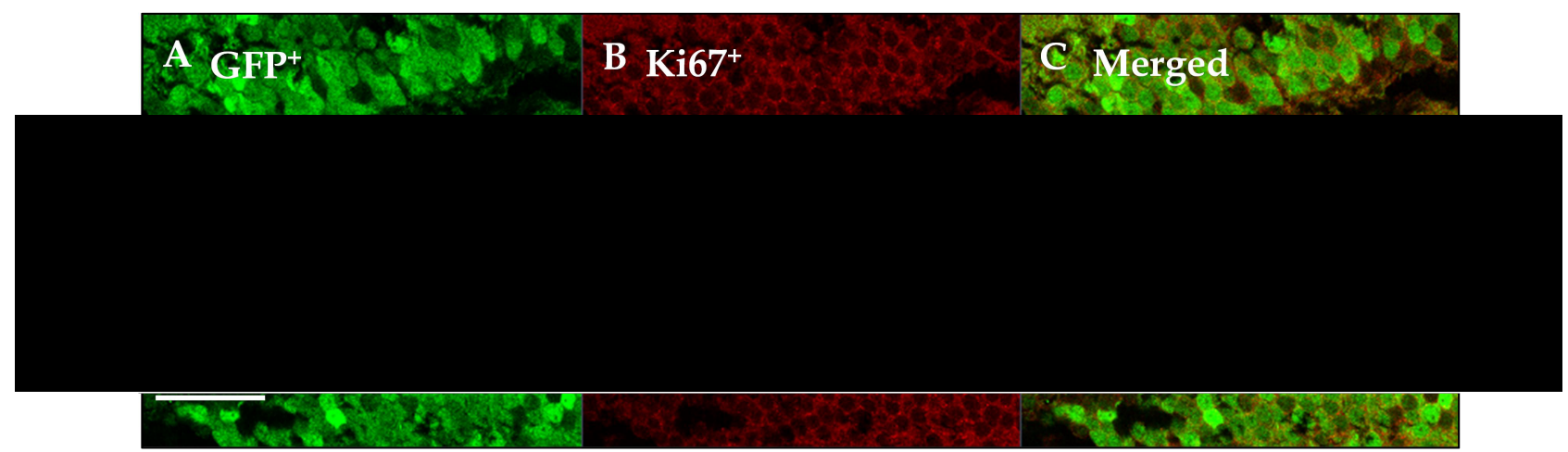

Figure 3.5. Proliferating cells (Ki67) do not express GHSR in the DG.

No co-localisation is shown between GHSR-eGFP+ cells (green) and $\mathrm{Ki}^{+} 7^{+}$expressing proliferating cells (red) (A-C). $\mathrm{N}=6,4$ slices per mouse. Scale bar $=50 \mu \mathrm{m}$ (Hornsby et al., 2016).

These findings suggest that GHSR has no direct effect on NSPCs or dividing cells. In contrast, we observed extensive co-localisation of GHSR$\mathrm{eGFP}^{+}$with the mature granule cell marker, NeuN (Figure 3.6). Indicating that, differentiated neuronal cells within the GCL express GHSR.

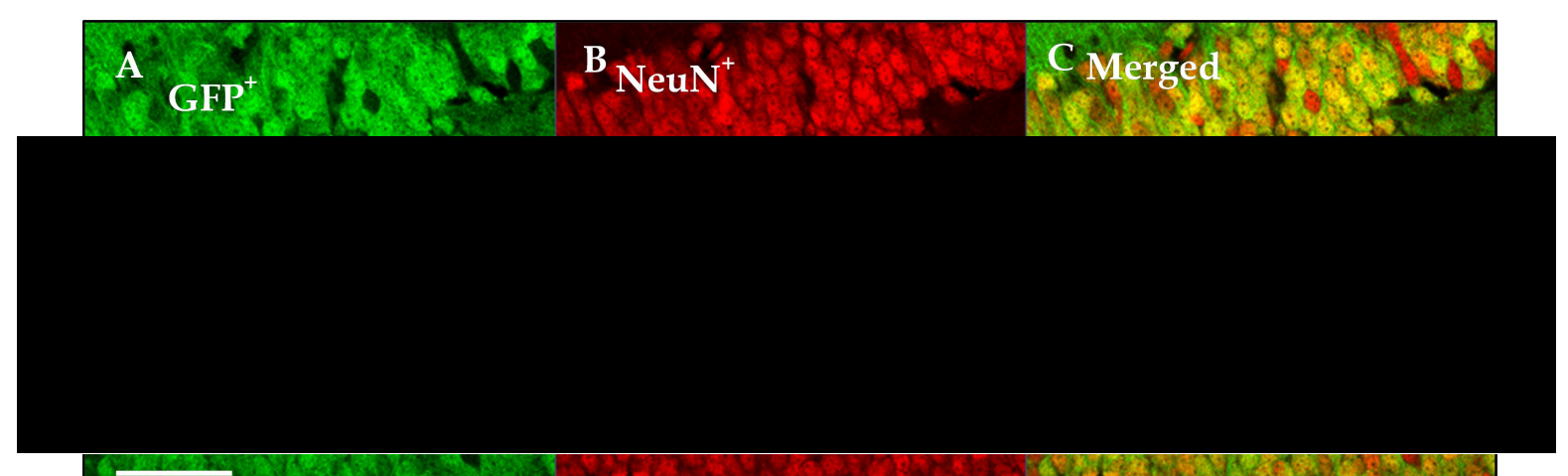

Figure 3.6. Mature Granule cells (NeuN+) express GHSR in the DG.

Extensive co-localisation of GHSR-eGFP+ cells (green), and $\mathrm{NeuN}^{+}$in mature granule cells (red) (A-C) of the DG. Scale bar $=50 \mu \mathrm{m}$ (Hornsby et al., 2016). 


\subsection{Discussion}

The aim of this chapter was to phenotypically characterise the expression of the ghrelin receptor, GHSR, in the neurogenic niche of the hippocampus. The GHSR-eGFP transgenic mouse model was used to give accurate representation of GHSR expression in the DG via eGFP immunoreactivity.

The results from this work firmly indicate that GHSR is expressed predominantly on mature $\mathrm{NeuN}^{+}$granule cells in the GCL of the DG, and that these neurones appear to show close association with type II Sox $2^{+}$ NSPCs, an example of which is shown in Figure 3.4.

We know that the adult brain generates new neurones following embryonic development (Altman \& Das, 1965), and that this continuous qualitative process occurs in specific neurogenic niches, the SGZ of the DG of the hippocampus and the SVZ of the lateral ventricles (Gonzallez-Perez, 2012). These new adult born neurones functionally integrate into the existing neural circuits (Aimone et al., 2014) and contribute to normal brain function, and aid in learning and memory (Deng, Aimone, \& Gage, 2010). AHN has been shown to be important for the encoding of pattern separation memory, the ability to distinguish between contexts that have slight changes or the ability to utilise memories obtained from stimuli that are similar in time and/or space. A study in adult rats where AHN was removed by $\mathrm{x}$ irradiation of the hippocampus, showed that these rats performed worse in pattern separation tasks where stimuli were presented with low spatial separation, compared to controls (Clelland et al., 2009).

The main cell type produced in this unique microenvironment, are mature granule cells $\left(\mathrm{NeuN}^{+}\right)$. These cells functionally integrate by sending axonal projections into the CA3 region where they form excitatory synaptic boutons (Kempermann et al., 2015). My work using GHSR-eGFP reporter mice shows that ghrelin's receptor, GHSR, is predominately expressed on 
these mature $\mathrm{NeuN}^{+}$granule cells in the DG. The expression observed with this immunofluorescent staining is in line with that demonstrated by Mani et al. 2014. Identical GHSR expression was shown in the hippocampus, cingulate cortex, lateral entorhinal cortex and the basal lateral amygdala in the GHSR-eGFP mice used in this study, compared to Mani et al. 2014.

Notably, the receptor was not expressed on either radial glial-like type I Nestin $^{+}$or non-radial glial-like type II Sox $2^{+}$NSPCs located in the DG (Figure 3.3), which are cells key to the expansion phase neurogenesis. These type I NSPC's are quiescent progenitors with long radial-glial like projections, that become activated in response to physiological activity such as exercise, give rise to type II NSPC's (Ladran, Tran, Topol, \& Brennand, 2013; Lugert et al., 2010). Type II NSPC's are multipotent and give rise to immature neurones, granule cells, glial and astrocytic cells, as well as more type II NSPC's. They divide asymmetrically as to not deplete the stem cell pool and are self-renewing (Suh et al., 2007). However, Sox $2^{+}$NSPCs and $\mathrm{GHSR}^{+}$cells, presumably neurones, were in close proximity within the SGZ. These findings indicate that whilst ghrelin-GHSR signalling may not directly regulate NSPCs there may be indirect modulation via soluble paracrine factors that promote neurogenesis, and add that an extra control over the modulation of AHN.

With this in mind, it is understood that ghrelin does mediate paracrine effects, by increasing intracellular Ca2+ signalling between neurones and glial cells (Avau et al., 2013). Ghrelin could possibly have a similar effect on pro-neurogenic molecules such as brain-derived neurotrophic factor (BDNF) within the DG. BDNF is highly expressed in the hippocampus and is important for neuronal plasticity (Beck \& Pourié, 2013) and promoting neurogenesis (Rossi et al., 2006). BDNF in the DG has also been shown to be needed for the consolidation of pattern separation memory, as it stimulates immature neurones (Bekinschtein et al., 2013, 2014). Also, intrinsic factors such as Wnt proteins, and both its canonical and non-canonical signalling, 
play an important role in $\mathrm{AHN}$ regulation, from the determination of granule cell fate to the control of maturation of neuroblasts (Schafer et al., 2015).

Fibroblast growth factor 2 (FGF-2) is another soluble factor that could communicate between $\mathrm{GHSR}^{+}$neurones and Sox2 ${ }^{+}$NSPCs. It has been shown to be important for the maturation of progenitor cells in the hippocampus (neurogenesis), as demonstrated by FGF-deficient mice. These mice had no change in the number of proliferating cells compared to WT, but showed a specific decrease in the number of new-born mature neuronal cells in the DG, suggesting that FGF-2 is important for the process of differentiation into mature neuronal cells (Werner, Unsicker, \& von Bohlen und Halbach, 2011). This was work done following on from previous findings by Yoshimura et al. 2001, who showed that endogenous FGF-2 was essential for neuro-progenitor proliferation and differentiation in the adult DG, following brain injury in mice.

Ghrelin-GHSR induced AHN could also be induced via another mechanism. Physical activity has been shown to promote AHN in male Sprague-Dawley rats. Rats that had been selectively bred to be high responders to activity (HRT) and low responders (LRT) underwent prolonged aerobic exercise by voluntary wheel running, endurance running on a treadmill or high intensity interval training (HIIT). The HRT mice that had undergone wheel running and endurance training showed the greatest increase in $\mathrm{DCX}^{+}$cells in the hippocampus compared to sedentary controls, after 6-8 weeks of training (Nokia et al., 2016).

To summarize, GHSR is expressed in regions of the brain that are important for learning and memory, and is specifically expressed in mature dentate granule neurones in the GCL of the DG, but shows no expression on type I and II NSPC's or proliferating cells. This suggests that circulating AG has the ability to cross the BBB and stimulate AHN via its receptor GHSR present in the neurogenic niche of the DG, and facilitate this in a cell-extrinsic manner. 


\section{Chapter 4 The direct and indirect effects of ghrelin on adult hippocampal plasticity}

\subsection{Introduction}

Calorie restriction (CR) or a low-calorie diet has been associated with the amelioration of learning and memory. CR is an evolutionary conserved process that occurs in various species and reduces the incidence of disease and promotes longevity (Fontana et al., 2010). It has been shown in mouse models of ageing and neurodegenerative disease that restricting diet has a beneficial effect; protecting against the loss of hippocampal, cortical and striatal neurones (W Duan et al., 2001). This has also led to a reduction in spatial learning and memory deficits and age-associated physiological impairments in humans such as insulin sensitivity and glucose handling (Witte et al., 2009). Longitudinal studies in adult rhesus monkeys (7-14 years old, 46 males and 30 females) have shown that CR (30\% less calories to controls) can reduce the incidence of age-related deaths when the regime is introduced at an early age or later in adult life. In this study they measured age-related and all-cause mortality, which were both improved by $C R$, as was the incidence of age-related disorders like diabetes, neoplasia and sarcopenia (Colman et al., 2014). In light of this, it can be hypothesised that the introduction of a CR diet to adults suffering from age-related diseases, such as Parkinson's disease and Alzheimer's could benefit from it (Gräff et al., 2013). Studies have looked at CR as a way to delay or prevent the onset of such disorders, shown in rhesus monkeys that had undergone 6 months of $30 \% \mathrm{CR}$ then treated with a neurotoxin (MPTP) to induce hemiparkinson condition. They showed elevated DA levels and better locomotor activity compared to ad lib controls (Maswood et al., 2004), and it has shown to reduce amyloid- $\beta$ peptides in squirrel monkeys when CR was implemented 
in early adulthood, lessening AD amyloidosis (Qin et al., 2006). The effects of CR have not been investigated once the disorder has been established.

$\mathrm{CR}$ is thought to mediate its beneficial effects, in part, by promoting hippocampal plasticity and neurogenesis, and one possible mediator is thought to be ghrelin (Zane B Andrews, 2011). CR was initially shown to increase the number of new-born cells in the DG of rats (3 month old males, ad lib $n=24,30 \% C R n=24$, for 3 months). Rats were treated with the thymidine analogue bromodeoxyuridine (BrdU, 50mg/kg) daily for 5 days, and the CR group showed a significant increase in BrdU' cells in the DG of the hippocampus (J Lee, Duan, Long, Ingram, \& Mattson, 2000). Following this, a study demonstrated that the presence of ghrelin was required for CR to have its pro-neurogenic effect, with the use of ghrelin $\mathrm{KO}$ mice. This was performed by CR in WT and ghrelin KO mice (24 hour fast followed by 24 hour fed regime) for 3 months, with ad libitum fed controls, which were also administered BrdU (50mg/kg, i.p.) daily for 12 days. It was observed that only within the WT CR group was there an increase in the survival of new adult-born cells in the DG (Kim et al., 2015). The orexigenic peptide hormone ghrelin is known to bind to its receptor GHSR, expressed in the hippocampus (Diano et al., 2006) and ghrelin increases following CR (Lutter et al., 2008). It has also been shown to promote cell proliferation in the hippocampus (Johansson et al., 2008; Moon et al., 2009).

\subsubsection{Aim of this Chapter}

The aim of this work is to determine the mechanisms by which ghrelin mediates its neurogenic effect with the use of ghrelin and GHSR knockout mouse models, and whether manipulating ghrelin levels directly via AG injection and/or indirectly by CR will facilitate improvements in AHN and learning and memory in mice. 


\subsection{Materials \& Methods}

\subsubsection{Mice and Experiments}

\subsubsection{GHSR-eGFP mice.}

Adult male GHSR-eGFP reporter mice were used in this study, as described in 2.2.1. This 4-day experiment was designed to determine the effects of direct and indirect elevations of AG on neurogenesis. AG levels were increased directly by injection $(1 \mathrm{mg} / \mathrm{kg}$ i.p) 1 hour before the end of the fast, indirectly by calorie restriction (CR) (16 hour overnight fast) or a combination of the two, in order to quantify IEG expression following shortterm exposure to CR and/or AG. There were 4 groups ( $n=4$ per group); wild type (WT), CR, AG and CR+AG. Mice were terminally anesthetised with sodium pentobarbital, transcardially perfused with $0.9 \%$ solution of $\mathrm{NaCl}$, followed by perfusion with $4 \%$ paraformaldehyde (PFA) solution. The brains were then immersed in 4\% PFA overnight and placed in 30\% sucrose solution for cryoprotection until sunk.

\subsubsection{GHSR knock out mice.}

12 week old adult male and female GHSR knockout (KO) mice (homozygous loxP TB-flanked KO) (Zigman et al., 2005) and WT littermates were individually housed under normal laboratory condition for 7 days before the start of the experiment (12 hours light, 12 hours dark), so that animals acclimatised to the conditions and that ad-libitum (AL) levels of feeding could be monitored for each genotype. Mice were separated into 4 groups; AL fed WT, AL fed GHSR KO, CR WT and CR GHSR KO (6 male and 6 female per 
group, $\mathrm{n}=12$ per group). $\mathrm{CR}$ was set at $70 \%$ of the total calories of the AL fed group, and the $\mathrm{CR}$ mice were exposed for the first 14 days of the experiment. Mice were administered the thymidine analogue BrdU (50mg/kg) (B5002, Sigma Aldrich) on days 4-7 to label newly dividing cells. Following the 14 days of $\mathrm{CR}$, mice were able to feed AL for the rest of the experiment. On days 31-44 the mice underwent behavioural testing; contextual fear condition (CFC), for assessment of hippocampal dependant memory, which was performed by Dr Katia Amstalden, Texas A\&M University (see below 4.2.1.4 and Figure 4.1). Full schematic is shown in Figure 4.5.

These mice were gifted from Prof. Jeffery Zigman of the University of Texas Southwestern (Dallas, Texas, USA), and were from crosses between mice that were heterozygous for the GHSR KO allele and C57/BL6 animals for $>10$ generations, to prevent producing recombinant inbreeds. All animal experiments were performed at Texas A\&M University.

\subsubsection{Ghrelin Knockout mice.}

Female C57/BL6 WT and Ghrelin KO littermate mice, ranging from 5 to 16 months of age underwent a 28-day experiment. On experimental days 4 to 7 mice were administered once-daily injections of BrdU, 50mg/kg i.p (B5002, Sigma Aldrich) dissolved in saline $(0.9 \% \mathrm{NaCl}, \mathrm{pH} 7)$. On day 27 the mice were either fasted overnight (during the dark phase) or not; lights off $6 \mathrm{pm}-$ 6am with the standard laboratory chow removed. Approximately half of the CR group were refed for 1 hour during the light phase (10am-11am), resulting in 6 different groups. On day 28 the mice underwent terminal anaesthesia with isofluorane, following which they were decapitated, the trunk blood collected and the brain tissue removed and bisected. One hemisphere was sectioned for immunohistochemical analysis, and the other was frozen at $-80^{\circ} \mathrm{C}$ for future protein/RNA/DNA extraction. Full schematic is shown in Figure 4.9. 
These Ghrelin KO mice were a gift from our collaborator Prof. Yuxiang Sun of Baylor College of Medicine, that had been back crossed into a homozygoes C57/BL6 genetic background >/= to 10 generations.

\subsubsection{Behavioural Testing: Contextual Fear Conditioning}

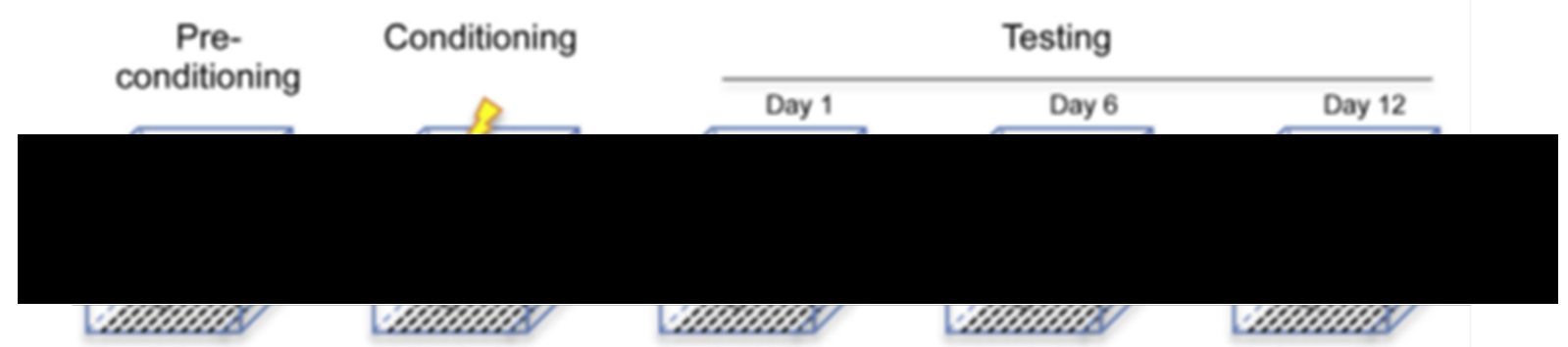

Figure 4.1 Diagram depicting CFC behavioural test.

Graphic illustrating the different stages of the CFC paradigm (Hornsby et al. 2015).

Contextual fear conditioning (CFC) assesses hippocampal-dependant memory formation and function, with the test used specifically looking at context-dependant fear memory by measuring freezing behaviour following foot shock ((Kent et al., 2015; van Woerden et al., 2007). AHN has been shown to be important for enhancing this type of context-dependant fear memory (Kitamura et al., 2009). WT and GHSR KO mice underwent CFC in the following way; for 30 minutes a day, 6 days before the conditioning, mice were moved to a test room, where the apparatus was cleaned with 70\% $\mathrm{EtOH}$ before introducing the animals to the chamber. The animals were preintroduced to a non-aversive environment $(25 \mathrm{~cm} \times 25 \mathrm{~cm}$ Coulbourn Habitest chamber with wire grid floor) for 7.5 minutes. 2 days later mice were introduced to the same conditioning chamber that was distinct due to the addition of a coloured wall panel, for 2.5 minutes before being given a 2 second foot-shock (0.5 mA). 2.5 minutes later another 2 second foot-shock was given, before being returned to its home cage. CFC was then measured by observing freezing behaviour in the absence of a foot-shock, when mice 
were placed back into the conditioning chamber for 2.5 minutes 1, 6 and 12 days after the initial conditioning (Figure 4.1). During this period, the presence and absence of freezing was recorded via camcorder placed directly above the cage. A trained person, blind to the genotype of the mice, scored freezing behaviour every 5 seconds over the 2.5 minute period.

\subsubsection{Tissue Preparation}

GHSR-eGFP mouse brain samples were collected and fixed as stated in the general methods 2.1, until required for analysis. All procedures were performed with ethical approval at Monash University, Australia. Swansea University's Animal Welfare and Ethical Review Panel (AWERP) approved the studies.

C57/BL6 and Ghrelin KO mouse brain samples underwent immersion fixation in $4 \%$ PFA overnight at $4^{\circ} \mathrm{C}$. Subsequently, they were cryoprotected in $30 \%$ sucrose solution until they had sunk, after which they were stored in PBS with $0.1 \%$ Sodium Azide at $4^{\circ} \mathrm{C}$ until required for analysis.

\subsubsection{Tissue Sectioning}

Mouse brain tissue was sectioned as stated in the general methods 2.2. Sections were collected specifically into 24-well Nunclon plates, in a ratio of $1: 12$.

4.2.4 Immunoflurescent staining of immediate early gene (IEG) activation in GHSR-eGFP mice.

For co-localisation of eGFP with the IEG's, c-Fos and Egr-1, protocol 2.3.2 was used with the following amendments. 
Antigen retrieval was performed using citrate buffer $(\mathrm{pH} 6)$ for 20 minutes at $90^{\circ} \mathrm{C}$, then blocked with 5\% NGS as previously stated. GFP antibody (see Table 4.1) was applied and incubated at $4^{\circ} \mathrm{C}$ overnight. Following secondary antibody application and incubation (Table 4.2) and washes, c-Fos or Egr-1 primary antibody diluted in 0.1\% PBS-T (1:2000 or 1:1000 respectively) were applied for overnight incubation at RT on a rocker. Following another wash of the sections, they were incubated in biotinylated secondary antibody diluted in $0.1 \%$ PBS-T for 60 minutes at RT. Sections were washed and then incubated for 30 minutes at RT with streptavidin AlexaFluor594 conjugate diluted in $0.1 \%$ PBS-T. Finally, sections were washed, with the addition of the nuclear counterstain, Hoescht $(1: 10,000)$, to the first wash. Sections were then mounted as described above. 
Table 4.1 Primary antibodies used.

Target Cell Type Species Company \& Cat. Dilution No.

\begin{tabular}{|c|c|c|c|c|}
\hline GFP & GHSR & Chicken & Abcam (Ab13970) & 1:1000 \\
\hline c-Fos & IEG & Rabbit & Santa Cruz (Sc-52) & $1: 1000 / 1: 2000$ \\
\hline Egr-1 & IEG & Rabbit & Santa Cruz (Sc-189) & $1: 1000$ \\
\hline BrdU & $\begin{array}{l}\text { New adult-born } \\
\text { Cells }\end{array}$ & Rat & $\begin{array}{l}\text { ABD Serotec } \\
(\text { MCA2060) }\end{array}$ & $\begin{array}{l}\text { 1:400 IF } \\
\text { 1:3000 Ni-DAB }\end{array}$ \\
\hline NeuN & $\begin{array}{l}\text { Mature Granule } \\
\text { Cells }\end{array}$ & Mouse & Millipore (MAB377) & 1:1000/1:2000 \\
\hline Sox2 & Type II NSPCs & Goat & $\begin{array}{l}\text { Santa Cruz (Sc- } \\
17320)\end{array}$ & 1:500 IF \\
\hline Sox2 & Type II NSPCs & Rabbit & Abcam (Ab97959) & $1: 1000 \mathrm{Ni}-\mathrm{DAB}$ \\
\hline Nestin & Type I NSPCs & Mouse & Abcam (Ab6142) & $1: 1000$ \\
\hline Ki67 & $\begin{array}{l}\text { Proliferating } \\
\text { Cells }\end{array}$ & Rabbit & Abcam (Ab16667) & $1: 500$ \\
\hline DCX & $\begin{array}{l}\text { Immature } \\
\text { neurones }\end{array}$ & Goat & Santa Cruz (Sc-8066) & $1: 500$ \\
\hline
\end{tabular}


Table 4.2 Secondary antibodies and conjugated used.

\section{Secondary $\mathbf{A b}$}

\begin{tabular}{|c|c|c|}
\hline Alexa Fluor 488 Goat a-Chicken IgG (H+L) & $\begin{array}{l}\text { Molecular Probes } \\
\text { (A11039) }\end{array}$ & 1:500 \\
\hline Alexa Fluor 568 Goat a-Mouse IgG $(\mathrm{H}+\mathrm{L})$ & $\begin{array}{l}\text { Molecular Probes } \\
\text { (A11004) }\end{array}$ & $1: 500$ \\
\hline Alexa Fluor 568 Goat a-Rabbit IgG $(\mathrm{H}+\mathrm{L})$ & $\begin{array}{l}\text { Molecular } \\
\text { (A11011) }\end{array}$ & $1: 500$ \\
\hline Alexa Fluor 488 Goat a-Rabbit IgG $(\mathrm{H}+\mathrm{L})$ & $\begin{array}{l}\text { Molecular Probes } \\
\text { (A11034) }\end{array}$ & 1:500 \\
\hline $\begin{array}{l}\text { Alexa Fluor } 488 \text { Donkey a-Rabbit IgG } \\
(\mathrm{H}+\mathrm{L})\end{array}$ & $\begin{array}{l}\text { Molecular } \\
\text { (A21206) }\end{array}$ & 1:500 \\
\hline Alexa Fluor 568 Donkey a-Goat IgG $(\mathrm{H}+\mathrm{L})$ & $\begin{array}{l}\text { Molecular Probes } \\
\text { (A11057) }\end{array}$ & $1: 500$ \\
\hline Alexa Fluor 488 Goat a-Rat IgG $(\mathrm{H}+\mathrm{L})$ & $\begin{array}{l}\text { Molecular Probes } \\
\text { (A11006) }\end{array}$ & $1: 500$ \\
\hline Biotinylated Goat a-Rabbit IgG & $\begin{array}{l}\text { Vector Laboratories } \\
\text { (BA-1000) }\end{array}$ & 1:400 \\
\hline Biotinylated Goat a-Mouse IgG & $\begin{array}{l}\text { Vector Laboratories } \\
\text { (BA-9200) }\end{array}$ & $1: 400$ \\
\hline Biotinylated Goat a-Rat IgG & $\begin{array}{l}\text { Vector Laboratories } \\
\text { (BA-9400) }\end{array}$ & $1: 400$ \\
\hline $\begin{array}{l}\text { Biotin-SP-conjugated AffiniPure Donkey a- } \\
\text { Goat } \operatorname{IgG}(\mathrm{H}+\mathrm{L})\end{array}$ & $\begin{array}{l}\text { Jackson Laboratories } \\
\text { (705-065-003) }\end{array}$ & $1: 400$ \\
\hline Streptavidin Alexa Fluor 594 conjugate & Invitrogen (S11227) & $1: 500$ \\
\hline
\end{tabular}




\subsubsection{Immunofluorescent staining of proliferating NSPC's, in GHSR- eGFP mice.}

For co-localisation of Ki67 with the NSPC markers Nestin or Sox2, protocol 2.3.2 was used with the following amendments.

Following AR, sections undergoing staining of Ki-67-Nestin were blocked in 5\% NDS, whereas Ki67-Sox2 sections were blocked in 5\% NGS, before incubation with Ki67 primary antibody incubation. Sections were washed as before in PBS and then incubated with fluorescent secondary antibody (see Table 4.2) for 30 minutes at RT. Subsequently, sections were incubated with primary antibody anti-Sox2 diluted in 0.1\% PBS-T at RT for 60 minutes or anti-Nestin primary antibody for 2 hours (Table 4.1). Sections were washed before 30 minute incubation at RT in appropriate secondary antibody (see Table 4.2) diluted in 0.1\% PBS-T. After the final wash, sections were mounted as previously described.

\subsubsection{Immunofluorescent staining of new-born neuronal cells (BrdU- NeuN), in GHSR $\%$ mice and WT littermates.}

See protocol 2.3.3, with the following changes.

Tissues were incubated for 60 minutes at RT in a 5\% NGS block in $0.1 \%$ PBS-T. After the removal of the block solution, the primary antibody antiBrdU diluted in 0.1\% PBS-T (see Table 4.1) was applied. The plate was sealed and placed on a rocker overnight at $4^{\circ} \mathrm{C}$. The primary anti-NeuN antibody was applied diluted in 0.1\% PBS-T (see Table 4.1) and incubated for 60 minutes at RT. Sections were washed as previously described, and the secondary antibody Biotinylated Goat anti-Mouse diluted in 0.1\% PBS-T (see Table 4.2) was added to the sections for 60 minutes at RT. Excess secondary antibody was removed and the sections washed in 1 X PBS as before, 
Streptavidin AlexaFluor 594 diluted in 0.1\% PBS-T (see Table 4.2) was then applied and incubated for 30 minutes at RT.

\subsubsection{Immunohistochemical staining for Type II NSPC's $\left(\mathrm{Sox}^{+}\right)$using Nickel enhanced DAB (di-aminobenzidine) (Ni-DAB).}

This was performed using the method stated in 2.3.5, with the following specifics.

Following AR and blocking in 5\% NGS, the primary antibody anti-Sox2 (Table 4.1) was applied to the sections and incubated overnight at $4^{\circ} \mathrm{C}$ in the cold room, on a rocker.

\subsubsection{Immunohistochemical staining for immature neuronal cells (DCX) using Ni-DAB.}

This staining was performed as stated in the method 2.3.4, with the following specifics.

Tissue sections were blocked in 5\% NDS block in 0.03\% PBS-T and incubated for 60 minutes at RT. Sections were incubated in primary antibody DCX was then applied to the sections, diluted in 0.03\% PBS-T and 2\% NDS, this was left to incubate overnight at $4^{\circ} \mathrm{C}$ in the cold room, on a rocker.

\subsubsection{Immunohistochemical staining for new-born cells (BrdU) using Ni-} DAB.

Mouse brain sections were washed in 2 times in 1X PBS, 10 minutes each followed by a wash on $0.03 \%$ PBS-T for 10 minutes. Sections were then incubated in 1 X PBS with $1.5 \% \mathrm{H}_{2} \mathrm{O}_{2}$ for 20 minutes at RT. Sections were 
washed again in 1X PBS and 0.03\% PBS-T as before, after which they were incubated in $2 \mathrm{M} \mathrm{HCl}$ at $37^{\circ} \mathrm{C}$ for 30 minutes followed by a wash in Borate Buffer for 10 minutes. Following a wash series the sections were immersed in 5\% NGS block for 60 minutes at RT. BrdU primary antibody (see Table 4.1) diluted in $0.03 \%$ PBS-T and 2\% NGS was applied and the sections incubated overnight at $4^{\circ} \mathrm{C}$ in the cold-room, on a rocker. Sections were washed as before and secondary biotinylated antibody diluted in $0.03 \%$ PBS-T and 2\% NDS, was applied to the sections and incubated for 70 minutes at RT and also covered from light. Following another wash, a premixed ABC solution was applied $(0.4 \% \mathrm{~A}+0.4 \% \mathrm{~B}$ in $0.03 \%$ PBS-T, premixed for 30 minutes before application); this was incubated for 90 minutes at RT. Subsequently, sections for were washed twice in 1X PBS for 10 minutes each, and the washed in sodium acetate $(\mathrm{pH})$ for 10 minutes. Tissues were reacted in Ni-DAB and glucose oxidase solution for between 5-10 minutes. Following this reaction the sections were washed 2 times in 1X PBS.

The sections were then mounted into glass slides as described previously, and allowed to dry overnight. The slides were subjected to a counterstain process with methyl green. Methyl green was pre-heated at $60^{\circ} \mathrm{C}$ for at least 30 minutes prior to staining. The slides were rinsed with tap water, $\sim 30$ seconds per slide. The slides were then placed in the pre-heated methyl green for 5-10 minutes at $60^{\circ} \mathrm{C}$. Following this, the slides were washed in deionised $\mathrm{H}_{2} \mathrm{O}\left(\mathrm{ddH}_{2} \mathrm{O}\right)$ until clear. In a fume hood, the slides were dipped into acetone 5-10 times, followed by an alcohol dehydrations series consisting of dipping the slides for 30 seconds in 2x 95\% EtOH and 2x 100\% EtOH and 1x Histoclear. The slides were coverslipped as before. 


\subsubsection{Immunofluorescent staining of new-born neuronal cells (BrdU/NeuN), and new-born neuronally active cells (BrdU/c-Fos), in WT and Ghrelin KO mice.}

Mouse brain sections were washed 3 times in 1X PBS for 5 minutes each following which there was permeabilised in cold methanol and placed at $20^{\circ} \mathrm{C}$ for 2 minutes. Sections were washed again, as above, and incubated in $2 \mathrm{M} \mathrm{HCl}$ for 30 minutes at $37^{\circ} \mathrm{C}$. After a 10 -minute wash in borate buffer, the sections were washed again in PBS after which a 5\% NGS + 5\% BSA (bovine serum albumin) (A7906-10KG, Sigma Aldrich) block diluted in 0.1\% PBS-T was added and incubated at RT for 60 minutes. Upon removal of excess block the BrdU primary antibody diluted in 0.1\% PBS-T was applied and left to incubate for 24 hours at RT. Following a wash series, the sections were incubated with fluorescent secondary antibody (see Table 4.2) for 30 minutes at RT, and protected from light. Following washes in PBS the tissue was incubated in primary antibodies for either c-Fos or NeuN (diluted in $0.1 \%$ PBS-T) were applied to the sections and incubated for 24 hours at RT, after which they were washed as before and the incubated in the appropriate biotinylated secondary antibodies diluted in 0.1\% PBS-T (see Table 4.2) were applied and sections incubated for 60 minutes in the dark at RT. Sections were subsequently washed in PBS and ABC solution was applied $(0.4 \% \mathrm{~A}$ $+0.4 \% \mathrm{~B}$ in $0.1 \%$ PBS-T, premixed for 30 minutes before application) for 90 minutes at RT. Following another set of washes in PBS, the tyramine signal amplification (TSA) system was used (bTyr 1:100 $+\mathrm{H}_{2} \mathrm{O}_{2}$ 1:1000 in 0.1\% PBS$\mathrm{T}$ ), this solution was applied to the sections for 10 minutes at RT, after which they were washed. Streptavidin conjugate diluted in $0.1 \%$ PBS-T was then applied to the sections for 30 minutes at RT. Finally, sections were washed in PBS and then mounted into glass slides with Prolong Gold mounting medium (P36930, Life Technologies) and stored as described before. 
4.2.11 Immunofluorescent staining of new-born neuronal cells (BrdUNeuN), in WT and Ghrelin KO mice.

See protocol 4.2.6, with the following changes.

After incubation of NeuN $1^{\circ}$ primary antibody, sections were washed in 1X PBS as previously stated, followed by incubation in secondary antibody (Goat anti-Mouse AlexaFluor 568, see Table 4.2) was applied and the sections incubated for 30 minutes at RT. Sections were washed a final time as before, mounted onto glass slides and cover-slipped as previously stated.

\subsubsection{Analysis of fluorescent immunoreactivity.}

Fluorescently labelled tissue sections were analysed for their immunoreactivity using a Zeiss Imager M1 microscope with Axiocam MRm. High resolution imaging of co-localised proteins throughout the z-axis was performed using a Laser Scanning Confocal Microscope (LSCM) (Zeiss LSM $710)$.

\subsubsection{Analysis of Ni-DAB immunoreactivity.}

Ni-DAB immunolabelled sections were analysed for their immunoreactivity using the Nikon Eclipse 50i microscope system and the NIS Elements F4 software. Cells were either counted manually where possible or with the use of ImageJ software. 


\subsubsection{Data and Statistical Analysis.}

\subsubsection{IEG activation in GHSR-eGFP mice.}

For this analysis, $\mathrm{n}=4$ per group and 4 sections were chosen from each mouse (2 rostral and 2 caudal coronal sections, each section containing 2 DGs) to undergo staining and fluorescent analysis. The immuno-labelled cells (Egr1-GFP and c-Fos-GFP) were counted manually using the 40x objective and scrolling through the z-axis of the whole GCL of the DG, CingC, BLA and LEnt. Area measurements of the DG were obtained from Hoescht counterstaining and measured using ImageJ software. The resulting cell counts by area were achieved by dividing average cell count by average area measurement. Statistical analysis was performed using Graphpad Prism 7.0 software, using a One-way ANOVA with Fishers LSD post-hoc comparison test.

\subsubsection{Cell proliferation in the DG of GHSR-eGFP mice.}

For this analysis, $n=4$ per group and 3 sections were chosen from each mouse (6 DGs in total) to undergo immunofluorescent staining (Ki67-Nestin and Ki67-Sox2) and analysis. The positive cells were counted as stated above (4.2.14.1). Percentages were calculated by dividing the number of double positive cells by the total of Ki67 positive cells and multiplying by 100 .

\subsubsection{Effect of CR on AHN in the adult DG of GHSR KO mice.}

For the analysis of BrdU-NeuN staining in WT and GHSR KO mice; $\mathrm{n}=$ 12 per group, whole well (1:12 ratio) sections with 10-16 sections per well, underwent immunofluorescent staining. Positive cells were counted in the GCL of the DG as described above (4.2.14.1). DGs were separated using the Mouse Brain Atlas (Paxinos, G., Franklin, 2012) into rostral and caudal sections. Average cell counts were multiplied by 360 (1:12 series, each section $360 \mu \mathrm{m}$ apart) to achieve number of cells per DG. Statistical analysis of the data was performed with Graphpad Prism 7, and due to there being 2 
variable factors a 2-way ANOVA was performed with Tukey's post hoc multiple comparisons test.

\subsubsection{Effect of genotype and CR on Type II NSPC number in WT and GHSR KO mice.}

For this analysis, $n=8$ (genotype) or $n=4$ (feeding) per group and 4 sections (from each mouse chosen to undergo immunohistochemical Ni-DAB staining for Sox2. Images were collected as described above (4.2.13) and cell counts in the SGZ of the DG were obtained using ImageJ software. Average cell counts for each mouse were calculated by adding together each sections cell count and dividing by the number of sections. To obtain average cells per DG, average cell count is multiplied by 360 as described before (4.2.14.3). A Mann-Whitney test was performed for statistical analysis on the effect of genotype, comparing 2 groups. To assess the effect of CR (4 groups) a 2-way ANOVA followed by Tukey's post hoc multiple comparisons test was performed.

\subsubsection{Effect of Ghrelin KO on basal neurogenesis levels.}

For the analysis of immunohistochemical staining of type II NSPCs (Sox2), proliferating cells (Ki67) and immature neurones (DCX) in the SGZ of the DG, $\mathrm{n}=4-8$ per group (WT and Ghrelin $\mathrm{KO}$ ). ImageJ was used to draw around the GCL and produce cell counts. Immunofluorescent staining for new-born neurones (BrdU-NeuN), $\mathrm{n}=9-12$ per group. Cell counts were obtained by manual counting (4.2.12). Data was handled as above (4.2.14.4). A 2-tailed student $t$-test was performed with Graphpad Prism.

\subsubsection{Effect of CR on new-born neuronal cell activation in WT and Ghrelin-KO.}

For the analysis of BrdU-c-Fos immunofluorescent staining, $\mathrm{n}=6$ per group and $\sim 4$ sections per mouse. Cell counts and data statistical analysis was handled as mentioned before (4.2.14.3). 
4.2.14.7 Effect of age on new-born neurone and cell activation.

For the analysis of BrdU-c-Fos and BrdU-NeuN immunofluorescent staining $n=4-6$ per group with 4 sections per mouse stained. Cell counts and data statistical analysis was handled as mentioned before (4.2.14.3). 


\subsection{Results}

\subsubsection{The effects of acutely elevating AG levels directly and indirectly in}

the mouse brain.

$\mathrm{CR}$ and $\mathrm{AG}$ have been known to cause improvements in cognitive function and AHN (J.-H. Park et al., 2013; Witte et al., 2009). For this experiment we sought to determine whether increasing levels of circulating AG by $C R$ and/or AG injection would have any effect on hippocampal plasticity and neuronal cell activation in the key brain centres for learning and memory. The areas that we examined were; the DG, the cingulate cortex (CingC), the lateral entorhinal cortex (LEnt) and the basolateral amygdala (BLA), all areas shown to be important for learning and memory.

\subsubsection{CR and AG treatment enhance the expression of the IEG Egr-1 in the DG.}

To determine whether CR or AG induced the expression of IEG's, Egr1 and c-Fos, in DG specific neurones in areas of the brain that are important for learning and memory, AG levels were elevated directly by injection and indirectly by $\mathrm{CR}$, or a combination of the two in GHSR-eGFP mice. Following overnight CR, the expression of Egr-1 was increased in the DG (Figure 4.2B, $\mathrm{P}<0.05$ ), CingC (Figure 4.2F, $\mathrm{P}<0.05$ ) and BLA (Figure 4.2H, $\mathrm{P}<0.05$ ). AG injection had a similar effect in elevating Egr-1 expression in the DG (Figure 4.2B, $\mathrm{P}<0.05$ ), CingC (Figure 4.2F, $\mathrm{P}<0.01$ ) and BLA (Figure 4.2H, $\mathrm{P}<0.05$ ). The combination treatment of $\mathrm{CR}$ and AG injection also increased Egr-1 expression in the DG (Figure 4.2B, $\mathrm{P}<0.01$ ), LEnt (Figure 4.2D, $\mathrm{P}<0.05$ ), CingC (Figure 4.2F, $\mathrm{P}<0.05$ ) and the BLA (Figure 4.2H, P<0.05). There was an increase in the number of double positive (GHSR-eGFP+ $\mathrm{Egr}-1^{+}$) cells in the CingC following CR, AG injection and the combined treatment (Figure 4.2F, 
$\mathrm{P}<0.05 ; \mathrm{P}<0.01 ; \mathrm{P}<0.001$ respectively). A similar observation was made in the BLA via combined treatment of $\mathrm{CR}$ and $\mathrm{AG}$ injection (Figure $4.2 \mathrm{H}, \mathrm{P}<0.05$ ).

However, the same pattern was not observed with c-Fos. c-Fos expression was shown to decrease in the LEnt (Figure 4.3L, P<0.01) and BLA (Figure 4.3P, $\mathrm{P}<0.05$ ) following AG injection, and CR resulted in a decrease in expression in the LEnt (Figure 4.3L, P<0.01). However, an increase in c-Fos expression was seen in both GHSR-eGFP- and GHSR-eGFP+ cells of the DG (Figure 4.3J, $\mathrm{P}<0.05 ; \mathrm{P}<0.01$ ), following AG injection, and an increase in cFos $^{+}$cells in the LEnt (Figure 4.3L, P<0.05), due to the combined treatment of CR and AG injection. Also, an increase in c-Fos ${ }^{+}$cells was shown in the CingC (Figure 4.3N, $\mathrm{P}<0.01$ ) following AG injection.

The Egr-1 work was performed by another member of the Davies Group, Yushi Redhead. I assisted in the supervision of this student - helping to optimise the protocols used, assisting with the imaging, cell counting and data analysis. 


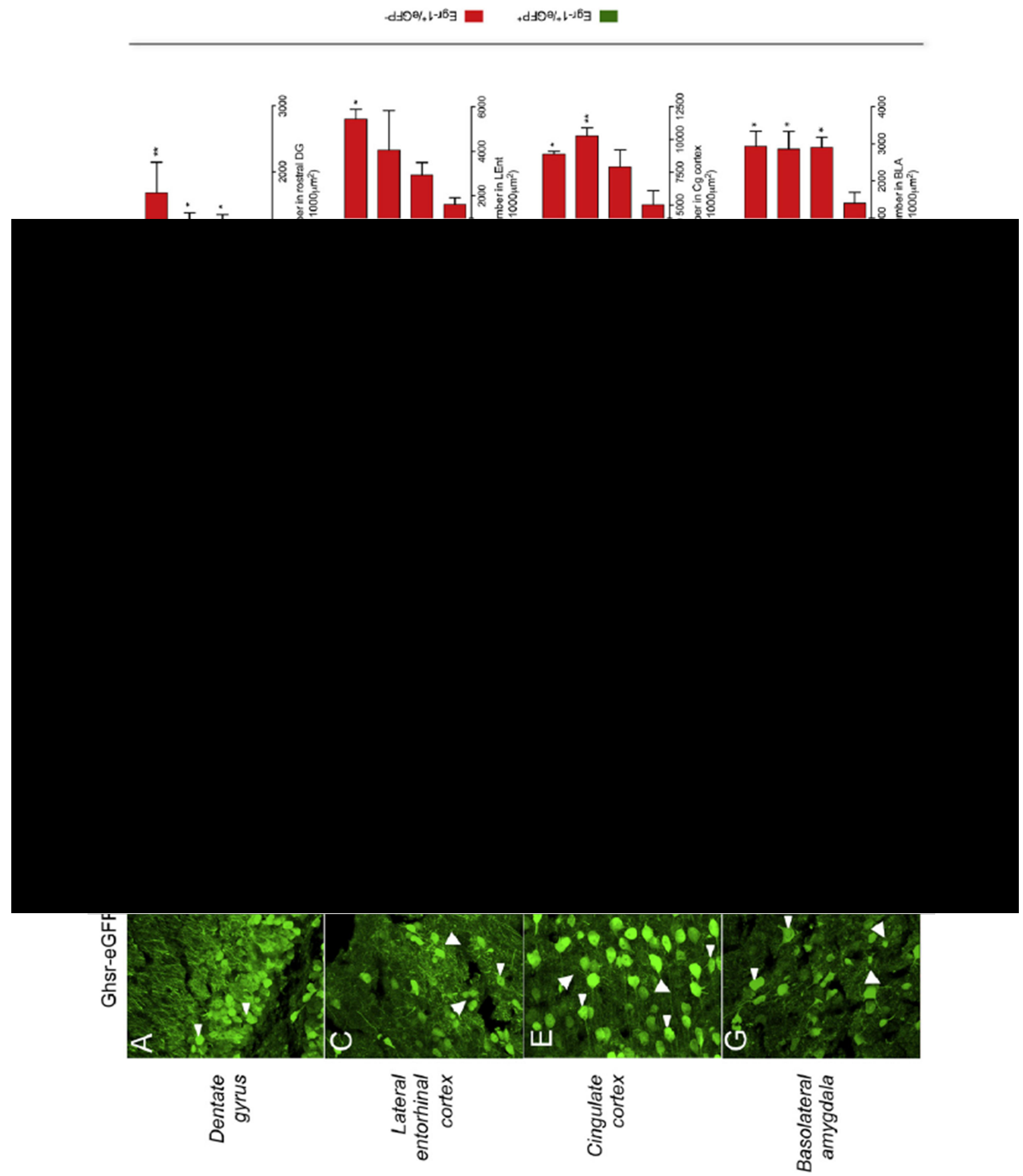

Figure 4.2 CR and AG enhance Egr-1 expression in areas of the brain important for learning and memory.

Example images of Egr-1+ $(\mathrm{A}, \mathrm{C}, \mathrm{E}, \mathrm{G})$ after CR, AG or CR+AG in GHSR-eGFP mice. Increase in Egr-1+ cells in the DG (B), LEnt (D), CingC (F) and BLA (H). Scale bar $=40 \mu \mathrm{m}$. For statistical comparison a One-way ANOVA with Fishers LSD post-hoc analysis performed, ${ }^{*} P<0.05,{ }^{* *} P<0.01,{ }^{* * *} P<0.001$ vs control. All data shown as mean \pm SEM. $n=4$ mice per group. Image taken from Hornsby et al., 2016. 

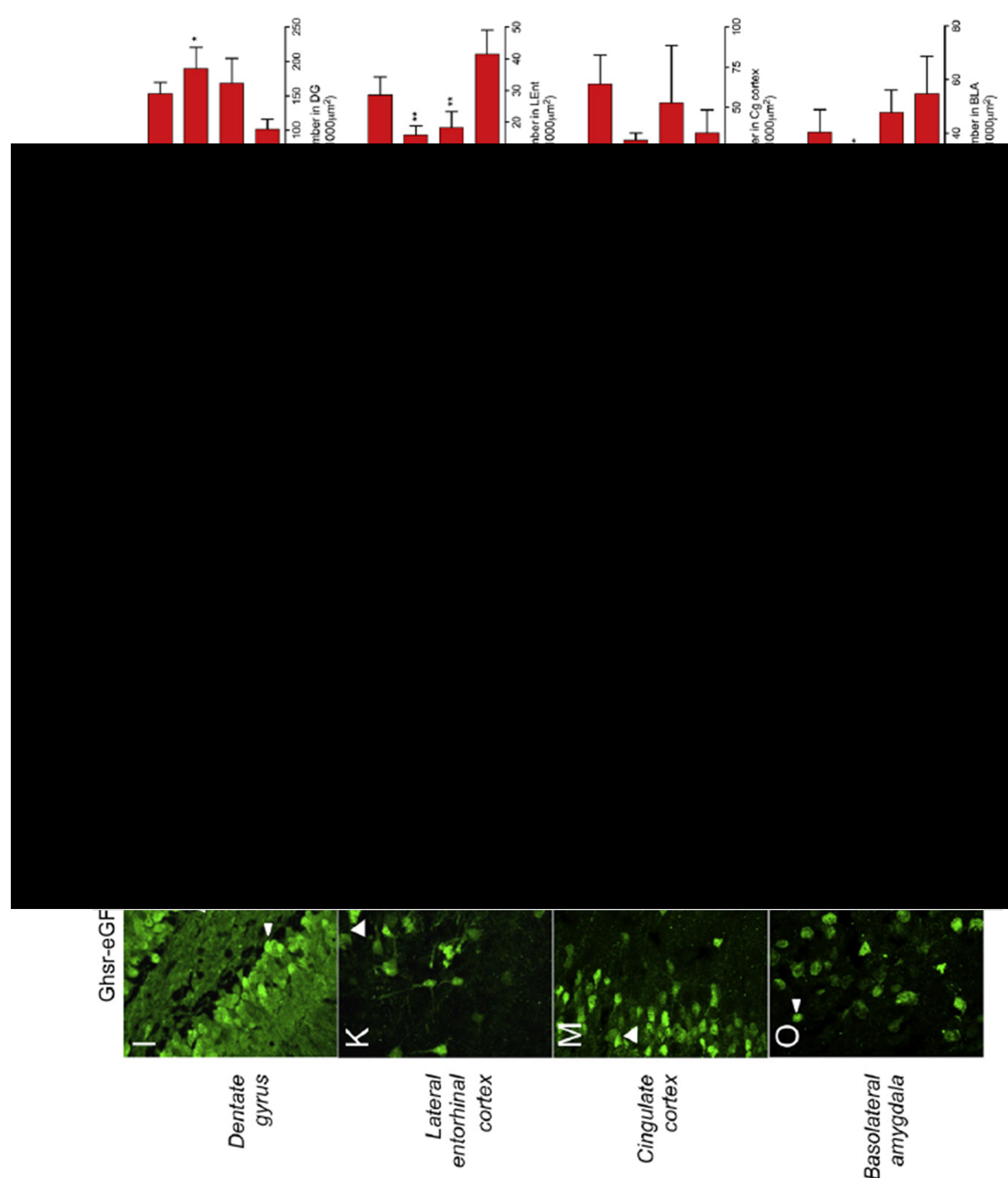

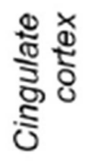

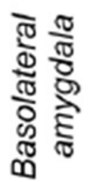

Figure 4.3 CR and AG enhance Egr-1 expression in areas of the brain important for learning and memory, continued.

Example images of c-Fos ${ }^{+}$cells $(\mathrm{I}, \mathrm{K}, \mathrm{M}, \mathrm{O})$ after CR, AG or CR+AG in GHSR-eGFP mice. Cell counts of

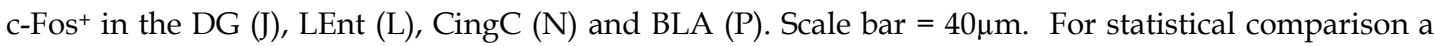
One-way ANOVA with Fishers LSD post-hoc analysis performed, ${ }^{*} P<0.05,{ }^{* *} P<0.01,{ }^{* * *} P<0.001$ vs control. All data shown as mean \pm SEM. $n=4$ mice per group. Image taken from Hornsby et al., 2016. 
4.3.1.2 CR and AG had no effect on cell proliferation or the type of NSPC'S produced.

In order to identify the effects on DG plasticity by indirectly elevating AG levels via $C R$ and directly by i.p. injection of $A G$, or a combination of both $(\mathrm{CR}+\mathrm{AG})$, immunofluorescent staining for proliferating $\mathrm{Ki}^{+}{ }^{+}$cells along with markers for either type I $\left(\mathrm{Nestin}^{+}\right)$or type II (Sox $\left.2^{+}\right)$NSPCs was performed. This was done to determine whether there was any differential effect on the NSPC pool. $30 \mu \mathrm{m}$ thick coronally cut hippocampal sections were collected from adult GHSR-eGFP mice from each treatment group $(n=4)$ and immunofluorescently stained for either Ki67 and Nestin, or Ki67 and Sox2. All $\mathrm{Ki} 67^{+}$cells and double positive cells $\left(\mathrm{Ki}^{6} 7^{+} \mathrm{Nestin}^{+}, \mathrm{Ki}^{+} 7^{+} \mathrm{Sox} 2^{+}\right.$) were manually counted through the z-axis and through the entire rostrocaudal extent of the brain. As can be seen in Figure 4.4, no significant effect was observed on overall rates of cell proliferation in the DG (Figure 4.4E), and there was no apparent effect on the proportion of type I and II NSPC's that were being produced (Figure 4.4C, D). 


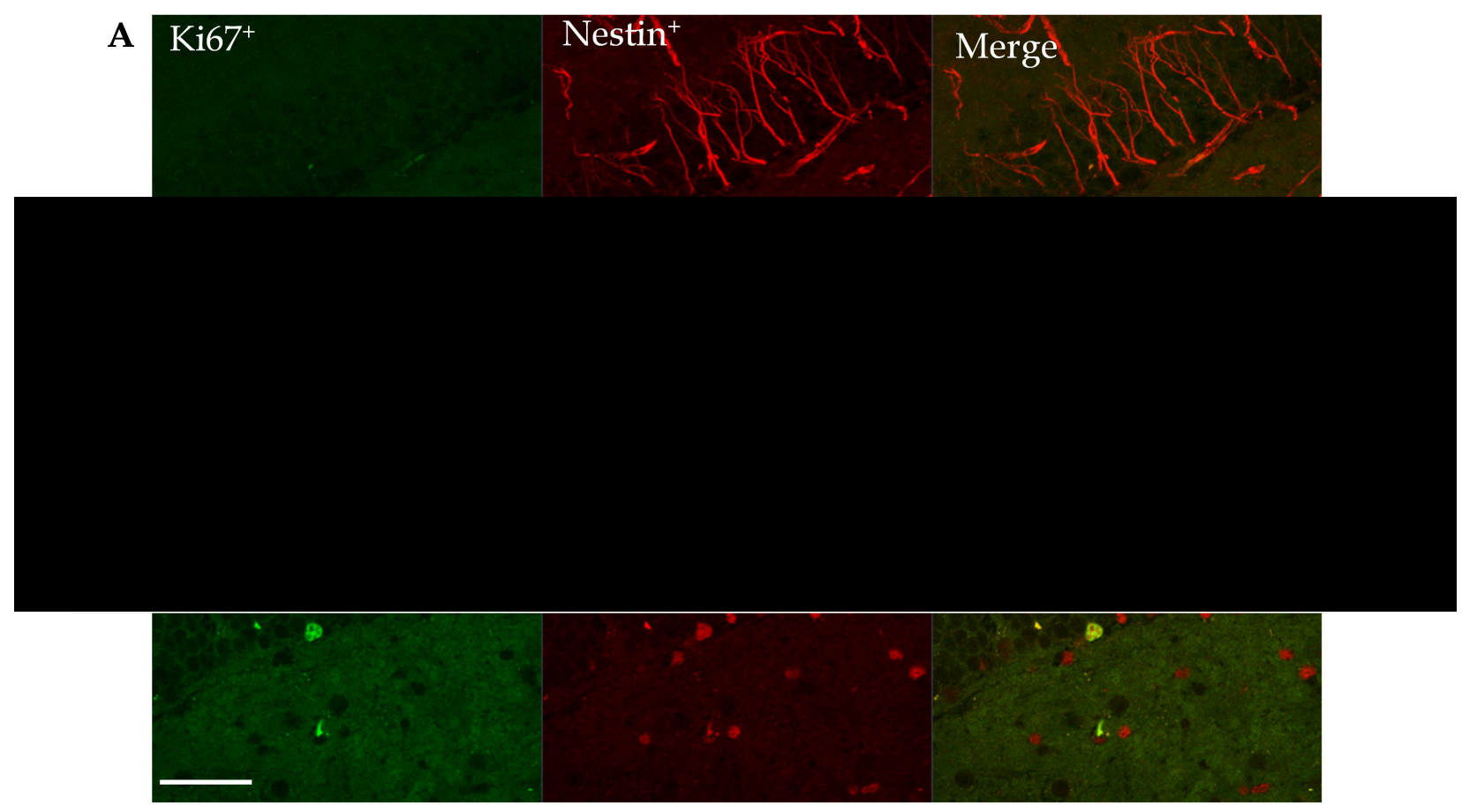

C

D

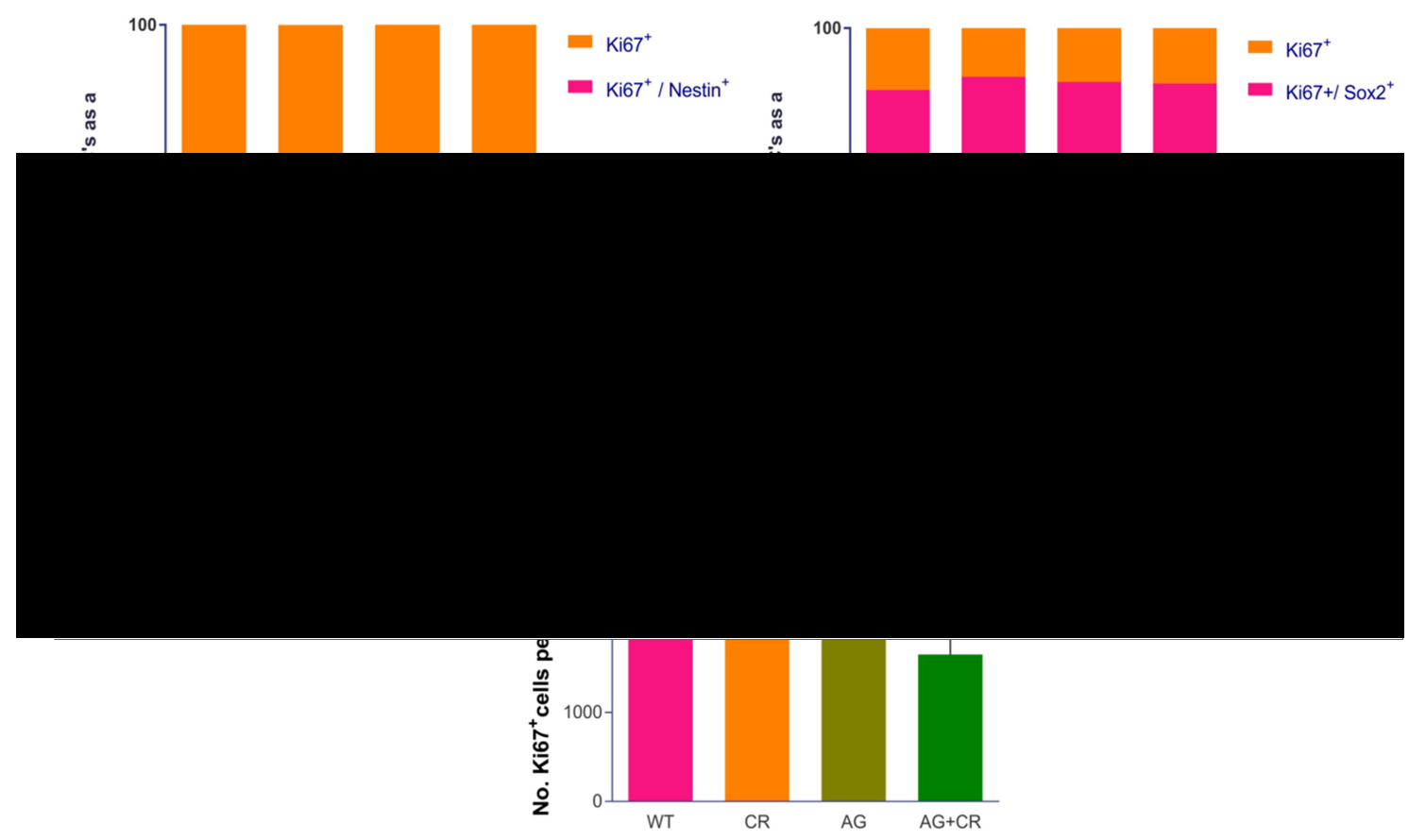

Figure 4.4 Acyl-ghrelin injection and/or CR has no effect on cell proliferation in the DG.

Representative confocal images of double immunofluorescent staining for proliferating (Ki67+) cells with type I radial $\left(\mathrm{Nestin}^{+}\right)$and type II non-radial $\left(\mathrm{Sox}^{+}\right)$cells $(\mathbf{A}, \mathbf{B})$. Scalebar $=50 \mu \mathrm{m}$. CR and AG treatment or the CR/AG combined showed no effect on the proliferation rates of $\mathrm{Nestin}^{+}$and $\mathrm{Sox}^{+}$ cells $(C, D)$ in the mouse hippocampus, and there was no effect on the total number of differentiating cells in the DG (E) $(P>0.05)$. Data represented as mean \pm SEM. 


\subsubsection{Acute CR enhances AHN and context dependant fear memory in a GHSR-dependant manner.}

Overnight CR increased DG Egr-1 expression, as this is important for functionally integrating new-born cells into the adult DG (Veyrac et al., 2013), we analysed the effect of a 2 week period of CR on GHSR KO mice and their WT littermates. This experimental design allowed us to determine the effects of CR on the generation of new-born mature neurones in the adult DG, and whether this was dependent upon GHSR.

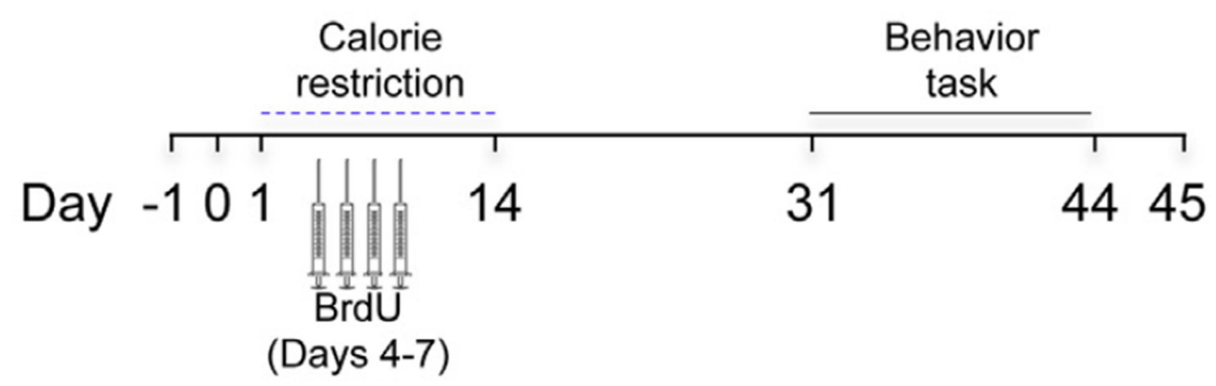

Figure 4.5. Schematic of GHSR KO mice experimental time-line.

Experimental paradigm of 2 week CR in WT and GHSR KO mice. BrdU (50mg/kg i.p. injection) was administered on days $4-7$, behavioural testing on days 31-45, and mice terminally anaesthetized on day 45.

Immunofluorescent staining was performed on $30 \mu \mathrm{m}$ thick coronal mouse brain sections that were collected 31 days after the final day of CR (Day 45) (Figure 4.5), for the quantification of the number of new adult-born mature neuronal cells (BrdU' ${ }^{+} \mathrm{NeuN}^{+}$, see Figure 4.6). There was a $52 \%$ increase in the number of adult-born new neurones $\left(\mathrm{BrdU}^{+} \mathrm{NeuN}^{+}\right)$in the rostral portion of the DG of WT CR mice compared to WT ad libitum (ad lib) fed mice. A 2-way ANOVA revealed a main effect of treatment $(\mathrm{F}(1,44)=$ 5.806, $\mathrm{P}=0.0202$ ) and a post-hoc Tukey multiple comparison test showed a significant difference between the WT CR and WT ad lib mice $(\mathrm{P}=0.048)$ (Figure 4.6B). No effect was seen in the GHSR KO mice. Also, CR was shown to increase the fraction of new-born $\mathrm{BrdU}^{+}$cells that became mature $\mathrm{NeuN}^{+}$ neuronal cells in WT mice (Figure 4.6C, $\mathrm{P}=0.0108$ ), but not in GHSR KO mice $(P>0.99)$. Finally, there was no observed difference in the total number 
of new born cells for either treatment or genotype (Figure 4.6D, P > 0.05). In addition to this data there was no difference in the number of $\mathrm{BrdU}^{+} \mathrm{NeuN}^{+}$ cells in the DG of WT ad lib mice and their GHSR KO counterparts $(\mathrm{P}=$ 0.9812), suggesting that basal neurogenesis isn't impaired in GHSR KO mice. These data indicate that GHSR is required for CR's positive effects on AHN to occur.

The same experimental WT and GHSR KO mice also underwent memory testing 17 days following the final day of $\mathrm{CR}$, on day 31. The contextual fear conditioning (CFC) test was used to determine the effects of $\mathrm{CR}$ on hippocampal-dependent fear memory and is reported to be sensitive to changes in AHN (Kitamura et al., 2009). There was no difference between the GHSR KO and WT littermate mice during training in the "shock" part of the test, indicating that both groups could similarly attain and retain fear memory (2-way ANOVA, genotype $(\mathrm{F}(1,44)=0.8882, \mathrm{P}=0.3511)$; treatment $(F(1,44)=0.009506, P=0.9228))$, shown in Figure 4.6E. Conversely, with the progression of time the CR WT mice displayed an improved retention of their fear memory, retaining it 12 days following their exposure to the fear condition, in comparison to the WT ad libitum (ad lib) fed group. Statistical analysis showed a significant interaction between genotype and treatment (2way RM ANOVA, F $(6,88)=2.971, P=0.0109)$. Post-hoc multiple comparison showed a significant difference between WT CR and WT ad lib littermates (Tukey test, $\mathrm{P}=0.0121$ ) 12 days post-conditioning, but no significant difference was observed between the GHSR KO mice in either group $(\mathrm{P}=0.1837)$ (Figure 4.6E). Also observed was the gradual decline of fear memory in the WT ad lib over the 12 days, which reached statistical significance (Day 1 post-conditioning vs Day 12 post-conditioning in WT/adlibitum mice, $\mathrm{P}=0.0179$ ). The same was not seen in the ad lib fed GHSR KO mice (Day 1 post-conditioning vs Day 12 post-conditioning in GHSR KO / ad-libitum fed mice, $\mathrm{P}=0.1889$ ), implying that GHSR signalling is required in this memory retention extinction process. 
This animal work was performed by Dr Katia Amstalden of Texas A\&M University, Texas, USA, before the brains were shipped to Swansea University for analysis.

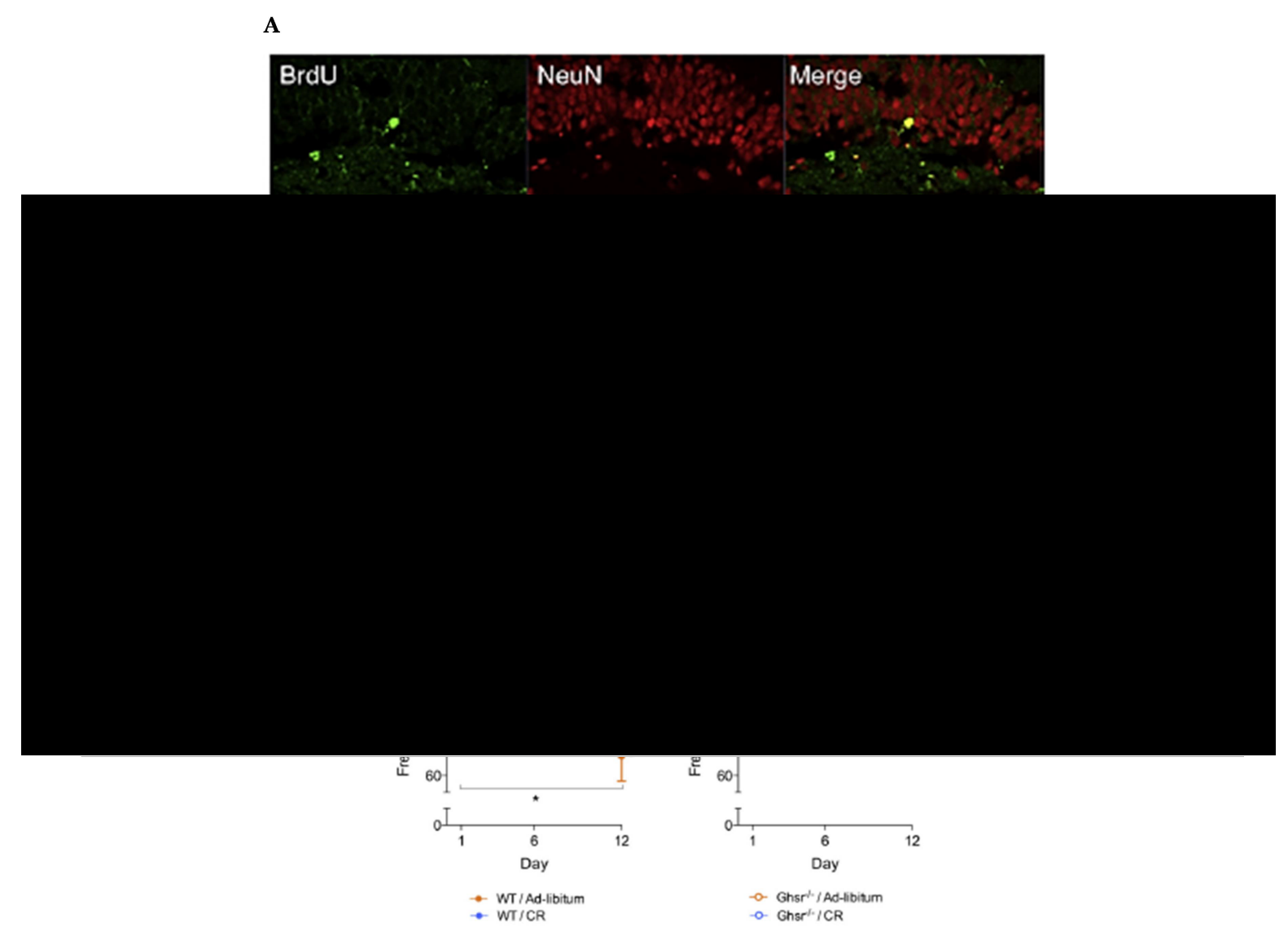

Figure 4.6 A 2 week period of CR enhances AHN in the adult DG and improves context dependant fear memory.

Representative image of $\mathrm{BrdU}^{+}$(red) and $\mathrm{NeuN}^{+}$(green) cells in the DG. Scale bar $=50 \mu \mathrm{m}(\mathrm{A})$. New adult born mature neuron cell number (B); neuronal differentiation rate in newly divided NSPCs (C) and number of new-born cells (D) in the rostral DG. $\mathrm{n}=12$ per group. 2-way ANOVA and Tukey's post hoc test: ${ }^{*} \mathrm{p}<0.05$. Freezing in the absence of foot shock quantification. $\mathrm{n}=12$ per group. 2 -way RM-ANOVA/Tukey's: * $p 0.05$ (E). All data shown are mean \pm SEM Image taken from Hornsby et al. 2016. 
4.3.2.1 Acute CR has no effect on the Type II NSPC pool in the DG of WT and GHSR-KO mice.

The WT and GHSR KO mice were also stained for type II NSPC marker Sox2, to determine the effect of genotype and/or CR on the stem cell pool. Sections were stained using Ni-DAB immunochemical protocol $(n=4$ per group) (Figure 4.7). Sox $2+$ cells were then counted using ImageJ and analysed in Graphpad Prism 7. Data analysis revealed no significant difference in Sox2+ cell number between GHSR-KO and WT littermates (Mann Whitney test, $\mathrm{P}=0.7209$; Figure 4.7B), indicating that the genetic $\mathrm{KO}$ has not adversely affected the stem cell niche. However, a significant increase in Sox $2^{+}$cells was reported in GHSR-KO CR compared to GHSR-KO ad lib fed mice (Tukeys's multiple comparison test following 2-way ANOVA, P = 0.0338, F(1, 12) = 8.799; Figure 4.7C).

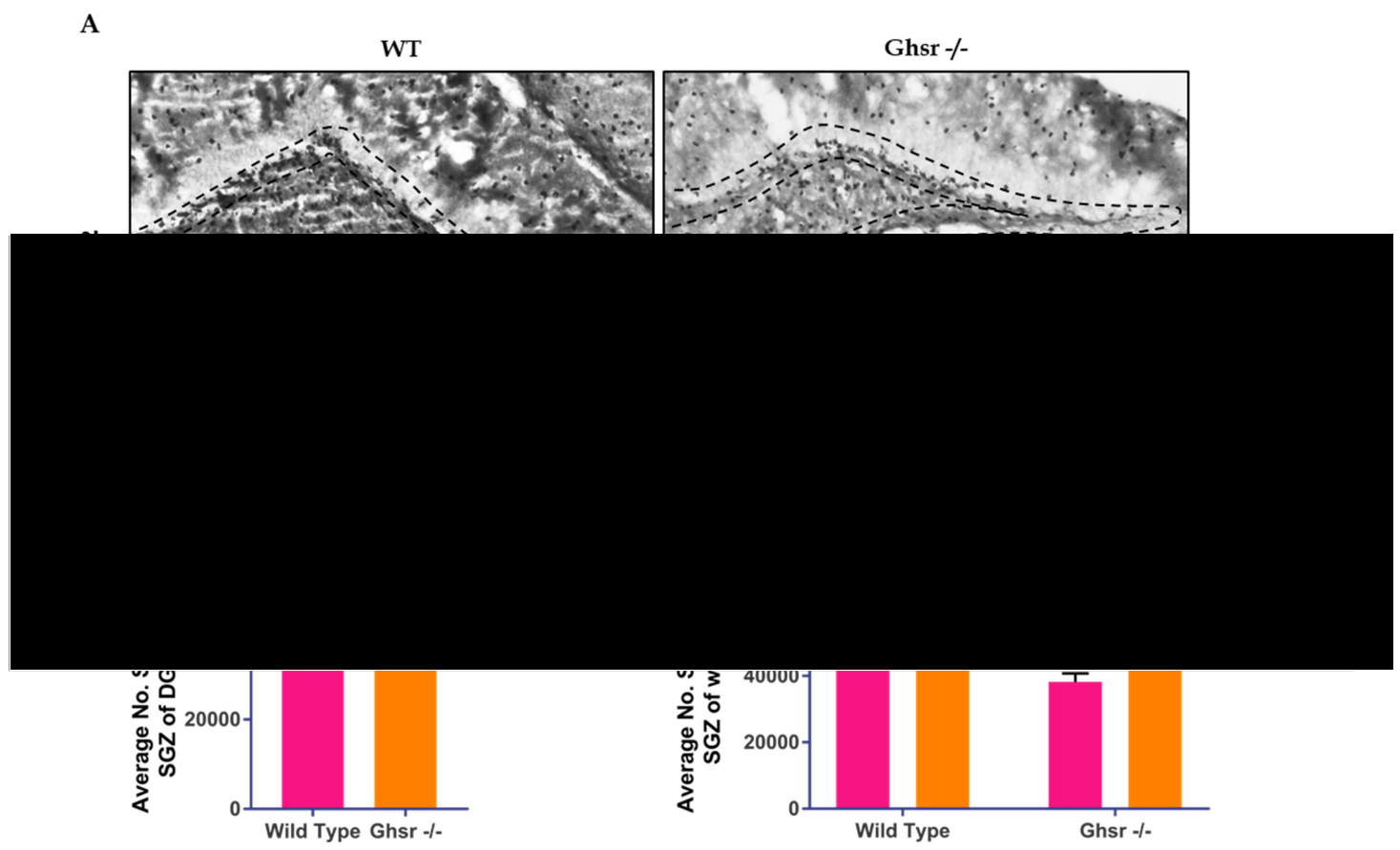

Figure 4.7 Genotype has no effect on the number of type II NSPC's, and CR significantly increases type II NSPC number independent of GHSR.

Representative immunohistochemical Ni-DAB staining of type II Sox $2^{+}$cells in the DG (A). Dashed line $=$ SGZ. Scalebar $=100 \mu \mathrm{m}$. No significant difference in Sox $2^{+}$cell number between WT and GHSR KO mice (B). Statistical analysis performed, Mann-Whitney test, $\mathrm{n}=8$ per group. CR has no effect on WT Sox $2^{+}$cell number, but elicits a significant increase in type II NSPC number in the GHSR KO mice, $p=$ 
0.0338. Statistical analysis performed, 2-way ANOVA followed by Tukey's multiple comparison posthoc test, $\mathrm{n}=4$ per group.

\subsubsection{The absence of circulating ghrelin has no effect on the numbers of type II NSPC's, proliferating cells, immature and new-born neuronal cells in the DG.}

As we demonstrate that the ghrelin receptor, GHSR, appears to be important in promoting the beneficial effects of CR on AHN and cognitive function, we sought to determine whether circulating ghrelin mediates this action.

To analyse whether the genetic ablation of ghrelin affects AHN Ghrelin KO mice, that lack both active (AG) and inactive ghrelin (UAG), and their WT littermates were analysed by IHC for markers of type II NSPCs (Sox $2^{+}$, Figure 4.8A), proliferating cells $\left(\mathrm{Ki}_{67^{+}}\right.$, Figure $\left.4.8 \mathrm{~B}\right)$ and immature neuronal cells $\left(\mathrm{DCX}^{+}\right.$, Figure $\left.4.8 \mathrm{C}\right)$. Immunofluorescent staining was also performed for new adult-born mature neurones (BrdU+ $\mathrm{NeuN}^{+}$, Figure 4.8D). Statistical analysis of cell counts (2-tailed Students $t$-test) revealed no significant difference between WT and ghrelin $\mathrm{KO}$ mice in any of the comparisons made. These data suggest that the absence of circulating ghrelin has no effect on rates of basal AHN. 


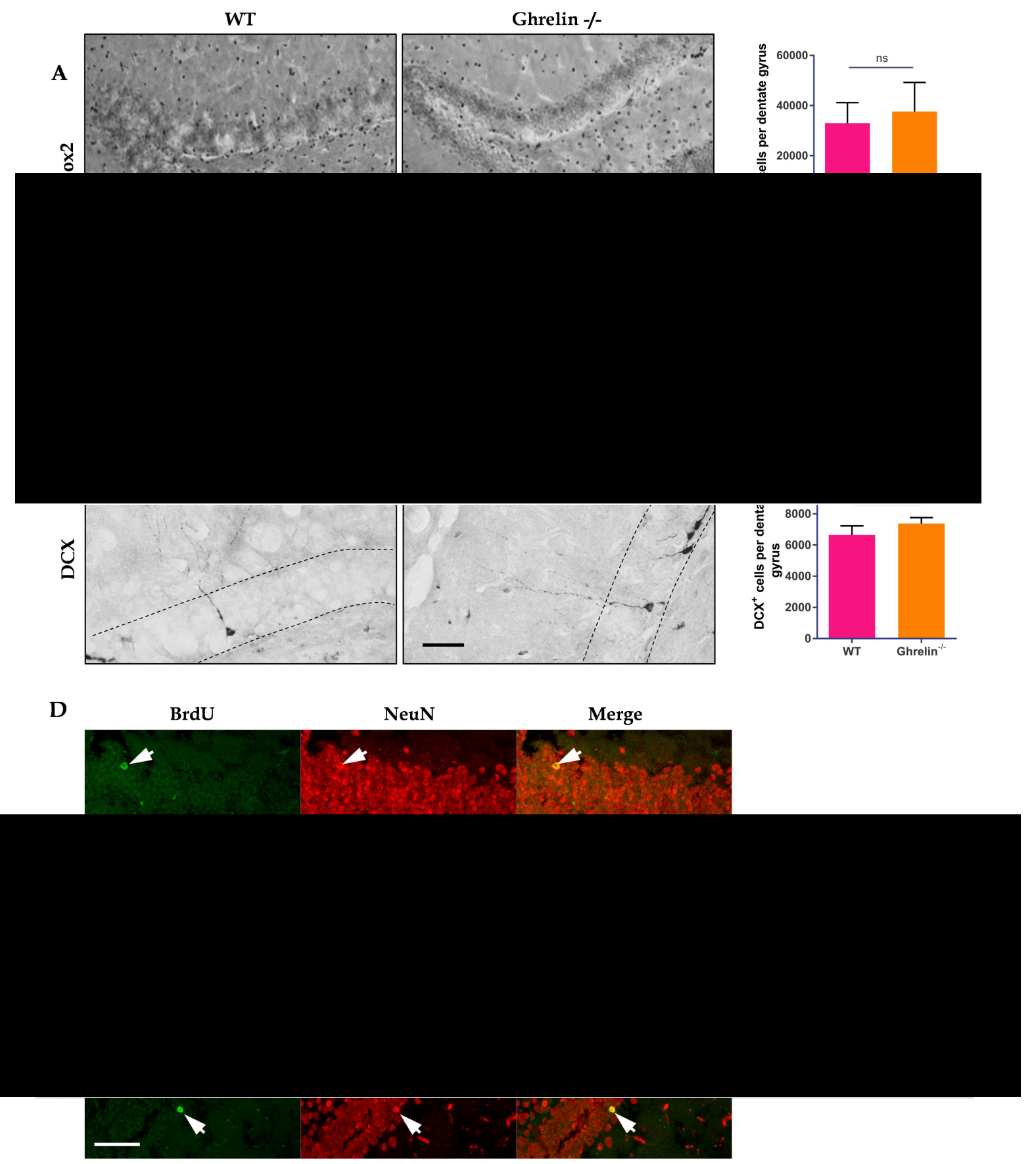

Figure 4.8 Ghrelin KO mice, lacking in circulating AG and UAG, show no effects on basal rates of neurogenesis in the dentate gyrus.

Ghrelin KO mice show no change in the number of Sox2+ NSPCs (n=8/group)(A), proliferating Ki67+ cells $\left(n=4 /\right.$ group)(B), immature $\mathrm{DCX}^{+}$neurones (WT $n=6$. ghrelin $\left.\mathrm{KO} n=7\right)(\mathrm{D})$, and new-born $\mathrm{BrdU}^{+} \mathrm{NeuN}^{+}$mature neurones (WT $\mathrm{n}=9$, ghrelin $\left.\mathrm{KO} n=12\right)(\mathrm{D})$, in the DG of the hippocampus. Scale bar $=200 \mu \mathrm{m}$ for $\mathrm{A} \& \mathrm{~B}, 50 \mu \mathrm{m}$ for $\mathrm{C} \& \mathrm{D}$, white arrow indicates $\mathrm{BrdU}+\mathrm{NeuN}^{+}$cell. Statistical analysis was done by 2 -talied Student $t$-test. Scalebar $=200 \mu \mathrm{m}(\mathrm{A} \& B), 50 \mu \mathrm{m}(\mathrm{C} \& \mathrm{D})$. ns $=$ not significant. All data represented as mean \pm SEM (Hornsby et al., 2018). 


\subsubsection{Ghrelin KO mice show normal rate of new adult-born neurone activation following $C R$.}

To determine the effect of ghrelin $\mathrm{KO}$ on new adult-born neurone activation, hippocampal sections were immunofluorescently labelled with BrdU and the IEG marker c-Fos (Figure 4.9B, C), a marker of cell activation. Immunoreactivity following $\mathrm{CR}$ and CR-refeeding was also measured, to determine whether ghrelin mediates this cell activation. Analysis of the data by 2-way ANOVA and Tukey's post-hoc multiple comparison tests showed there was no effect of genotype, $\mathrm{F}(1,33)=0.02195, \mathrm{P}=0.8831$. With regard to feeding regime, there was a significant increase in the number of $\mathrm{BrdU}^{+} \mathrm{C}^{-}$ Fos $^{+}$cells in the WT CR group compared to WT ad-lib controls ( $\mathrm{P}=0.0444$, Figure 4.9D), this was also observed between the ghrelin KO CR group and the ghrelin $\mathrm{KO}$ ad-lib group ( $\mathrm{P}=0.0235)$. This indicates that these new-born neurones' activity is sensitive to CR. Also, there was a significant increase in the number of double positive cells following CR-refeeding in the WT group compared to ad-lib fed WT controls $(\mathrm{P}=0.0288)$. From this analysis the overall effect of the feeding paradigm was highly significant, $F(2,33)=12.83$, $\mathrm{P}<0.0001$, indicating that $\mathrm{CR}$ had an overall positive effect on new cell activation, be it independent of the presence of ghrelin. With regards to the subset of new-born cells that were inactive $\left(\mathrm{BrdU}^{+}{ }^{+}\right.$-Fos', Figure 4.9E), these were not affected by genotype, $\mathrm{F}(1,33)=0.1967, \mathrm{P}=0.6603$, but their response to the feeding paradigm was significant across both WT and ghrelin KO groups, $\mathrm{F}(2,33)=5.764, \mathrm{P}=0.0071$. This indicates that the absence of circulating ghrelin does not affect the rate of new-born cell production that are not activated by CR. It was also observed that there was no effect of genotype on the total number of activated cells in the DG (c-Fos ${ }^{+}$, Figure $4.9 \mathrm{~F}), \mathrm{F}(1,33)=1.321, \mathrm{P}=0.2587$. In addition, there was no significant response to feeding pattern in these cells (Figure 4.9F), F $(2,33)=1.431$, $\mathrm{P}=0.2536$. The total number of new-born cells in the $\mathrm{DG}\left(\mathrm{BrdU}^{+}\right)$was significantly increased by CR in both WT (WT ad lib vs WT CR, P = 0.0177) 
and ghrelin $\mathrm{KO}^{\prime} \mathrm{s}$ (ghrelin $\mathrm{KO}$ ad lib $v$ s ghrelin $\mathrm{KO} C \mathrm{CR}, \mathrm{P}=0.0026$ ), showing that the effect of $\mathrm{CR}$ on new cell production is significant (feeding pattern, $\mathrm{P}$ $<0.0001, \mathrm{~F}(2,31)=15.18)$, independent of genotype $(\mathrm{P}=0.5132, \mathrm{~F}(1,31)=$ 0.4376) (Figure 4.9G). These data suggest that there may be another mediator of the beneficial effects of $\mathrm{CR}$ on the activation of these new-born cells in the DG of the hippocampus. 
$\mathbf{A}$

ArdU

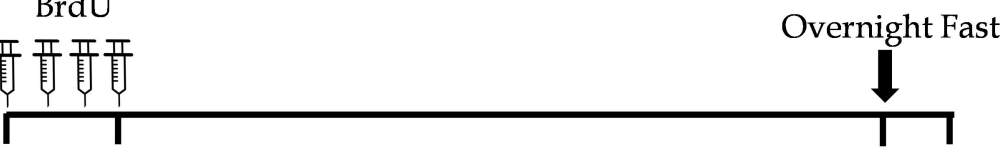

$\begin{array}{llrl}\text { Day } 14 & 27 & 28\end{array}$

$1 / 2$ Fasted mice refed $1 \mathrm{hr}$, before $\mathrm{X}$.

B

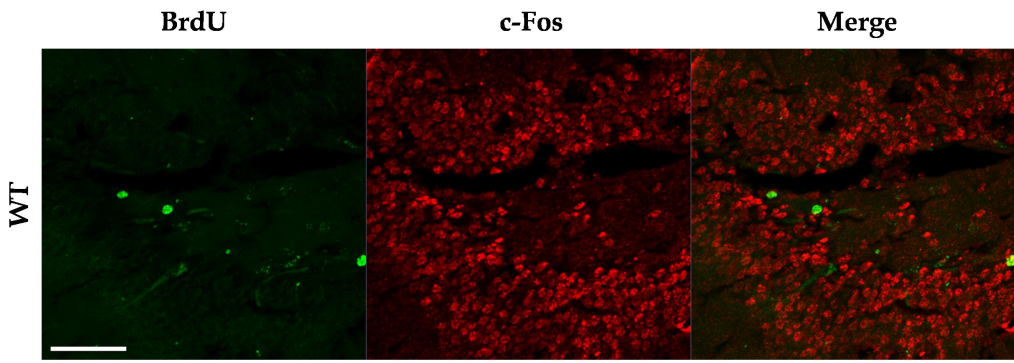

$\mathrm{C}$
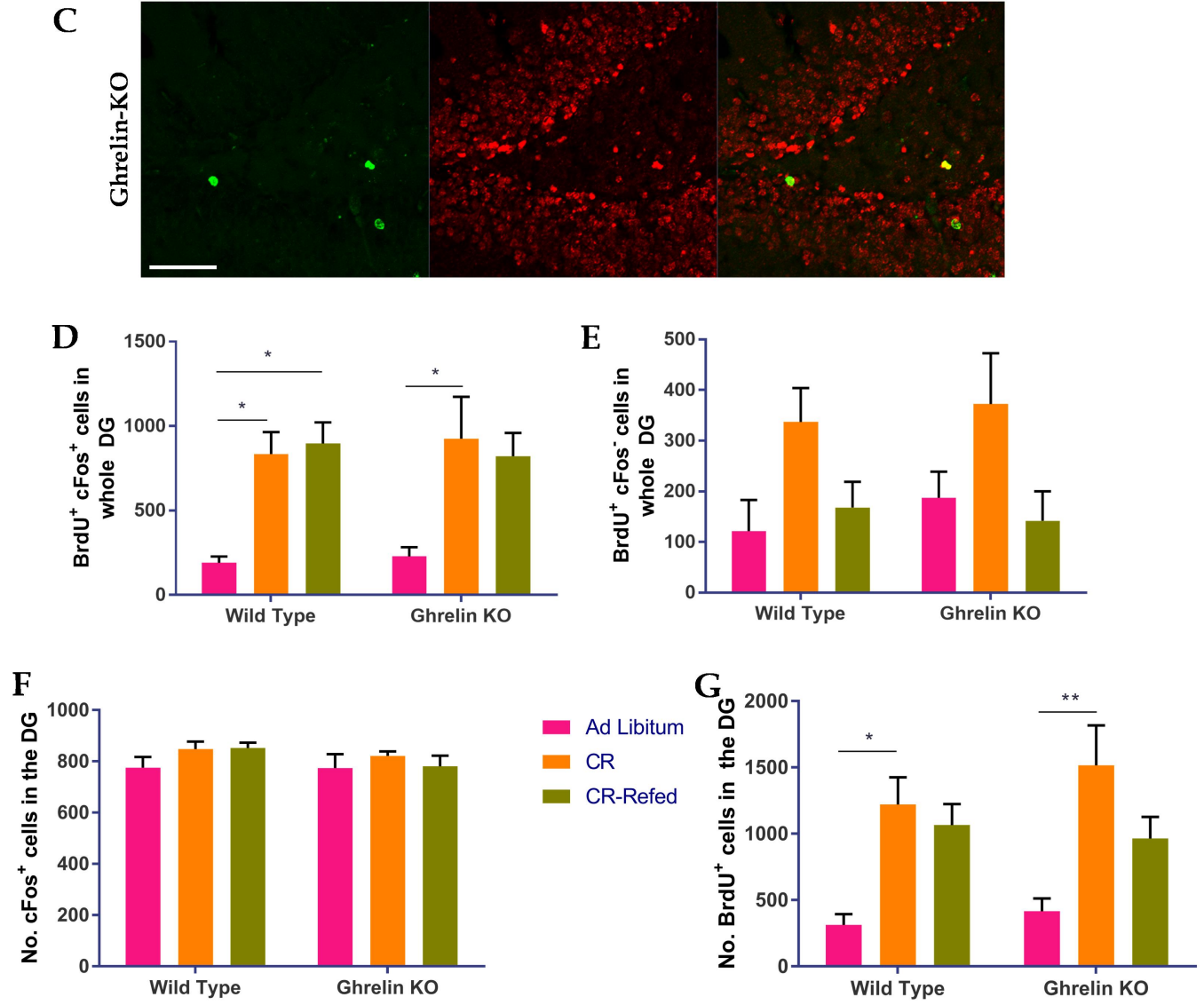

Figure 4.9 Mice lacking in circulating AG and UAG have no impairment in their response to CR.

Experimental paradigm schematic (A). Representative confocal images of new adult-born active cells $\left(\mathrm{BrdU}^{+} \mathrm{c}_{-} \mathrm{Fos}^{+}\right)$in WT (B) and Ghrelin KO (C) mice. Genotype has no effect on BrdU+ $\mathrm{c}^{-F o s}{ }^{+}(\mathrm{D})$, or the number of BrdU ${ }^{+}$c-Fos- cells (E). Total c-Fos cell number in the DG was unaffected by genotype or feeding regime (F). Total BrdU cell number in the DG was increased by CR in both WT and KO (G). Scalebar $=50 \mu \mathrm{m} . \mathrm{n}=6 /$ group. Statistical analysis performed 2-way ANOVA followed by post-hoc Tukey's multiple comparisons test. ${ }^{*}=p<0.05,{ }^{* *}=p<0.01$. All data represented as mean \pm SEM. 


\subsubsection{The effect of age on new neuronal cell survival and activation.}

Due to our interest in CR, AHN and cognitive decline in an aging population the effect of age was also analysed in these ghrelin $\mathrm{KO}$ mice and their WT littermates. Adult mice aged 6-7 months were compared to an aged group of 15-17 months of age, to determine whether age affected the rate of new adult-born neurone survival $\left(\mathrm{BrdU}^{+} \mathrm{NeuN}^{+}\right)$and new adult-born cell activation $\left(\mathrm{BrdU}^{+} \mathrm{c}-\mathrm{Fos}^{+}\right)$, in the presence and absence of ghrelin. This analysis consisted of 4 different groups; WT 6-7 month $(\mathrm{n}=5)$, WT 15-17 month $(\mathrm{n}=4)$, ghrelin KO 6-7 month $(\mathrm{n}=6)$ and ghrelin KO 15-17 month $(\mathrm{n}=$ $5)$, that underwent overnight CR. The cell counts were statistically analysed using a 2-way ANOVA followed by Tukey's post-hoc multiple comparisons test. The overall effect of genotype and age was not significant for BrdU+ $\mathrm{NeuN}^{+}$cells (Figure 4.10A, E); genotype $\mathrm{F}(1,16)=1.15, \mathrm{P}=0.2994$, and age $\mathrm{F}(1,16)=0.03643, \mathrm{P}=0.8510$. However, a subset set of those new adult-born $\mathrm{BrdU}^{+} \mathrm{NeuN}-$ cells - which are not mature neurones - do appear to be sensitive to age (Figure 4.10B, E). Indeed, there was a significant increase in the number of BrdU' $\mathrm{NeuN}^{-}$cells in the aged mice compared to young, in both WT (WT 6-7 month vs WT 15-17 month, P=0.0143) and ghrelin KO (Ghrelin KO 6-7 month vs Ghrelin KO 15-17 month, $\mathrm{P}<0.0001$ ) mice. This suggests an increase in new-born cell survival with a decrease in AHN and a potential increase in NSPC formation and or gliogenesis with advancing age. Similarly, there is no significant effect of age on new adult-born active cells $\left(\mathrm{BrdU}^{+} \mathrm{c}^{-F_{0}+}{ }^{+}\right.$, Figure 4.10C, F), F(1,18) $=0.3184, \mathrm{P}=0.5795$. Conversely, there does appear to be a significant difference within the aged groups of mice (WT 15-17 month vs Ghrelin KO 15-17 month), with an elevation in the number of $\mathrm{BrdU}^{+} \mathrm{c}-\mathrm{Fos}^{-}$cells in the $\mathrm{DG}(\mathrm{P}=0.0455)$, and difference in genotype overall $(\mathrm{F}(1,18)=5.48, \mathrm{P}=0.0309)$ (Figure 4.10D, $\mathrm{F}$ ). This suggests that there may be another soluble mediator promoting this new adult-born cell survival, in aged mice, in the absence of circulating ghrelin. 
A

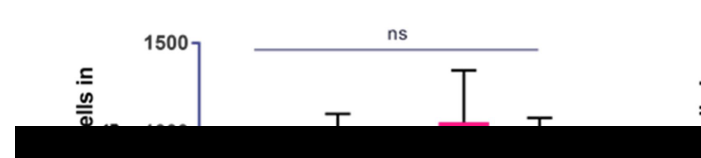

B

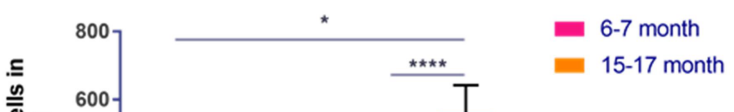

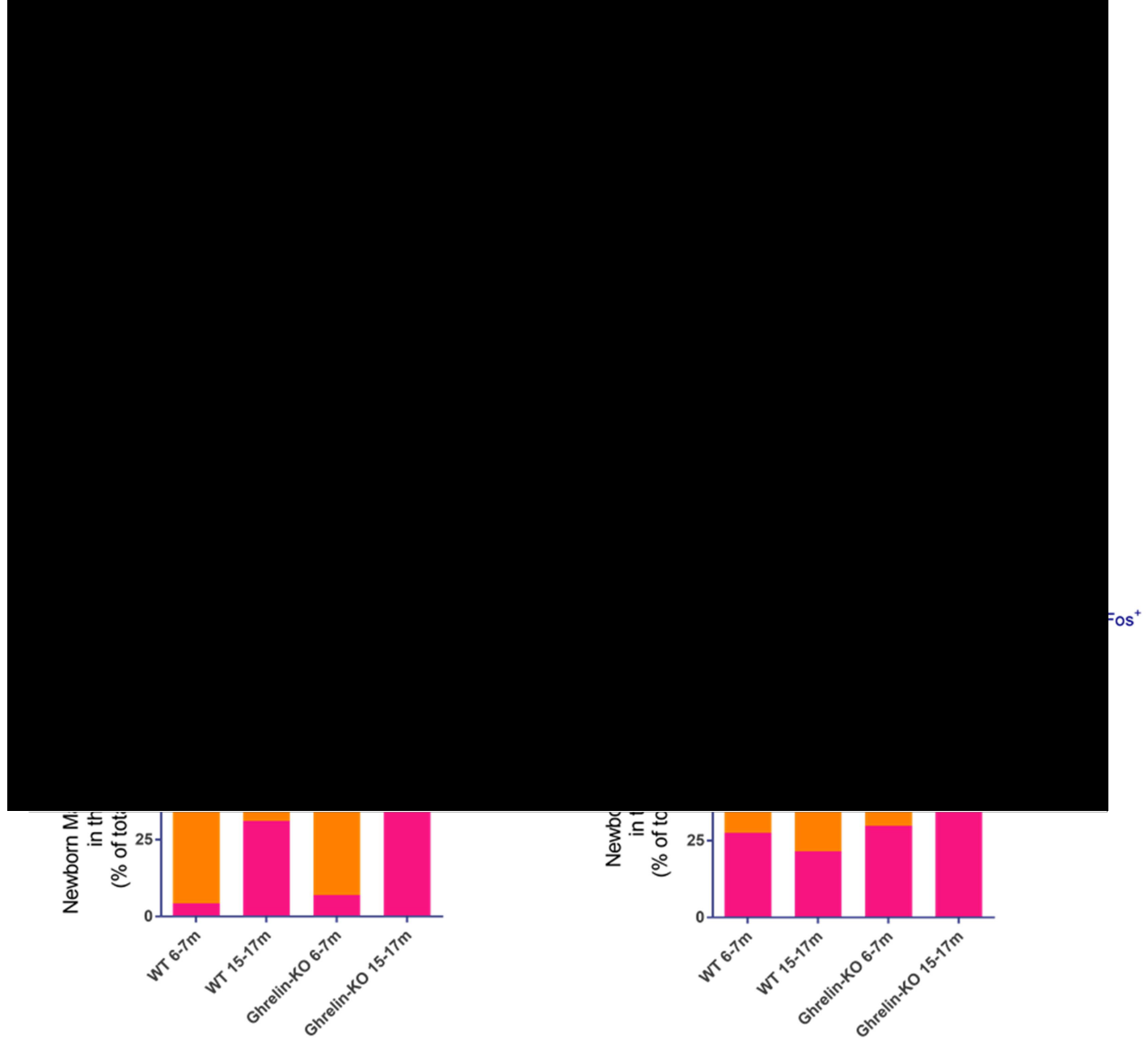

Figure 4.10 Age has no overall effect on the number of new adult-born neurones and new adultborn active cells in CR mice.

No significant difference in the survival of new-born mature neurones $\left(\mathrm{BrdU}^{+} \mathrm{NeuN}^{+}, \mathrm{A}\right)$ or new-born cell activation $\left(\mathrm{BrdU}^{+} \mathrm{c}-\mathrm{Fos}^{+}, \mathrm{C}\right)$ seen with age or genotype. However, the number of new non-neuronal cells (BrdU' $\left.{ }^{+} \mathrm{NeuN}^{-}\right)$affected increased by with age in both CR-ed WT and ghrelin KO (B). With new cells that are not active (BrdU+ c-Fos-, D) there is a significant difference between the 15-17 month CRed WT and ghrelin KO mice. Percentage proportion of new-born cells that are activated (F) and become mature neurones (E). Statistical analysis performed by 2-way ANOVA followed by Tukey's post-hoc multiple comparisons test. $\mathrm{n}=4-6$ /group. $\mathrm{ns}=$ not significant, ${ }^{*}=\mathrm{P}<0.05,{ }^{* * * *}=\mathrm{P}<0.0001$. 


\subsection{Discussion}

Different transgenic mouse models were used to decipher ghrelin's mode of action; GHSR-eGFP mice and GHSR KO mice were used to define whether the presence of GHSR was necessary to facilitate the positive effects of $\mathrm{CR}$ and/or AG treatment on AHN. Ghrelin KO mice were used to determine if ghrelin is the circulating mediator that promotes these beneficial pro-neurogenic effects on basal rates of AHN and new cell activation in the DG.

This work has shown that by elevating endogenous ghrelin levels by $\mathrm{CR}$ and exogenously via AG treatment, promotes AHN and neuronal cell activation in cells that express ghrelin receptor in the DG, and that the presence of GHSR is needed to facilitate this (Figure 3.4). Interestingly, the absence of circulating AG and UAG had no effect on basal rates of AHN or new adult-born cell activation in the DG, suggesting that ghrelin's action on neurogenesis and cell activation may not be direct. Ghrelin could possibly induce other soluble factors that promote $\mathrm{AHN}$ and aid in memory consolidation, such as BDNF (Bekinschtein et al., 2013, 2014).

Ghrelin treatment has been shown to induce the expression of IEG's Egr-1 and c-Fos in the arcuate nucleus of fasted and fed mice (Hewson \& Dickson, 2001). With this knowledge, CR's and AG treatment's effect on cell activation in cells that express GHSR in areas of the brain that are important for learning and memory was examined. This work showed that both $\mathrm{CR}$ and AG treatment elicits a significant increase in the expression of Egr-1 on cells that express GHSR and those that do not, in the DG. This suggests that GHSR activation induces Egr-1 expression in the DG. This is interesting, as Egr-1 expression specific to the DG is known to be important for the functional integration on new-born neurones in the DG. In a study using zif268 KO mice (Egr-1 KO) and WT mice (male, 2-3 months old) were administered $\mathrm{BrdU}(50 \mathrm{mg} / \mathrm{kg})$ to identify the effects of the $\mathrm{KO}$ on cell 
proliferation, survival, and survival after memory testing (morris watermaze - locate a hidden platform). It was shown that zif268 (Egr1) was required for the survival of new-born cells in the 2-3 week period after their birth, as zif268 KO mice showed decreased BrdU+ cells in the DG 21 days post injection (dpi) compared to WT, indicating increased apoptosis. However, this accelerated death didn't affect the number to immature and mature neurones. Also, it was observed following training in the morris water-maze task, that WT mice spent more time in the correct quadrant for the platform than the $\mathrm{KO}$ mice, and significantly more crossings in the right area compared to KO's, indicating that Egr1 is required for spatial learning and memory. Also, the dentate granule cells (DGC) in $\mathrm{KO}^{\prime}$ s at $21 \mathrm{dpi}$ had a reduced GluR1 expression (when they should be receiving glutamatergic inputs) compared to WT and an imbalance in the expression of chloride cotransports NKCC1 and KCC2, meaning that there is a delay in the maturation of GABA- and glutamatergic functions of these new-born DGC's (Veyrac et al., 2013). So, this increased expression of Egr1 following CR, which increases ghrelin levels, may promote the survival of these new-born neurones, allowing more new-born cells to mature and functionally integrate.

c-Fos expression was also significantly increased in the DG by AG treatment but not by CR. Egr-1 expression was also elevated in other areas of the brain important for learning and memory; the lateral entorhinal cortex, cingulate cortex and the basal lateral amygdala, following both AG treatment and the combination of AG treatment and CR. This is interesting as Egr-1 is known to have a function in synaptic plasticity and hippocampal memory formation (Bozon, Davis, \& Laroche, 2002). The work in this chapter has shown that AG increased c-Fos expression in the DG, on both GHSR- and $\mathrm{GHSR}^{+}$cells. Also, in the experiments in ghrelin $\mathrm{KO}$ mice, overnight fasting did significantly induce c-Fos expression, but only in new-born neurones. These data suggest that targets of c-Fos could also be mediating ghrelin/GHSR signalling. A recent study has looked at various stimuli that 
target c-Fos in regards to neuronal activity. Mouse coritcol neurones in culture were treated with $\mathrm{KCl}(55 \mathrm{~nm}), \operatorname{BDNF}(10 \mathrm{ng} / \mathrm{ml})$ and forskolin (adenylate cyclase stimulator, $10 \mu \mathrm{M}$ ), and all showed activation of c-Fos enhancers (e1-e2-e5, e1-e4-e5 and e1-e5, respectively) and increased c-Fos mRNA levels. Also, BDNF treatment in the mouse brain induced activation of c-Fos enhancers in the hippocampus (Joo, Schaukowitch, Farbiak, Kilaru, \& Kim, 2015).

Ghrelin has been shown to have a proliferative effect on hippocampal cells (Johansson et al., 2008) and neural progenitor cells (Li et al., 2014; Li, Chung, et al., 2013). However, from the data produced in this experiment indicated that $\mathrm{CR}$ and/or $\mathrm{AG}$ treatment had no effect on the rates of type I $\left(\mathrm{Ki}^{6} 7^{+} \mathrm{Nestin}^{+}\right)$and type II $\left(\mathrm{Ki}^{+} 7^{+}\right.$Sox $\left.2^{+}\right)$NSPC proliferation, and had no effect on overall proliferation rates $\left(\mathrm{Ki} 67^{+}\right.$cells), suggesting that acute AG treatment and a short period of CR (these mice were fasted for 16 hours, overnight) has no effect on cell proliferation. In comparsion to previous studies, this work shows a similar outcome to that of Yumi Kim who demonstrated that 3 months of $\mathrm{CR}$ had no effect on progenitor cell proliferation in WT and ghrelin KO mice (Kim et al., 2015), but conflicts with the work in Mark Mattson's lab showing that CR increased proliferation of cells (J Lee et al., 2000).

In light of the effect of overnight CR on IEG expression, a 2-week period of CR was used to understand the effects of longer-term CR on AHN. We show that this prolonged period of CR increases the number of new adult-born mature neurones $\left(\mathrm{BrdU}^{+} \mathrm{NeuN}^{+}\right)$in the DG of the hippocampus, 31 days after the last day of CR. This effect was not seen in GHSR KO mice, but in their WT littermates, showing that the receptor is required to facilitate the pro-neurogenic effects of CR. As ghrelin is not produced in the brain (Sakata et al., 2009), it may be circulating ghrelin that is crossing the blood brain barrier to modulate AHN, as has been shown by William Banks who that both AG and UAG can cross the BBB (Banks et al., 2002) and Tamas 
Horvath showed that peripheral ghrelin acts on the hippocampus (Diano et al., 2006), or other unidentified ligands that interact with GHSR within the DG such as GHRH (growth hormone releasing hormone) a known agonist of GHSR (Casanueva et al., 2008). However, AHN is enhanced by peripheral treatment with AG (Kent et al., 2015) and CR increases ghrelin levels in plasma (Lutter et al., 2008), so it is highly likely that the pro-neurogenic effects of CR and the requirement of GHSR to facilitate this, is stimulated by ghrelin.

These GHSR KO mice also showed deficits in retaining fear memory, which has been shown previously by Albarran-Zeckler et al. 2012,. CR showed to alleviate these deficits with GHSR KO mice, by demonstrating improvements in fear-memory retention. Contextual fear conditioning (CFC) is a hippocampal dependant test for learning and memory and sensitive to AHN (Kitamura et al., 2009), and the new mature neurones produced by AHN are important for pattern separation memory (Clelland et al., 2009).

Although ghrelin has previously been shown to stimulate $\mathrm{AHN}$ in ghrelin $\mathrm{KO}$ mice and promote the migration and division of NSPC's (Li et al., 2014; Li, Chung, et al., 2013), this was not observed the ghrelin KO model that was used in this analysis. In addition, the absence of circulating AG and UAG had no effect on type II NSPC number, the rate of cell proliferation or the production of new adult-born neurones in the DG. Furthermore, CR had no effect on new adult-born cell activation in this mouse model. This may be due to experimental differences. Our study used female C57BL/6 ghrelin KO mice that were ages between 5 and 16 months old, whereas Li et al used male C57BL/ 6 mice aged 8-9 weeks. So, there are differences in sex and age of mice used for each study. Also, ghrelin was elevated in our study via an overnight fast before being terminally anaesthetized, and the brain tissue was immersion fixed in PFA. Whereas the $\mathrm{Li}$ et al mice received ghrelin injections $(80 \mu \mathrm{g} / \mathrm{kg})$ for 8 days and were transcardially perfused with PFA. So, the route by which ghrelin was elevated were different and the way in 
which the tissue was fixed was different. All factors that may affect the outcome of an experiment.

A limitation of the ghrelin $\mathrm{KO}$ model used in this chapter is it does not have any circulating AG and UAG, but it also lacks obestatin. Treatment with obestatin in male Wistar rats produced improvements in novel object recognition behavioural memory testing with increased exploration, which is dependent on the hippocampus, and also an increase in latency time in the step-down test indicating increased memory retention (Carlini, Schiöth, \& deBarioglio, 2007). Obestatin has also shown to enhance the proliferation of adult hippocampal progenitor cells (AHPs) in vitro. Rat AHPs treated with various concentrations of obestatin $(1,10,100$ and $500 \mathrm{nM})$ all showed a significant increase in cell proliferation and survival, and also a reduction in caspase 3 activity indicating anti-apoptotic effects also (Gargantini et al., 2016).

The GHSR KO mouse model also has its limitations. This is a germline $\mathrm{KO}$; meaning that this mouse model underwent development without GHSR, so potentially there may have been some developed compensatory mechanisms to counteract this. Newer technologies that are chemogenetically based, such as DREADDs (designer receptors exclusively activated by designer drugs) could be of better use, as you would have control over when to $\mathrm{KO}$ a particular gene. These are being more readily used in neuroscience and signalling (Urban \& Roth, 2015).

These data suggest that $\mathrm{CR}$ mediates positive effects on $\mathrm{AHN}$ and learning and memory through GHSR signalling. However, ghrelin may not be the only peptide that triggers its downstream effects. 


\section{Chapter 5 The effect of UAG and AG on}

\section{AHN}

\subsection{Introduction}

The most abundant form of ghrelin in the circulation is the so-called inactive form, UAG, which makes up approximately $90 \%$ of circulating ghrelin. UAG is activated via the addition of an octanoyl group, and this octanoylation is executed by the enzyme GOAT (ghrelin-o-acyl transferase) in the stomach (Gutierrez et al., 2008; Yang et al., 2008). Following this posttranslational octanoylation AG can bind its receptor, GHSR (M Kojima et al., 1999).

More recent work has shown that ghrelin may undergo tissue specific acylation events, such as in the dentate gyrus of the hippocampus (Murtuza \& Isokawa, 2018) and bone marrow where it promotes adipogenesis (Hopkins et al., 2017). Also, AG and UAG have been shown to have a synergistic relationship, where AG promotes and UAG down-regulates glucose output of hepatocytes (Gauna et al., 2005). Furthermore, with regard to synaptic plasticity and long term potentiation (LTP), AG has shown to prolong LTP in the DG whereas UAG has no effect on LTP (L. Chen et al., 2011). These data suggest that regulating the octanoylation status of ghrelin may have important modulatory effects on ghrelin function in a tissue specific manner.

Dendritic spines are protrusions from the neuronal membrane that consist of a head connected to the dendrite by a thin neck. These spines receive connections from excitatory synapses and contain $\mathrm{Ca} 2^{+}$within these compartments which is essential for signalling and synaptic plasticity 
(Nimchinsky, Sabatini, \& Svoboda, 2002; Sabatini, Maravall, \& Svoboda, 2001).

\subsubsection{Aim of this chapter.}

As we have shown in previous chapters that elevation of AG, indirectly via $C R$ and directly via $A G$ treatment positively modulates neurogenesis in adult mouse brain, and that CR's effects are dependent on GHSR, this chapter aims to determine whether UAG modulates AHN in the hippocampus. 


\subsection{Materials \& Methods}

\subsubsection{Mice}

All animal work in this chapter on the Regeneron GOAT KO mouse model (5.2.1.2) was performed in the lab of Zane Andrews of Monash University, Melbourne, Australia. All work was carried out with ethical approval from the institution.

All animal work on the Taconic Farms GOAT KO mouse model was performed in the lab of Tim Wells of Cardiff University, UK. All work was carried out with the ethical approval from the institution.

\subsubsection{UAG treatment in WT and GOAT KO mice}

6 month old GOAT homozygous KO mice and their WT C57BL/6 littermates, generated in Taconic Farms (Hudson, NY), underwent a 7 day treatment of either vehicle (sterile isotonic saline with BSA $(1 \mathrm{mg} / \mathrm{ml})$ and heparin $(5 \mathrm{U} / \mathrm{ml})$ at $0.5 \mu \mathrm{l} / \mathrm{h}))$ or UAG $(48 \mu \mathrm{g} /$ day $)$, which was administered via an indwelling jugular vein catheter attached to a subcutaneous osmotic mini-pump (Alzet, model 1007D). 1 day after the pumps were fitted, the mice received an i.p. injection of BrdU (50mg/ $\mathrm{kg})$ to label any dividing cells. After the 7 days, mice were anaesthetised with isoflurane and sacrificed by decapitation, and the brain tissue removed.

\subsubsection{AG treatment in WT and GOAT KO mice}

12 week old adult male C57BL/6 WT and GOAT KO from Regeneron were created using Velocigene technology where the GOAT gene ATG-stop 
sequence was replaced with lac reporter gene with the BAC (bacterial artificial chromosome) (Yang et al., 2008). These mice underwent 7 days of i.c.v. (intracerebroventricular) infusions of either artificial cerebro-spinal

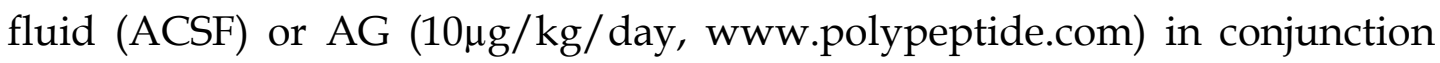
with $\mathrm{BrdU}(50 \mathrm{mg} / \mathrm{kg})$ using Alzet osmotic mini-pumps. The mice were separated into 4 groups; WT ACSF + BrdU, WT AG + BrdU, KO ACSF + BrdU and $\mathrm{KO} A G+B r d U$. At the end of day 7 the mice were intra-cardially perfused with $4 \%$ PFA and the brain tissue collected.

To allow for the longer-term analysis of treatment on AHN an additional cohort of these mice (same 4 groups) were maintained under normal laboratory conditions until day 28 , before they were intra-cardially perfused with $4 \%$ PFA and the brain tissue collected.

Finally, another subset of these WT and GOAT KO mice, that were approximately 12 weeks old, were sacrificed to undergo Golgi-Cox treatment for analysis of dentritic spine density (see 5.2.9).

\subsubsection{UAG treatment in WT mice}

12 week old WT C54BL/6 mice were given daily subcutaneous infusions of UAG $(80 \mu \mathrm{g} / \mathrm{kg} /$ day, www.polypeptide.com) for 7 days, via osmotic mini-pump (Alzet). Following the 7 days, mice were intra-caridially perfused with $4 \%$ PFA and the brain tissue collected.

\subsubsection{Tissue Preparation}

Mouse brain samples were collected following intra-cardial fixation with $4 \%$ paraformaldehyde (PFA) (Sigma Aldrich, St Louis, USA) and sunk in $30 \%$ sucrose prior to being stored at $4^{\circ} \mathrm{C}$ in the cold room, submerged in PBS (Sigma Aldrich, St Louis, USA) with 0.1\% Sodium Azide (Sigma Aldrich, 
St Louis, USA), until required for analysis. All procedures were performed with ethical approval at Monash University, Australia. Swansea University's Animal Welfare and Ethical Review Panel (AWERP) approved the studies.

\subsubsection{Tissue Sectioning}

Mouse brain tissue was sectioned as previously stated in the general methods 2.2. Sections were specifically collected into 12-well Nunclon plates, in a ratio of 1:12.

\subsubsection{Immunofluorescent staining of recently divided immature neuronal cells (BrdU-DCX) in 6 month old WT and GOAT KO mice.}

See protocol section 2.3.3, with the following specific details.

Following DNA denaturation with $2 \mathrm{M} \mathrm{HCl}$ and Borate buffer (pH6) and a series of washes, sections were blocked with 5\% NDS in $0.1 \%$ PBS-T for 60 minutes. Excess block was removed and a cocktail of both primary antibodies (Table 5.1) diluted in 0.1\% PBS-T were applied and incubated for 24 hours at $4^{\circ} \mathrm{C}$, on a rocker in the cold room. Sections were washed as before in 1X PBS, following which a cocktail of both secondary antibodies (Table 5.2) diluted in 0.1\% PBS-T was applied to the tissues, and incubated for 30 minutes. Sections were washed a final time as before, with the addition of Hoechst nuclear counterstain (1:10,000; H3570, Life Technologies) to the first wash. Finally, sections were mounted as previously described. 
Table 5.1 Primary antibodies used.

\begin{tabular}{|c|c|c|c|c|}
\hline Target & Cell Type & Species & $\begin{array}{l}\text { Company \& Cat. } \\
\text { No. }\end{array}$ & Dilution \\
\hline BrdU & New-born cells & Rat & $\begin{array}{ll}\text { ABD } & \text { Serotec } \\
(\text { MCA2060) } & \end{array}$ & $1: 400 / 1: 3000$ \\
\hline DCX & $\begin{array}{l}\text { Immature } \\
\text { neuronal cells }\end{array}$ & Goat & Santa Cruz (Sc-8066) & $\begin{array}{l}\text { 1:200 IF } \\
\text { 1:500 NiDAB }\end{array}$ \\
\hline NeuN & $\begin{array}{l}\text { Mature Granule } \\
\text { cells }\end{array}$ & Mouse & Millipore (MAB377) & $1: 1000$ \\
\hline Ki67 & $\begin{array}{l}\text { Proliferating } \\
\text { cells }\end{array}$ & Rabbit & Abcam (Ab16667) & 1:500 Ni-DAB \\
\hline Sox2 & Type II NSPCs & Rabbit & Abcam (Ab97959) & 1:1000 Ni-DAB \\
\hline c-Fos & IEG & Rabbit & Santa Cruz (Sc-52) & 1:4000 Ni-DAB \\
\hline Egr-1 & IEG & Rabbit & Santa Cruz (Sc-189) & 1:4000 Ni-DAB \\
\hline
\end{tabular}

\subsubsection{Immunofluorescent staining of newborn mature neuronal cells (BrdU-NeuN) in 12 week old WT and GOAT KO mice.}

See protocol 2.3.3, with the following additional details.

Following DNA denaturation and washes, described previously, sections were blocked in 5\% NGS (diluted in 0.1\% PBS-T) for 60 minutes. The excess block was removed and the BrdU primary antibody (Table 5.1) diluted in $0.1 \%$ PBS-T was applied for 24 hours at $4^{\circ} \mathrm{C}$ in the cold room, on a rocker. Following this incubation, the sections were washed as before, and biotinylated secondary antibody (Table 5.2) diluted 0.1\% PBS-T was applied to the sections, and incubated for 60 minutes, protected from light. Sections were washed, after which the Streptavidin conjugate diluted in $0.1 \%$ PBS-T was applied to the tissue and incubated for 30 minutes, following which the sections were washed again. Subsequently, the NeuN primary antibody 
diluted in $0.1 \%$ PBS-T was applied to the sections and incubated for 24 hours at RT. Following another wash series the corresponding fluorescent secondary antibody was applied to the sections and incubated for 30 minutes. Finally, the sections were washed as before, with the addition of Hoechst to the first wash, and sections were mounted and coverslipped as stated above.

Table 5.2 Secondary antibodies and conjugates used.

Secondary $\mathbf{A b}$

\begin{tabular}{|c|c|c|}
\hline Alexa Fluor 568 Donkey a-Goat IgG (H+L) & $\begin{array}{l}\text { Molecular Probes } \\
\text { (A11057) }\end{array}$ & $1: 500$ \\
\hline Alexa Fluor 488 Donkey a-Rat IgG $(\mathrm{H}+\mathrm{L})$ & $\begin{array}{l}\text { Molecular Probes } \\
\text { (A21208) }\end{array}$ & $1: 500$ \\
\hline Biotinylated Goat a-Rat IgG & $\begin{array}{l}\text { Vector Laboratories } \\
\text { (BA-9400) }\end{array}$ & $1: 400$ \\
\hline Streptavidin Alexa Fluor 594 conjugate & Invitrogen (S11227) & $1: 500$ \\
\hline Alexa Fluor 488 Goat a-Mouse IgG $(\mathrm{H}+\mathrm{L})$ & $\begin{array}{l}\text { Molecular Probes } \\
\text { (A11001) }\end{array}$ & $1: 500$ \\
\hline $\begin{array}{l}\text { Biotin-SP-conjugated AffiniPure Donkey a- } \\
\text { Goat IgG }(\mathrm{H}+\mathrm{L})\end{array}$ & $\begin{array}{l}\text { Jackson Laboratories } \\
(705-065-003)\end{array}$ & $1: 400$ \\
\hline Biotinylated Goat a-Rabbit IgG & $\begin{array}{l}\text { Vector Laboratories } \\
\text { (BA-1000) }\end{array}$ & $1: 400$ \\
\hline
\end{tabular}

5.2.6 Immunohistochemical staining of immature neurones (DCX) in WT and GOAT KO mice, using Ni-DAB.

See protocol 4.2.8.

Staining in this experiment was performed as previously mentioned. 
5.2.7 Immunohisotchemical staining of proliferating (Ki67) cells, type II NSPC's (Sox2) and active (Egr-1) neurones, in WT and GOAT KO mice, using Ni-DAB.

See protocol 2.3.5 for method, with specifics below.

Following the removal of excess citrate buffer, 5\% NGS block in $0.03 \%$ PBS-T was applied to the sections and incubated for 60 minutes at RT. Primary antibody for Sox2, Ki67 or Egr-1 was then applied (see Table 5.1) to the sections, diluted in $0.03 \%$ PBS-T and $2 \%$ NGS, this was left to incubate overnight at $4^{\circ} \mathrm{C}$ in the cold room, on a rocker. Sections were washed as before and secondary biotinylated antibody (see Table 5.2) diluted in $0.03 \%$ PBS-T and 2\% NGS, was applied to the sections and incubated as stated previously.

\subsubsection{Immunohistochemical staining of active (c-Fos) neurones in WT and GOAT KO mice, using Ni-DAB.}

Please refer to the standard protocol in the methods section 2.3.4, with the following specifics.

Sections were blocked in 5\% NGS in $0.03 \%$ PBS-T for 60 minutes at RT. Following this, the primary anti-Egr-1 antibody diluted in $0.03 \%$ PBS-T with $2 \%$ NGS was applied to the sections and incubated overnight at $4^{\circ} \mathrm{C}$ on a rocker. Sections were washed in 1X PBS and 0.03\% PBS-T as before, after which sections were incubated in the biotinylated secondary antibody diluted in $0.03 \%$ PBS-T with $2 \%$ NGS for 70 minutes at RT, protected from light. Following this, the sections were washed again and a pre-mixed ABC solution was applied and sections incubated for 90 minutes at RT, protected from light. To enhance the immunoreactivity, an additional amplification step was used; sections were incubated in biotinylated tyramine (bTyr) (diluted 1:100 in $0.03 \%$ PBS-T with $0.1 \%$ Triton-X) for 10 minutes at RT. 
Sections were then washed 1 time in 1X PBS and 2 times in 0.03\% PBS-T, 10 minutes each, after which another $\mathrm{ABC}$ solution was applied to the sections for 90 minutes at RT. Subsequently, the sections were washed once in $1 \mathrm{X}$ PBS, twice in $0.03 \%$ PBS-T and once in sodium acetate for 10 minutes each. Sections were then reacted in Ni-DAB and processed as previously stated.

\subsubsection{Impregnation of WT and GOAT KO mouse brains with Golgi Cox solution.}

Golgi Cox staining of mouse brain tissue was performed using the FD Rapid GolgiStain TM Kit (PK401A, FD Neurotechnologies Inc.). Upon removal from the skull, brain tissue was rinsed quickly with Milli-Q water to remove any residual blood. The tissue was immersed in impregnation solution (equal volumes solution $\mathrm{A}$ and solution $\mathrm{B}$, which contain mercuric chloride, potassium dichromate and potassium chromate, and stored at RT for 2 weeks, in the dark. After the first 6 hours or after 1 day, the impregnation solution was changed. The tissue was then transferred into solution $\mathrm{C}$ and stored at RT for 72 hours or up to 1 week, in the dark. The solution C was replaced after the first 24 hours of immersion.

The tissue was then rapidly frozen before sectioning by slowly dipping the tissue into pre-cooled iso-pentane, on dry-ice, for a few seconds. The tissue was placed on dry-ice for 1 minute, before being individually wrapped in foil and placed in a $-80^{\circ} \mathrm{C}$ freezer for storage.

\subsubsection{Sectioning and processing of Golgi-Cox impregnated brain tissue.}

WT and GOAT KO mice impregnated with Golgi-Cox were sectioned using a Leica CM1900 Cryostat with the chamber temperature set to $-15^{\circ} \mathrm{C}$ and the cutting stage temperature set to $-10^{\circ} \mathrm{C}$. Tissue was cut to a thickness 
of $120 \mu \mathrm{m}$ and placed onto glass slides that were rinsed in Solution $\mathrm{C}$ from the FD Rapid GolgiStain ${ }^{\mathrm{TM}}$ Kit (PK401A, FD Neurotechnologies Inc.). Slides were dried overnight at RT, and protected from light. The slides were processed in the following way; sections were rinsed 2x with Milli-Q water, 4 minutes each, they were then placed in a mixture of 1 part Solution D, 1 part Solution E and 2 parts Milli-Q water (DEQ solution) for 10 minutes. The slides were then dehydrated in a series of 50\%, 75\% and 95\% EtOH for 4 minutes each, followed for $4 \times 4$ minutes in 100\% EtOH. Subsequently, the slides were delipified in Histoclear 3x 4 minutes, and coverslipped using Entellan mounting media.

\subsubsection{Analysis of fluorescent immunoreactivity.}

Fluorescently labelled tissue sections were analysed for their immunoreactivity using a Zeiss Imager M1 microscope with Axiocam MRm. High resolution imaging of co-localised proteins throughout the z-axis was performed using a Laser Scanning Confocal Microscope (LSCM) (Zeiss LSM 710).

\subsubsection{Analysis of Ni-DAB immunoreactivity.}

Ni-DAB immuno-labelled sections were analysed for their immunoreactivity using a Nikon Eclipse 50i microscope system and NIS Elements F4 software. Cells were either counted manually where possible, or with the use of ImageJ software. 


\subsubsection{Analysis of Golgi-Cox staining.}

Dendritic spines were analysed from these slides using the Nikon Eclipse 50i microscope using a Nikon Plan 100x/1.25 oil objective objective. The images were then subsequently processed using ImageJ software in order to manually count dendritic spines. Spines were categorised into the following groups; mushroom, thin spine, stubby spine, branched and filopodium (Harris, Jensen, \& Tsao, 1992; Hering \& Sheng, 2001).

\subsubsection{Data and Statistical Analysis.}

\subsubsection{Effect of UAG treatment on AHN in WT and GOAT KO mice.}

For the analysis of immunohistochemical Ni-DAB staining of proliferating cells (Ki67), immature neurones (DCX) and neuronally active cells (c-Fos), $\mathrm{n}=5-6$ per group and a whole well of hippocampal sections (approximately 10-16 sections per mouse) stained. Cells in the GCL of the DG were counted using ImageJ software (5.2.12); total cell counts for each mouse were divided by the number of sections counted to obtain an average.

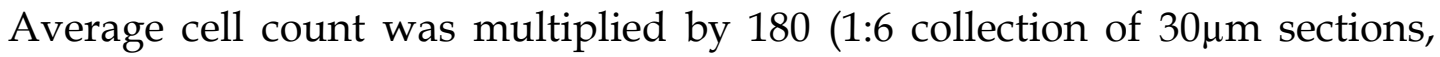
$180 \mu \mathrm{m}$ between each section) to obtain number of positive cells per whole DG.

For the analysis of immunofluorescent staining of new-born immature neurones (BrdU-DCX), $n=5-6$ per group and 4 DG sections selected for each mouse, and data handled as described in 5.2.11 and above.

Statistical analysis of the data was performed with Graphpad Prism. Due to there being 2 variables (genotype and treatment) a 2-way ANOVA followed by Holm-Sidak post-hoc multiple comparison test was used. 
5.2.14.2 Effect of UAG treatment on Type II NSPC pool in WT and GOAT KO mice.

Analysis of immunofluorescent staining of type II NSPCs (Sox2), $\mathrm{n}=4$ per group and 4-6 hippocampal sections stained. Data was collected and handled as described above (5.2.14.1). As there was one variable (genotype) a 2-tailed unpaired $t$-test was performed on the data.

\subsubsection{Effect on AHN and Type II NSPCs in second GOAT KO (Regeneron)} model.

Analysis of immunohistochemical Ni-DAB staining of proliferating (Ki67) and immature neurone (DCX) cells in the GCL of the DG, $\mathrm{n}=6$ per group and 4 sections per mouse stained. Cell counts and area measurements of the GCL were done using ImageJ software. An average cell count for each mouse was multiplied by 360; sections were collected in 1:12 ratio $(30 \mu \mathrm{m}$ sections, $360 \mu \mathrm{m}$ between sections), and divided by average area measured in $\mu \mathrm{m}^{2}$. Average number cells per $\mu \mathrm{m}^{2}$ were multiplied by 1000 to obtain average number cells per $\mathrm{mm}^{2}$.

Analysis of immunohistochemical Ni-DAB staining of type II NSPCs (Sox2), $\mathrm{n}=6$ per group and 4 sections per mouse were stained. Images were analysed (5.2.12) and data handled as described above with the exception of area measurement.

As there was one variable (genotype) a 2-tailed unpaired $t$-test was performed on the data.

\subsubsection{Effect on IEG expression in second GOAT KO model.}

For the analysis of IEG (Egr-1 and c-Fos) expression by immunohistochemical Ni-DAB staining, $n=4-8$ mice per group and 4 sections stained per mouse. Cell counts were obtained using ImageJ to analyse images taken (5.2.12). Data was handled as described above in 5.2.14.3, with 
the exception of area. Due to there being 2 variables (genotype and treatment) a 2-way ANOVA followed by Tukey's post-hoc multiple comparison tests were performed.

\subsubsection{Effect of genotype on dendritic spine formation.}

For the analysis of dendritic spines in WT and GOAT KO mice that underwent Golgi-cox treatment, $n=3$ per group and 4-6 dendrites counted per mouse. Spines were counted as stated above (5.2.13) and the dendrite length measured using ImageJ. An average of each spine type was calculated (total of each spine type / number of dendrites) and an average length of dendrite analysed, average spine number was then divided by average dendrite length to obtain average number of spine type per $\mu \mathrm{m}^{2}$ which was then $x 1000$ to obtain average spine number per $\mathrm{mm}^{2}$. Due to the multiple variables (spine type and genotype) the data was analysed statistically by a 2-way ANOVA, followed by Fishers LSD post-hoc multiple comparisons test.

\subsubsection{Effect of short-term AG treatment on AHN in WT and GOAT KO mice.}

For the analysis of immunofluorescent staining for new-born neurones (BrdU-NeuN), $\mathrm{n}=4-8$ per group with 6-8 hippocampal sections stained per mouse. Positively stained cells were counted manually as mentioned previously (5.2.11) in the GCL of the DG. Data was handled as above (5.2.14.4). Due to the 2 variables in this experiment (genotype and treatment) a 2-way ANOVA followed by Tukey's multiple comparisons test were performed on the data.

\subsubsection{Effect of UAG treatment on AHN in WT C57BL/6 mice.}

For the analysis of new-born neurones (BrdU-NeuN) in the GCL of the DG, $\mathrm{n}=9$ per group with 6-8 hippocampal sections undergoing staining. Positively stained cells in the GCL of the DG were manually counted as previously mentioned (5.2.11), and data was handled as described above 
(5.2.14.4). A 2-tailed unpaired $t$-test was performed on the data due to only one variable of treatment. 


\subsection{Results}

Part of this work was undertaken by Joesph Bhart (Ki67 and DCX staining of UAG treated WT and GOAT KO mice) and Luke Roberts (Egr-1 staining of ghrelin treated WT and GOAT KO mice), members of the Davies group. I assisted in their supervision and training of these students, and helped optimise and clarify the protocols used for both immunohistochemical staining and data analysis.

\subsubsection{UAG treatment of adult WT and GOAT KO mice leads to a reduction of $\mathrm{AHN}$.}

6 month old GOAT KO mice (see 4.2.1.1) that underwent peripheral UAG treatment had a significant reduction in the number of proliferating $\mathrm{Ki}^{+}$cells (Figure 5.1A), immature $\mathrm{DCX}^{+}$neurones (Figure 5.1C), and active ${\mathrm{c}-F o{ }^{+}}^{+}$neurones (Figure 5.2A) in the SGZ of the DG, the region where AHN occurs.

Statistical analysis of the DCX data (two-way ANOVA followed by the Holm-Sidak multiple comparisons test) revealed a main effect of genotype $(\mathrm{WT}$ and GOAT KO, P=0.0096, $\mathrm{F}(1,19)=8.287)$ and an interaction between treatment (Vehicle and UAG) and genotype (WT and GOAT -KO), $\mathrm{P}=0.0009$ and $F(1,19)=15.33$. The multiple comparisons test revealed that all other groups compared to WT-Vehicle treated mice there was a significant reduction in the number of DCX+ immature neurones in the SGZ of the DG (Figure 5.1C, D); WT-Vehicle vs WT-UAG P=0.0106, WT-Vehicle vs GOAT KO-Vehicle $\mathrm{P}=0.0003$, WT-Vehicle vs GOAT KO-UAG P=0.0195. Also, analysis of the Ki67 data revealed a significant difference between the genotypes $(\mathrm{P}=0.0175, \mathrm{~F}(1,19)=6.766)$ and the interaction between treatment and genotype $(\mathrm{P}=0.0074, \mathrm{~F}(1,19)=9.003)$ which is shown in (Figure 5.1A, B). The multiple comparisons test showed that all other groups in comparison to 
WT-Vehicle treated mice, there was a significant decrease in the number of $\mathrm{Ki}^{+}{ }^{+}$cells in the SGZ of the DG; WT-Vehicle vs WT-UAG P=0.0056, WTVehicle vs GOAT KO-Vehicle P=0.0020, WT-Vehicle vs GOAT KO-UAG P= 0.0056 .

Finally, analysis of c-Fos ${ }^{+}$cells in the DG showed a significant effect of UAG the main effect of treatment $(P=0.0391, F(1,16)=5.047)$ and interaction between genotype and treatment $(\mathrm{P}=0.0343, \mathrm{~F}(1,16)=5.352)$ (Figure 5.2A, B). The Holm-Sidak multiple comparisions test indicated that in comparison to WT-Vehicle treatment mice, there was a significant decrease in neurone activation in the DG; WT-Vehicle vs WT-UAG P=0.0147, WT-Vehicle vs GOAT KO-Vehicle P=0.0377, WT-Vehicle vs GOAT KO-UAG (P=0.0377).

These data demonstrate that UAG treatment inhibits cell proliferation, immature neurone number and neurone activation in the neurogenic niche of the hippocampus. Similarly, GOAT KO results in a reduction in basal levels of neurogenesis.

5.3.1.1 Genetic knockout of GOAT or UAG treatment reduced the number of newborn immature neurones and new-born non-neuronal cells in the SGZ of the DG.

As these mice were administered BrdU, a thymidine analogue that incorporates into dividing cells, we investigated the effect of UAG treatment and GOAT KO on the production of new-born immature neurones (BrdU' $\mathrm{DCX}^{+}$) in the DG (Figure 5.2C, D). Data analysis showed that the absence of GOAT reduced the survival of new-born immature neurones (main effect of genotype, $\mathrm{P}=0.0171, \mathrm{~F}(1,18)=6.896$ ) (Figure 5.2D), and reduced the number of non-neuronal cells (BrdU+ DCX-); WT-Vehicle vs WT-UAG, P=0.0438, WTVehicle vs GOAT KO-Vehicle, $\mathrm{P}=0.0487$ (Figure 5.2E). 


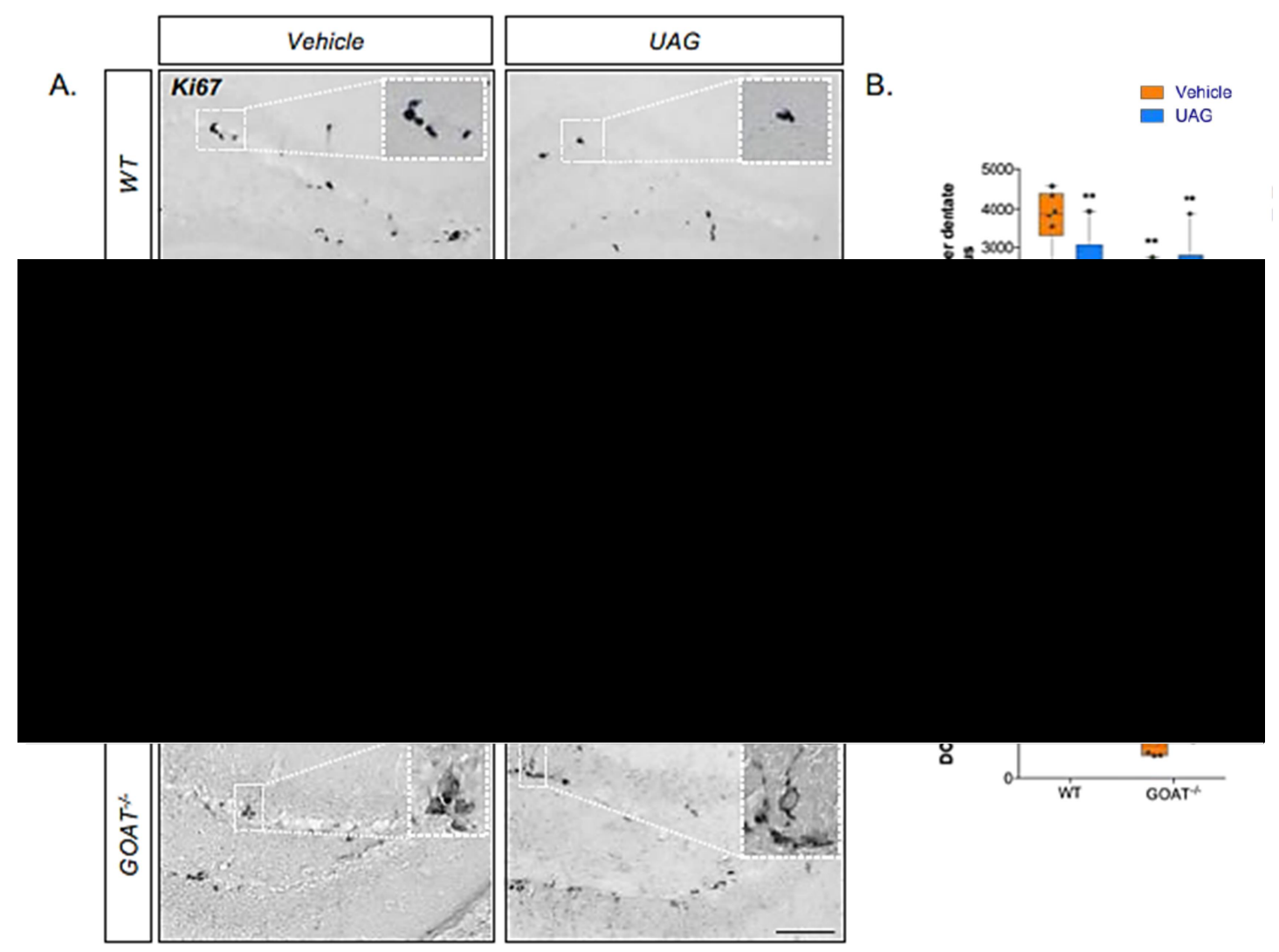

Figure 5.1 UAG treatment in WT and GOAT knockout mice impairs AHN.

Representative images of Ni-DAB staining of $\mathrm{Ki}^{+}(\mathrm{A})$ and $\mathrm{DCX}^{+}(\mathrm{C})$ cells in WT and GOAT-/- mice, that have been treated with vehicle or UAG. Reduction in the number of proliferating Ki67+ cells (B) and immature $\mathrm{DCX}^{+}$neuronal cells (D) in the DG. For statistical comparison a 2-way ANOVA with Holm-Sidak post-hoc analysis performed; ${ }^{*} P<0.05,{ }^{* *} P<0.01,{ }^{* * *} P<0.001$ vs WT-Vehicle. Data shown as mean \pm SEM. $\mathrm{n}=5-6$ mice/group. Main 10x magnification images, scale bar $=2100 \mu \mathrm{m}$, inset $40 \mathrm{x}$ images scale bar $=50 \mu \mathrm{m}$ (Hornsby et al., 2018). 


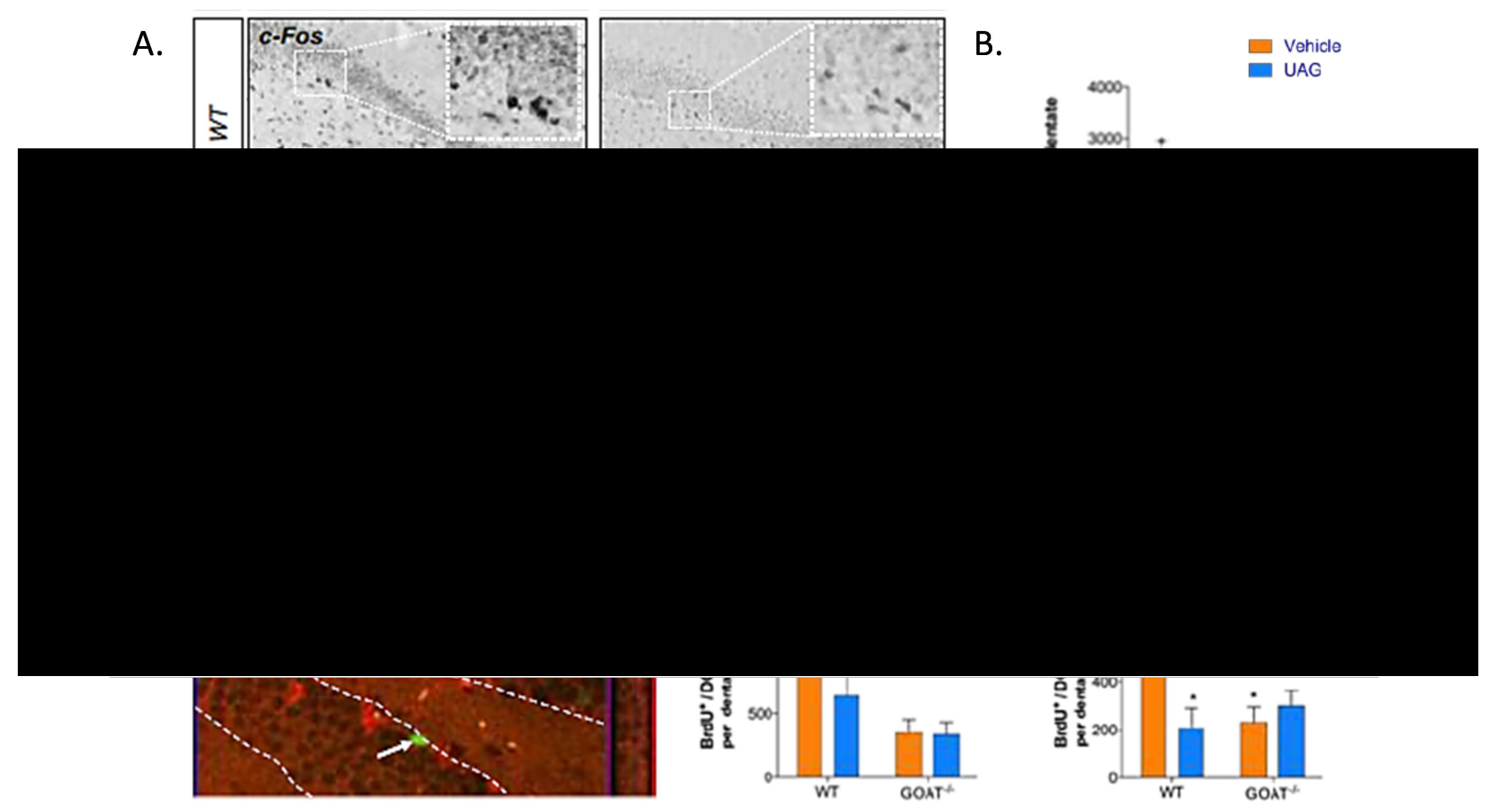

Figure 5.2 UAG treatment in WT and GOAT knockout mice impairs AHN continued.

Representative images of Ni-DAB staining of c-Fos ${ }^{+}(\mathrm{A})$ cells in WT and GOAT-/- mice, that have been treated with vehicle or UAG. Reduction in the number of neuronally active c-Fos ${ }^{+}$cells (B) in the DG. New BrdU+ ${ }^{+} C^{+}$immature neurone (arrowhead) and BrdU+DCX- (arrow)(C) non-neuronal cell survival rate was reduced by GOAT KO (D, E). For statistical comparison a 2-way ANOVA with Holm-Sidak post-hoc analysis performed; ${ }^{*} P<0.05,{ }^{* *} P<0.01,{ }^{* * *} P<0.001$ vs WT-Vehicle. Data shown as mean \pm SEM. $\mathrm{n}=5-6$ mice/group. Main 10x magnification images, scale bar $=2100 \mu \mathrm{m}$, inset $40 \mathrm{x}$ images scale bar $=50 \mu \mathrm{m}$ (Hornsby et al., 2018).

\subsubsection{GOAT-KO mice show no significant effect on the type II NSPC pool compared to WT controls.}

The type II Sox2 ${ }^{+}$NSPC pool was analysed, in order to confirm that genetic ablation of GOAT did not cause any anatomical changes to the DG of these mice, and whether the absence of GOAT affected NSPC numbers.

Analysis revealed no effect of genotype on Sox $2^{+}$cell number $(\mathrm{P}=$ 0.8865). No gross morphological changes to the DG were observed by the genetic ablation of GOAT (Figure 5.3). 


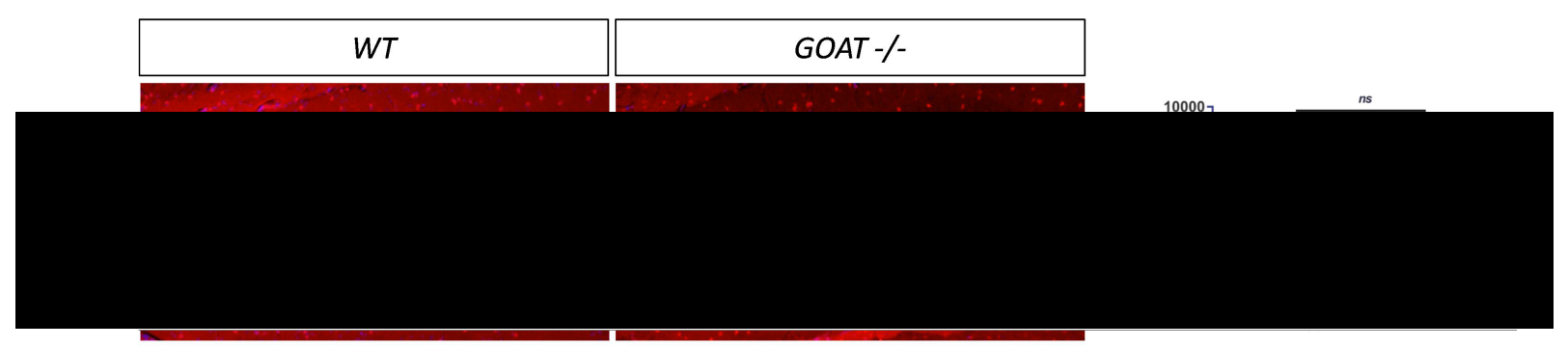

Figure 5.3 GOAT KO has no effect on type II NSPC number or structure of the DG.

Representative fluorescent images of Sox $2^{+}$cells in the DG of the hippocampus, of WT and GOAT KO mice. Sox $2^{+}$cells (red), Hoechst nuclear counterstain (blue), scalebar $=100 \mu \mathrm{m}$. Statisitical analysis performed, 2-tailed unpaired $t$-test, $\mathrm{n}=4$ /group, $\mathrm{ns}=$ no significance.

\subsubsection{A second GOAT KO model shows deficits in AHN, but not type II NSPC number.}

A second GOAT KO mouse model was also used (see 4.2.1.2) to quantify any effects on AHN. Hippocampal brain sections from 12-week old male mice were immunohistochemically stained for markers of cell proliferation $\left(\mathrm{Ki}^{+} 7^{+}\right.$, Figure 5.4A) and immature neurone production (BCX', Figure 5.4C). Analysis showed a decrease in these cell populations in the GOAT KO $(n=6)$ compared to WT controls $(n=6)$, however it was not significant. Statistical analysis of the dividing cell population $\left(\mathrm{Ki}^{+} 7^{+}\right)(2$-tailed unpaired $t$-test) showed that the reduction did not reach significance (WT vs GOAT KO P $=0.0602$ ), but indicated a deficit in the KO mice (Figure 5.4B). The immature neurone population $\left(\mathrm{DCX}^{+}\right)$was significantly reduced (WT vs GOAT KO P $=0.0234$ ) (Figure 5.4D). Similar to the other GOAT KO mouse model used (Taconic farms), these KO mice had no change in the type II NSPC pool $(\mathrm{P}=0.9770$, Figure 5.4E, F).

These data indicate that these GOAT KO mouse models have deficits in AHN, but that it is not due to a disruption to the NSPC pool from which these cells arise. 


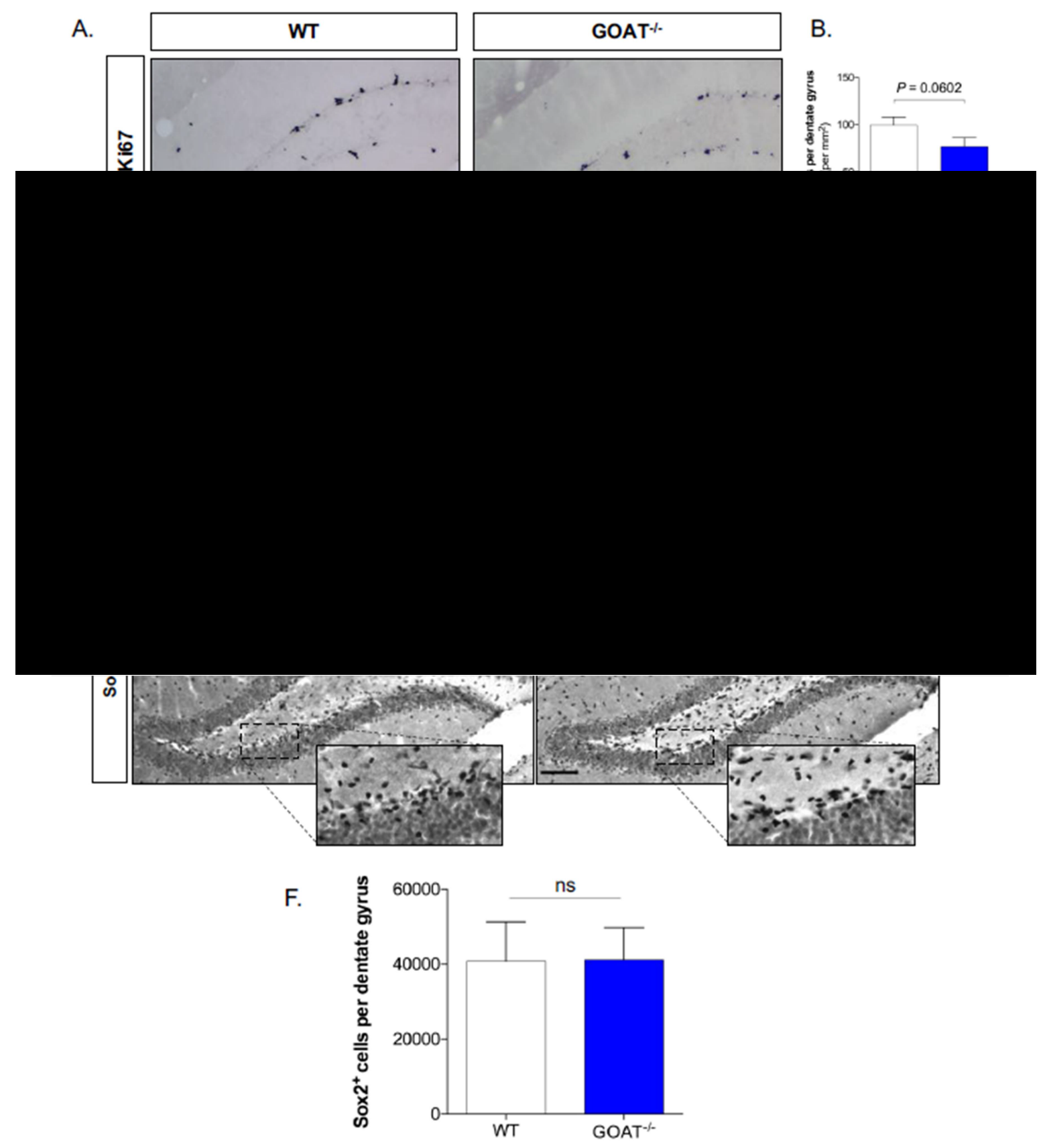

Figure 5.4 Regeneron (Yang et al, (2008) GOAT knockout mice have reduction in proliferating cells and immature neurone number, type II NSPC pool un-affected.

Reduction in cell proliferation (A, B) and neurogenesis (C, D) in the DG of GOAT KO mice compared to WT controls. Type II NSPC (Sox2+) number comparable between WT and GOAT KO mice (E, F). Statistical analysis performed; 2-tailed t-test. Scalebar $=100 \mu \mathrm{m} .{ }^{*} \mathrm{P}<0.05$. Data shown as mean \pm SEM. $\mathrm{n}$ $=6$ mice/group (Hornsby et al., 2018). 


\subsubsection{Immediate Early Gene (IEG) expression was unaffected in the DG}

following 7 day acyl-ghrelin treatment in WT and GOAT KO mice.

These WT and GOAT KO mice were treated with either vehicle (artificial cerebro-spinal fluid) or acyl-ghrelin for 7 days $(10 \mu \mathrm{g} / \mathrm{kg} /$ day i.c.v.), after which they were terminally anaesthetised. To determine whether acylghrelin treatment elicited a response in neuronal cell activation, in the presense and absence of the enzyme GOAT, the IEGs Egr-1 and c-Fos were analysed (Figure 5.5A, C).

Upon statistical analysis of the data (2-way ANOVA followed by Tukey's multiple comparison test) produced from immunohistochemical staining of the 2 targets, there was no significant change in expression of either IEG in the DG of the hippocampus (Figure 5.5B, D). There was no significant effect of genotype (Egr1, $\mathrm{P}=0.9890, \mathrm{~F}(1,12)=0.0001993$; c-Fos, $\mathrm{P}=$ 0.1247, $\mathrm{F}(1,18)=2.594)$, no effect of treatment $($ Egr1, $\mathrm{P}=0.2178, \mathrm{~F}(1,12)=$ 1.691; c-Fos, $\mathrm{P}=0.8237, \mathrm{~F}(1,18)=0.05112)$, and no effect on interaction of genotype and treatment $($ Egr1, $\mathrm{P}=0.3571, \mathrm{~F}(1,12)=0.9172 ; \mathrm{c}-\mathrm{Fos}, \mathrm{P}=0.6711$, $\mathrm{F}(1,18)=0.1863)$.

This indicates that the absence of GOAT does not affect neuronal cell activation, and there are no significant effects of acyl-ghrelin treatment on IEG cell number in this GOAT KO model (Regeneron). 


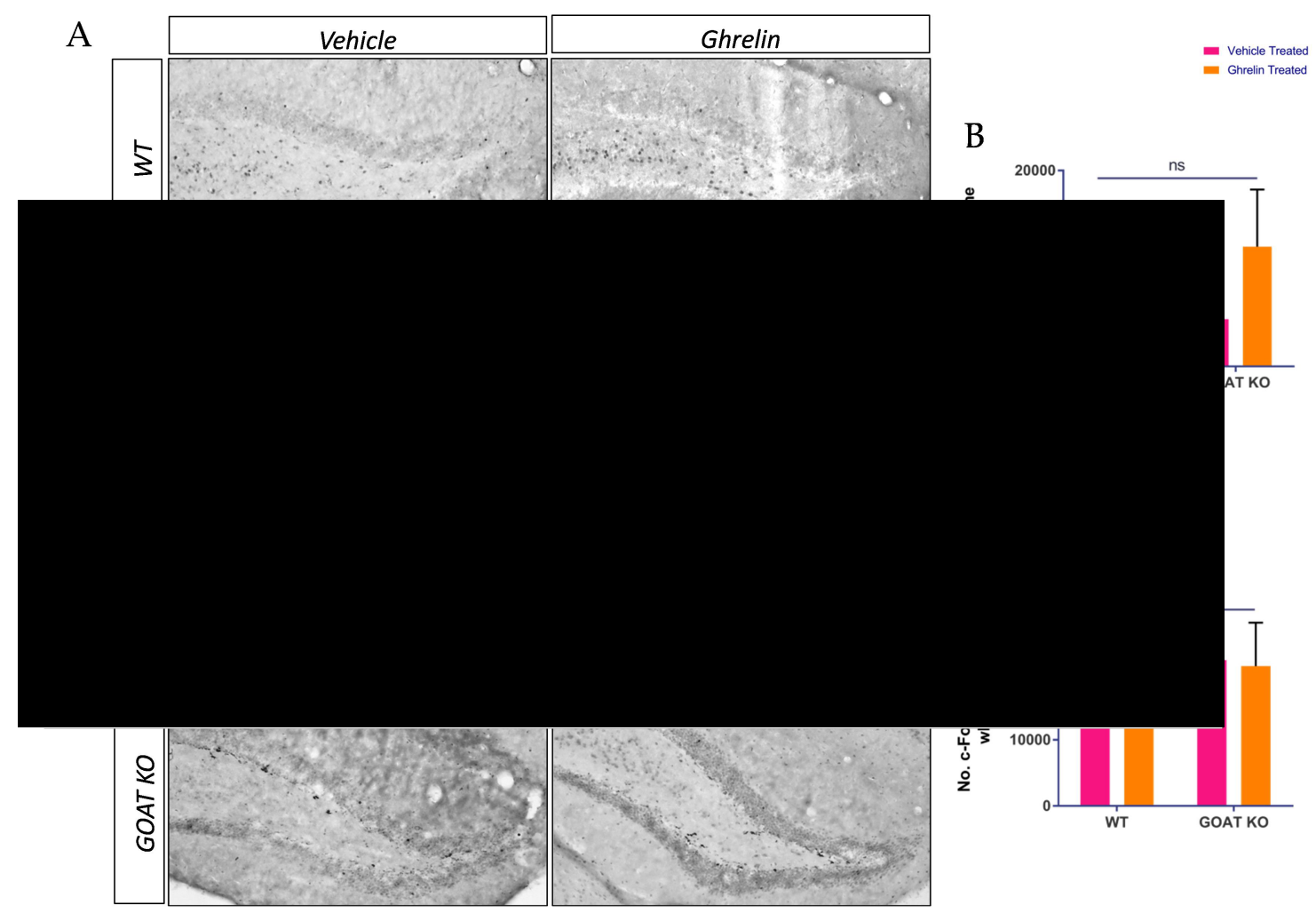

Figure 5.5 IEG expression is un-affected by genotype or AG treatment in the Regeneron GOAT knockout mouse model.

Representative images of Ni-DAB staining of IEGs Egr1 ${ }^{+}$(A) and c-Fos ${ }^{+}$cells (C). Scalebar $=100 \mu \mathrm{m}$. Data is expressed as mean $\pm \mathrm{SEM}, \mathrm{n}=4-8$ mice/group. Statisitical analysis performed; 2-way ANOVA followed by Tukey's multiple comparisons test. ns = no significance.

\subsubsection{Genotype has no overall effect on dendritic spine formation.}

The brain tissue from WT and GOAT KO mice were impregnated with Golgi-Cox solution to allow for the analysis of dendritic spines (Figure 5.6). An $n$ of 3 was used for each group and the different types of spines were counted on randomly chosen dendrites in the hippocampus. Statistical analysis using a 2-way ANOVA followed by a Fisher's LSD multiple comparisons test, revealed no effect of genotype $(P=0.1276, F(1,20)=2.526)$, however there does appear to be a significant reduction in the number of stubby spines in the GOAT KO mice compared to WT controls ( $\mathrm{P}=0.0164$, Figure 5.6B). There was an overall effect on spine type $(\mathrm{P}<0.0001, \mathrm{~F}(4,20)=$ 
26.36), but no significance in the interaction of genotype and spine type $(\mathrm{P}=$ $0.2327, \mathrm{~F}(4,20)=1.526)$.

These data indicate that the overall number of spines situated on hippocampal dendrites was not affected by genotype, but that the specific stubby spine type was significantly reduced in the absence of GOAT.

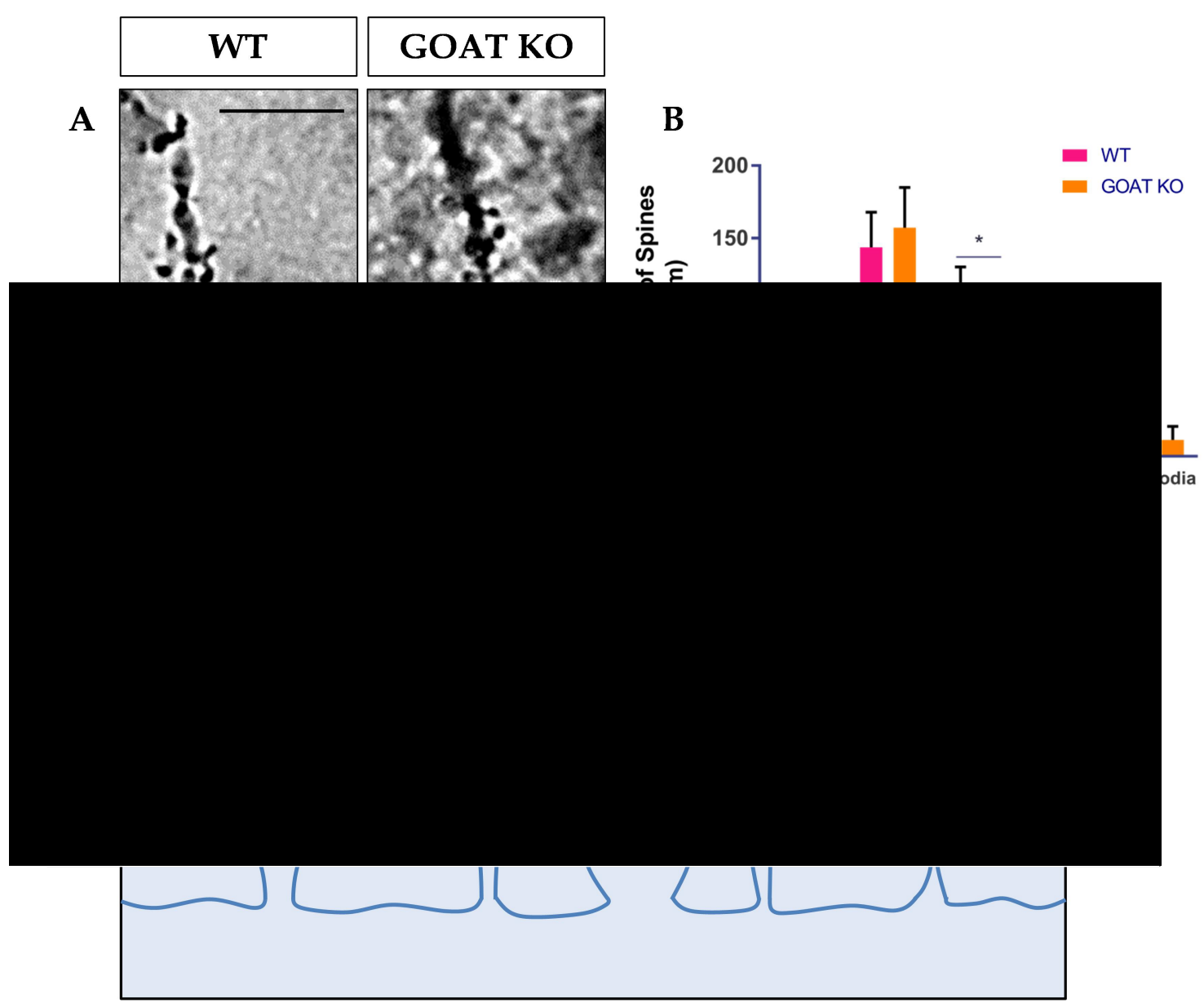

Figure 5.6 GOAT knockout significantly decreased the number of Stubby dendritic spines.

Representative images of dendrites stained with Golgi-cox, used for the analysis of different spine types (A). Scalebar $=10 \mu \mathrm{m} . \mathrm{n}=3$ /group. No overall effect of genotype, but a significant decrease in stubby spine number in GOAT KO mice (B). Schematic of dendritic spine types (C). Statistical analysis performed 2-way ANOVA and Fishers LSD post-hoc multiple comparisons test. Data displayed as mean \pm SEM. 


\subsubsection{Period of time post-acyl-ghrelin treatment significantly affects rates of new neuronal cell production.}

Two sets of WT and GOAT KO mice were administered vehicle (artificial cerebro-spinal fluid) and BrdU or acyl-ghrelin and BrdU for 7 days. After 7 days of treatment one group were terminally anaesthetised for tissue collection on day 8, whilst the other group were maintained until day 28 . This BrdU pulse-chase approach allowed analysis of new neurone survival/maturation via immunofluorescent staining and quantification of $\mathrm{BrdU}^{+} / \mathrm{NeuN}^{+}$on day 28, 21 days following the end of treatment (Figure 5.7).

Mice culled on day 8 showed no significant effect on new mature neurone survival/maturation (Figure 5.7A), no effect of genotype $(\mathrm{P}=$ $0.1456, \mathrm{~F}(1,12)=2.422)$, treatment $(\mathrm{P}=0.2334, \mathrm{~F}(1,12)=1.575)$ or interaction $(P=0.5073, F(1,12)=0.4672)$. In the population of new-born cells that are non-neuronal (Figure 5.7B), there appears to be a significant negative effect of treatment $(\mathrm{P}=0.0310, \mathrm{~F} 91,12)=5.97)$ in both WT and GOAT KO (genotype, $\mathrm{P}=0.4495, \mathrm{~F}(1,12)=0.6112)$. This indicates that the 7 day treatment of acyl-ghrelin is negatively impacting the production of new adult-born non-neuronal cells in the DG.

The group of WT and GOAT KO mice that were maintained until day 28, 21 days after treatment, appear to show the opposite effect. New adultborn mature neurone survival is positively modulated by acyl-ghrelin treatment $(\mathrm{P}=0.0237, \mathrm{~F}(1,14)=6.438)$ in both WT and GOAT KO mice (genotype, $\mathrm{P}=0.9263, \mathrm{~F}(1,14)=0.008863$ ) (Figure 5.7C), but the subset of new-born cells that are not neurones are unaffected by treatment $(\mathrm{P}=0.1443$, $F(1,15)=2.373)$ and genotype $(P=0.8135, F(1,15)=0.05766)($ Figure 5.7D).

This demonstrates that acyl-ghrelin treatment has a positive effect on $\mathrm{AHN}$ in the DG, with the presence and absence of GOAT at 28 days. 


\section{Short term analysis (Day 8)}

A

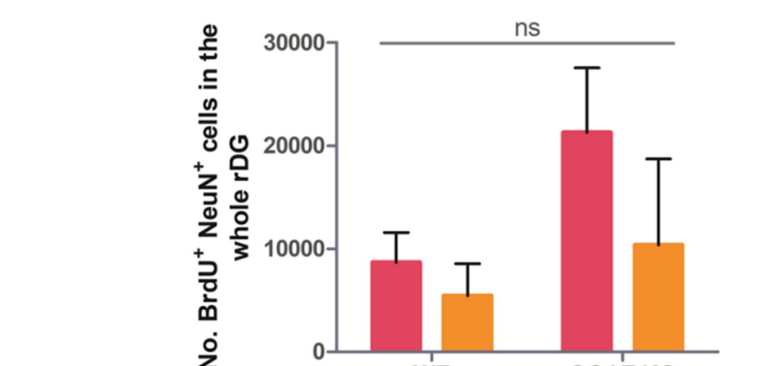

B

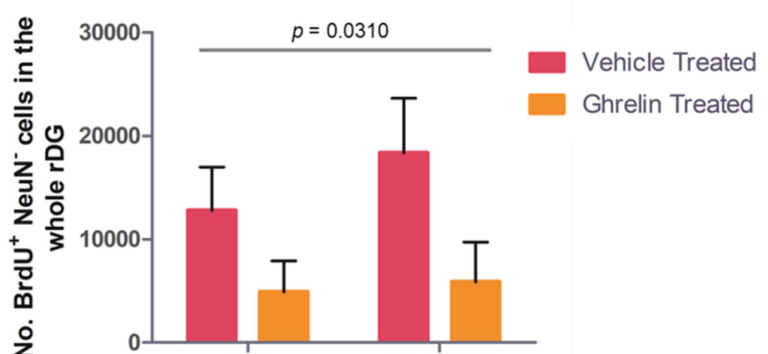

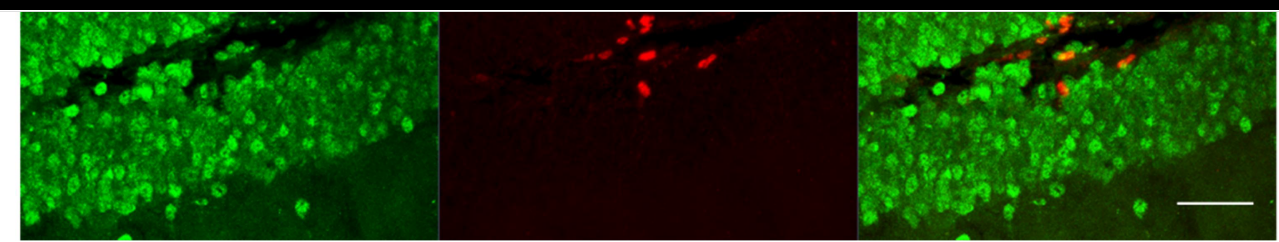

Figure 5.77 days of AG treatment appears to reduce new neuronal cell number, however after 28 days new neuronal cell number increases.

Short term (day 8) analysis of Acyl-ghrelin treatment has no significant effect on new mature neurone survival $\left(\mathrm{BrdU}^{+} \mathrm{NeuN}^{+}\right)$(A) but significantly reduces new non-neuronal cell (BrdU' $\left.\mathrm{NeuN}^{-}\right)$(B) irrespective of genotype. Mice sacrificed on day 28 show a significant increase in $\mathrm{BrdU}^{+} \mathrm{NeuN}^{+}$cells following acyl-ghrelin treatment (C), with no overall effect on BrdU+ NeuN- cells (D). Representative confocal image of $\mathrm{BrdU}^{+} \mathrm{NeuN}^{+}$(arrowheads) and BrdU+ $\mathrm{NeuN}^{-}$cells in the SGZ of the DG (E). Scalebar $=50 \mu \mathrm{m} . \mathrm{n}=4-8 \mathrm{mice} /$ group. Statistical analysis performed, 2-way ANOVA followed by Tukey's post-hoc multiple comparisons test. Data displayed as mean \pm SEM. 


\subsubsection{UAG treatment of WT mice does not affect AHN.}

12 week old WT C57BL/6 mice were subcutaneously administered saline or UAG $(80 \mu \mathrm{g} / \mathrm{kg} /$ day) for 7 days, to see if UAG treatment would have a negative effect on AHN.

Immunofluorescent staining of hippocampal DG sections for newborn mature neurones $\left(\mathrm{BrdU}^{+} / \mathrm{NeuN}^{+}\right)$(Figure 5.8) revealed that UAG treatment had no effect on $\mathrm{BrdU}^{+} / \mathrm{NeuN}^{+}$cell number in the DG $(\mathrm{P}=0.1831)$ and also had no effect on the sub-set of new-born cells that were nonneuronal $\left(\mathrm{BrdU}^{+} / \mathrm{NeuN}^{-}, \mathrm{P}=0.7179\right)$.

These data suggest that short-term UAG treatment has no effect on AHN. 


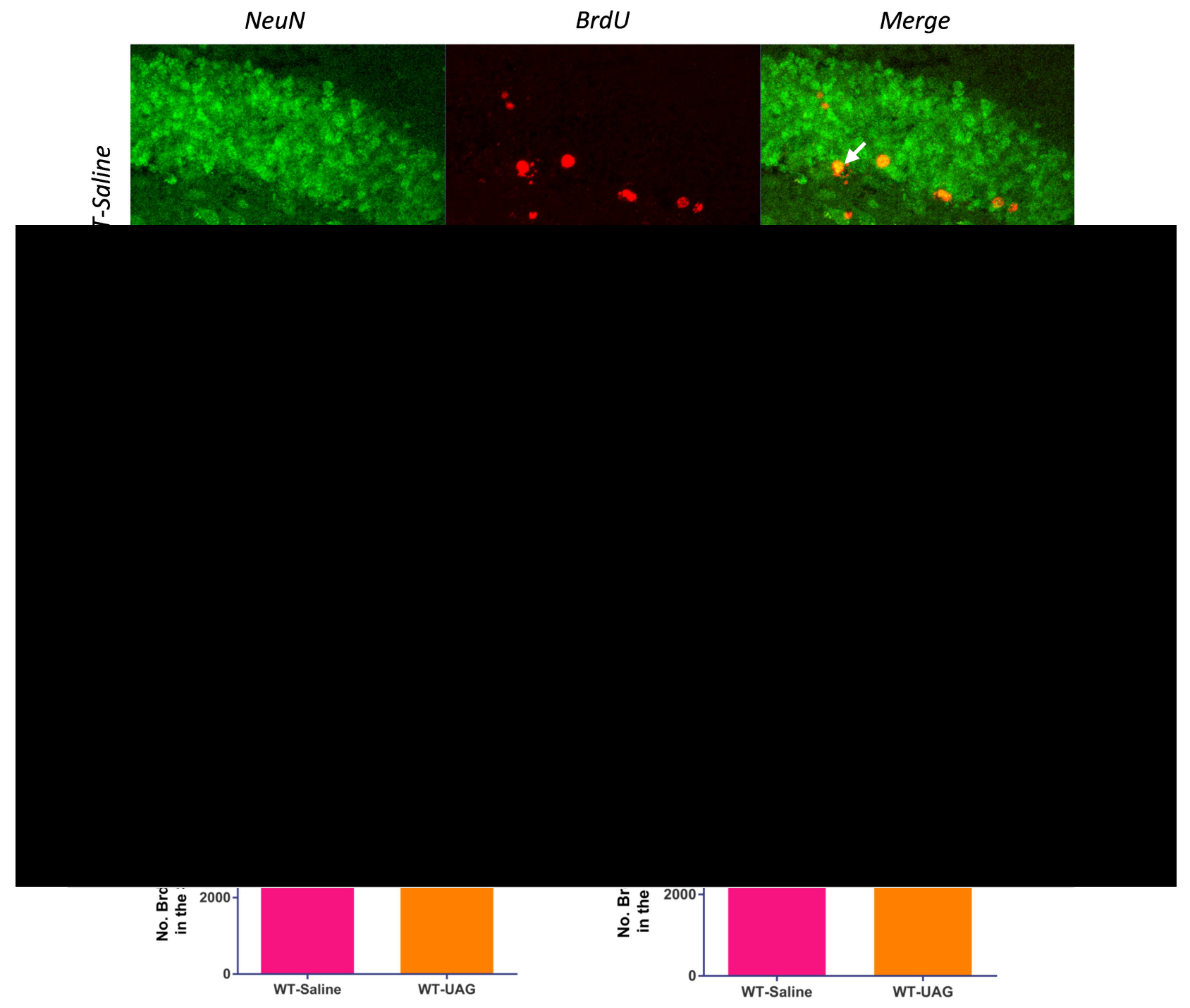

Figure 5.8 UAG treatment in WT C57BL/6 mice has no effect on the number of new adult-born neurones.

Representative confocal images of $\mathrm{NeuN}^{+}$(green), $\mathrm{BrdU}^{+}$(red) and $\mathrm{BrdU}^{+} \mathrm{NeuN}^{+}$(yellow, arrow) cells in the SGZ of the DG, in WT-saline and WT-UAG treated mice. $n=9$ /group, statistical analysis performed; unpaired 2-tailed t-test, $n s=$ no significance. Data represented as mean $\pm \mathrm{SEM}$. Scalebar $=$ $50 \mu \mathrm{m}$. 


\subsection{Discussion}

Acyl-ghrelin has been shown to have pro-neurogenic effects, promote synaptic plasticity and AHN (Li, Kim, et al., 2013). However, the role of the unacylated form, UAG, appears to be a little unclear. In this chapter, UAG is shown to negatively regulate neurogenesis in the hippocampus, but not at the expense of the NSPC pool. Also, the genetic ablation of the acylating enzyme, GOAT, which results in increased levels of circulating UAG and no AG, had a negative effect on AHN.

The 6-month old WT and GOAT KO mice from Taconic labs that received UAG infusion for 7 days had a significant reduction in cell proliferation $\left(\mathrm{Ki}^{+} \mathrm{7}^{+}\right)$, the number of immature neurones $\left(\mathrm{DCX}^{+}\right)$and cells that were active (c-Fos $\left.{ }^{+}\right)$in WT mice. GOAT KOs had similar reductions which weren't exacerbated by UAG treatment. There was also a significant reduction in the number of new adult-born immature neurones $\left(\mathrm{BrdU}^{+}\right.$ $\mathrm{DCX}^{+}$) in the DG following UAG treatment in WT mice and in GOAT KOs, compared to vehicle treated WT controls. Similarly, the Regeneron 12-week old GOAT KO mice also showed deficits in cell proliferation and immature neurone number, but no effect on cell activation. Both models had intact NSPC pools, that were unaffected by the increase in circulating UAG and absence of AG. UAG is thought to be the inactive form of ghrelin (Masayasu Kojima, Hosoda, Matsuo, \& Kangawa, 2001). However, there is increasing evidence of UAG having an antagonistic function to AG. For example, it prevents AG effects on secretion of insulin and glucose levels in human blood serum and plasma (Broglio et al., 2004), it reduces food intake that AG promotes in ddy mice who were i.p. injected with UAG $(1-3 \mathrm{nmol}, \mathrm{n}=15)$ (Asakawa et al., 2005), inhibits the output of glucose from porcine hepatocytes that is promoted by AG (Gauna et al., 2005) and quashes the effect of the GH-IGF1 axis by reduced serum levels in transgenic mice that overexpress UAG who also had a smaller phenotype (Ariyasu et al., 2005). In another study in GOAT KO mice and WT littermates (8-week old, male) that 
were $60 \%$ CR for 1 week in order to lose body fat, and then fasted for 23 hours. Following the fast, WT mice showed increases in GH levels, blood glucose, an increase in the marker of autophagy LC3II and number of autophagosomes in the liver, whereas GOAT KO mice were hypoglycaemic and reduced autophagy. This was reversed to WT levels upon the administration of GH $(15 \mu \mathrm{g} / 24 \mathrm{~h})$ to the GOAT KO mice (Y. Zhang, Fang, Goldstein, Brown, \& Zhao, 2015). All this bolsters the findings in GOAT KO models that increased levels of circulating UAG is causing a decline in aspects of neurogenesis and synaptic plasticity in the hippocampus, not just the absence of AG.

Where these two GOAT KO models differ is in their effects on newborn neurone production and cell activation (IEG expression). The Taconic labs mouse model exhibits a reduction in c-Fos expression in the GOAT KO compared to its WT controls, whereas no effect on Egr-1 or c-Fos was observed in the Regeneron model. This model also showed no effect of UAG treatment on new-born neurone production. These differences may be due to the difference in age of the mice used in each experiment. The Taconic labs model were 6 months old and may have shown more advanced impairment, whereas the Regeneron model mice were younger 12 weeks old. Dosage of UAG given, and the method in which it was administered to the Taconic labs model was a more physiological dose of $48 \mu \mathrm{g} /$ day via a jugular vein catheter which allowed the infusion to enter the brain through regular circulation. In contrast, the Regeneron model was administered a much higher dosage of $80 \mu \mathrm{g} / \mathrm{kg} /$ day via a more direct method of i.c.v. infusion. The method of delivery of BrdU may have had an impact on the numbers of new-born cells,

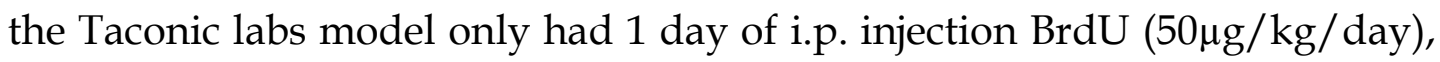
whereas the Regeneron model underwent a 7 day infusion of BrdU $(50 \mu \mathrm{g} / \mathrm{kg})$ via subcutaneous osmotic mini-pump. Another difference between the 2 models is in way they were generated. The Taconic lbs model is a complete genetic ablation of GOAT, by deletion of the gene MBOAT4 that codes for GOAT, whereas the Regeneron model uses Velocigene 
technology that uses a bacterial artificial chromosome (BAC) vector to replace the coding region of GOAT with a LacZ reporter gene containing cassette and Neomycon resistance gene, done in mouse embryonic stem cells, that are then injected in C57BL/6 blastocysts. These differences may have had a severe effect on the way that UAG performed within the brain.

In this final part of the study, brains from 12-week old WT and GOAT KO mice (Regeneron) were processed using Golgi-cox solution to dendritic spine number. It has previously been show that ghrelin promotes dendritic spine generation and reorganisation in the rat hippocampus (Berrout \& Isokawa, 2012). Analysis demonstrated that compared to WT, these GOAT $\mathrm{KO}$ mice showed no significant change in the total number of the various spine types. However, there is a 53\% reduction in the "stubby" spine in GOAT KO mice. These "stubby" spines are described as being an immature form of spine (Harris et al., 1992), suggesting that there may be a negative effect on spine maturation in the presence of higher UAG levels.

Another group of the Regeneron mouse model were administered AG $(10 \mu \mathrm{g} / \mathrm{kg} /$ day) for 7 days and sacrificed the following day or allowed to live for a further 21 days, and sacrificed on day 28. This was to determine whether AG's positive action on AHN would be affected by the absence of GOAT or by the presence of higher UAG levels. No significant effect was observed in new adult-born neurone production (BrdU ${ }^{+} \mathrm{NeuN}^{+}$cells) following the 7 days of AG treatment, however, 21 days following on from the AG treatment AHN was increased significantly $(P=0.0237)$ (compared the WT vehicle treated) independent of the presence of GOAT. These data suggest that AG's pro-neurogenic effects were not attenuated by increased UAG levels in GOAT KO mice and that the effects were observed over the longer term. This suggests that AG is promoting neuronal differentiation and/or the survival of new neurones, rather than promoting proliferation.

GOAT is necessary for the acylation of $A G$, not only does it perform this acylation in the stomach, but it is able to perform this locally in the 
hippocampus. This has been shown in a study by Mohammad Murtuza and Masako Isokawa, where immunohistochemical staining demonstrated GOAT immunoreactivity (IR) in the hippocampal DG of WT Sprague-Dawley rats and C57BL/ 6 mice and expression was unchanged between controls and 18 hour fasted animals, which was not present in the GHSR1a KO of both rats and mice. Ghrelin IR was also demonstrated in the cell soma of DG cells, with GOAT IR around the cell soma. The same GOAT IR was seen in hippocampal slice cultures of both WT animals, and was absent in the GHSR1a KO's. They also demonstrated that GHSR1a was required for the production of GOAT in the hippocampus by incubating tissue slices in a GHSR1a antagonist overnight, WT antagonist treated slices show no difrerence to the control, whereas GHSR1a KO slices showed a significant decrease in GOAT IR compared to WT control. Local acylation was demonstrated in live hippocampal slices incubated overnight with fluorescent AG or UAG, and both showed intense fluorescent signals in WT but not in GHSR1a KO, indicating that UAG had bound to GHSR1a. Finally, they treated WT slices with GOAT inhibitor, followed by incubation in fluorescent AG or UAG, and saw that UAG incubated slices showed no binding of UAG, indicating that GOAT is active and is octanoylating UAG in the hippocampus (Murtuza \& Isokawa, 2018).

The ghrelin system also has a deacylating enzyme, acyl-protein thioesterase 1 (APT1) (Satou, Nishi, Yoh, Hattori, \& Sugimoto, 2010). This has also been shown to be located in the hippocampus of C57BL/ 6 mice by insitu hybridisation of APT1 mRNA, showing expression in the DG, CA1-CA3 and motor cortex. Immunohistochemical staining demonstrated that APT1 is present in apical dendrites in the DG (Tatro et al., 2013).

To conclude, this chapter has elucidated a previously unknown function of UAG in negatively modulating adult hippocampal neurogenesis 
and plasticity, and provides evidence of an essential role for GOAT in supporting AHN in the adult mouse brain. 


\section{Chapter 6 The Effect of Calorie Restriction on circulating AG-UAG in Healthy, Parkinson's Disease and Parkinson's Disease Dementia patients}

\subsection{Introduction}

Due to an ever increasing aged population, the prevalence of neurodegenerative disorders such as PD has increased. PD is characterised by the progressive loss of dopaminergic neurones in the substantia nigra pars compacta and intracellular a-synuclein aggregation. The most notable symptoms of PD are those that affect motor function; resting tremor, rigidity, bradykinesia and postural instability. The most common treatment for PD is L-DOPA that treats these motor dysfunctions and deep brain stimulation (Morgan, Rees, Andrews, \& Davies, 2018; Parkinson's, 2018). Non-motor symptoms associated with PD are olfactory dysfunction, dementia, depression, dysautonomia such as gastrointestinal dysfunction and disturbances to sleep (Ziemssen \& Reichmann, 2007). Much is known about the neuropathology of these disorders, but the causative mechanisms that underpin the neuronal loss and cognitive deficit associated with them still remain unknown. PD affects approximately 127,000 people in the UK, that's 1 in every 350 people (Parkinson's UK 2018).

The circulating orexigenic peptide, ghrelin, is neuroprotective in murine models of PD (Bayliss \& Andrews, 2013). Ghrelin promotes synaptic plasticity and improves spatial memory deficits in the mouse hippocampus (Hornsby et al., 2016; Kent et al., 2015) indicating that it is important in memory function. Mild CR is also known to confer neuroprotection in rat and non-human primate models of PD (Colman et al., 2014; Wenzhen Duan 
\& Mattson, 1999). Indeed, CR-mediated neuroprotection in the mouse MPTP neurotoxin-based model of PD is dependent upon ghrelin-AMPK signalling in dopamine neurones (Bayliss, Lemus, Stark, et al., 2016). Furthermore, the ghrelin response to fasting is reportedly impaired in PD patients, but only total ghrelin (TG) was analysed (M. M. Unger et al., 2011). A more recent study showed that TG and AG levels are reduced in PD (N. Song et al., 2017), but a diagnosis of dementia was an exclusion criteria, so these patients were not assessed.

Ghrelin is not the only affected factor, other circulating factors such as IL-6 and TNFa have been shown to be affected in PD patients that have nonmotor related symptoms (Lindqvist et al., 2012). Also, factors such as leptin, growth hormone (GH) and insulin-like growth factor-1 (IGF-1) are affected (Fiszer et al., 2010) and is associated with the symptom of weight loss in PD.

Interestingly, PD patients were significantly impaired on an object pattern separation task that required DG-dependent discrimination between similar pictures (Wesnes \& Burn, 2014; Michael A. Yassa \& Stark, 2011). These data suggest a possible reduction in hippocampal neurogenesis in PD, which is essential for pattern separation memory function in mice (Clelland et al., 2009). However, further analysis of this paradigm is needed in PD.

\subsubsection{Aim of the chapter}

We hypothesize that the acylated to un-acylated ghrelin (AG:UAG) ratio will be reduced in PD and PDD patients compared to healthy agematched controls. Also, based on the work in chapter 4 that indicated that increased UAG has an opposing effect on neurogenesis in mice, PD/PDD/PD-MCI (PD with Mild Cognitive Impairment) patients who display deficits in cognition will have reduced levels of circulating AG. 
This work is important as it could potentially lead to ghrelin becoming a new therapeutic target for neurodegenerative disorders and cognitive decline in humans. 


\subsection{Materials \& Methods}

\subsubsection{Study Design and Criteria}

This study was designed in order to determine whether AG is an appropriate biomarker for Parkinson's disease dementia (PDD), and to determine whether there is a difference in the dynamic ghrelin response in PD, PDD and age-matched controls, following an overnight fast and refeeding.

Levels of TG and AG were measured from blood plasma samples which were taken from approximately 60 participants divided into 4 groups (controls, PD, PD-MCI and PDD). As ghrelin does not act alone, but in synergy with multiple factors and hormones, the following analytes were also examined: insulin, leptin, PYY, GLP-1, IL-6, TNFa, IGF-1 and GH.

This study was performed in collaboration with the David Burn group situated at Newcastle University with appropriate ethical consent and intuitional approval in place.

\subsubsection{Participant criteria}

The participants of this study were of both sexes and between the ages of 60 and 85 and had to fit the following criteria (Table 6.1):

Parkinson 's disease (PD) - have a recognised diagnosis of idiopathic PD made by a neurologist and have normal cognitive function (Montreal Cognitive Assessment (MoCA) $\geq 26$ ) and no sign of functional impairment caused by cognitive decline.

Parkinson's disease dementia (PDD) - have a recognised diagnosis of idiopathic PD for more than 1 year made by a neurologist, meet the Movement 
Disorder Society task Force Criteria for PDD and have a MoCA <21/30. They must also show evidence of activities of daily living (ADLs) are affected on the Informant Questionnaire on Cognitive Decline in the Elderly (IQ-CODE) and carer interviews.

Age-matched Controls - show no symptoms or signs of dementia or disorders that affect movement.

Table 6.1. Demographic information of study participants.

\begin{tabular}{|c|c|c|c|c|}
\hline & $\begin{array}{l}\text { Healthy } \\
\text { Controls } \\
(n=20)\end{array}$ & $\begin{array}{l}\text { Parkinson's } \\
\text { disease } \\
(\mathrm{n}=20)\end{array}$ & $\begin{array}{l}\text { Parkinson's } \\
\text { disease - mild } \\
\text { cognitive } \\
\text { impairment } \\
(n=8)\end{array}$ & $\begin{array}{l}\text { Parkinson's } \\
\text { disease } \\
\text { dementia } \\
(n=8)\end{array}$ \\
\hline $\begin{array}{c}\text { Age } \\
(\text { mean } \pm \text { sd) }\end{array}$ & $74 \pm 6.28$ & $72.2 \pm 5.51$ & $74.31 \pm 6.01$ & $74.75 \pm 5.99$ \\
\hline Male (\%) & $55 \%$ & $55 \%$ & $56.2 \%$ & 87.5 \\
\hline $\begin{array}{c}\text { MoCA } \\
\text { (mean } \pm \text { sd) }\end{array}$ & $28.35 \pm 1.14$ & $27.55 \pm 1.47$ & $14.12 \pm 5.14$ & $14.12 \pm 5.14$ \\
\hline $\begin{array}{c}\text { BMI }\left(\mathrm{kg} / \mathrm{m}^{2}\right) \\
(\text { mean } \pm \mathrm{sd})\end{array}$ & $24.75 \pm 2.04$ & $25.31 \pm 2.82$ & $24.03 \pm 3.31$ & $24.41 \pm 3.20$ \\
\hline
\end{tabular}

\subsubsection{Test Visit}

Upon the test visit, participants were asked to arrive fasted and without having taken any of their usual PD medication. They were fitted with a peripheral venous cannula and blood taken to be able to measure fasting levels of the analytes. The participants were then given a $300 \mathrm{kcal}$ test meal typical of a British diet (1 slice of white toast with butter, 1 pot activia strawberry yoghurt, 1 shortbread biscuit, jam and a glass of water). Blood was then taken from the cannula at 5, 15, 30, 60, 120 and 180 minutes after the 
standardised meal and taking approximately $200 \mathrm{ml}$ blood from each participant. $120 \mathrm{ml}$ of water was also given at 60 and 120 minutes following the meal. At 180 minutes the participants were given an ad libitum meal consisting of a selection of pre-measured foods (1 medium banana, 1 chocolate mousse, 1 bag ready-salted crisps, grated cheddar cheese, tomato and mozzarella pasta bake and 1 large glass of water), they were allowed to eat as much as they liked and to take their medication.

\subsubsection{Blood collection and storage}

As the octanoyl group of AG is unstable, it needed to be protected upon blood collection. The protease inhibitor AEBSF (4-(2-Aminoethyl)benzenesulfonyl fluoreide, Cat No: A8456, Sigma Aldrich) was added to the plasma collected for TG, AG and PYY analysis.

Whole blood was collected into lavender Vacutainer® EDTA-plasma tubes (Cat No: 6450) up to a volume of $7 \mathrm{ml}$ per tube. The tubes were then inverted gently after collection to prevent coagulation. $1 / 3$ of the blood was transferred immediately to a sterile and cold centrifuge tube containing AEBSF $(2 \mathrm{mg} / \mathrm{ml})$ to prevent proteinase activity, and mixed gently. The remaining 2/3 fraction was used for analysis of insulin, leptin, PYY, IL-6, GLP-1 (active) and TNFa. The 2 separate fractions were then centrifuged at 2000xg for 15 minutes at $4^{\circ} \mathrm{C}$, resulting in 3 layers (top plasma layer, mid white layer of leukocytes and the bottom layer which contains red blood cells). The top layer of plasma was carefully transferred into sterile tubes ( $200 \mu \mathrm{l}$ aliquots), labelled with the date and patient I.D, and stored at $-80^{\circ} \mathrm{C}$.

Platelet-rich plasma was used for the analysis of IGF-1 and GH, this fraction of blood was spun at $1000 x g$ for 15 minutes at $4^{\circ} \mathrm{C}$, then aliquoted and stored as stated above. 


\subsubsection{Total Ghrelin ELISA}

For the analysis of total ghrelin (TG) levels, untreated plasma samples were analysed using the Human Ghrelin (Total) ELISA Kit (Cat. No. EZGRT$89 \mathrm{~K}$, Millipore). All reagents were brought to room temperature prior to performing the assay.

Firstly, the wells of the microtiter assay plate were washed with $300 \mu 1$ diluted wash buffer, this was decanted and the excess liquid removed by tapping the plate smartly on paper towels. This was done 3 times in total. Immediately after, $20 \mu \mathrm{l}$ of Matrix solution was added to the blank, standard and quality control wells, $30 \mu \mathrm{l}$ of assay buffer was added to the blank and sample wells, and $10 \mu \mathrm{l}$ of assay buffer was added to the standard and quality control wells. Next, $20 \mu \mathrm{l}$ of the ghrelin standards in ascending order of concentration were applied to the appropriate wells in duplicate and $20 \mu 1$ of the quality controls (QC1 and QC2) were also added to the appropriate wells in duplicate. Subsequently, $20 \mu 1$ of neat plasma sample was added to the sample wells in duplicate, and then $50 \mu$ l of the antibody solution mixture (1:1 mixture of capture and detection antibodies) was added to all wells of the microtiter plate. The plate was sealed with a plate sealer and placed on an orbital plate shaker (400-500rpm) for 2 hours at room temperature.

After the incubation the plate's contents were emptied and the plate was washed 3 times as described above. $100 \mu l$ of enzyme solution was added to all wells and the plate was re-sealed and incubated for 30 minutes at room temperature on the shaker. The plate's contents were decanted and the plate tapped over paper towels to remove any residual solution, the plate was then washed 6 times in the manner described above. Finally, $100 \mu l$ of substrate solution was added to all wells and the plate was sealed and placed on the shaker for 5-20 minutes until a blue colour had developed in the ghrelin standards. Subsequently, $100 \mu$ l of stop solution was added to the wells - the blue colour turned yellow due to acidification. The plate was analysed using 
a BMG Labtech POLARstar Omega, with absorbance read at 450nm and 590nm, within 5 minutes.

\subsubsection{Multiple analyte analysis with Milliplex system}

AEBSF treated plasma samples were analysed using the Milliplex MAP Kit- Human Metabolic Hormone Magnetic Bead Panel 96-well plate assay (Cat. No. HMHEMAG-34K, Millipore), with beads to the following targets: leptin, insulin, IL-6, TNFa, PYY, GLP-1 active and acyl ghrelin (termed active ghrelin by the Millipore assay). The plasma samples were taken out of $-80^{\circ} \mathrm{C}$ freezer storage and defrosted slowly at $4^{\circ} \mathrm{C}$.

Firstly, reagents were brought to room temperature (RT, 20-25 ${ }^{\circ} \mathrm{C}$ ) before use, and some reagents required preparation before use in the assay. Using the 96-well plate provided, $200 \mu$ l of Assay buffer was added to each well and the plate sealed and placed on a plate shaker (500-800 rpm) for 10 minutes at RT. The assay buffer was removed by inverting the plate and tapping it onto paper towels numerous times. Then $25 \mu \mathrm{l}$ of each Standard or Control was pipetted into the allocated wells, with assay buffer being used for the background (0pg/ml standard). $25 \mu l$ Assay buffer was then added to all of the sample wells, followed by $25 \mu \mathrm{l}$ of Serum Matrix solution to the background, standard and control wells. Next, $25 \mu \mathrm{l}$ of each plasma sample was added to the allocated wells (samples were added in duplicate), and finally $25 \mu 1$ of the mixed magnetic bead solution was added to each well. The plate sealed with a plate-sealer, wrapped in foil and placed on a plate shaker overnight (16-18 hours) at $4^{\circ} \mathrm{C}$.

After overnight incubation the plate's contents were removed and washed using the following method: as a solid plate was used, a handheld magnet was used for the wash steps. The plate was applied to the magnet and allowed to rest for 60 seconds to allow the settling of the magnetic beads. 
The well solutions were removed by emptying into a waste container and the plate tapped gently onto paper towels to remove any remaining liquid. The plate was separated from the magnet and $200 \mu l$ of wash buffer was added to all wells and the plate was shaken by hand for 30 seconds. The plate was then reattached to the magnet for 60 seconds to allow the beads to settle and the plate contents were removed as described before. This was repeated 3 times.

Subsequently, $50 \mu \mathrm{l}$ of detection antibody was added to each well of the 96-well plate, then sealed and covered in foil and placed on a plate shaker for 1 hour at RT. After this incubation the plate's contents were not removed, $50 \mu 1$ of Streptavidin-Phycoerythrin was added to each well and then resealed and covered for 30 minute incubation at RT on the plate shaker.

Following this incubation, the plate was washed 3 times as previously described. $100 \mu 1$ of Sheath Fluid (\#171000055, Bio-Rad) was added to all wells of the plate which was placed on the plate shaker for 5 minutes to resuspend the beads. Finally, the plate was analysed on a Bio-Rad Bio-Plex 200 system. Prior to each use of the Bio-Rad Bio-Plex 200 system, calibration was performed with the Bio-Plex Calibration kit (\#171203060, Bio-Rad) as instructed by the Bio-Plex Manager software.

\subsubsection{GH and IGF-1 ELISA's}

For the analysis of human IGF-1 and GH, platelet-rich plasma samples were analysed using Human IGF-1 DuoSet ELISA (cat. No. DY291, R\&D Systems), and Human GH DuoSet ELISA (Cat. No. DY1067, R\&D Systems), using half-volume Nunclon Microwell 96-well plates. All reagents were brought to room temperature before they were used.

The capture antibody was diluted to its working concentration using PBS and $100 \mu \mathrm{l}$ was used to coat each well of a 96-well plate, this was 
incubated overnight. The wells were aspirated and washed 3 times with wash buffer, filling each well with $200 \mu$ l of wash buffer and inverting the plate and tapping on paper towels to remove excess liquid. The plate was blocked by the addition of $150 \mu$ l of reagent diluent to each well and incubated for 1 hour at room temperature on a plate shaker (450rpm). Following this block, the plate was washed 3 times as described above.

Subsequently, $50 \mu \mathrm{l}$ of each plasma sample and $50 \mu \mathrm{l}$ of standards in reagent diluent were added in duplicate to the appropriate wells. The plate was sealed and incubated for 2 hours at room temperature on a plate shaker. Following this incubation the plate was washed as previously described, and $50 \mu 1$ of detection antibody was added to each well. The plate was sealed and incubated for 2 hours at room temperature on a plate shaker. Next, the contents of the wells were removed and the plate was washed as before. $50 \mu 1$ of Streptavidin-HRP was added to each well of the plate before it was sealed and protected from direct light and incubated for 20 minutes on the plate shaker. The plate was then washed as above. $50 \mu \mathrm{l}$ of substrate solution was added to each well; the plate was sealed, protected from direct light and incubated for 20 minutes on the plate shaker at room temperature. Finally, $50 \mu 1$ of stop solution was added to each well. The plate was then analysed using a BMG Labtech POLARstar Omega, with optical density read at 450nm and 570nm, immediately after the stop solution was applied.

\subsubsection{Data handling, Analysis and Statistics.}

The levels of circulating hormones associated with aspects of neurodegenerative disorders were analysed from the blood plasma of patients with PD $(n=20), \operatorname{PD}+M C I(n=8), \operatorname{PDD}(n=8)$ and age-matched controls $(n=20)$. After fasting overnight, plasma was collected before a calorie controlled meal, and at specified time points following the meal $(5,15$, 30, 60, 120 and 180 minutes). From the mean concentration $(\mathrm{pg} / \mathrm{ml})$ of each 
analyte, the data was plotted along a time course to determine whether any differences occurred in response to fasting and re-feeding. The area under the curve (AUC) was calculated and plotted in a separate graph. Statistical outliers in the data were removed using the ROUT method $(Q=10 \%)$, and any patients that had incomplete blood collection were excluded. Statistical analysis performed on the AUC data used the Kruskal-Wallis test, as you could not assumed normal distribution of the data and more than 3 groups were being compared, followed by Dunn's post-hoc multiple comparison test. 


\subsection{Results}

\subsubsection{The effects of fasting and re-feeding on circulating analytes in the blood plasma of PD and PDD patients, and age-matched controls.}

Analysis of total ghrelin (TG) levels has shown that there was no overall significance $(P=0.2788)$, and compared to age-matched controls there was no significant effect on TG levels in the PD (P > 0.9999), PD+MCI (P > 0.9999) or PDD $(\mathrm{P}=0.9290)$ groups (Figure 6.1A, B). For acyl-ghrelin $(\mathrm{AG})$ there was an overall significance between the groups $(\mathrm{P}=0.0094)$, and compared to age-matched controls there was no significant effect with PD (P > 0.9999), PD+MCI $(\mathrm{P}>0.9999)$ or PDD $(\mathrm{P}=0.1202)$ group. However, a significant difference between the PD and PDD group was observed $(\mathrm{P}=0.0055)$, suggesting that the significant decrease in AG levels is indicative of the presence of dementia (Figure 6.1C, D).

No overall significance was seen in the insulin response (Figure 6.1E, F) $(P=0.6055)$, and there was no significant difference between any of the groups compared to age-matched controls $(\mathrm{P}>0.9999)$. The leptin response (Figure 6.1G, H) appears reduced in AUC, but was not statistically significant overall $(\mathrm{P}=0.3717)$, with no significant differences between the PD and MD+MCI ( $\mathrm{P}>0.999)$, PDD group $(\mathrm{P}=0.5800)$ compared to controls, or between the PD and PDD group $(\mathrm{P}=0.6251)$.

No overall significance was observed in the gut hormone GLP-1 response $(\mathrm{P}=0.2814$, Figure $6.2 \mathrm{~A}, \mathrm{~B})$, and relative to controls there was no significant difference in PD $(\mathrm{P}>0.9999), \mathrm{PD}+\mathrm{MCI}(\mathrm{P}>0.9999)$ or the PDD group $(\mathrm{P}=0.60047460)$. Also, compared to the $\mathrm{PD}$ group there was no significant difference in PD+MCI $(\mathrm{P}>0.999)$ and PDD $(\mathrm{P}=0.5716)$. PYY (Figure 6.2C, D) was also statistically unaffected, although the graphs do 
indicate reduction in concentration compared to controls (overall $\mathrm{P}=0.4199$; control vs PD P > 0.9999, PD+MCI P > 0.9999 and PDD P = 0.6004).

The pro-inflammatory cytokines IL-6 (Figure 6.2E, F) and TNFa (Figure $6.2 \mathrm{G}, \mathrm{H})$ were also unaffected $(\mathrm{P}=0.1913 ; \mathrm{P}=0.1300)$ in their overall response, and relative to controls there was no significant difference with PD $(\mathrm{P}>0.999) \mathrm{PD}+\mathrm{MCI}(\mathrm{P}>0.9999 ; \mathrm{P}=0.1568)$ or $\mathrm{PDD}(\mathrm{P}>0.9999)$. These data indicate that the circulating inflammatory response is unaffected in individuals with PD.

Finally, circulating IGF-1 and GH was also unaffected (Figure 6.3A - D) $(\mathrm{P}=0.6605 ; \mathrm{P}=0.8202)$, with no significant differences in any of the groups (P > 0.9999) compared to age-matched controls. 

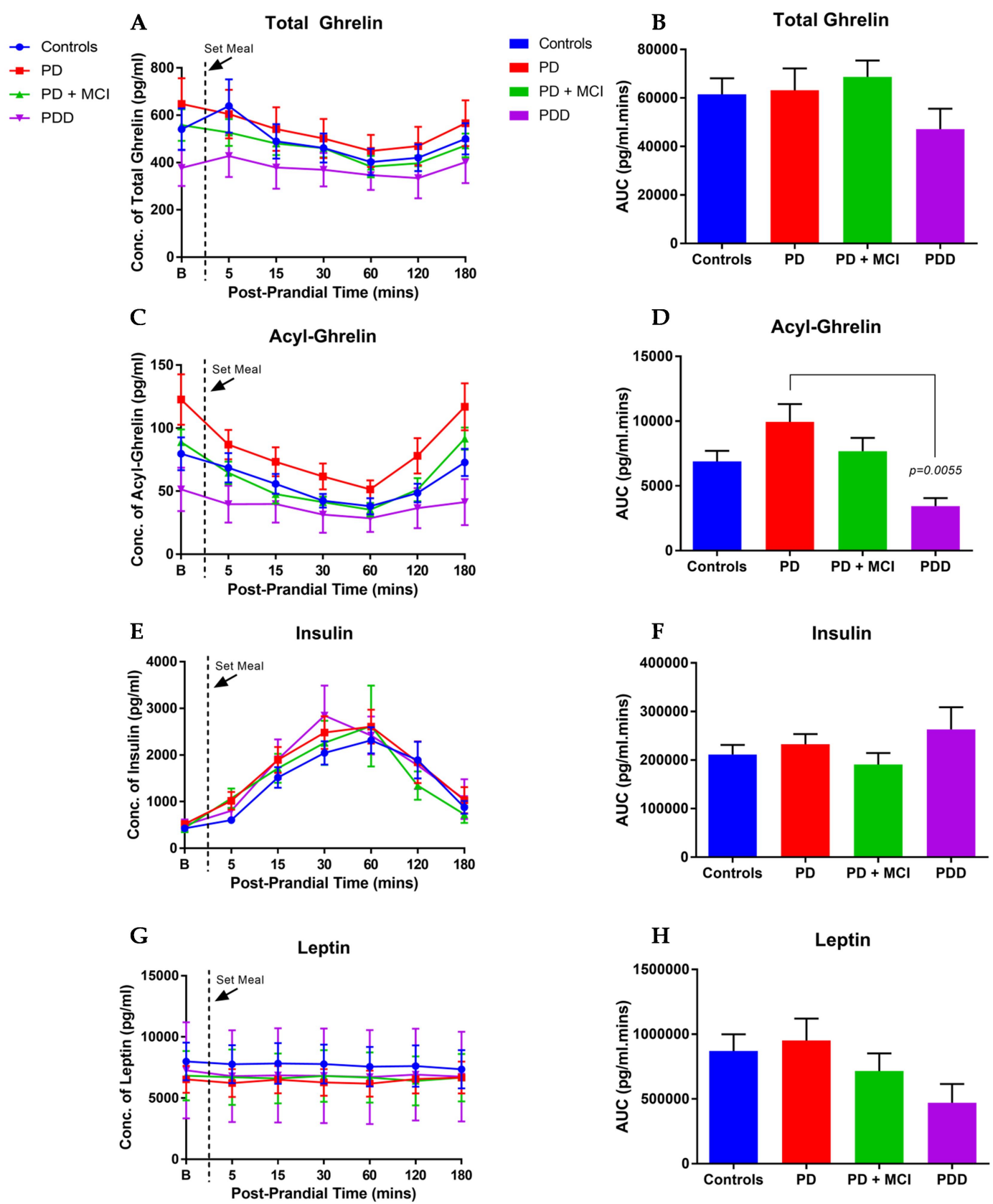

Figure 6.1 Timelines and Area Under Curve (AUC) for circulating plasma analytes.

Graphs demonstrating the effect of fasting and re-feeding response in circulating blood plasma analytes, and the AUC for each of the following analytes; Total Ghrelin (A, B), Acyl-ghrelin (C, D), Insulin (E, F) and Leptin (G, H). n = 20 (controls), 20 (PD), 8 (PD-MCI) and 8 (PDD). Statistical analysis performed on AUC, Kruskal-Wallis test and Dunn's post-hoc multiple comparisons test. Data represented as mean \pm SEM. 

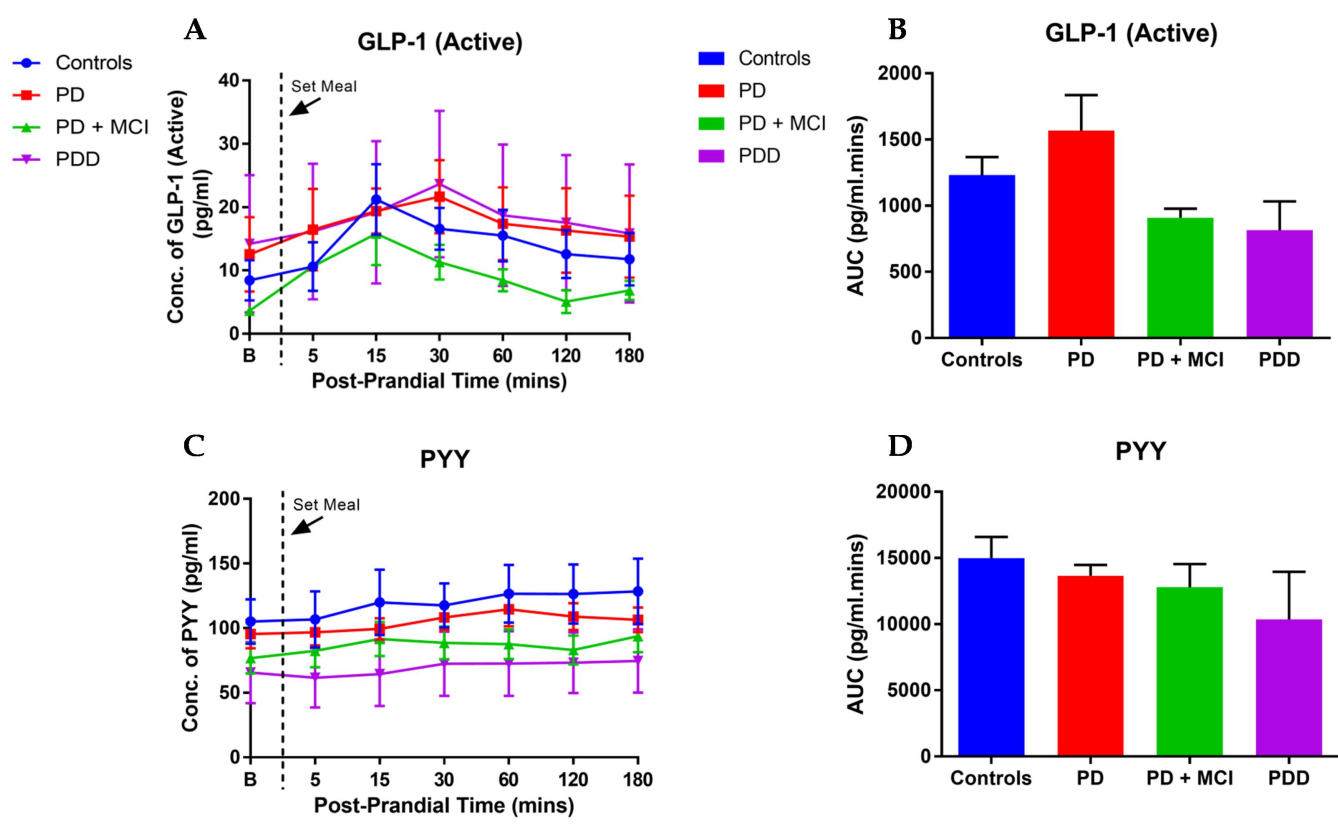

E

IL-6
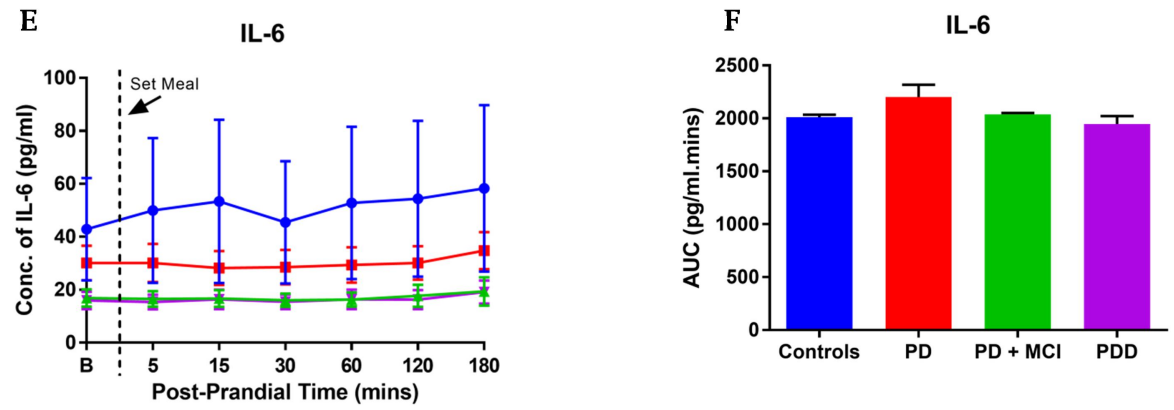

G

TNF $\alpha$

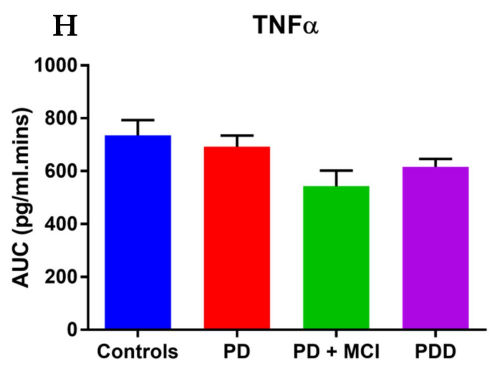

Figure 6.2 Timeline and AUC graphs continued.

Graphs demonstrating the effect of fasting and re-feeding response in circulating blood plasma analytes, and the AUC for each of the following analytes; GLP-1 (Active) (A, B), PYY (C, D), IL-6 (E, F) and TNFa $(\mathrm{G}, \mathrm{H}) . \mathrm{n}=20$ (controls), 20 (PD), 8 (PD-MCI) and 8 (PDD). Statistical analysis performed on AUC, Kruskal-Wallis test and Dunn's post-hoc multiple comparisons test. Data represented as mean \pm SEM. 

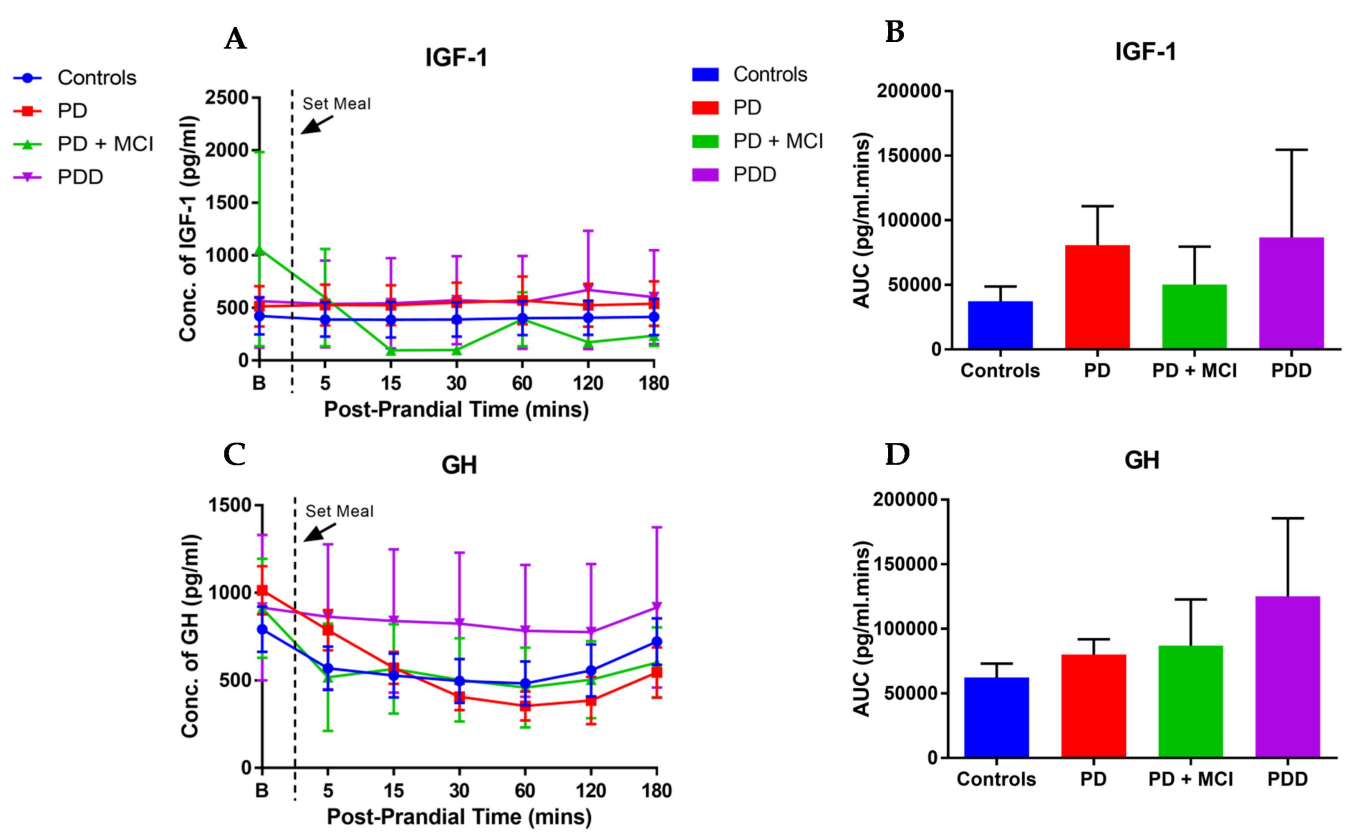

Figure 6.3 Timeline and AUC graphs continued.

Graphs demonstrating the effect of fasting and re-feeding response in circulating blood plasma analytes, and the AUC for each of the following analytes; IGF-1 (A, B) and GH (C, D), IL-6. n = 20 (controls), 20 (PD), 8 (PD-MCI) and 8 (PDD). Statistical analysis performed on AUC, Kruskal-Wallis test and Dunn's post-hoc multiple comparisons test. Data represented as mean \pm SEM.

\subsubsection{Fasting and re-feeding effects on the AG:UAG.}

AG plasma levels, as a percentage of TG (AG:TG ratio), in the fasted condition (baseline) was significantly reduced in patients with PDD, $\mathrm{P}=$ 0.0302 (Figure 6.4A) compared to age-matched controls. This difference was also detected in the post-prandial (180 minutes) levels of AG in the PDD cohort $(\mathrm{P}=0.0144)$ compared to controls (Figure 6.4B). These data suggest that PD patients diagnosed with dementia have significantly reduced circulating AG:TG ratio in comparison to PD patients who do not exhibit severe cognitive impairment or healthy individuals. This novel finding indicates that ghrelin may be a suitable biomarker for cognitive decline. 
A Fasting Acyl-Ghrelin (\% of Total Ghrelin)

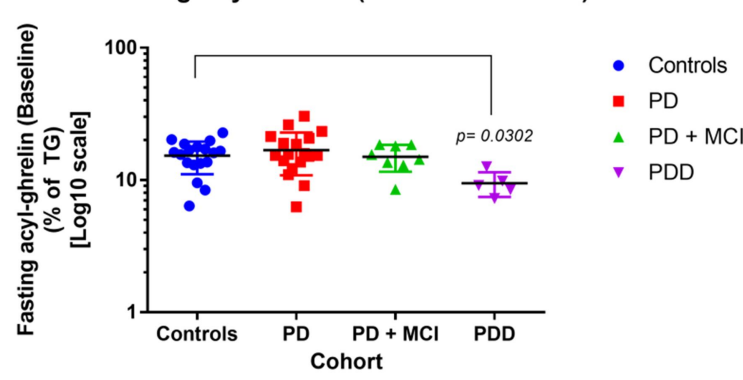

B Post-prandial Acyl-Ghrelin (\% of Total Ghrelin)

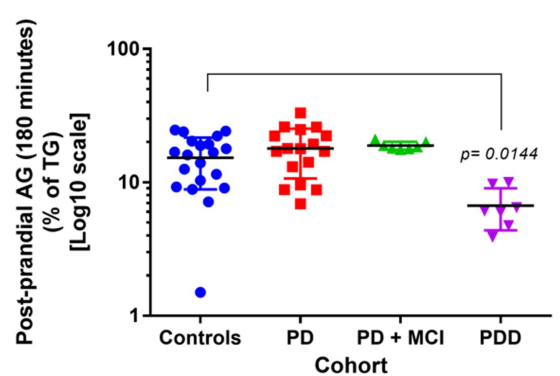

Figure 6.4 The fasted and post-prandial AG:UAG ratio is significantly reduced in PDD patients, compared to age-matched controls.

Circulating AG in healthy controls $(n=20), \operatorname{PD}(n=20), \operatorname{PD}-\mathrm{MCI}(n=8), \operatorname{PDD}(n=8)$, in the fasted (baseline) (A) and post-prandial (180 minutes) state (B). Statistical analysis performed by KruskalWallis test followed by Dunn's post-hoc multiple comparison test. 


\subsection{Discussion}

Current treatments for PD are targeted towards alleviating specific symptoms, such as L-DOPA for motor dysfunction. Calorie restriction promotes the survival of new-born NSPC's in the ageing brain of mice, which is important for mneumonic function (J.-H. Park et al., 2013), and improves cognitive function in other neurodegenerative disorders (Kang, Moon, Kim, Kim, \& Park, 2015).

At present, there is no pre-symptomatic treatment or preventative therapeutic measure in place. From this pilot study examining concentrations of circulating factors in the blood plasma, PD patients diagnosed with dementia have a reduced AG response compared to age-matched controls. They exhibited lower fasting and post-prandial AUC, showing an alteration in the usually dynamic ghrelin response to food.

PD patients suffer from reduced gut motility (M. M. Unger et al., 2010), which may also be an indication of a disruption to ghrelin action in PD (M. M. Unger et al., 2011). Gut dysfunction can occur early on in PD pathology before motor function impairments (Sung, Park, \& Kim, 2014), and include symptoms such as sialorrhea, dental disorders, dysphagia, gastroparesis, constipation and intestinal bacterial overgrowth. Gastric dysfunction is shown in $70-100 \%$ of PD patients with symptoms such as nausea, vomiting, weight loss, delayed gastric emptying, dehydration and malnutrition, which can lead to the malabsorption of drugs given to treat the disorder (Mukherjee, Biswas, \& Das, 2016). A recent study involving gut epithelial cells has shown that they may play a role in the transmission of PD pathology from the gut to the brain via the vagal nerve. The death of neurones in PD is characterised by the aggregation of mis-folded a-synuclein forming Lewy bodies in cell bodies and axons, and the movement dysfunction is caused by the death of neurones in the SNpc. This study showed that gut entero-endocrine cells (EEC) contain a-synuclein that forms 
connections with a-synuclein containing nerves, thereby forming a gut-brain neuronal circuit. a-Synuclein was detected in STC-1 cells (mouse intestinal cell line), CCK cells (mouse duodenal cells) and in enteric nerves in human duodenal tissue (Chandra, Hiniker, Kuo, Nussbaum, \& Liddle, 2017).

From the work in this chapter, concentrations of the circulating proinflammatory immune cytokines IL-6 and TNFa were unaffected in PD, $\mathrm{PD}+\mathrm{MCI}$ and PDD patients, compared to age-matched controls. Circulating immune factors such as TNFa and IL-6 have been linked with PD. For example, activation of microglia associated with central inflammation, results in the release of TNFa and IL-6 and other pro-inflammatory factors that contribute to nigral cell death via NFkB activated apoptosis. There is evidence that TNFa and IL-6 are elevated in the plasma and serum of PD patients (Su \& Federoff, 2014).

The analysis of leptin in this study showed no significant difference between any of the groups, although there was a reduction in AUC (Figure 5.1) in PDD. Another study showed that there was no correlation between serum leptin levels and PD and PD patients with lower cognition scores, compared to controls. They assessed blood serum levels of leptin in 30 healthy controls (mean age 58) and PD patients (mean age 59) after overnight fasting (Kenangil \& Ozdilek, 2016). Leptin (100nM) was neuroprotective in dopaminergic $\left(\mathrm{TH}^{+}\right)$midbrain primary neuronal cultures (C57BL/6 mice) following cell death induction with TNFa $(20 \mathrm{mg} / \mathrm{ml})$ and 6-OHDA $(25 \mu \mathrm{M})$ (Doherty, Oldreive, \& Harvey, 2008). Also, whilst no change was seen in the insulin response in this study, insulin has been linked to neuroprotection in human cell models of PD. A study used human SH-SY5Y (neuroblastoma) cells that had been differentiated into neurones with retinoic acid (SHSY5Y+RA) and exposed to $\mathrm{MPP}^{+}$(1-methyl-4-phenyl pyridinium) to induce neurone death. Pre-treated for 1 hour with insulin (100nM) before 24 hour treatment with or without $\mathrm{MPP}^{+}(500 \mu \mathrm{M})$, resulted in increased cell viability, and a reduction in nitric oxide and reactive oxygen species. Western blot 
analysis for TH (tyrosine hydroxylase) also demonstrated that insulin pretreatment was neuroprotective in these dopaminergic neurones. (Ramalingam \& Kim, 2015).

The analyte GLP-1 was not significantly altered in any of the groups studied, despite a decrease in the PD+MCI and PDD groups. The GLP-1 receptor is a drug target for diabetes, and the drug Exenatide has been shown to have potential as a therapeutic in PD. The lab of Tom Foltynie examined the effect of Exenatide treatment (twice daily subcutaneous injections for 12 months) on MDS-UPDRS (Movement Disorders Society Unified Parkinson's disease scale) and the MDR scale (Mattis Dementia Rating) in PD patients $(n=20)$ and controls $(n=24)$. They report that after 24 months (12 months following the final Exenatide injection) PD patients showed a 5.6 point improvement in MDS-UPDRS and a 1.8 point improvement in MDR scale compared to controls (Aviles-Olmos et al., 2014).

Biomarkers for the detection of early PD stages are essential for the development of new treatments and aid in earlier diagnosis of those most at risk of developing PD. Blood-based biomarkers are essential for PD diagnosis as non-motor symptoms can occur years before PD pathology becomes apparent. Potential blood-based biomarkers include; a-synuclein, the protein found in Lewy body lesions, which has been identified in plasma and cerebrospinal fluid (CSF); DJ-1 gene (PARK7), which is mutated in familial PD has also been detected in plasma and CSF, but multiple cohort studies have given conflicting outcomes of there being significant changes in PD compared to controls. Another potential biomarker is uric acid, as it has been found in the SNpc of PD patients and shown to contribute to DA neuronal loss due to increased oxidative stress (Chahine, Stern, \& Chen-Plotkin, 2014). Neuroimaging is a non-invasive form of biomarker, with the most commonly used being DaTSCAN, which images the dopamine system by targeting the dopamine transporter (DAT). A DAT ligand is administered by injection and it's binding ability is measured in the basal ganglia via SPECT (Single-photon 
emission computed tomography) imaging, where in PD cases you would observe reduced binding (Sherer, 2011). Notably, there have been recent developments in PET (positron emission tomography) imaging with the design and synthesis of fluorine-bearing Quinazolinone derivatives for the ghrelin receptor (GHSR1a). 2 fluorine-bearing compounds were found to have high binding affinity to GHSR1a, and are currently awaiting testing in in vivo models of murine cancer (Hou, Kovacs, Dhanvantari, \& Luyt, 2018). There are initiatives in place dedicated to the development of diagnostic biomarkers the Michael J. Fox Foundation Parkinson's Progression Markers Initiative (PPMI) and the National Institute of Neurological Disorders and Strokes (NINDS) initiative Parkinson's Disease Biomarkers Program (PDBP). These initiatives aim to standardize data collection protocols, support existing and new studies in PD patients, and help to develop the bioassays and tools for analysing bio-specimens and the data it produces (Chahine et al., 2014).

The data generated in this chapter has demonstrated that fasting and post-prandial levels of AG are significantly decreased in PDD compared to age-matched controls. Other neurodegenerative disorders such as frontotemporal lobar degeneration (FTLD) have been linked with increased levels of ghrelin (FTLD $n=69$ vs controls $n=88$ ) (Zanardini et al., 2017). In addition, in moderate $\mathrm{AD}$ there was no reported difference between basal ghrelin levels (overnight fast) in mixed gender age-matched controls $(n=23)$ and AD patients $(n=27)$. However, they did observe a significant decrease in ghrelin AUC in male AD patients compared to controls, indicating a potential sex difference. Therefore, the significant reduction in AG in PDD could be specific to this disorder.

AG has been shown to illicit neuroprotective effects in mouse models of PD. A study using GOAT KO mice, that lack circulating AG, treated with MPTP (1-methyl4-pheyl-1,2,3,6,tetrahydropyridine) after chronic AG injections, prevented dopaminergic neurone loss. Whereas, UAG had no 
neuroprotective effect on the number of dopaminergic neurones following MPTP treatment, and also increased levels of the stress hormone, corticosterone (Bayliss, Lemus, Santos, et al., 2016).

To conclude, the reduction in AG:UAG levels following fasting and post-prandially in PDD patients is a novel finding. To the best of our knowledge, no other study looking at levels of AG in PD has taken cognitive decline into account. This finding signifies the potential importance of AG and UAG as an indicator of cognitive dysfunction in neurodegenerative disorders. 


\section{Chapter 7 General Discussion}

\subsection{Summary}

The experiments in this thesis have led us to better understand the role of ghrelin in AHN. The work in chapter 3 investigated the expression of GHSR in extra-hypothalamic areas of the brain that are important for learning and memory i.e the DG, BLA, LEnt and CingC. Characterisation of the cells that express GHSR, within the hippocampal neurogenic niche of the DG, indicated that only mature dentate granule neurones $\left(\mathrm{NeuN}^{+}\right)$expressed GHSR. No expression was seen on dividing, type I or type II NSPC's, however the type II NSPC's were in close proximity to the GHSR-eGFP+ cells.

Following on from this, the experiments in chapter 4 allowed us to study the mechanism by which ghrelin mediates its beneficial effects on AHN. CR was used to observe the effects on AHN in mouse models lacking GHSR and circulating ghrelin. This work provided us with the novel finding that the positive effects on AHN mediated by $\mathrm{CR}$ are dependent on the presence of GHSR. Also, the increase in neuronal cell activation by CR and AG treatment was more robust in cells that did not express GHSR, leading to the assumption that there may be another factor involved in promoting AHN.

Chapter 5 experiments using GOAT KO mice and UAG treatment showed a unique role for UAG as a negative regulator of $\mathrm{AHN}$, by inhibiting cell proliferation and immature neurone production, as well as highlighting an important role for GOAT.

Finally, the pilot study performed in chapter 6, involved measuring concentrations of $\mathrm{TG}$ and $\mathrm{AG}$, along with other factors affected in neurodegenerative disorders, in the blood plasma of PD patients with varying degrees of cognitive impairment. These data showed that PDD 
patients (compared to age-matched controls) had an impaired ghrelin response. Notably, the AG:UAG ratio was reduced after fasting and postprandially, suggesting that the decrease of AG relative to UAG is a potential indicator of cognitive decline.

\subsection{Ageing and Ghrelin}

The effect of ageing on plasma ghrelin levels in C57BL/6 mice has been studied by Yuxiang Sun in the lab of Roy Smith. The TG and AG levels of 70 male and female C57BL/6 mice, ranging from 5 - 35 months of age, were analysed. Bloods were collected from the tail vein following a 24 hour fast and analysed by radioimmunoassay for each target. Plasma levels of TG increased significantly and AG was increased, but not reaching significance $(\mathrm{p}=0.059)$ with advancing age; however ghrelin activity (AG x 100/TG) was also measured and showed no correlation with age. Also, mRNA levels of ghrelin from the brains of young (2-6 months), middle-aged (12-18 month) and aged (24-28 month) mice were analysed by quantitative real-time PCR. The 2, 6, 12 and 28-month old mice had very similar expression, whereas the 18 and 24 month old mice showed a significant increase in ghrelin mRNA expression. This increase could indicate an offset response to negative energy balance caused in ageing (Sun, Garcia, \& Smith, 2007).

In addition to the work in the chapter 4 that showed AHN was unaffected in 15-17 month WT C57BL/6, the TG levels in these C57BL/6 WT and Ghrelin KO mice were analysed. There were mice of varying ages within this study (WT 6-7 months, 8-10 months and 15-17 months; Ghrelin KO 6-7 months, 8-10 months and 15-17 months of age) allowing us to quantify not only the effect of genotype on TG levels, but the effect of ageing. ELISA analysis of plasma that was collected from trunk blood (Figure 7.1), revealed no change in TG concentration with increasing age (Tukey's multiple comparison: WT 6-7 month vs WT 8-10 month, P = 0.9841; WT 6-7 month vs 
WT 15-17 month, $\mathrm{P}=0.9927)$. These data are in contrast to the findings in Sun, Garcia, \& Smith, 2007. There may be a difference between these data sets due to the age ranges used; the oldest mice used in the Yuxiang Sun study were 35 months old, whereas the oldest mice were only 17 months old in my study.

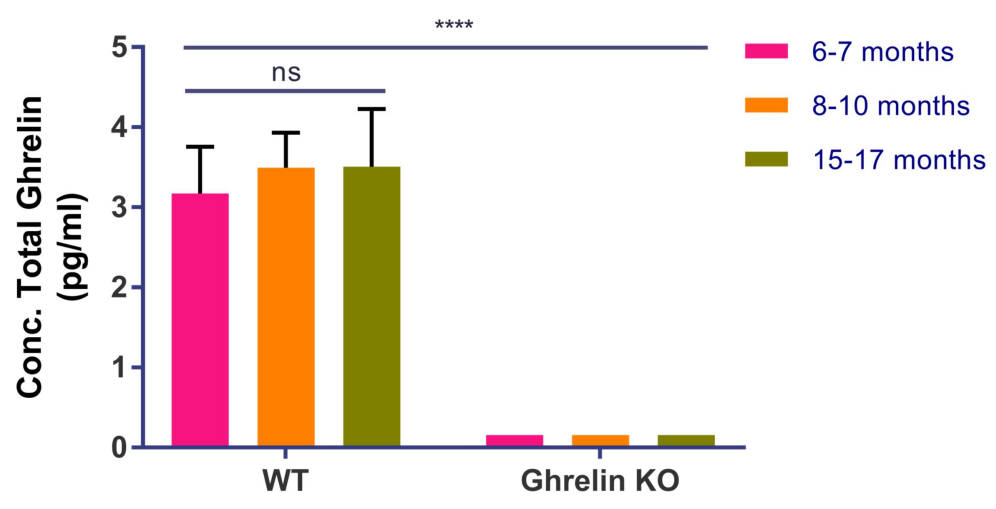

Figure 7.1 Blood plasma levels of Total Ghrelin of WT and Ghrelin KO mice at different ages.

Comparison of age against TG levels in ghrelin KO and WT mice. Age showed no effect on TG concentration in WT mice, no TG was detectable in ghrelin KO mice. $N=4 / 7$ per group. Statistical analysis performed Two-way ANOVA followed by Tukey's post-hoc multiple comparisons test. **** $=$ $\mathrm{P}<0.0001$.

The somatotropic axis (GH - IGF-1 action) is known to decline with age and these circulating factors are important for regulation of cell function and development (Ashpole, Sanders, Hodges, Yan, \& Sonntag, 2016). These factors were also examined in Sun et al. 2007. IGF-1 plasma levels were analysed in male C57BL/6 mice aged 3 - 29 months, no change was observed with increasing age. GH release was measured following 0.5 and 15 minutes after ghrelin treatment (i.p. 50mg/kg) in mice aged between 7 and 30 months; GH response to ghrelin was not affected by increasing age (Sun et al., 2007). The plasma analysis of IGF-1 and GH performed in chapter 5 also showed no changes in response to an indirect elevation of ghrelin (via CR) in healthy controls and PD patients. 


\subsection{GPCR Dimerization}

Inactive GHSR1a (apo-GHSR1a) has been to shown to dimerise with the dopamine receptor DRD1 in hippocampal neurones, which form preassembled heteromeric complexes in these neurones. Activation of this complex by DRD1 agonists enhances expression of synaptic plasticity markers in the hippocampus, by promoting NMDA-R exocytosis and synaptic reorganisation. In the absence of GHSR1a, DRD1 signalling is blocked, which also causes inhibition of DRD1-mediated hippocampal dependant memory. From the work presented in chapter 4 we know that GHSR is required for the CR-mediated increase in neurogenesis observed in WT but not GHSR KO mice. Also, the absence of circulating ghrelin had no effect on levels of new neurone production in ghrelin $\mathrm{KO}$ mice. A possible explanation for the positive effects of CR on AHN through GHSR could be related to another circulating factor that promotes AHN. As described above, GHSR1a can dimerize with DRD1 (Kern et al., 2015), and dopamine has been shown to play a role in AHN via signalling through DRD1 (Takamura et al., 2014).

GHSR1a has also been shown to dimerise with the serotonin receptor, 5HT2C. Both of these GPCR's play roles in the control of appetite and energy homeostasis. The interaction of this heteromer has been shown to inhibit calcium signalling and is expressed in primary hippocampal and hypothalamic rat neurones in culture. When $5 \mathrm{HT} 2 \mathrm{C}$ signalling is blocked the orexigenic effects of ghrelin are enhanced, however, treatment with a 5HT2C agonist attenuates these effects (Schellekens et al., 2015). Interestingly, decreasing the central levels of serotonin (5HT) enhances AHN in mice. The hTM-DTA mouse model, that results in a reduction in 5HT-ergic neurones in the brain, showed significant increases in immature DCX-positive neurones, enhanced proliferation of NSPCs (shown by increases in $\mathrm{BrdU}^{+} / \mathrm{MCM}^{+}$ (minichromosome maintenance protein 2 ) and $\mathrm{BrdU}^{+} / \mathrm{Ki}^{+} 7^{+}$cells in the $\mathrm{SGZ}$ ) and increased new adult-born neurone survival $\left(\mathrm{BrdU}^{+} / \mathrm{NeuN}^{+}\right.$cells in the 
SGZ) (N. N. Song et al., 2016). These data indicate that 5HT plays an important role in regulating $\mathrm{AHN}$ and that acyl-ghrelin mediated AHN may involve modulation of $5 \mathrm{HT} 2 \mathrm{C}$.

\subsection{Ageing and Activation of Ghrelin}

Ageing may affect the enzymatic processes that result in the acylation of ghrelin. Age may have an effect on the levels or activity of the ghrelin activating enzyme, GOAT. A study looking at the effects of a 5 month long period of CR ( $35 \%$ reduction in calories compared to ad-lib fed group) on plasma ghrelin levels and GOAT mRNA expression in young 5 week old and adult 5 month old rats reported that GOAT mRNA expression in stomach was increased 21-fold in adult ad-lib fed rats compared to young ad-lib fed rats, and was 14-fold higher in adult CR rats compared to adult ad-lib rats. There was a 2.5 fold elevation in UAG levels and TG levels in the adult CR and young ad-lib rats compared to adult ad-lib (Reimer, Maurer, Lau, \& Auer, 2010). Previous to this, another study showed that GOAT mRNA expression was unchanged in the gut mucosa of male Sprague-Dawley rats of increasing age (10, 25 and 60 days), indicating stability with age (González, Vázquez, López, \& Diéguez, 2008). These two studies may have elicited differing results due to the fact they used different strains of rat; Reimer study used male Wistar rats whereas the Gonzalez study used male Sprague-Dawley rats. However, it is more likely due to the length of their CR regimes; the Reimer study $C R^{\prime} d$ its rats for 5 months, whereas the Gonzalez study only used an acute 48-hour CR.

As we know, ghrelin is acylated by the addition of octanoic acid to serine $\left(\mathrm{Ser}^{3}\right)$, but the majority of circulating ghrelin is the inactive form, UAG. Acylprotein thioesterase 1 (APT1), also known as lysophospholipase 1 (LYPLA1), has been identified as a de-acylation enzyme for ghrelin in the stomach. Homogenate of rat stomach was separated by ammonium sulphate 
precipitation and chromatography, and further separated by SDS-PAGE and Western blotting (Shanado, Kometani, Uchiyama, Koizumi, \& Teno, 2004). Further analysis on APT1's deacylating activity was done by mass spectrometry of fetal bovine serum (FBS) and HepG2 cells (Satou et al., 2010). APT1 may have a role in memory and ageing; in a study comparing 'young' 6-month old and 'aged' 26-month old male mice, the expression of microRNA-138 (controller of APT1 translation) and APT1 in the hippocampus was examined. The mice were also subject to the hippocampal dependent novel object recognition (NOR) memory test. The aged mice had impaired memory function and in-situ hybridisation showed increased hippocampal miR-138 expression in mice that had no memory impairment. APT1 mRNA from hippocampal homogenates was quantified by qPCR, which was increased in the aged mice but not memory impaired mice. Cells that exhibited higher miR-138 expression had reduced APT1 protein expression. This work suggesting that APT1 and miR-138 play a role in agerelated memory function (Tatro et al., 2013).

\subsection{Adult Hippocampal Neurogenesis in Ageing}

AHN in humans is a highly debated topic. The seminal study performed on human brain tissue in the lab of Fred Gage in 1998 brought to light the ability of the human brain to produce new neurones throughout life. They used post-mortem human brain tissue (hippocampus and SVZ sections) from adult cancer patients ( $n=5$, aged $58-72$ ) that had been administered the thymidine analogue, $\mathrm{BrdU}(250 \mathrm{mg} ; 2.5 \mathrm{mg} / \mathrm{ml}, 100 \mathrm{ml}$ ), during their treatment. BrdU incorporated into the DNA of dividing cells and was detected immunohistochemically. Analysis of BrdU immunoreactivity revealed BrdU-positive cells in the GCL of the DG and the SVZ. To determine whether these new adult-born cells were of a neuronal lineage, triple immunohistochemical staining was performed using the mature 
neuronal marker NeuN and the astrocyte marker GFAP, in conjunction with BrdU. BrdU-NeuN positive cells were shown in the adult human DG, indicating that the human adult brain has the ability to generate new neurones throughout the course of life (Eriksson et al., 1998).

Another study supporting the idea that neurogenesis occurs in the adult brain was performed in the lab of Jonas Frisén. Hippocampal neuronal cells were isolated from post-mortem adult brains (aged 19 - 92) by flow cytometry with an anti-NeuN antibody, and birth dated using carbon-14 $\left.{ }^{14} \mathrm{C}\right) .{ }^{14} \mathrm{C}$ content in the genomic DNA of the hippocampal neurones, assessed by accelerator mass spectrometry, revealed that the cellular concentration of ${ }^{14} \mathrm{C}$ correlated with atmospheric ${ }^{14} \mathrm{C}$ after the individual was born, confirming postnatal hippocampal neurogenesis. The eldest individual studied displayed higher cellular ${ }^{14} \mathrm{C}$ levels than that found in the atmosphere before 1955, this individual was 42 in 1955, indicating that neurogenesis continued past the $5^{\text {th }}$ decade of life (Spalding et al., 2013).

However, more recently there has been data questioning the process of $\mathrm{AHN}$ in humans. Work from the lab of Arturo Alvarez-Buylla reported that neurogenesis declines drastically in children and is almost indiscernible in adults. Human post mortem brain tissue was used from 14 gestational weeks (GW) to 77 years of age, with donors suffering from a range of clinical conditions such as sepsis, heart failure, pneumonia and respiratory failure. Also, the rhesus macaque (macaca mulatta) hippocampus was examined as part of their study. Using immunohistochemical techniques they showed that proliferation of cells in the human DG (Ki67) declines rapidly with increasing age from $14 \mathrm{GW}$ to 1 year, and that proliferating progenitor cells $\left(\mathrm{Ki} 67^{+} / \mathrm{SOX} 1^{+}, \mathrm{Ki} 67^{+} / \mathrm{SOX} 2^{+}\right)$also decreased rapidly within the $1^{\text {st }}$ year and didn't form in the SGZ. Furthermore, young neurone $\left(\mathrm{DCX}^{+} / \mathrm{PSA}^{\left.-\mathrm{NCAM}^{+}\right)}\right.$ number also decreased from infancy to childhood (7/13 years old), with very few immature neurones seen after 13 years of age. In the macaque hippocampus, between birth and 1.5 years of age the number of proliferating 
$\mathrm{Ki}^{+}{ }^{+}$cells in the SGZ declined eight-fold. This decreased a further 35-fold from 1.5 years to 7 years of age, with the SGZ becoming less defined with

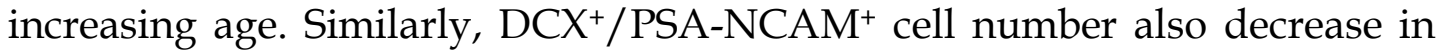
the same time-frame, becoming extremely rare by 7 years of age. Two 1.5year old and one 7-year old macaque were administered BrdU (50mg/kg, 2 times a day for 5 days) in order to subsequently identify new adult-born young neurones $\left(\mathrm{DCX}^{+} / \mathrm{BrdU}^{+}\right)$and new-born mature neurones $\left(\mathrm{NeuN}^{+} / \mathrm{BrdU}^{+}\right)$. These cell types were observed in the GCL of 1.5-year old macaques, but not in the 7-year old. These data indicate that neurogenesis declines rapidly in the $1^{\text {st }}$ year of life (Sorrells et al., 2018).

These opposing studies indicate that more work needs to be done in order to understand the mechanisms of AHN in humans. We know that AHN occurs in mice and rats, shown by the work in this thesis, and that it occurs in various other species. Age-dependent neurogenesis has been shown in birds, such as the homing pigeon. Birds that require long-distance navigation such as the homing pigeon develop their ability to navigate in the first few months they gain the ability of flight. However, they also require the ability to remember information of their environment needed for navigation in the future. Homing pigeons ranging from 1 month - 14 years of age were analysed, one hemisphere of the brain was sectioned to determine neurone number using Giemsa stain (a stain that has a high affinity for neurones), and the other hemisphere was used to analyse DCX immunoreactivity in the hippocampus. Along with increased hippocampal volume, there was a large increase in the number of neurones with increasing age, approximately 740,000 in 1-month old pigeons to 1.9 million neurones in the old pigeon of 14 years. In comparison, the number for $\mathrm{DCX}^{+}$ neurones decreased with advancing age, from approximately 20,000 in 1 month to 11,000 in 14 year old birds, with the sharpest decline happening within the $1^{\text {st }}$ year of life (Meskenaite, Krackow, \& Lipp, 2016). This is also reported in the hippocampal DG of rhesus monkeys (Macaca mulatta) ranging from 6.1 to 31.5 years of age $(n=42)$. Using immunohistochemistry they 
demonstrated a $68 \%$ decline in cell proliferation $\left(\mathrm{Ki}^{+} 7^{+}\right)$and a 53\% decline in survival capacity in new-born cells, as some monkeys were administered BrdU 3 weeks before perfusion in the aged DG. There was also an age-related decline in immature neurone $\mathrm{DCX}^{+}$cell number in the GCL of the DG. Moreover, new adult-born immature neurones $\left(\mathrm{BrdU}^{+} \mathrm{DCX}^{+}\right)$and new adultborn mature neurones $\left(\mathrm{BrdU}^{+} \mathrm{NeuN}^{+}\right)$also decline in number with advancing age (Ngwenya, Heyworth, Shwe, Moore, \& Rosene, 2015).

Of late it has been shown that there are conserved properties concerning embryonic and post-natal DG neurogenesis. Single-cell RNA sequencing has revealed 4 distinguishable groups of cells, immune cells (microglia and macrophages), vascular cells (endothelial cells), glial cells, and neuronal cells (immature and mature dentate granule cells, RGLs) in the DG's of mice ranging from embryonic day (E) 16.5 to post-natal day (P) 132. These cell types are unchanged over the course of time, but the quantity of these cells differ through the various stages of development and maturation, along with where they are found in the DG. RGL's of the DG seemed to be mostly quiescent during early development, but after re-entering the cellcycle at later post-natal stage they become neuronal intermediate progenitor cells (nIPC's) and commit to a neuronal lineage. This process was conserved from E16.5 to P132 (Hochgerner et al., 2018).

As is commonly described, the production of new neurones by AHN and their functional integration into existing circuits contributes to enhancing learning and memory. It has been shown that AG-mediated increases in AHN result in improved pattern separation memory function (the ability to discriminate between similar contexts) in rats (Kent et al., 2015). With increasing age there are age-related cognitive impairments in humans. A study comparing the effects of age on spatial pattern separation in cognitively normal young ( $18-25$ years old, $n=30$ ) and old ( $>65$ years, $n$ =30) adult humans has been performed. Participants were asked to take part in a delayed match-to-sample task that had various degrees of spatial 
separation. In the sample phase, a circle was shown on screen and the individual was asked to remember its location. In the choice phase, 2 circles were shown and the participant was asked to indicate which circle was in the same location as observed in the sample phase. The 2 circles were separated by $0,0.5,1.0$ or $1.5 \mathrm{~cm}$. Both groups showed increased memory function with increasing separation of the circles, but the young group out-performed the old group in accurately identifying the sample circle, indicating a reduction in efficiency with increasing age (Holden et al., 2012). This age-related decline in pattern separation function may be due to structural and functional changes to the DG/CA3 hippocampal network. High resolution BOLD fMRI scanning on DG/CA3 activity was performed in 20 young (mean age 21 years) and 20 old adults (mean age 71 years), whilst participating in a pattern separation task viewing pictures of every-day objects to distinguish if they were indoor or outdoor items, some of which were repeated or similar (lure activity). No difference in DG/CA3 fMRI activity was reported between young and old adults with distinct stimuli, but when stimuli were similar the DG/CA3 response was reduced in the older group (representational rigidity). Ultra-high resolution diffusion imaging showed changes to DG/CA3 dendrites that correlated with the rigidity observed in the older group (M. A. Yassa, Mattfeld, Stark, \& Stark, 2011).

Dysfunction of AHN has been shown in models of AD. The APP-PS1 model of $\mathrm{AD}$ that expresses chimeric mouse/human amyloid precursor protein and human presenilin (female 3, 10 and 13-month old), and the Tg2576 mouse model that over expresses human APP ( 3 and 5 month old), were used to determine rates of AHN in AD. The APP-PS1 mice received daily i.p. injections of $\mathrm{BrdU}(10 \mathrm{mg} / \mathrm{ml}$ at $50 \mathrm{mg} / \mathrm{kg}$ body weight) for 5 days, 4 weeks prior to being euthanized, in-order to analyse cell survival. Using immunohistochemical techniques to show hyper-proliferation of immature neurones $\left(\mathrm{PCNA}^{+} \mathrm{DCX}^{+}\right.$) in the GCL of the $\mathrm{DG}$, specifically in the pre-plaque stage in both models (APP-PS1, 3 months; Tg2576, 3 months), there was a 
significant increase compared to WT controls, which was not seen in the older ages of either model. An increase in proliferating cell number (PCNA ${ }^{+}$) was also reported in 3-month old mice of both models, but was significantly reduced in older mice. Also, in the 3-month old APP-PS1 mice, the rate of new DG cell survival (BrdU+) was reduced compared to WT controls, as was the number of new adult-born neurones $\left(\mathrm{BrdU}^{+} / \mathrm{NeuN}^{+}\right)$. This work indicates, even in the pre-plaque stages of $\mathrm{AD}$, that $\mathrm{AHN}$ is negatively affected (M. S. Unger et al., 2016).

\subsection{Calorie Restriction and Ageing}

As discussed in the introduction to this thesis, the effects of CR are conserved across various species. The work presented in chapter 3 indicates that elevating levels of endogenous ghrelin by CR promotes $\mathrm{AHN}$ and cell activation in murine models. Similar outcomes have been witnessed in other studies.

Work performed in Rozalyn Anderson's group studied the effect of CR in rhesus monkeys. A NIA (National Institute of Ageing) study to look at the effects of CR and the age of on-set of CR began in 1987. Using a mix of male and female monkeys were grouped in young-onset (juvenile, adolescent and adults) or old-onset (16-23 years old) CR. They found that males lived longer than females, independent of whether they were control animals or CR or the on-set of CR. This study found that in the old-onset CR group of monkeys' beneficial metabolic changes such as reduced weight and decreased cholesterol. The young onset group did not show any of the expected benefits of CR such as reduced serum glucose levels. However the incidence of cancer, diabetes and cardiovascular disease was not reduced, but the detection of these diseases occurred later in the CR monkeys compared to the controls (Mattison et al., 2012). This contradicts work done previously at the Wisconsin National Primate Research centre, another 20 year study on the 
effects of adult onset CR on rhesus monkeys. They showed that a 30\% CR reduced the incidence of deaths due to ageing, where $50 \%$ of the control group animals survived at a time-point compared to $80 \%$ of CR animals survived (Colman et al., 2009).

The effects of CR have also been examined in elderly humans. In a study of 50 healthy elderly subjects aged between 50 -80 years old and BMI >21, 29 were female with the average age of 60.5 and an average BMI of 28 . The CR group underwent 3 months of a 30\% reduced calorie diet, where the control group were told not to change their eating habits. Compared to the control group, the CR subjects had significant decreases in weight and BMI, along with decreased levels of fasting insulin and increased verbal memory score (Witte et al., 2009).

These studies show that CR is beneficial not only in promoting longevity and improving memory function, but can off-set the incidence of age related diseases. This is a method that could be adopted as a preventative measure against disease in ageing.

\subsection{Drug development for Ghrelin}

The ghrelin axis has been a target for drug development in multiple disorders.

One such disorder is Prader-Willi syndrome (PWS). The syndrome is characterised by increased circulating AG and reduced UAG, accompanied by excessive eating or hyperphagia (Kuppens et al., 2015; Tauber et al., 2014). In a recent clinical trial, the UAG analogue AZP-531 that functionally blocks AG activity was tested for its effects on food behaviour in PWS. This was a multi-centre (France, Spain and Italy), double-blind, randomized trial, with a placebo control. 47 clinically diagnosed patients (male and female, aged 1250 years) with PWS received placebo $(\mathrm{n}=24)$ or AZP-531 $(3-4 \mathrm{mg} / 50-70 \mathrm{~kg}, \mathrm{n}$ 
= 23) for 14 days via subcutaneous injection. Behaviour towards food was assessed with a 9-point hyperphagia questionnaire (HQ) on days 1 and 14; AZP-531 treated patients showed improvements in their 9-point HQ score (less time talking about food, less bargaining for food, less upset when denied food and getting up less at night to obtain food). Bloods were also taken from participants on day 1 and 14 following overnight fast and collected 30 minutes and 180 minutes after breakfast. There was a significant decrease in blood glucose levels in AZP-531 vs placebo treated, and was even greater in those who also had type-2 diabetes. AZP-531 treated patients also exhibited a reduction in fat mass and waist circumference from their baseline measurements. No change was seen in AG or UAG levels or ratio (Allas et al., 2018).

Another compound, CF801, a synthetic peptide that is presumed to be a GOAT inhibitor, has been shown in mice to be a potential treatment for ghrelin-based obesity. Male C57BL/6 mice were used to observe the effects of CF801 treatment (low does; $11 \mu \mathrm{mol} / \mathrm{kg}$, high dose; $22 \mu \mathrm{mol} / \mathrm{kg}$, via i.p injection once a day for 4 days) on plasma AG levels and rebound feeding after fasting. Mice given the high dose (HD) of CF801 displayed significantly reduced plasma AG levels compared to vehicle treated controls ( $50 \%$ reduction in AG), and following a 24 hour fast HD CF801 treated mice consumed significantly less chow than vehicle treated mice. HD CF801 treated mice had reduced fat mass and body weight gain, and attenuated inclination for high-fat food. Also, CF801 treated SG-1 cells, that are derived from stomach ghrelinomas, had reduced AG release (Wellman et al., 2015).

Ghrelin receptor agonist, TZP-101, has been shown to promote gastric emptying (gastroparesis), which is a symptom of PD and diabetes. Adults with type 1 or type 2 (male and female, aged 18-65) diabetes exhibiting between moderate and severe symptoms of gastroparesis, were treated with placebo $(n=10)$ or varying doses of TZP-101 $(n=14)$ via intravenous infusion, and fasted for 10 hours before receiving a standardized 
radiolabelled low-fat meal and drink. TZP-101 treatment resulted in 20\% increase in gastric emptying and a 34\% reduction in latency of gastric and liquid emptying after a single dose. Plasma and serum were also obtained at 0, 15, 45, 90, 180, 360 minutes, 12 and 24 hours after dosing, to measure GH, IGF-1 and ghrelin levels. All patients that received TZP-101 had increased GH plasma concentrations, but no effect was seen in IGF-1 and octanolyated ghrelin levels. The study concluded that the ghrelin receptor agonist improved gastric emptying in diabetes (Ejskjaer et al., 2009).

Drugs can also be repurposed for different diseases and disorders. An example of this is the GlaxoSmithKline drug Rosiglitazone, which was initially designed to treat insulin-resistance in diabetes, has been shown to improve cognitive deficits in AD models. In a double-blind parallel-group pilot study, 30 participants with early AD and amnestic MCI (aged 55-85) were randomised and treated for 6 months with either placebo $(n=10)$ or $4 \mathrm{mg}$ daily dose of Rosiglitazone $(\mathrm{n}=20)$. Cognitive testing was performed, assessing verbal memory, selective attention and category fluency. Following 4 and 6 months of rosiglitazone treatment, participants had improvements in delayed recall and selective attention compared to the placebo group. Plasma amyloid- $\beta$ levels were also measured, the placebo group showed declining levels of amyloid- $\beta$ which is observed in AD progression, whereas the rosiglitazone treated subjects had unchanged levels after 6 months. These findings indicate that rosiglitazone as a potential treatment for cognitive decline in AD (Watson et al., 2005). Subsequently, in a mouse model of AD that overexpresses human amyloid precursor protein (hAPP), were chronically treated for 4 weeks with rosiglitazone $(5 \mathrm{mg} / \mathrm{kg} /$ day $)$. After treatment the mice were assessed using the object recognition task, and after 16 weeks were assessed using the Morris water maze test (spatial memory task); both analyses showed cognitive improvements compared to control mice. Notably, after 16 weeks of treatment these mice showed reduced levels of amyloid- $\beta$ in the brain and a reduction in amyloid- $\beta$ plaque deposition in 
the hippocampus. These pre-clinical findings provide further support for rosiglitazone treatment in AD (Escribano et al., 2010).

GHSR agonists have shown to elicit improvements in cognition in mouse models. One study using the non-peptide GHSR agonists GSK894490A and CP-464709-18 in male Lister hooded rats, who after administration of the compounds ( 0.3 and $3 \mathrm{mg} / \mathrm{kg}$, orally). 2 hours following dosing the rats were then assessed in a novel object recognition test, following habituation of the test cage and Trial 1 where objects were placed in the cage for the rat to explore and 24 hours later Trial 2 where one of the objects was replaced with a novel object, and the exploring times were recorded. Both treatments resulted in increased exploration time of the novel object compared to the familiar object. These rats were also assessed in the Atlantis water maze task, where they are required to locate a hidden platform using spatial cues, after a training session with the platform raised. Both agonist treatments (administered 1-2 hours before the task) decreased the latency time to locate the platform and path length they took. These results indicate that these agonists can cross the BBB and elicit positive effects on cognition (Atcha et al., 2009). Another GHSR agonist that has shown to improve cognition is LY444711. Male 3-month old Sprague-Dawley rats had their behaviour assessed in the spontaneous alternation plus-maze task following 1 subcutaneous injection of LY444711 $(0.25 \mathrm{ml} 5 \mathrm{mg} / \mathrm{kg}) 30$ minutes prior to being placed in the centre of the maze, the number of entries to each arm were recorded. Compared to saline treated controls, the agonist treated rats demonstrated significant improvements in alternation performance, indicating improved spatial memory performance (Diano et al., 2006).

These findings show that GHSR as a potential drug target for the treatment of disorders where cognition is impaired. 
In conclusion, this thesis presents evidence demonstrating that the ghrelin-axis is an important regulator of AHN in rodents. However, more work is needed to unearth the underlying mechanism(s) of action. Furthermore, with regard to neurodegenerative disorders linked with dementia in humans, we provide evidence that AG:UAG may be an useful diagnostic biomarker. These data suggest that subsequent studies should focus on the therapeutic potential of increasing the bio-availability of acylghrelin to treat neurodegenerative disorders associated with cognitive impairment. 


\section{Chapter 8 Appendix}

\subsection{Buffer and Reagent Recipes}

\subsubsection{0x Phosphate Buffered Solution (PBS) (0.1M), pH 7.4}

To make a 1L bottle of 10x PBS:

$>10.9 \mathrm{~g}$ Sodium phosphate disbasic $\left(\mathrm{Na}_{2} \mathrm{HPO}_{4}\right)$ (Sigma-Aldrich, S3264$1 \mathrm{KG})$

$>3.2 \mathrm{~g}$ Sodium dihydrogen orthophosphate dehydrate $\left(\mathrm{NaH}_{2} \mathrm{PO}_{4}\right)$ (Fisher Scientific, S/3720/60)

$>$ 90g Sodium Chloride (Fisher Scientific, S/3120/60)

$>1 \mathrm{~L}$ distilled $\mathrm{H}_{2} \mathrm{O}$

\subsubsection{1x Phosphate Buffered Solution (PBS) (0.01M)}

To make a 1L bottle of $1 x$ PBS:

$>100 \mathrm{ml}$ of $10 \mathrm{x}$ PBS

$>900 \mathrm{ml}$ distilled $\mathrm{H}_{2} \mathrm{O}$

\subsubsection{0x Tris Buffered Solution (TBS) pH 7.6}

To make make $1 \mathrm{~L}$ bottle of 10x TBS:

24.22g Tris [Tris(hydroxymethyl)aminomethane] (Melford, B2005)

80g Sodium Chloride (Fisher Scientific, S/3120/60)

$>1 \mathrm{~L}$ deionised $\mathrm{H}_{2} \mathrm{O}$

\subsubsection{X Tris Buffered Solution (TBS)}

To make $200 \mathrm{ml}$ of $1 x$ TBS:

20ml 10x TBS 
$>180 \mathrm{ml}$ distilled $\mathrm{H}_{2} \mathrm{O}$

\subsubsection{1\% PBS-T}

To make $200 \mathrm{ml}$ :

$>200 \mathrm{ml} 1 \mathrm{x}$ PBS

$200 \mu 1$ Triton X-100 (Sigma-Aldrich, T8787)

\subsection{3\% PBS-T}

To make 200ml:

$200 \mathrm{ml} 1 \mathrm{x}$ PBS

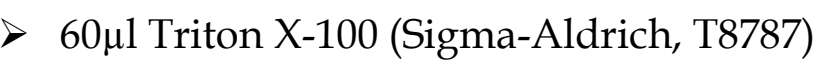

\subsubsection{M Hydrochloric Acid (HCl)}

To make $100 \mathrm{ml}$ :

16.4ml Hydrochloric Acid ( 37\%) (Fisher Scientific, H/1200/PB08

$>$ 83.6ml distilled $\mathrm{H}_{2} \mathrm{O}$

\subsubsection{Citrate Buffer pH6}

To make 1L:

2.94g Sodium citrate dehydrate (Fisher Scientific, BP327-1)

$>1 \mathrm{~L}$ distilled $\mathrm{H}_{2} \mathrm{O}$

$>$ Adjust $\mathrm{pH}$ to $\mathrm{pH} 6$ with $2 \mathrm{M} \mathrm{HCl}$

> 0.5ml Tween20 (Sigma-Aldrich, P9416)

\subsection{Sodium Acetate pH 6}

To make 500ml:

4.1g Sodium Acetate anhydrous (Sigma-Aldrich, S-2889)

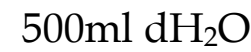


$>$ Adjust to $\mathrm{pH} 6$ with Acetic Acid

\subsection{Sodium Acetate}

To make 1L:

$>$ 16.406g Sodium Acetate anhydrous (Sigma-Aldrich, S-2889)

$>1 \mathrm{~L} \mathrm{dH_{2 } \mathrm { O }}$

\subsubsection{1\% Sodium Azide}

To make $10 \mathrm{ml}$ :

0.1g Sodium Azide (Sigma-Aldrich, S2002-25G)

$>10 \mathrm{ml} \mathrm{dH_{2 } \mathrm { O }}$

\subsubsection{PBS-Azide (0.01\%)}

To make $200 \mathrm{ml}$ :

$2 \mathrm{ml}$ of $1 \%$ sodium azide

$>200 \mathrm{ml} 1 \mathrm{x}$ PBS $(0.01 \mathrm{M})$

\subsubsection{3 $30 \%$ Sucrose solution}

To make $200 \mathrm{ml}$ :

$>60 \mathrm{~g}$ Sucrose (Sigma-Aldrich, S8501-5KG)

$>200 \mathrm{ml} 1 \times \operatorname{PBS}(0.01 \mathrm{M})$

$>$ Mix and heat

\subsubsection{M Sodium Hydroxide $(\mathrm{NaOH})$ solution}

To make $100 \mathrm{ml}$ :

20g Sodium Hydroxide pellets (Fisher Scientific, BP359-500)

$>$ Mix into $80 \mathrm{ml}$ of $\mathrm{dH}_{2} \mathrm{O}$ (exothermic reaction)

$>$ Make up to $100 \mathrm{ml}$ with $20 \mathrm{ml} \mathrm{dH}_{2} \mathrm{O}$ 


\subsubsection{4\% Paraformaldehyde (PFA) solution}

This must be performed in a fume cupboard

$100 \mathrm{ml} 1 \mathrm{x}$ PBS in a measuring cylinder

> In a separate PFA labelled jar add 4g PFA (Sigma-Aldrich, P6148-1KG)

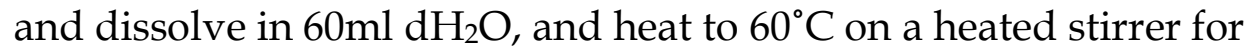
approximately 5-10 minutes.

$>$ Once the solution has become milky add a few drops of $5 \mathrm{M} \mathrm{NaOH}$ until the solution becomes clear.

$>$ Filter the solution with filter paper into a clean conical flask, and mix in the $1 \times$ PBS.

\subsubsection{Glucose Oxidase}

To make $8 \mathrm{ml}$ :

$>8 \mathrm{ml} \mathrm{dH}_{2} \mathrm{O}$

$>0.04 \mathrm{~g}$ Glucose Oxidase from Aspergillus niger (Sigma-Aldrich, G7141$50 \mathrm{KU})$

Aliquot out into $500 \mu \mathrm{l}$ aliquots

\subsubsection{Nickel enhanced Diaminobenzidine (Ni-DAB)}

To make 200ml:

In one beaker place $100 \mathrm{ml} 0.2 \mathrm{M}$ Sodium Acetate:

- $5 g$ Nickel Sulphate Hexahydrate (Sigma-Aldrich, N4882-250G)

○ 0.4g D-Glucose anhydrous (Fisher Scientific, G/0500/53)

○ $0.08 \mathrm{~g}$ Ammonium Chloride (Fisher Scientific, A/3880/53)

In $100 \mathrm{ml} \mathrm{dH}_{2} \mathrm{O}$ :

○ 0.05g 3,3-Diaminobezidine (DAB) (Sigma-Aldrich, D-8001)

- Gently heat to dissolve and stir for approximately 10 minutes.

$>$ Mix the 2 solutions together, then filter with a syringe and filter disc into foil wrapped $15 \mathrm{ml}$ tubes. 
Store at $-20^{\circ} \mathrm{C}$.

\subsubsection{Biotinylated Tyramide (bTyr)}

To make 50ml:

$>50 \mathrm{ml}$ Deionised water $\left(\mathrm{ddH}_{2} \mathrm{O}\right)$

$>0.433 \mathrm{~g}$ Boric Acid $\left(\mathrm{H}_{3} \mathrm{BO}_{3}\right)$ (Sigma-Aldrich, B7660)

$>0.286$ g Sodium Borate, Borax $\left(\mathrm{NA}_{2} \mathrm{~B}_{4} \mathrm{O}_{7} .10 \mathrm{H}_{2} \mathrm{O}\right)$ (Sigma-Aldrich, S964025G)

$>$ Check that the $\mathrm{pH}$ is at $\mathrm{pH} 8.0$, adjust where appropriate.

$>0.125 \mathrm{~g}$ Biotinamidoheaxanoic acid N-hydroxysiccinimide ester (NHSLC-biotin) (Sigma-Aldrich, B2643-250G)

$>0.039 \mathrm{~g}$ Tyramide Hydrochloride (Sigma-Aldrich, T2879-1G)

$>$ Cover the mixture and stir for 16 hours at RT.

$>$ Filter the solution through a $0.2 \mu \mathrm{m}$ filter, then aliquot and store at $20^{\circ} \mathrm{C}$. 


\section{Bibliography}

Aiello, M., Eleopra, R., \& Rumiati, R. I. (2015). Body weight and food intake in Parkinson's disease. A review of the association to non-motor symptoms. Appetite, 84, 204-211.

http://doi.org/10.1016/j.appet.2014.10.011

Aimone, J. B., Li, Y., Lee, S. W., Clemenson, G. D., Deng, W., \& Gage, F. H. (2014). Regulation and Function of Adult Neurogenesis: From Genes to Cognition. Physiological Reviews, 94(4), 991-1026. http://doi.org/10.1152/physrev.00004.2014

Alam, M. J., Kitamura, T., Saitoh, Y., Ohkawa, N., Kondo, T., \& Inokuchi, K. (2018). Adult Neurogenesis Conserves Hippocampal Memory Capacity. The Journal of Neuroscience, 38(31), 6854-6863. http://doi.org/10.1523/jneurosci.2976-17.2018

Albarran-Zeckler, R. G., Brantley, A. F., \& Smith, R. G. (2012). Growth hormone secretagogue receptor (GHS-R1a) knockout mice exhibit improved spatial memory and deficits in contextual memory. Behavioural Brain Research, 232(1), 13-9. http:/ / doi.org/10.1016/j.bbr.2012.03.012

Allas, S., Caixàs, A., Poitou, C., Coupaye, M., Thuilleaux, D., Lorenzini, F., ... Tauber, M. (2018). AZP-531, an unacylated ghrelin analog, improves food-related behavior in patients with Prader-Willi syndrome: A randomized placebo-controlled trial. PLoS ONE, 13(1), 1-19. http://doi.org/10.1371/journal.pone.0190849

Altman, J., \& Das, G. D. (1965). Autoradiographic and histologicalevidence of postnatal hippocampal neurogenesis in rats. The Journal of Comparative Neurology, 124(3), 319-335.

Anacker, C., Zunszain, P. A., Cattaneo, A., Carvalho, L. A., Garabedian, M. J., 
Thuret, S., ... Pariante, C. M. (2011). Antidepressants increase human hippocampal neurogenesis by activating the glucocorticoid receptor. Molecular Psychiatry, 16(7), 738-750. http:// doi.org/10.1038/mp.2011.26

Andrews, Z. B. (2011). The extra-hypothalamic actions of ghrelin on neuronal function. Trends in Neurosciences, 34(1), 31-40.

http://doi.org/10.1016/j.tins.2010.10.001

Andrews, Z. B., Erion, D., Beiler, R., Liu, Z.-W., Abizaid, a., Zigman, J., ... Horvath, T. L. (2009). Ghrelin Promotes and Protects Nigrostriatal Dopamine Function via a UCP2-Dependent Mitochondrial Mechanism. Journal of Neuroscience, 29(45), 14057-14065. http://doi.org/10.1523/JNEUROSCI.3890-09.2009

Ariyasu, H., Takaya, K., Iwakura, H., Hosoda, H., Akamizu, T., Arai, Y., ... Nakao, K. (2005). Transgenic mice overexpressing des-acyl ghrelin show small phenotype. Endocrinology, 146(1), 355-364.

http://doi.org/10.1210/en.2004-0629

Asakawa, A., Inui, A., Fujimiya, M., Sakamaki, R., Shinfuku, N., Ueta, Y., ... Kasuga, M. (2005). Stomach regulates energy balance via acylated ghrelin and desacyl ghrelin. Gut, 54(1), 18-24. http://doi.org/10.1136/gut.2004.038737

Ashpole, N. M., Sanders, J. E., Hodges, E. L., Yan, H., \& Sonntag, W. E. (2016). Growth hormone, insulin-like growth factor-1 and the aging brain. Experimental Gerontology, 34(5), 352-359. http://doi.org/10.1177/0963721414541462.Self-Control

Atcha, Z., Chen, W.-S., Ong, A. B., Wong, F.-K., Neo, A., Browne, E. R., ... Pemberton, D. J. (2009). Cognitive enhancing effects of ghrelin receptor agonists. Psychopharmacology, 206(3), 415-27. http:// doi.org/10.1007/s00213-009-1620-6

Avau, B., De Smet, B., Thijs, T., Geuzens, a, Tack, J., Vanden Berghe, P., \& 
Depoortere, I. (2013). Ghrelin is involved in the paracrine communication between neurons and glial cells. Neurogastroenterology and Motility $\square$ : The Official Journal of the European Gastrointestinal Motility Society, 25(9), e599-608. http:/ / doi.org/10.1111/nmo.12171

Aviles-Olmos, I., Dickson, J., Kefalopoulou, Z., Djamshidian, A., Kahan, J., Ell, P., ... Foltynie, T. (2014). Motor and Cognitive Advantages Persist 12 Months after Exenatide Exposure in Parkinson's Disease. Journal of Parkinson's Disease, 4(3), 337-344. http:/ / doi.org/10.3233/JPD-140364

Banks, W. A., Op, M. T., Robinson, S. M., \& Heiman, M. L. (2002). Extent and Direction of Ghrelin Transport Across the Blood- Brain Barrier Is Determined by Its Unique Primary Structure, 302(2), 822-827. http:/ / doi.org/10.1124/jpet.102.034827.Other

Bayliss, J. A., \& Andrews, Z. B. (2013). Ghrelin is neuroprotective in Parkinson's disease: molecular mechanisms of metabolic neuroprotection. Therapeutic Advances in Endocrinology and Metabolism, 4(1), 25-36. http:/ / doi.org/10.1177/2042018813479645

Bayliss, J. A., Lemus, M. B., Stark, R., Santos, V. V, Thompson, A., Rees, D. J., ... Andrews, X. Z. B. (2016). Ghrelin-AMPK Signaling Mediates the Neuroprotective Effects of Calorie Restriction in Parkinson ' s Disease, 36(10), 3049-3063. http:/ / doi.org/10.1523/JNEUROSCI.4373-15.2016

Bayliss, J. A., Lemus, M., Santos, V. V., Deo, M., Elsworth, J. D., \& Andrews, Z. B. (2016). Acylated but not des-acyl ghrelin is neuroprotective in an MPTP mouse model of Parkinson's disease. Journal of Neurochemistry, 137(3), 460-471. http:// doi.org/10.1111/jnc.13576

Beck, B., \& Pourié, G. (2013). Ghrelin, neuropeptide Y, and other feedingregulatory peptides active in the hippocampus: role in learning and memory. Nutrition Reviews, 71(8), 541-61. http:/ / doi.org/10.1111/nure.12045 
Bekinschtein, P., Kent, B. a., Oomen, C. a., Clemenson, G. D., Gage, F. H., Saksida, L. M., \& Bussey, T. J. (2014). Brain-derived neurotrophic factor interacts with adult-born immature cells in the dentate gyrus during consolidation of overlapping memories. Hippocampus, 24(8), 905-911. http:/ / doi.org/10.1002/hipo.22304

Bekinschtein, P., Kent, B. A., Oomen, C. A., Clemenson, G. D., Gage, F. H., Saksida, L. M., \& Bussey, T. J. (2013). BDNF in the dentate gyrus is required for consolidation of "pattern-separated" memories. Cell Reports, 5(3), 759-768. http:/ / doi.org/10.1016/j.celrep.2013.09.027

Berrout, L., \& Isokawa, M. (2012). Ghrelin promotes reorganization of denritic spines in cultured rat hippocampal slices. Neuroscience Letters, 516(2), 280-284. http:// doi.org/10.3174/ajnr.A1256.Functional

Bozon, B., Davis, S., \& Laroche, S. (2002). Regulated transcription of the immediate-early gene Zif268: mechanisms and gene dosage-dependent function in synaptic plasticity and memory formation. Hippocampus, 12(5), 570-7. http:/ / doi.org/10.1002/hipo.10100

Broglio, F., Gottero, C., Prodam, F., Gauna, C., Muccioli, G., Papotti, M., ... Ghigo, E. (2004). Non-acylated ghrelin counteracts the metabolic but not the neuroendocrine response to acylated ghrelin in humans. Journal of Clinical Endocrinology and Metabolism, 89(6), 3062-3065. http://doi.org/10.1210/jc.2003-031964

Camiña, J. P. (2006). Cell biology of the ghrelin receptor. Journal of Neuroendocrinology, 18(1), 65-76. http:/ / doi.org/10.1111/j.13652826.2005.01379.x

Carlini, V. P., Ghersi, M., Schiöth, H. B., \& de Barioglio, S. R. (2010). Ghrelin and memory: differential effects on acquisition and retrieval. Peptides, 31(6), 1190-3. http:/ / doi.org/10.1016/j.peptides.2010.02.021

Carlini, V. P., Schiöth, H. B., \& deBarioglio, S. R. (2007). Obestatin improves 
memory performance and causes anxiolytic effects in rats. Biochemical and Biophysical Research Communications, 352(4), 907-912.

http://doi.org/10.1016/j.bbrc.2006.11.112

Casanueva, F. F., Camiña, J. P., Carreira, M. C., Pazos, Y., Varga, J. L., \& Schally, A. V. (2008). Growth hormone-releasing hormone as an agonist of the ghrelin receptor GHS-R1a. Proceedings of the National Academy of Sciences of the United States of America, 105(51), 20452-20457. http://doi.org/10.1073/pnas.0811680106

Chahine, L. M., Stern, M. B., \& Chen-Plotkin, A. (2014). Blood-based biomarkers for Parkinson's disease. Parkinsonism and Related Disorders, 20(SUPPL.1), S99-S103. http:/ / doi.org/10.1016/S1353-8020(13)70025-7

Chandra, R., Hiniker, A., Kuo, Y.-M., Nussbaum, R. L., \& Liddle, R. A. (2017). a-Synuclein in gut endocrine cells and its implications for Parkinson's disease. JCI Insight, 2(12), 1-13. http:// doi.org/10.1172/jci.insight.92295

Chen, H. Y., Trumbauer, M. E., Chen, A. S., Weingarth, D. T., Adams, J. R., Frazier, E. G., ... Qian, S. (2004). Orexigenic action of peripheral ghrelin is mediated by neuropeptide $Y$ and agouti-related protein. Endocrinology, 145(6), 2607-2612. http:/ / doi.org/10.1210/en.2003-1596

Chen, L., Xing, T., Wang, M., Miao, Y., Tang, M., Chen, J., ... Ruan, D. Y. (2011). Local infusion of ghrelin enhanced hippocampal synaptic plasticity and spatial memory through activation of phosphoinositide 3kinase in the dentate gyrus of adult rats. European Journal of Neuroscience, 33(2), 266-275. http://doi.org/10.1111/j.1460-9568.2010.07491.x

Clelland, C. D., Choi, M., Romberg, C., Clemenson, G. D., Fragniere, a, Tyers, P., ... Bussey, T. J. (2009). A functional role for adult hippocampal neurogenesis in spatial pattern separation. Science (New York, N.Y.), 325(5937), 210-3. http:// doi.org/10.1126/ science.1173215

Colman, R. J., Anderson, R. M., Johnson, S. C., Kastman, E. K., Kosmatka, K. 
J., Beasley, T. M., ... Weindruch, R. (2009). Caloric restriction delays disease onset and mortalilty in rhesus monkeys. Science, 325(5937), 201204. http:// doi.org/10.1126/science.1173635.Caloric

Colman, R. J., Beasley, T. M., Kemnitz, J. W., Johnson, S. C., Weindruch, R., \& Anderson, R. M. (2014). Caloric restriction reduces age-related and allcause mortality in rhesus monkeys. Nature Communications, 5, 3557. http://doi.org/10.1038/ncomms4557

Cowley, M. A., Smith, R. G., Diano, S., Tscho, M., Pronchuk, N., Grove, K. L., ... Tgh, A. (2003). The Distribution and Mechanism of Action of Ghrelin in the CNS Demonstrates a Novel Hypothalamic Circuit Regulating Energy Homeostasis, 37, 649-661.

Davis, A. A., \& Racette, B. (2016). Parkinson disease and cognitive impairment: Five new things. Neurology: Clinical Practice, 6(5), 452-458. http://doi.org/10.1212/CPJ.0000000000000285

Deary, I. J., Corley, J., Gow, A. J., Harris, S. E., Houlihan, L. M., Marioni, R. E., ... Starr, J. M. (2009). Age-associated cognitive decline. British Medical Bulletin, 92(1), 135-152. http:// doi.org/10.1093/bmb/ldp033

del Rio, J. A., \& Soriano, E. (1989). Immunocytochemical detection of 5'bromodeoxyuridine incorporation in the central nervous system of the mouse. Developmental Brain Research, 49(2), 311-317. http://doi.org/10.1016/0165-3806(89)90033-3

Deng, W., Aimone, J. B., \& Gage, F. H. (2010). New neurons and new memories: how does adult hippocampal neurogenesis affect learning and memory? Nature Reviews. Neuroscience, 11(5), 339-50. http://doi.org/10.1038/nrn2822

Déry, N., Pilgrim, M., Gibala, M., Gillen, J., Wojtowicz, J. M., MacQueen, G., \& Becker, S. (2013). Adult hippocampal neurogenesis reduces memory interference in humans: opposing effects of aerobic exercise and 
depression. Frontiers in Neuroscience, 7(April), 1-15.

http://doi.org/10.3389/fnins.2013.00066

Dhurandhar, E. J., Allison, D. B., van Groen, T., \& Kadish, I. (2013). Hunger in the absence of caloric restriction improves cognition and attenuates Alzheimer's disease pathology in a mouse model. PloS One, 8(4), e60437. http://doi.org/10.1371/journal.pone.0060437

Diano, S., Farr, S. a, Benoit, S. C., McNay, E. C., da Silva, I., Horvath, B., ... Horvath, T. L. (2006). Ghrelin controls hippocampal spine synapse density and memory performance. Nature Neuroscience, 9(3), 381-8. http://doi.org/10.1038/nn1656

Dickerson, B. C., \& Eichenbaum, H. (2010). The episodic memory system: neurocircuitry and disorders. Neuropsychopharmacology : Official Publication of the American College of Neuropsychopharmacology, 35(1), 86104. http://doi.org/10.1038/npp.2009.126

Doherty, G. H., Oldreive, C., \& Harvey, J. (2008). Neuroprotective actions of leptin on central and peripheral neurons in vitro. Neuroscience, 154(4), 1297-1307. http://doi.org/10.1016/j.neuroscience.2008.04.052

Duan, W., Lee, J., Guo, Z., \& Mattson, M. P. (2001). Dietary restriction stimulates BDNF production in the brain and thereby protects neurons against excitotoxic injury. Journal of Molecular Neuroscience : MN, 16(1), 112. http://doi.org/10.1385/JMN:16:1:1

Duan, W., \& Mattson, M. P. (1999). Dietary restriction and 2-deoxyglucose administration improve behavioral outcome and reduce degeneration of dopaminergic neurons in models of Parkinson's disease. Journal of Neuroscience Research, 57(2), 195-206. http:/ / doi.org/10.1002/(SICI)10974547(19990715)57:2<195::AID-JNR5>3.0.CO;2-P

Duerrschmid, C., He, Y., Wang, C., Li, C., Bournat, J. C., Romere, C., ... Chopra, A. R. (2017). Asprosin is a centrally acting orexigenic hormone. 
Nature Medicine, 23(12), 1444-1453. http:/ / doi.org/10.1038/nm.4432

Ejskjaer, N., Vestergaard, E. T., HellstrÖm, P. M., Gormsen, L. C., Madsbad, S., Madsen, J. L., ... Kosutic, G. (2009). Ghrelin receptor agonist (TZP101) accelerates gastric emptying in adults with diabetes and symptomatic gastroparesis. Alimentary Pharmacology and Therapeutics, 29(11), 1179-1187. http:/ / doi.org/10.1111/j.1365-2036.2009.03986.x

Eriksson, P. S., Perfilieva, E., Bjork-Eriksson, T., Alborn, A. M., Nordborg, C., Peterson, D. A., \& Gage, F. H. (1998). Neurogenesis in the adult human hippocampus. Nat Med, 4(11), 1313-1317. http:/ / doi.org/10.1038/3305

Ernst, A., Alkass, K., Bernard, S., Salehpour, M., Perl, S., Tisdale, J., ... Frisén, J. (2014). Neurogenesis in the striatum of the adult human brain. Cell, 156(5), 1072-83. http:/ / doi.org/10.1016/j.cell.2014.01.044

Escribano, L., Simón, A. M., Gimeno, E., Cuadrado-Tejedor, M., De Maturana, R. L., García-Osta, A., ... Frechilla, D. (2010). Rosiglitazone rescues memory impairment in Alzheimer's transgenic mice: Mechanisms involving a reduced amyloid and tau pathology. Neuropsychopharmacology, 35(7), 1593-1604. http://doi.org/10.1038/npp.2010.32

Fabel, K., Wolf, S. A., Ehninger, D., Babu, H., Leal-Galicia, P., \& Kempermann, G. (2009). Additive effects of physical exercise and environmental enrichment on adult hippocampal neurogenesis in mice. Frontiers in Neuroscience, 3(NOV), 1-7. http:// doi.org/10.3389/neuro.22.002.2009

Farooqi, I. S., Keogh, J. M., Kamath, S., Jones, S., Gibson, W. T., Trussell, R., ... O'rahilly, S. (2001). Metabolism: Partial leptin deficiency and human adiposity. Nature, 414(6859), 34-35. http:/ / doi.org/10.1038/35102112

Fiszer, U., Michałowska, M., Baranowska, B., Wolińska-Witort, E., Jeske, W., Jethon, M., ... Marcinowska-Suchowierska, E. (2010). Leptin and ghrelin 
concentrations and weight loss in Parkinson's disease. Acta Neurologica Scandinavica, 121(4), 230-236. http://doi.org/10.1111/j.16000404.2009.01185.x

Fontana, L., Partridge, L., \& Longo, V. D. (2010). Extending healthy life span-from yeast to humans. Science (New York, N.Y.), 328(5976), 321-6. http://doi.org/10.1126/science.1172539

Gargantini, E., Lazzari, L., Settanni, F., Taliano, M., Trovato, L., Gesmundo, I., ... Granata, R. (2016). Obestatin promotes proliferation and survival of adult hippocampal progenitors and reduces amyloid- $\beta$-induced toxicity. Molecular and Cellular Endocrinology, 422, 18-30. http://doi.org/10.1016/j.mce.2015.11.008

Gauna, C., Delhanty, P. J. D., Hofland, L. J., Janssen, J. A. M. J. L., Broglio, F., Ross, R. J. M., .. Van Der Lely, A. J. (2005). Ghrelin stimulates, whereas des-octanoyl ghrelin inhibits, glucose output by primary hepatocytes. Journal of Clinical Endocrinology and Metabolism, 90(2), 1055-1060. http://doi.org/10.1210/jc.2004-1069

Ge, X., Yang, H., Bednarek, M. A., Galon-Tilleman, H., Chen, P., Chen, M., ... Kaplan, D. D. (2017). LEAP2 Is an Endogenous Antagonist of the Ghrelin Receptor. Cell Metabolism, 1-9. http://doi.org/10.1016/j.cmet.2017.10.016

González, C. R., Vázquez, M. J., López, M., \& Diéguez, C. (2008). Influence of chronic undernutrition and leptin on GOAT mRNA levels in rat stomach mucosa. Journal of Molecular Endocrinology, 41(5-6), 415-421. http://doi.org/10.1677/JME-08-0102

Gonzallez-Perez, O. (2012). Neural stem cells in the adult human brain. Biol Biomed Rep, 2(1), 59-69.

Gräff, J., Kahn, M., Samiei, A., Gao, J., Ota, K. T., Rei, D., \& Tsai, L.-H. (2013). A dietary regimen of caloric restriction or pharmacological activation of 
SIRT1 to delay the onset of neurodegeneration. The Journal of

Neuroscience: The Official Journal of the Society for Neuroscience, 33(21), 8951-60. http:/ / doi.org/10.1523/JNEUROSCI.5657-12.2013

Guan, X., Yu, H., Palyha, O. C., Kulju, K., Feighner, S. D., Sirinathsinghji, D. J. S., ... Howard, A. D. (1997). Distribution of mRNA encoding the growth hormone secretagogue receptor in brain and peripheral tissues, 23-29.

Gutierrez, J. a, Solenberg, P. J., Perkins, D. R., Willency, J. a, Knierman, M. D., Jin, Z., ... Hale, J. E. (2008). Ghrelin octanoylation mediated by an orphan lipid transferase. Proceedings of the National Academy of Sciences of the United States of America, 105(17), 6320-5. http://doi.org/10.1073/pnas.0800708105

Halagappa, V. K. M., Guo, Z., Pearson, M., Matsuoka, Y., Cutler, R. G., Laferla, F. M., \& Mattson, M. P. (2007). Intermittent fasting and caloric restriction ameliorate age-related behavioral deficits in the tripletransgenic mouse model of Alzheimer's disease. Neurobiology of Disease, 26(1), 212-20. http:/ / doi.org/10.1016/j.nbd.2006.12.019

Hanson, N. D., Owens, M. J., \& Nemeroff, C. B. (2011). Depression, antidepressants, and neurogenesis: A critical reappraisal.

Neuropsychopharmacology, 36(13), 2589-2602.

http://doi.org/10.1038/npp.2011.220

Harada, C. N., Love, M. C. N., \& Triebel, K. (2013). Normal Cognitive Aging. Clin Geriatr Med., 29(4), 737-752.

http:/ / doi.org/10.1016/j.cger.2013.07.002.Normal

Harris, K. M., Jensen, F. E., \& Tsao, B. (1992). Three-dimensional structure of dendritic spines and synapses in rat hippocampus (CA1) at postnatal day 15 and adult ages: implications for the maturation of synaptic physiology and long-term potentiation. The Journal of Neuroscience: The Official Journal of the Society for Neuroscience, 12(7), 2685-2705. 
http://doi.org/10.1016/j.tcb.2009.06.001

Hely, M. A., Reid, W. G. J., Adena, M. A., Halliday, G. M., \& Morris, J. G. L. (2008). The Sydney Multicenter Study of Parkinson's disease: The inevitability of dementia at 20 years. Movement Disorders, 23(6), 837-844. http:/ / doi.org/10.1002/mds.21956

Hering, H., \& Sheng, M. (2001). Dentritic spines: structure, dynamics and regulation. Nature Reviews Neuroscience, 2(12), 880-888. http://doi.org/10.1038/35104061

Hewson, a. K., \& Dickson, S. L. (2001). Systemic Administration of Ghrelin Induces Fos and Egr-1 Proteins in the Hypothalamic Arcuate Nucleus of Fasted and Fed Rats. Journal of Neuroendocrinology, 12(11), 1047-1049. http:/ / doi.org/10.1046/j.1365-2826.2000.00584.x

Hochgerner, H., Zeisel, A., Lönnerberg, P., \& Linnarsson, S. (2018). Conserved properties of dentate gyrus neurogenesis across postnatal development revealed by single-cell RNA sequencing. Nature Neuroscience. http:/ / doi.org/10.1038/s41593-017-0056-2

Hofmann, K. (2000). A superfamily of membrane-bound O-acyltransferases with the implications for Wnt signaling. Trends in Biochemical Sciences, 25(3), 111-112. http://doi.org/https://doi.org/10.1016/S09680004(99)01539-X

Höglinger, G. U., Rizk, P., Muriel, M. P., Duyckaerts, C., Oertel, W. H., Caille, I., \& Hirsch, E. C. (2004). Dopamine depletion impairs precursor cell proliferation in Parkinson disease. Nature Neuroscience, 7(7), 726-35. http:/ / doi.org/10.1038/nn1265

Holden, H. M., Hoebel, C., Loftis, K., \& Gilbert, P. E. (2012). Spatial pattern separation in cognitively normal young and older adults. Hippocampus, 22(9), 1826-1832. http:/ / doi.org/10.1002/ hipo.22017 
Hopkins, A. L., Nelson, T. A. S., Guschina, I. A., Parsons, L. C., Lewis, C. L., Brown, R. C., ... Wells, T. (2017). Unacylated ghrelin promotes adipogenesis in rodent bone marrow via ghrelin O-acyl transferase and GHS-R1aactivity: Evidence for target cell-induced acylation. Scientific Reports, 7(November 2016), 1-11. http:/ / doi.org/10.1038/srep45541

Hornsby, A. K. E., Redhead, Y. T., Rees, D. J., Ratcliff, M. S. G., Reichenbach, A., Wells, T., ... Davies, J. S. (2016). Short-term calorie restriction enhances adult hippocampal neurogenesis and remote fear memory in a Ghsr-dependent manner. Psychoneuroendocrinology, 63, 198-207. http://doi.org/10.1016/j.psyneuen.2015.09.023

Hornsby, A. K. E., Santos, V. V, Johnston, F., Roberts, L. D., Stark, R., Reichenbach, A., ... Davies, J. S. (2018). Circulating unacylated-ghrelin impairs hippocampal neurogenesis and memory in mice and is altered in human Parkinson\&\#039;s disease dementia. BioRxiv. Retrieved from http://biorxiv.org/content/early/2018/02/05/259333.abstract

Hou, J., Kovacs, M. S., Dhanvantari, S., \& Luyt, L. G. (2018). Development of Candidates for Positron Emission Tomography (PET) Imaging of Ghrelin Receptor in Disease: Design, Synthesis, and Evaluation of Fluorine-Bearing Quinazolinone Derivatives. Journal of Medicinal Chemistry, 61(3), 1261-1275.

http://doi.org/10.1021/acs.jmedchem.7b01754

Howard, A. D., Feighner, S. D., Cully, D. F., Arena, J. P., Liberator, P. A., Rosenblum, C. I., ... Ploeg, L. H. T. Van Der. (1996). A Receptor in Pituitary and Hypothalamus That Functions in Growth Hormone Release. Science, 273(August).

Inui, A. (2004). Ghrelin, appetite, and gastric motility: the emerging role of the stomach as an endocrine organ. The FASEB Journal, 18(3), 439-456. http:/ / doi.org/10.1096/fj.03-0641rev 
Jastrzebska, B. (2013). GPCR: G protein complexes - The fundamental signaling assembly. Amino Acids, 45(6), 1303-1314. http://doi.org/10.1007/s00726-013-1593-y

Jiang, H., Li, L. J., Wang, J., \& Xie, J. X. (2008). Ghrelin antagonizes MPTPinduced neurotoxicity to the dopaminergic neurons in mouse substantia nigra. Experimental Neurology, 212(2), 532-537. http://doi.org/10.1016/j.expneurol.2008.05.006

Johansson, I., Destefanis, S., Aberg, N. D., Aberg, M. a I., Blomgren, K., Zhu, C., ... Isgaard, J. (2008). Proliferative and protective effects of growth hormone secretagogues on adult rat hippocampal progenitor cells. Endocrinology, 149(5), 2191-9. http:// doi.org/10.1210/en.2007-0733

Joo, J. Y., Schaukowitch, K., Farbiak, L., Kilaru, G., \& Kim, T. K. (2015). Stimulus-specific combinatorial functionality of neuronal c-fos enhancers. Nature Neuroscience, 19(1), 75-83. http://doi.org/10.1038/nn.4170

Jungenitz, T., Radic, T., Jedlicka, P., \& Schwarzacher, S. W. (2013). HighFrequency Stimulation Induces Gradual Immediate Early Gene Expression in Maturing Adult-Generated Hippocampal Granule Cells. Cerebral Cortex (New York, N.Y. : 1991). http://doi.org/10.1093/cercor/bht035

Kang, S., Moon, N. R., Kim, D. S., Kim, S. H., \& Park, S. (2015). Central acylated ghrelin improves memory function and hippocampal AMPK activation and partly reverses the impairment of energy and glucose metabolism in rats infused with $\beta$-amyloid. Peptides, 71. http://doi.org/10.1016/j.peptides.2015.07.005

Kaplan, M. S., \& Hinds, J. W. (1977). Neurogenesis in the Adult Rat : Electron Microscopic Analysis of Light Radioautographs. Science, 197(4308), 1092-1094. http://doi.org/10.1126/science.887941 
Katrich, V., Cherezov, V., \& Stevens, R. C. (2013). Structure-Function of the G Protein-Coupled Receptor Superfamily. Journal of Biological Chemistry, 53, 531-56. http:/ / doi.org/10.1146/ ANNUREV-PHARMTOX-032112135923

Kempermann, G., Jessberger, S., Steiner, B., \& Kronenberg, G. (2004).

Milestones of neuronal development in the adult hippocampus. Trends in Neurosciences, 27(8), 447-452.

http:/ / doi.org/10.1016/j.tins.2004.05.013

Kempermann, G., Kuhn, H. G., \& Gage, F. H. (1997). More hippocampal neurons in adult mice living in an enriched environment. Nature. http://doi.org/10.1038/386493a0

Kempermann, G., Song, H., \& Gage, F. H. (2015). Neurogenesis in the Adult Hippocampus. European Journal of Neuroscience, 207, 199-207. http:/ / doi.org/10.1002/hipo.20151

Kenangil, G., \& Ozdilek, B. (2016). Serum Leptin Levels and Cognition in Parkinson's Disease Patients. Noropsikiyatri Arsivi-Archives of Neuropsychiatry, 53(3), 241-244. http:/ / doi.org/10.5152/npa.2016.11319

Kent, B. A., Beynon, A. L., Hornsby, A. K. E., Bekinschtein, P., Bussey, T. J., Davies, J. S., \& Saksida, L. M. (2015). The orexigenic hormone acylghrelin increases adult hippocampal neurogenesis and enhances pattern separation. Psychoneuroendocrinology, 51, 431-439. http://doi.org/10.1016/j.psyneuen.2014.10.015

Kern, A., Mavrikaki, M., Ullrich, C., Albarran-Zeckler, R., Brantley, A., \& Smith, R. G. (2015). Hippocampal Dopamine/DRD1 Signaling Dependent on the Ghrelin Receptor. Cell, 1176-1190. http://doi.org/10.1016/j.cell.2015.10.062

Kim, Y., Kim, S., Kim, C., Sato, T., Kojima, M., \& Park, S. (2015). Ghrelin is required for dietary restriction-induced enhancement of hippocampal 
neurogenesis: lessons from ghrelin knockout mice. Endocrine Journal, 62(3), 269-275. http://doi.org/10.1507/endocrj.

Kitamura, T., Saitoh, Y., Takashima, N., Murayama, A., Niibori, Y., Ageta, H., ... Inokuchi, K. (2009). Adult Neurogenesis Modulates the Hippocampus-Dependent Period of Associative Fear Memory. Cell, 139(4), 814-827. http:// doi.org/10.1016/j.cell.2009.10.020

Klok, M. D., Jakobsdottir, S., \& Drent, M. L. (2007). The role of leptin and ghrelin in the regulation of food intake and body weight in humans: A review. Obesity Reviews, 8(1), 21-34. http:// doi.org/10.1111/j.1467789X.2006.00270.x

Kojima, M., Hamamoto, A., \& Sato, T. (2016). Ghrelin O-acyltransferase (GOAT), a specific enzyme that modifies ghrelin with a medium-chain fatty acid. Journal of Biochemistry, 160(4), 189-194. http://doi.org/10.1093/jb/mvw046

Kojima, M., Hosoda, H., Date, Y., Nakazato, M., Matsuo, H., \& Kangawa, K. (1999). Ghrelin is a growth-hormone-releasing acylated peptide from stomach. Nature, 402(6762), 656-60. http:// doi.org/10.1038/45230

Kojima, M., Hosoda, H., Matsuo, H., \& Kangawa, K. (2001). Ghrelin: Discovery of the natural endogenous ligand for the growth hormone secretagogue receptor. Trends in Endocrinology and Metabolism, 12(3), 118122. http:// doi.org/10.1016/S1043-2760(00)00362-3

Kuhla, A., Lange, S., Holzmann, C., Maass, F., Petersen, J., Vollmar, B., \& Wree, A. (2013). Lifelong caloric restriction increases working memory in mice. PloS One, 8(7), e68778. http://doi.org/10.1371/journal.pone.0068778

Kunath, N., Van Groen, T., Allison, D. B., Kumar, A., Dozier-Sharpe, M., \& Kadish, I. (2015). Ghrelin agonist does not foster insulin resistance but improves cognition in an Alzheimer's disease mouse model. Scientific 
Reports, 5. http:/ / doi.org/10.1038/srep11452

Kuppens, R. J., Diène, G., Bakker, N. E., Molinas, C., Faye, S., Nicolino, M., ... Hokken-Koelega, A. C. S. (2015). Elevated ratio of acylated to unacylated ghrelin in children and young adults with Prader-Willi syndrome.

Endocrine, 50(3), 633-642. http:/ / doi.org/10.1007/s12020-015-0614-x

Lacquaniti, A., Donato, V., Chirico, V., Buemi, A., \& Buemi, M. (2011).

Obestatin: An interesting but controversial gut hormone. Annals of Nutrition and Metabolism, 59(2-4), 193-199.

http://doi.org/10.1159/000334106

Ladran, I., Tran, N., Topol, A., \& Brennand, K. J. (2013). Neural stem and progenitor cells in health and disease. Wiley Interdisciplinary Reviews: Systems Biology and Medicine, 5(6), 701-715.

http://doi.org/https://doi.org/10.1002/wsbm.1239

Leal, S. L., \& Yassa, M. A. (2015). Neurocognitive Aging and the Hippocampus Across Species. Trends in Neuroscience, 38(12), 800-812. http:/ / doi.org/10.1016/j.tins.2015.10.003.Neurocognitive

Lee, J., Duan, W., Long, J. M., Ingram, D. K., \& Mattson, M. P. (2000). Dietary restriction increases the number of newly generated neural cells, and induces BDNF expression, in the dentate gyrus of rats. Journal of Molecular Neuroscience, 15, 99-108. http:/ / doi.org/10.1385/JMN:15:2:99

Lee, J., Seroogy, K. B., \& Mattson, M. P. (2002). Dietary restriction enhances neurotrophin expression and neurogenesis in the hippocampus of adult mice. Journal of Neurochemistry, 80(3), 539-547. http:/ / doi.org/10.1046/j.0022-3042.2001.00747.x

Lemarié, F., Beauchamp, E., Legrand, P., \& Rioux, V. (2016). Revisiting the metabolism and physiological functions of caprylic acid (C8:0) with special focus on ghrelin octanoylation. Biochimie, 120, 40-48. http://doi.org/10.1016/j.biochi.2015.08.002 
Leung, P. K., Chow, K. B. S., Lau, P. N., Chu, K. M., Chan, C. B., Cheng, C. H. K., \& Wise, H. (2007). The truncated ghrelin receptor polypeptide (GHS$\mathrm{R} 1 b)$ acts as a dominant-negative mutant of the ghrelin receptor. Cellular Signalling, 19(5), 1011-1022. http:/ / doi.org/10.1016/j.cellsig.2006.11.011

Li, E., Chung, H., Kim, Y., Kim, D. H., Ryu, J. H., Sato, T., ... Park, S. (2013). Ghrelin directly stimulates adult hippocampal neurogenesis : implications for learning and memory. http:/ / doi.org/10.1507/endocrj.

Li, E., Kim, Y., Kim, S., \& Park, S. (2013). Ghrelin-induced hippocampal neurogenesis and enhancement of cognitive function are mediated independently of GH/IGF-1 axis: lessons from the spontaneous dwarf rats. Endocrine Journal, 60(9), 1065-1075. http:/ / doi.org/10.1507/endocrj.EJ13-0045

Li, E., Kim, Y., Kim, S., Sato, T., Kojima, M., \& Park, S. (2014). Ghrelin stimulates proliferation, migration and differentiation of neural progenitors from the subventricular zone in the adult mice. Experimental Neurology, 252, 75-84. http:/ / doi.org/10.1016/j.expneurol.2013.11.021

Licinio, J., Caglayan, S., Ozata, M., Yildiz, B. O., de Miranda, P. B., O'Kirwan, F., ... Wong, M.-L. (2004). Phenotypic effects of leptin replacement on morbid obesity, diabetes mellitus, hypogonadism, and behavior in leptin-deficient adults. Proceedings of the National Academy of Sciences, 101(13), 4531-4536. http:/ / doi.org/10.1073/pnas.0308767101

Lindqvist, D., Kaufman, E., Brundin, L., Hall, S., Surova, Y., \& Hansson, O. (2012). Non-Motor Symptoms in Patients with Parkinson's Disease Correlations with Inflammatory Cytokines in Serum. PLoS ONE, 7(10). http:/ / doi.org/10.1371/journal.pone.0047387

Lorefält, B., Toss, G., \& Granérus, A.-K. (2009). Weight loss, body fat mass, and leptin in Parkinson's disease. Movement Disorders, 24(6), 885-890. http:/ / doi.org/10.1002/mds.22466 
Lugert, S., Basak, O., Knuckles, P., Haussler, U., Fabel, K., Götz, M., ...

Giachino, C. (2010). Quiescent and active hippocampal neural stem cells with distinct morphologies respond selectively to physiological and pathological stimuli and aging. Cell Stem Cell, 6(5), 445-56.

http://doi.org/10.1016/j.stem.2010.03.017

Lutter, M., Sakata, I., Osborne-lawrence, S., Rovinsky, S. A., Jason, G., Jung, S., ... Zigman, J. M. (2008). Symptoms of Chronic Stress, 11(7), 752-753. http://doi.org/10.1038/nn.2139.The

Mani, B. K., Walker, A. K., Lopez Soto, E. J., Raingo, J., Lee, C. E., Perelló, M., ... Zigman, J. M. (2014). Neuroanatomical characterization of a growth hormone secretagogue receptor-green fluorescent protein reporter mouse. The Journal of Comparative Neurology, 522(16), 3644-66. http:/ / doi.org/10.1002/cne.23627

Mary, S., Fehrentz, J. A., Damian, M., Gaibelet, G., Orcel, H., Verdié, P., ... Banères, J. L. (2013). Heterodimerization with its splice variant blocks the ghrelin receptor 1a in a non-signaling conformation: A study with a purified heterodimer assembled into lipid discs. Journal of Biological Chemistry, 288(34), 24656-24665. http://doi.org/10.1074/jbc.M113.453423

Masumoto, N., Lanyon-Hogg, T., Rodgers, U. R., Konitsiotis, A. D., Magee, A. I., \& Tate, E. W. (2015). Membrane bound O-acyltransferases and their inhibitors. Biochemical Society Transactions, 43(2), 246-252. http:/ / doi.org/10.1042/BST20150018

Maswood, N., Young, J., Tilmont, E., Zhang, Z., Gash, D. M., Gerhardt, G. A., ... Ingram, D. K. (2004). Caloric restriction increases neurotrophic factor levels and attenuates neurochemical and behavioral deficits in a primate model of Parkinson's disease. Proceedings of the National Academy of Sciences, 101(52), 18171-18176. http:/ / doi.org/10.1073/pnas.0405831102 
Mattison, J. A., Colman, R. J., Beasley, T. M., Allison, D. B., Kemnitz, J. W., Roth, G. S., ... Saville, D. J. (2017). Caloric restriction improves health and survival of rhesus monkeys. Nature Communications, 8(May 2016), 14063. http:/ / doi.org/10.1038/ncomms14063

Mattison, J. A., Roth, G. S., Mark Beasley, T., Tilmont, E. M., Handy, A. M., Herbert, R. L., ... De Cabo, R. (2012). Impact of caloric restriction on health and survival in rhesus monkeys from the NIA study. Nature, 489(7415), 318-321. http:/ / doi.org/10.1038/nature11432

Meskenaite, V., Krackow, S., \& Lipp, H.-P. (2016). Age-Dependent Neurogenesis and Neuron Numbers within the Olfactory Bulb and Hippocampus of Homing Pigeons. Frontiers in Behavioral Neuroscience, 10(June), 1-10. http:/ / doi.org/10.3389/fnbeh.2016.00126

Miller, J. A., Guillozet-Bongaarts, A., Gibbons, L. E., Postupna, N., Renz, A., Beller, A. E., ... Lein, E. (2017). Neuropathological and transcriptomic characteristics of the aged brain. ELife, 6, 1-26.

http://doi.org/10.7554/eLife.31126 LK -

http://sfx.metabib.ch/sfx_locater?sid=EMBASE\&issn=2050084X\&id=do i:10.7554\%2FeLife.31126\&atitle=Neuropathological+and+transcriptomic + characteristics + of + the + aged + brain\&stitle $=$ eLife\&title $=$ eLife\&volume $=$ $6 \&$ issue $=\&$ spage $=\&$ epage $=$ \&aulast $=$ Miller\&aufirst $=$ Jeremy + A.\&auinit $=\mathrm{J}$ .A.\&aufull=Miller+J.A.\&coden=\&isbn=\&pages=\&date=2017\&auinit1 $=\mathrm{J} \&$ auinitm $=\mathrm{A}$

Milligan, G. (2007). G protein-coupled receptor dimerisation: Molecular basis and relevance to function. Biochimica et Biophysica Acta-Biomembranes, 1768(4), 825-835. http:/ / doi.org/10.1016/j.bbamem.2006.09.021

Moon, M., Kim, S., Hwang, L., \& Park, S. (2009). Ghrelin regulates hippocampal neurogenesis in adult mice. Endocrine Journal, 56(3), 525531. Retrieved from http:/ / www.ncbi.nlm.nih.gov/pubmed/19506321 
Morgan, A. H., Rees, D. J., Andrews, Z. B., \& Davies, J. S. (2018). Ghrelin mediated neuroprotection - A possible therapy for Parkinson's disease? Neuropharmacology, 1-10. http:/ / doi.org/10.1016/j.neuropharm.2017.12.027

Mu, Y., \& Gage, F. H. (2011). Adult hippocampal neurogenesis and its role in Alzheimer's disease. Molecular Neurodegeneration, 6(1), 85. http:/ / doi.org/10.1186/1750-1326-6-85

Mukherjee, A., Biswas, A., \& Das, S. K. (2016). Gut dysfunction in Parkinson's disease. World Journal of Gastroenterology, 22(25), 5742-5752. http:/ / doi.org/10.3748/wjg.v22.i25.5742

Murtuza, M. I., \& Isokawa, M. (2018). Endogenous ghrelin-O-acyltransferase (GOAT) acylates local ghrelin in the hippocampus. Journal of Neurochemistry, 144(1), 58-67. http:/ / doi.org/10.1111/jnc.14244

Navarro, G., Aguinaga, D., Angelats, E., Medrano, M., Moreno, E., Mallol, J., ... Ferré, S. (2016). A significant role of the truncated ghrelin receptor GHS-R1b in ghrelin-induced signaling in neurons. Journal of Biological Chemistry, 291(25), 13048-13062. http://doi.org/10.1074/jbc.M116.715144

Ngwenya, L. B., Heyworth, N. C., Shwe, Y., Moore, T. L., \& Rosene, D. L. (2015). Age-related changes in dentate gyrus cell numbers, neurogenesis, and associations with cognitive impairments in the rhesus monkey. Frontiers in Systems Neuroscience, 9(July), 1-16. http:/ / doi.org/10.3389/fnsys.2015.00102

Nimchinsky, E. A., Sabatini, B. L., \& Svoboda, K. (2002). Structure and Function of Dendritic Spines. Annual Review of Physiology, 64(1), 313-353. http://doi.org/10.1146/annurev.physiol.64.081501.160008

Nishi, Y., Hiejima, H., Hosoda, H., Kaiya, H., Mori, K., Fukue, Y., ... Kojima, M. (2005). Ingested medium-chain fatty acids are directly utilized for the 
acyl modification of ghrelin. Endocrinology, 146(5), 2255-2264.

http://doi.org/10.1210/en.2004-0695

Nishi, Y., Yoh, J., Hiejima, H., \& Kojima, M. (2011a). Structures and molecular forms of the ghrelin-family peptides. Peptides, 32(11), 2175-82. http://doi.org/10.1016/j.peptides.2011.07.024

Nishi, Y., Yoh, J., Hiejima, H., \& Kojima, M. (2011b). Structures and molecular forms of the ghrelin-family peptides. Peptides, 32(11), 2175-82. http://doi.org/10.1016/j.peptides.2011.07.024

Nokia, M. S., Lensu, S., Ahtiainen, J. P., Johansson, P. P., Koch, L. G., Britton, S. L., \& Kainulainen, H. (2016). Physical exercise increases adult hippocampal neurogenesis in male rats provided it is aerobic and sustained. Journal of Physiology, 594(7), 1855-1873. http://doi.org/10.1113/JP271552

Park, H. R., \& Lee, J. (2011). Neurogenic contributions made by dietary regulation to hippocampal neurogenesis, 1229, 23-28. http://doi.org/10.1111/j.1749-6632.2011.06089.x

Park, J.-H., Glass, Z., Sayed, K., Michurina, T. V, Lazutkin, A., Mineyeva, O., ... Enikolopov, G. (2013). Calorie restriction alleviates the age-related decrease in neural progenitor cell division in the aging brain. The European Journal of Neuroscience, 37(12), 1987-93. http://doi.org/10.1111/ejn.12249

Parkinson's, U. (2018). No Title. Retrieved March 27, 2018, from https://www.parkinsons.org.uk/about-us/media-and-press-office

Paxinos, G., Franklin, K. (2012). Paxinos and Franklin's The Mouse Brain in Stereotaxic Coordinates 4th Edition.

Pfeiffer, R. F. (2003). Gastrointestinal dysfunction in Parkinson's disease. The Lancet Neurology, 2(2), 107-116. http:/ / doi.org/10.1016/S1474- 
4422(03)00307-7

Qin, W., Chachich, M., Lane, M., Roth, G., Bryant, M., de Cabo, R., ... Pasinetti, G. M. (2006). Calorie restriction attenuates Alzheimer's disease type brain amyloidosis in Squirrel monkeys (Saimiri sciureus). Journal of Alzheimer's Disease : JAD, 10(4), 417-422.

Ramalingam, M., \& Kim, S.-J. (2015). The Neuroprotective Role of Insulin Against MPP $\mathrm{p}$-Induced Parkinson $0 \mathrm{~s}$ Disease in Differentiated SHSY5Y Cells. J. Cell. Biochem, 9999(September 2015), 1-10. http://doi.org/10.1002/jcb.25376

Reichenbach, A., Steyn, F. J., Sleeman, M. W., \& Andrews, Z. B. (2012). Ghrelin receptor expression and colocalization with anterior pituitary hormones using a GHSR-GFP mouse line. Endocrinology, 153(11), 54525466. http:/ / doi.org/10.1210/en.2012-1622

Reimer, R. a., Maurer, A. D., Lau, D. C. W., \& Auer, R. N. (2010). Long-term dietary restriction influences plasma ghrelin and GOAT mRNA level in rats. Physiology and Behavior, 99(5), 605-610.

http://doi.org/10.1016/j.physbeh.2010.01.034

Romere, C., Duerrschmid, C., Bournat, J., Constable, P., Jain, M., Xia, F., ... Chopra, A. R. (2016). Asprosin, a Fasting-Induced Glucogenic Protein Hormone. Cell, 165(3), 566-579. http:/ / doi.org/10.1016/j.cell.2016.02.063

Rossi, C., Angelucci, A., Costantin, L., Braschi, C., Mazzantini, M., Babbini, F., ... Caleo, M. (2006). Brain-derived neurotrophic factor (BDNF) is required for the enhancement of hippocampal neurogenesis following environmental enrichment. The European Journal of Neuroscience, 24(7), 1850-6. http:/ / doi.org/10.1111/j.1460-9568.2006.05059.x

Sabatini, B. L., Maravall, M., \& Svoboda, K. (2001). Ca2+ signaling in dendritic spines. Current Opinion in Neurobiology, 11(3), 349-356. http:/ / doi.org/10.1016/S0959-4388(00)00218-X 
Sahay, A., Scobie, K. N., Hill, A. S., O'Carroll, C. M., Kheirbek, M. A., Burghardt, N. S., ... Hen, R. (2011). Increasing adult hippocampal neurogenesis is sufficient to improve pattern separation. Nature, 472(7344), 466-470. http:/ / doi.org/10.1038/nature09817

Sakata, I., Nakano, Y., Osborne-lawrence, S., Rovinsky, S. A., Lee, C. E., Perello, M., ... Zigman, J. M. (2009). Characterization of a Novel Ghrelin Cell Reporter Mouse, 155, 91-98.

http:/ / doi.org/10.1016/j.regpep.2009.04.001.Characterization

Santarelli, L., Saxe, M., Gross, C., Surget, A., Battaglia, F., Dulawa, S., ... Hen, R. (2003). Requirement of hippocampal neurogenesis for the behavioral effects of antidepressants. Science, 301(5634), 805-809. http://doi.org/10.1126/science.1083328

Satou, M., Nishi, Y., Yoh, J., Hattori, Y., \& Sugimoto, H. (2010). Identification and characterization of acyl-protein thioesterase 1/lysophospholipase I as a ghrelin deacylation/lysophospholipid hydrolyzing enzyme in fetal bovine serum and conditioned medium. Endocrinology, 151(10), 47654775. http:/ / doi.org/10.1210/en.2010-0412

Schafer, S. T., Han, J., Pena, M., von Bohlen und Halbach, O., Peters, J., \& Gage, F. H. (2015). The Wnt Adaptor Protein ATP6AP2 Regulates Multiple Stages of Adult Hippocampal Neurogenesis. Journal of Neuroscience, 35(12), 4983-4998. http:/ / doi.org/10.1523/jneurosci.413014.2015

Schellekens, H., De Francesco, P. N., Kandil, D., Theeuwes, W. F., McCarthy, T., Van Oeffelen, W. E. P. A., ... Cryan, J. F. (2015). Ghrelins Orexigenic Effect Is Modulated via a Serotonin 2C Receptor Interaction. ACS Chemical Neuroscience, 6(7), 1186-1197. http://doi.org/10.1021/cn500318q

Shanado, Y., Kometani, M., Uchiyama, H., Koizumi, S., \& Teno, N. (2004). 
Lysophospholipase I identified as a ghrelin deacylation enzyme in rat stomach. Biochemical and Biophysical Research Communications, 325(4), 1487-1494. http://doi.org/10.1016/j.bbrc.2004.10.193

Sherer, T. B. (2011). Biomarkers for Parkinson's Disease, 3(79).

Song, N. N., Jia, Y. F., Zhang, L., Zhang, Q., Huang, Y., Liu, X. Z., ... Ding, Y. Q. (2016). Reducing central serotonin in adulthood promotes hippocampal neurogenesis. Scientific Reports, 6, 1-12. http:// doi.org/10.1038/srep20338

Song, N., Wang, W., Jia, F., Du, X., Xie, A., He, Q., .. Jiang, H. (2017). Assessments of plasma ghrelin levels in the early stages of parkinson's disease. Movement Disorders, 32(10), 1487-1491. http://doi.org/10.1002/mds.27095

Sorrells, S. F., Paredes, M. F., Cebrian-Silla, A., Sandoval, K., Qi, D., Kelley, K. W., ... Alvarez-Buylla, A. (2018). Human hippocampal neurogenesis drops sharply in children to undetectable levels in adults. Nature. http://doi.org/10.1038/nature25975

Spalding, K. L., Bergmann, O., Alkass, K., Bernard, S., Salehpour, M., Huttner, H. B., ... Frisén, J. (2013). Dynamics of hippocampal neurogenesis in adult humans. Cell, 153(6), 1219-1227. http://doi.org/10.1016/j.cell.2013.05.002

Spalding, K. L., Bhardwaj, R. D., Buchholz, B. A., Druid, H., \& Frisén, J. (2005). Retrospective Birth Dating of Cells in Humans. Cell, 122(1), $133-$ 143. http:// doi.org/10.1016/j.cell.2005.04.028

Steiner, B., Klempin, F., Wang, L., Kott, M., Kettenmann, H., \& Kempermann, G. (2006). Type-2 Cells as Link Between Glial and Neuronal Lineage in Adult Hippocampal Neurogenesis, 814(July), 805-814. http://doi.org/10.1002/glia 
Su, X., \& Federoff, H. J. (2014). Immune responses in Parkinson's disease: interplay between central and peripheral immune systems. Biomed Research International, 2014, 275178. http:/ / doi.org/10.1155/2014/275178

Suh, H., Consiglio, A., Ray, J., Sawai, T., D’Amour, K. a, \& Gage, F. H. (2007). In vivo fate analysis reveals the multipotent and self-renewal capacities of Sox2+ neural stem cells in the adult hippocampus. Cell Stem Cell, 1(5), 515-28. http:/ / doi.org/10.1016/j.stem.2007.09.002

Sun, Y., Garcia, J. M., \& Smith, R. G. (2007). Ghrelin and growth hormone secretagogue receptor expression in mice during aging. Endocrinology, 148(3), 1323-1329. http:/ / doi.org/10.1210/en.2006-0782

Sung, H. Y., Park, J. W., \& Kim, J. S. (2014). The frequency and severity of gastrointestinal symptoms in patients with early Parkinson's disease. J Mov Disord, 7(1), 7-12. http:/ / doi.org/10.14802/jmd.14002

Takamura, N., Nakagawa, S., Masuda, T., Boku, S., Kato, A., Song, N., ... Kusumi, I. (2014). The effect of dopamine on adult hippocampal neurogenesis. Progress in Neuro-Psychopharmacology and Biological Psychiatry, 50, 116-124. http:/ / doi.org/10.1016/j.pnpbp.2013.12.011

Tatro, E. T., Risbrough, V., Soontornniyomkij, B., Young, J., ShumakerArmstrong, S., Jeste, D. V., \& Achim, C. L. (2013). Short-term recognition memory correlates with regional CNS expression of microRNA-138 in mice. American Journal of Geriatric Psychiatry, 21(5), 461-473. http://doi.org/10.1016/j.jagp.2012.09.005

Tauber, M., Diene, G., Mimoun, E., Çabal-Berthoumieu, S., Mantoulan, C., Molinas, C., ... Salles, J. P. (2014). Prader-willi syndrome as a model of human hyperphagia. Frontiers of Hormone Research, 42, 93-106. http://doi.org/10.1159/000358317

Taupin, P. (2006). Neurogenesis and the effect of antidepressants. Drug Target Insights, 1(65), 13-17. 
Taylor, M. S., Ruch, T. R., Hsiao, P. Y., Hwang, Y., Zhang, P., Dai, L., ... Cole, P. A. (2013). Architectural organization of the metabolic regulatory enzyme ghrelin O-acyltransferase. Journal of Biological Chemistry, 288(45), 32211-32228. http:/ / doi.org/10.1074/jbc.M113.510313

Thuret, S., Toni, N., Aigner, S., Yeo, G. W., \& Gage, F. H. (2009).

Hippocampus-dependent learning is associated with adult neurogenesis in MRL/MpJ mice. Hippocampus, 19(7), 658-669.

http:/ / doi.org/10.1002/hipo.20550

Unger, M. M., Hattemer, K., Möller, J. C., Schmittinger, K., Mankel, K., Eggert, K., ... Knake, S. (2010). Real-time visualization of altered gastric motility by magnetic resonance imaging in patients with Parkinson's disease. Movement Disorders, 25(5), 623-628.

http://doi.org/10.1002/mds.22841

Unger, M. M., Möller, J. C., Mankel, K., Eggert, K. M., Bohne, K., Bodden, M., ... Oertel, W. H. (2011). Postprandial ghrelin response is reduced in patients with Parkinson's disease and idiopathic REM sleep behaviour disorder: a peripheral biomarker for early Parkinson's disease? Journal of Neurology, 258(6), 982-90. http:// doi.org/10.1007/s00415-010-5864-1

Unger, M. S., Marschallinger, J., Kaindl, J., Höfling, C., Rossner, S., Heneka, M. T., ... Aigner, L. (2016). Early Changes in Hippocampal Neurogenesis in Transgenic Mouse Models for Alzheimer's Disease. Molecular Neurobiology, 53(8), 5796-5806. http:/ / doi.org/10.1007/s12035-016-00189

Urban, D. J., \& Roth, B. L. (2015). DREADDs (Designer Receptors Exclusively Activated by Designer Drugs): Chemogenetic Tools with Therapeutic Utility. Annual Review of Pharmacology and Toxicology, 55(1), 399-417. http:/ / doi.org/10.1146/annurev-pharmtox-010814-124803

van Woerden, G. M., Harris, K. D., Hojjati, M. R., Gustin, R. M., Qiu, S., de 
Avila Freire, R., ... Elgersma, Y. (2007). Rescue of neurological deficits in a mouse model for Angelman syndrome by reduction of aCaMKII inhibitory phosphorylation. Nature Neuroscience, 10(3), 280-282. http:/ / doi.org/10.1038/nn1845

Veyrac, A., Gros, A., Bruel-Jungerman, E., Rochefort, C., Kleine Borgmann, F. B., Jessberger, S., \& Laroche, S. (2013). Zif268/egr1 gene controls the selection, maturation and functional integration of adult hippocampal newborn neurons by learning. Proceedings of the National Academy of Sciences of the United States of America. http://doi.org/10.1073/pnas.1220558110

Watson, G. S., Cholerton, B. A., Reger, M. A., Baker, L. D., Plymate, S. R., Asthana, S., ... Craft, S. (2005). Preserved Cognition in Patients With Early Alzheimer Disease and Amnestic Mild Cognitive Impairment During Treatment With Rosiglitazone. The American Journal of Geriatric Psychiatry, 13(11), 950-958. http:/ / doi.org/10.1097/00019442-20051100000005

Wellman, M. K., Patterson, Z. R., MacKay, H., Darling, J. E., Mani, B. K., Zigman, J. M., ... Abizaid, A. (2015). Novel Regulator of Acylated Ghrelin, CF801, Reduces Weight Gain, Rebound Feeding after a Fast, and Adiposity in Mice. Frontiers in Endocrinology, 6(September), 1-12. http:/ / doi.org/10.3389/fendo.2015.00144

Werner, S., Unsicker, K., \& von Bohlen und Halbach, O. (2011). Fibroblast growth factor-2 deficiency causes defects in adult hippocampal neurogenesis, which are not rescued by exogenous fibroblast growth factor-2. Journal of Neuroscience Research, 89(10), 1605-1617. http://doi.org/10.1002/jnr.22680

Wesnes, K. A., \& Burn, D. J. (2014). Compromised Object Pattern Separation Performance in Parkinson's Disease Suggests Dentate Gyrus Neurogenesis may be Compromised in the Condition. Journal of 
Alzheimer's Disease \& Parkinsonism, 04(01), 5-8.

http://doi.org/10.4172/2161-0460.1000131

Willesen, M. G., Kristensen, P., \& Rømer, J. (1999). Co-localization of growth hormone secretagogue receptor and NPY mRNA in the arcuate nucleus of the rat. Neuroendocrinology, 70(5), 306-316.

http:// doi.org/10.1159/000054491

Willis, A. W., Schootman, M., Kung, N., Evanoff, B. A., Perlmutter, J. S., \& Racette, B. A. (2012). Predictors of survival in patients with Parkinson disease. Archives of Neurology, 69(5), 601-607. http://doi.org/10.1001/archneurol.2011.2370

Witte, A. V., Fobker, M., Gellner, R., Knecht, S., \& Floel, A. (2009). Calorie restriction improves memory in elderly humans. PNAS, 106(14).

Yang, J., Brown, M. S., Liang, G., Grishin, N. V, \& Goldstein, J. L. (2008). Identification of the acyltransferase that octanoylates ghrelin, an appetite-stimulating peptide hormone. Cell, 132(3), 387-96. http://doi.org/10.1016/j.cell.2008.01.017

Yassa, M. A., Mattfeld, A. T., Stark, S. M., \& Stark, C. E. L. (2011). Age-related memory deficits linked to circuit-specific disruptions in the hippocampus. Proceedings of the National Academy of Sciences, 108(21), 8873-8878. http://doi.org/10.1073/pnas.1101567108

Yassa, M. A., \& Stark, C. E. L. (2011). Pattern separation in the hippocampus. Trends in Neurosciences, 34(10), 515-525. http://doi.org/10.1016/j.tins.2011.06.006.Pattern

Yoshimura, S., Takagi, Y., Harada, J., Teramoto, T., Thomas, S. S., Waeber, C., ... Moskowitz, M. a. (2001). FGF-2 regulation of neurogenesis in adult hippocampus after brain injury. Proceedings of the National Academy of Sciences of the United States of America, 98(10), 5874-5879. http://doi.org/10.1073/pnas.101034998 
Zanardini, R., Benussi, L., Fostinelli, S., Saraceno, C., Ciani, M., Borroni, B., ... Ghidoni, R. (2017). Serum C-Peptide, Visfatin, Resistin, and Ghrelin are Altered in Sporadic and GRN-Associated Frontotemporal Lobar Degeneration. Journal of Alzheimer's Disease : JAD. http://doi.org/10.3233/JAD-170747

Zhang, J. V., Jahr, H., Luo, C.-W., Klein, C., Van Kolen, K., Ver Donck, L., ... Hsueh, A. J. W. (2008). Obestatin Induction of Early-Response Gene Expression in Gastrointestinal and Adipose Tissues and the Mediatory Role of G Protein-Coupled Receptor, GPR39. Molecular Endocrinology, 22(6), 1464-1475. http:// doi.org/10.1210/me.2007-0569

Zhang, J. V., Ren, P. G., Avsian-Kretchmer, O., Luo, C. W., Rauch, R., Klein, C., \& Hsueh, A. J. W. (2005). Obestatin, a peptide encoded by the ghrelin gene, opposes ghrelin's effects on food intake. Science, 310(5750), 996999. http:/ / doi.org/10.1126/ science.1117255

Zhang, Y., Fang, F., Goldstein, J. L., Brown, M. S., \& Zhao, T.-J. (2015).

Reduced autophagy in livers of fasted, fat-depleted, ghrelin-deficient mice: reversal by growth hormone. Proceedings of the National Academy of Sciences of the United States of America, 112(4), 1226-31. http://doi.org/10.1073/pnas.1423643112

Zhao, C., Deng, W., \& Gage, F. H. (2008a). Mechanisms and functional implications of adult neurogenesis. Cell, 132(4), 645-60. http://doi.org/10.1016/j.cell.2008.01.033

Zhao, C., Deng, W., \& Gage, F. H. (2008b). Mechanisms and functional implications of adult neurogenesis. Cell, 132(4), 645-60. http://doi.org/10.1016/j.cell.2008.01.033

Ziemssen, T., \& Reichmann, H. (2007). Non-motor dysfunction in Parkinson's disease. Parkinsonism and Related Disorders, 13(6), 323-332. http:/ / doi.org/10.1016/j.parkreldis.2006.12.014 
Zigman, J. M., Nakano, Y., Coppari, R., Balthasar, N., Marcus, J. N., Lee, C. E., ... Elmquist, J. K. (2005). Mice lacking ghrelin receptors resist the development of diet-induced obesity. The Journal of Clinical Investigation, 115(12), 3564-3572. http:/ / doi.org/10.1172/JCI26002 